\title{
Sorting \& Sequencing Flies by Size: \\ Identification of novel TOR regulators and Parameters for Successful Sorting
}

\author{
Katrin Strassburger ${ }^{1}$, Tanja Zöller ${ }^{1}$, Thomas Sandmann ${ }^{2}$, Svenja Leible $^{2}$, \\ Grainne Kerr², Michael Boutros ${ }^{2}$ and Aurelio A. Teleman ${ }^{1, *}$ \\ ${ }^{1}$ German Cancer Research Center (DKFZ), Heidelberg, Germany \\ ${ }^{2}$ Division of Signalling and Functional Genomics, German Cancer Research \\ Center (DKFZ), Heidelberg, Germany
}

*correspondence: a.teleman@dkfz.de

tel:+49 $622142-1620$

fax: $+49622142-1629$

running title: Linking growth phenotypes and genotypes 


\section{Abstract}

As DNA sequencing throughput increases, novel strategies for discovering genes that affect traits of interest become available. One strategy starts with a population of animals and selects individuals over multiple generations for a particular trait. Subsequent whole genome sequencing should identify loci affecting this trait. We apply this strategy by sorting flies for wing length over 18 generations, obtaining two populations that differ in wing length by $20 \%$. Flies with longer wings had increased overall body sizes and elevated TOR activity, suggesting that genetic variation targets TOR signaling to influence body size. High-throughput sequencing of big and small flies identified thousands of single nucleotide polymorphisms that differed between the two populations, leading us to identify five novel regulators of TOR signaling. Surprisingly, stochastic simulations of the process show that large fractions of the genetic differences between the big and small flies are probably biological false positives, selected by chance by random drift. We employ these computer simulations to identify experimental setup parameters to improve the signal-to-noise ratio for successfully running sort-and-sequence experiments - a resource which will hopefully be useful for the community. 


\section{Introduction}

Forward-genetics has successfully been employed to discover genes affecting a particular biological process of interest in a variety of model organisms. Typically, as few genes as possible are perturbed per animal, and then large numbers of individuals are screened for the trait of interest. This couples a genomic perturbation to the phenotypic output. This approach is successful at identifying genes with strong roles in the biological process of interest, but is less well suited towards identifying genes with redundant roles, or for studying complex traits that are modulated via interactions amongst large numbers of genes. The reduced cost of whole genome sequencing has enabled a novel approach leveraging the broad genetic variability present in nature, rather than experimentally-induced mutations, by combining laboratory-based evolution with complete-genome sequencing. Conceptually, a selective pressure is applied to the population to enrich for variants affecting the selected trait. One implementation of this approach is to start with one population of animals and to select for a trait of interest in the laboratory over several generations to generate two differing end-populations, followed by whole-genome sequencing to identify the genetic differences between the two populations ("sort-and-sequence"). Other implementations can also be envisaged - such as performing a single round of selection followed by sequencing - which can also be applied to a human population in the form of a genome-wide association study. Unlike the forward genetics approach, where each experimental animal has only a few mutations with strong phenotypic consequences, in the 'sort-and-sequence' approach each individual animal in a population may have many genetic differences 
compared to the other animals. Therefore, one needs to sequence a large number of individuals to find correlations and to pinpoint relevant genetic loci.

Animal size is a complex trait, affected by many genetic loci (GockEL et al. 2002; Lango Allen et al. 2010; Lettre 2011; Perola 2011). Despite strong interest in uncovering the molecular mechanisms by which developing tissues and organs can measure their size and regulate their growth, we do not yet have a good understanding of these processes. Forward and reverse genetic approaches, in particular in Drosophila, have identified genes affecting organ size (Stocker and Hafen 2000; Johnston and Gallant 2002; MiRTH and RIDDIFORD 2007; SHINGLETON 2010). In particular, components of two major signaling pathways have been found: the insulin/insulin-like growth factor signaling (IIS) pathway and the Hippo/Yorkie pathway (OLDHAM and HAFEN 2003; PAN 2007; TUMANENG et al. 2012). The IIS pathway consists of a number of kinases including PI3K, Akt, PDK1 and TOR, which become activated in response to hormonal signaling and environmental cues. These kinases in turn activate a plethora of anabolic processes and repress catabolic processes in the cell (LOEWITH and HALL 2011; LAPLANTE and SABATINI 2012). One interpretation is that the IIS pathway coordinately scales the size of all tissues in the organism in response to nutrient availability, thereby generating well-proportioned animals of different sizes based on environmental conditions. A second signaling pathway, the Hippo/Yorkie pathway, also has the capacity to powerfully regulate tissue growth. This pathway consists of several kinases including Hippo and Warts, which act in concert to block activation of a transcriptional co-activator Yorkie. When 
active, Yorkie potently drives cell growth and proliferation by regulating gene transcription (PAN 2007; GeneVet and TAPON 2011; StAley and IRVINE 2012; TUMANENG et al. 2012). The upstream regulatory inputs into the Hippo/Yorkie pathway have been a subject of much recent interest (GRUSCHE et al. 2010; YU and GUAN 2013). This pathway promotes tissue growth in response to wounding (YU and GUAN 2013). Whether Yorkie activity also drops at the end of normal animal development to control normal body size is not yet fully understood.

As a test-of-concept for the "sort-and-sequence" approach, we started with one population of Drosophila melanogaster and selected for wing length over 18 generations, yielding a population of 'big' and 'small' flies. Molecular analysis revealed that the 'big' and 'small' flies differed in their level of activation of the TOR pathway, but not in activation of the Hippo/Yorkie pathway, suggesting that the selective pressure mainly manipulated the IIS pathway to evolve body size. By whole-genome sequencing we identified almost 7000 Single Nucleotide Polymorphisms (SNPs) whose frequency was significantly different between these two populations, including five novel regulators of TOR. Based on the surprising result that only few of the perturbed genes had an effect on TOR activity, we asked whether the experimental setup can be optimized to improve the frequency of biologically relevant hits. We generated an in silico simulation of the sort-and-sequence approach which identifies parameter combinations that significantly reduce the false-positive rate. This will hopefully be a useful resource for the community to successfully set up sort-and-sequence types of experiments. 


\section{Results}

Sorting flies for wing length successfully generates populations of differing wing length within few generations

To identify genes affecting tissue size, we sorted flies according to wing length (Figure 1A). To obtain a starting population with sufficient genetic variability, we intercrossed two inbred fly strains, $w^{1118}$ and Oregon-R, for 3 generations. We then grew these animals in density-controlled conditions, quantified the wing length of 150 male and 150 female flies, and divided the population in half - flies with 'big' wings (length greater than the population mean) and flies with 'small' wings (smaller than the population mean). We repeated this process for 18 generations, consistently retaining either the larger $50 \%$ of the population or the smaller $50 \%$ of the population. As a consequence of this selection, average wing length in the two populations consistently diverged (Figure 1B) starting from the first generation. The difference in the average wing length between the two populations increased almost linearly, yielding two populations with an average wing length difference of $20 \%$ (Figure 1B'). Interestingly, compared to the distribution of wing-lengths in the starting population (Figure 1C, black trace), the distribution of wing-lengths in the final 'big' population displayed a smaller standard deviation (Figure 1C, green trace) whereas the distribution of wing-length in the final 'small' population was increased (Figure 1C, red trace). This lack of homogeneity within the small population could potentially occur if some SNPs causing reduced wing length represented a competitive disadvantage, e.g. display lethality when homozygous, not allowing them to become 'fixed' in the 
population. Flies with wing lengths either smaller or larger than the extremes observed in the initial population were easily observed after a few rounds of sorting (Figure 1C), indicating that the wing lengths in the apparently homogeneous starting population actually reflect the activities of multiple variants counteracting each other.

Selection for wing length caused concomitant selection for total body size Next, we examined whether modulation of wing size was correlated with a change in overall body size, e.g. if these traits can be selected for independently. Flies from the 'small' population displayed reduced wing size (Figure 2A), lower total body weight (Figure 2B), and reduced overall body size (Figure 2C) compared to flies from the 'big' population. The difference in body size was already visible at the end of the larval phases when animals formed pupae (Figure 2D). These results suggest that in our starting population, genetic variants affecting total body size were more common than genetic variants specifically affecting wing length. In contrast, embryo size was not significantly different for big and small flies (Figure 2E), indicating that different processes control adult size and egg size.

'Big' and 'small' flies were selected for differing levels of TOR activation during growth stages

We next aimed to characterize the underlying molecular changes leading to the different body sizes of the two populations. One way tissues size can be modulated is through changes in cell size. We quantified the cell size in wings of 'big' and 'small' flies and found that cells from the 'small' flies were roughly 
$10 \%$ smaller than cells from the 'big' flies (Figure $3 A$ ). Since the wing area of 'small' flies was $25 \%$ smaller than that of 'big' flies (Figure 2A), this indicates that the wings of 'small' flies contained both smaller cells and fewer cells. Signaling via TOR complex 1 (TOR-C1) is known to regulate tissue size in part via altered cell size (STOCKER and HAFEN 2000; ZHANG et al. 2000), raising the possibility that TOR-C1 signaling might be altered in 'small' versus 'big' flies. Since TOR-C1 also regulates organismal metabolism, we asked whether 'big' and 'small' flies had altered metabolic profiles. Indeed, 'small' flies had disproportionately lower levels of triglycerides than 'big' flies, both when normalized to total body protein or body weight (Figure 3B and data not shown). A priori, it was unexpected that selecting flies based on wing length should also lead to two populations with strikingly different triglyceride levels. The fact that TOR-C1 regulates both tissue size and organismal metabolism provides a possible underlying mechanistic link to this coupling.

To assay TOR-C1 signaling directly, we tested the level of phosphorylation of the canonical TOR-C1 targets S6K and 4EBP in extracts of 'big' and 'small' larvae and adults (Figure $3 \mathrm{C}$ ). Phosphorylation of S6K and 4E-BP were increased in lysates from 'big' larvae compared to 'small' larvae (Figure 3C), indicating increased TOR-C1 activation in the 'big' larvae. Interestingly, TORC1 activity was not as strongly affected in 'big' versus 'small' adults (Figure $3 C)$, indicating that the genetic differences selected in the two populations specifically affected TOR-C1 activity during the larval phases of development when organismal growth takes place. In contrast to TOR-C1 activity, phosphorylation of Akt on Ser505, a readout for TOR-C2 activity, was not 
increased in 'big' versus 'small' larvae (Figure 3C). Furthermore, activation of Yorkie, assessed via a standard inhibitory phosphorylation on Ser168 which reflects activation of the Hippo pathway, was similar in big and small animals (Figure $3 C$ ). In sum, these data suggest that the genetic variation present in our flies affected body size at least in part via regulation of TOR-C1 activity during juvenile phases, but not through modulation of the Hippo/Yorkie pathway.

Whole-genome sequencing reveals many SNPs differentially enriched in the ‘big' versus 'small’ populations

To identify genetic differences underpinning the altered body size in the 'big' versus 'small' populations, we performed whole genome sequencing on genomic DNA extracted from the two populations. We sequenced genomic DNA pooled from either 50 females (i.e. 100 alleles) from the 'big' population or 50 females from the 'small' population at 30-fold coverage. The number of chromosomes assayed was chosen to be higher than the average sequencing depth, so that the data effectively samples the population (i.e. reads obtained for a given genomic position are probably from different chromosomes within the population). We then asked which SNPs were differentially represented in the two populations. We quantified two parameters for each position in the genome: 1) a measure for the difference in nucleotide frequency between the two populations and 2) a statistical score for the significance of this difference (see Materials \& Methods for SNP calling). Selecting only SNPs that were completely polarized in the two populations and applying a stringent $p$-value cutoff identified 6984 SNPs with differential frequencies between the two 
populations (Supplemental Table 1, and Experimental Procedures for details), 368 of which led to changes in the amino acid sequences of 279 different genes (Supplemental Figure 2). None of the 279 genes was a known component of the IIS pathway, suggesting that some of the SNPs might affect novel regulators of IIS/TOR signaling.

To assess if these candidate genes can affect TOR-C1 activity, we performed RNAi in S2 cells on all 279 genes and assessed phosphorylation of S6K at Thr398. Knockdown of five candidate genes reduced TOR-C1 activity (Figure 4), validating a functional role in TOR-C1 regulation for these loci. Interestingly, mutation of one of these genes, mip130, yields viable adults with small body sizes, confirming that this gene can affect body size (BEALL et al. 2004).

\section{Generation of an in silico simulation of fly sorting}

Of the 279 genes whose protein coding was differentially affected by SNPs in the big and small populations, five affected TOR-C1 in S2 cells (Figure 4), representing roughly a $2 \%$ confirmation rate. We wondered whether the remaining $98 \%$ might represent false-positives, or true hits that affect different aspects of size determination that were not assayed using our TOR readout in S2 cells. To assess if and how the specificity for the experiment could be improved, we modeled the selection process using a stochastic Monte Carlolike simulation of the 'sort-and-sequence' process (see Supplemental Material for computer code in the $\mathrm{C}$ programming language). Briefly, the simulation randomly generates SNPs throughout the genome (Figure $5 \mathrm{~A}$ ), selects a 
subset of them as SNPs that affect the trait of interest (in this case body size) in a dominant or recessive fashion, generates a random initial population of flies with random genotype, and then executes the phenotyping, sorting, and breeding over multiple generations, allowing for meiotic cross-overs in the female germline. Finally, the simulation records the genetic makeup of the final population thereby identifying the SNPs that were differentially represented in the two populations. (See Experimental Procedures for details). The model was constructed with a large number of parameters that can be adjusted to fit what we know about fly genetics and to test different experimental setups. These include the number of SNPs present genomewide, how many of these affect animal size, the number of meiotic crossovers per chromosome in the female germline, the number of flies selected in each generation, and the number of generations the simulation is allowed to proceed. Importantly, as the identity of the simulated SNPs affecting size are specified by the simulation, their frequency can be monitored during the selection process and compared to SNPs that are differentially enriched simply by chance.

We first modeled the experiment by choosing parameters matching the original experimental setup, e.g. sorting 300 flies (150 males and 150 females) per population per generation for 18 generations. We allowed 7 meiotic cross-overs genome-wide per generation in the female germline (MILLER et al. 2012). From sequencing our 'big' versus 'small' flies and by comparing them to a reference genome, we estimated that our initial population, generated by combining two inbred laboratory stocks, contained 
roughly 175,000 SNPs. We estimated that a standard signaling pathway such as the IIS pathway has roughly 30 components, that each component might have two SNPs (as observed in our sequencing), and that 5 different pathways, both signaling and metabolic, might be affecting size, yielding 300 size-affecting SNPs. With these parameters, the model predicted two populations of flies that diverged in average size in a manner similar to what we observed experimentally (Figure 5B). We then asked how many SNPs were differentially selected in the two populations using a statistical measure that was Bonferroni corrected for multiple hypothesis testing. A p-score cut-off of $10^{-50}$ yielded roughly 15,000 differential SNPs whereas a p-score cut-off of $10^{-100}$ yielded 2,600 SNPs that were differentially represented in the two populations, on the same order of magnitude as what we observed experimentally. Surprisingly, at these two cut-offs, $>97 \%$ of the differentially enriched SNPs did not affect the selected trait (Figure 5C). This means that even though these SNPs were truly differentially selected in the two populations, they were not selected due to their size effects, but through genetic drift.

We examined whether applying an even more stringent statistical cut-off would decrease the false positive rate, however increasing it to the maximal possible score of $10^{-250}$, which only identifies on average 2 of the 300 sizeaffecting SNPs, still had a false positive rate of $81 \%$ (Figure $5 \mathrm{C}$ ). Alternatively, we selected SNPs based on an 'enrichment score' cut-off, which describes how different the allele frequencies are in the two populations ( 0 if it is the same in the two populations and 1 if the SNP has become fully polarized in 
the two populations). Using an enrichment cut-off improved the outcome slightly however a stringent cut-off of 0.8 which identified on average 7 of the 300 size-affecting SNPs still had a false-positive rate of $74 \%$ (Figure 5D). In sum, the results from the simulation suggest that many of the SNPs that were differentially selected in the 'big' and 'small' fly populations from our experimental sorting might be unrelated to animal size determination, raising the question how the experimental setup can be improved to increase the true hit rate.

\section{Identification of parameters for optimizing a sort-and-sequence approach}

Next we varied the model parameters to identify experimental conditions with a low false-positive rate. The analysis above suggested that the false positive rate depends strongly on the relative selection pressure on the size-SNPs versus random drift of the neutral SNPs. Selection pressure on the size-SNPs depends on how many SNPs affect size genome-wide. In the extreme example where only one SNP regulates a phenotype, that SNP will immediately become fixed within a few generations. Instead, when many SNPs affect a phenotype, the selection pressure on any one individual SNP is low. For this reason, we varied the number of SNPs affecting size from 20 to 300 (Figure 6A). This parameter will depend on the phenotype being studied, with simple traits regulated by few SNPs and complex phenotypes regulated by many. The random drift in the frequencies of the neutral SNPs is inversely correlated with the number of flies sorted at every generation. Therefore, we varied the number of selected flies per population from 200 to 1600 flies (Figure 6A). For simple phenotypes affected by only 20 SNPs genome-wide, 
selecting 400 flies per population per generation (i.e. 800 flies total) is enough to give a threshold with a false positive rate below $5 \%$ and a discovery rate of $100 \%$ (i.e. all 20 SNPs). In contrast, for complex phenotypes affected by 300 SNPs genome-wide, at least 1600 flies per population (3200 total) need to be sorted to yield a region where the false positive rate is below $5 \%$ yet the discovery rate is non-zero (e.g. 0.5 enrichment threshold gives a $3 \%$ falsepositive rate and a $24 \%$ discovery rate, yielding 70 trait-related SNPs).

Another parameter that can be varied is the genetic complexity of the starting population: how many SNPs are present in the starting flies. This can be done by selecting in- or out-bred animals as a starting point. Although this does not change the rate of drift of any one individual SNP, it reduces the total number of neutral SNPs that have a chance to be enriched by the end of the experiment, and hence the false-positive rate. As shown in Figure 6B for simulations with 100 SNPs affecting the trait of interest and 200 animals sorted per population per generation, reducing the number of SNPs genomewide to 80,000 significantly improves the false-positive rate, whereas increasing the SNPs to 500,000 genome-wide renders the experimental results unusable. Recent estimates from the D. melanogaster reference panel identified over 4.5 million SNPs in their populations (MACKAY et al. 2012), indicating that 500,000 SNPs can easily occur in one starting population.

Lastly, we asked whether performing a sort-and-sequence experiment in biological triplicates, starting with the same initial population of flies, can improve the false-positive rate by looking at only the SNPs that overlap in all 
three replicates. One could imagine that this would remove all the 'noise'. However, as shown in Figure 6C for simulations with 300 trait-affecting SNPs, this only significantly improves the outcome under some conditions. For the simulations performed with 200 flies being sorted (top row Figure 6C), repeating the experiment in triplicate still yields false-positive rates that are at best $60 \%$. In contrast, if the number of flies sorted is increased to 300 , looking only at the overlap of three biological replicates does reduce the false-positive rate (bottom row, Figure $6 \mathrm{C}$ ). That said, this type of setup does not perform better than simply sorting 900 flies per generation (compare Figure 6A 300 SNPs and 800 flies with Figure 6C lower right panel). In sum, these stochastic simulations provide a panel of starting parameters that are necessary for performing sort-and-sequence experiments with improved false-positive rates.

\section{Discussion}

We aimed in this study to employ a new screening strategy for uncovering genetic loci affecting animal size in Drosophila, based on the selection of flies for wing length over multiple generations followed by whole-genome sequencing. Interestingly, the average size of flies in the population could easily be shifted both towards increased size and reduced size, indicating that many naturally occurring genetic variants affect animal size. This observation implies that although the observed size of flies within one population may appear homogeneous, it actually reflects the balancing action of many counteracting SNPs and large genetic diversity within the population. We find that one mechanism by which these naturally occurring SNPs affect animal 
size is via regulation of TOR activity. Astoundingly, the fly sorting was able to select for very specific traits, because TOR activity was increased in big flies compared to small flies specifically during larval stages of development when animal growth is taking place. Furthermore, we found little evidence for modulation of Yorkie activity in big versus small flies, suggesting that modulation of TOR activity might be a more common mechanism by which animal size can vary in nature, compared to modulation of Yorkie activity.

We performed whole-genome sequencing of the big and small fly populations and identified a very large number of SNPs that were differentially enriched in the two populations - circa 7000 using stringent cut-offs - including nonsynonymous changes in the coding sequence of 279 genes. By screening these for effects on TOR-C1 signaling in cell culture, we identified five novel regulators of TOR-C1 which will likely be interesting genes to study in the future. For the remaining 274 genes, a very large number of assays would need to be performed to test if they are size regulators. They could be affecting a number of different signaling pathways and metabolic networks, in a number of different tissues in the animal. Therefore it is difficult to formally exclude them as size regulators. For this reason, we turned to a stochastic computer simulation of the process to suggest how many of them are likely to be true positives. This work revealed that the majority of these differentially selected SNPs can be expected to be unrelated to size regulation, selected by chance due to random drift within the population. This is an important insight, especially since it is difficult to exclude these SNPs experimentally, and it is tempting to believe they are all true. 
An important output of the simulations is an understanding of what experimental setup conditions are necessary for such an approach. We hope this information will constitute a useful resource for the community. One parameter that is clearly important is the number of organisms sorted at each generation. At least for a genome size of Drosophila, a minimum of 800 flies per population per generation is necessary (Figure 6), meaning that novel high throughput methods for selecting flies will be necessary. We sorted 600 flies at each generation based on wing length (150 flies of each sex for each big and small population), which was roughly the maximum of what was feasible by hand, indicating that automated size determination and sorting will be required to improve this setup for the future. An additional parameter that can be manipulated is the complexity of the starting population. Reducing the number of SNPs in the starting population increases the chances of obtaining meaningful data at the end. This can be done, for instance, by some inbreeding, as long as enough phenotypic variation remains for subsequent selection. Lastly, performing the experiment in biological replicates and looking at the data that overlaps does not seem a superior strategy than simply increasing the numbers of animals sorted at each generation.

Although these simulations were done for a particular sort-and-sequence setup - sorting over multiple generations for a given trait - similar logic will likely apply to other studies. For instance, cancer develops through clonal expansion of cells which accumulate mutations over time, some of which influence cancer development, and some of which do not. Sequencing the 
end population of cells does a good job at identifying the differences compared to matched non-cancer tissue, however identifying which of these mutations is biologically meaningful is a problem well known to members of the field. Likewise genome-wide association studies (GWAS) are similar to the setup employed in this study, except that only one round of selection is performed and individuals are sequenced separately. (Since in our case we selected SNPs that were entirely polarized in the two populations, sequencing the population or sequencing individuals separately yields the same result.) Also for GWAS studies, due to similar types of issues, statistical thresholds are placed very stringently so that only the SNPs with the largest magnitude effects can be identified. Perhaps, similar types of simulations as the ones described here might help, although the parameters for the simulations, such as how many cells are being selected that lead to a tumor, and what the mutation rate is, are difficult to obtain. We hope the data presented here will help to set-up sort-and-sequence strategies to successfully exploit this promising technology in the future.

\section{Experimental Procedures}

Fly stocks and antibodies

$\mathrm{w}^{1118}$ and Oregon-R were obtained from the Bloomington stock collection. Anti phospho-S6K(Thr398), phospho-4E-BP, phospho-Akt, and total-Akt were from Cell Signaling. Anti total-S6K (HAHN et al. 2010), total-4EBP (TELEMAN et al. 2005), phospho-Yki and total Yki (DoNG et al. 2007), anti-tubulin (Developmental Studies Hybridoma Bank). 


\section{Physiological measurements}

All animals were grown under controlled density conditions of 60 animals per vial. Size and lipid quantifications were performed as previously described (TELEMAN 2010). Adult wing cell size was quantified by measuring area per trichome.

\section{Cell culture and dsRNA treatment}

S2 cells were cultured in Express-Five serum-free medium (Invitrogen). After 5 days of treatment with $12 \mu \mathrm{g} / \mathrm{mL}$ dsRNA, immunoblotting was performed on the cell lysates to detect phospho-S6K(Thr398) and total-S6K using the antibodies described above.

\section{Sequencing, bioinformatics and data analysis}

Libraries were generated using the Applied Biosystems SOLiD 3 system standard fragment protocol and sequenced on the SOLiD 3 system. Base calling and quality scoring were done during SOLiD primary analysis. csfasta and qual files of sequencing reads were retrieved from the sequencing machine. Reads were mapped to the Drosophila melanogaster genome using the MapReads algorithm, part of the Corona Lite Pipeline supplied by Applied Biosystems (CoronaLite_v4.0 release 2.0). The genome multi-fasta file was downloaded from the UCSC genome browser (April 2006 Assembly [dm3, BDGP Release 5]). The reference genomes were validated and double encoded as part of the corona lite SNP calling pipeline. The genome coverage for each sample was calculated and a consensus sequence determined using 
Corona Lite pipeline (McKeRnAN et al. 2009). Averaging across all samples, 51 million reads were sequenced per sample, with 14 million reads mapping to unique starting positions, totaling $63 \%$ of the genome coverage, with an accuracy of $99 \%$. Only "valid adjacent color changes" where considered for SNP analysis.

For SNP calling, two measures were calculated for each chromosomal position in the 'big' and 'small' fly populations: 1) the difference in nucleotide frequency between the two populations quantified as the geometric distance in 4D space: $\left(\% \mathrm{~A}_{\text {big }}-\% \mathrm{~A}_{\text {small }}\right)^{2}+\left(\% \mathrm{C}_{\text {big }}-\% \mathrm{C}_{\text {small }}\right)^{2}+\left(\% \mathrm{G}_{\text {big }}-\% \mathrm{G}_{\text {small }}\right)^{2}+\left(\% \mathrm{~T}_{\text {big }}-\right.$ $\left.\% \mathrm{~T}_{\text {small }}\right)^{2}$. 2) A p-score for the significance of this difference calculated using a binomial distribution probability, bonferroni-adjusted for multiple testing. First, the nucleotide frequencies observed in the 'small' fly population were used to derive an underlying frequency distribution that was used to calculate the probability of sampling the nucleotide frequencies observed in the 'big' population using a binomial distribution. To be conservative and to correct for low sequence coverage in the 'small' population, the underlying nucleotide distribution at any one position was initially assumed to be $25 \% \mathrm{~A} / 25 \% \mathrm{C} / 25 \% \mathrm{G} / 25 \% \mathrm{~T}$, with each sequence read skewing this distribution: $\% A_{\text {underlying distribution }}=\left(0.25+A_{\text {small }}\right) /\left(1+A_{\text {small }}+C_{\text {small }}+G_{\text {small }}+T_{\text {small }}\right)$ where $A_{\text {small }}$ is the number of times A was read at that position in the small population, and equivalently for the other nucleotides. Hence, high sequence coverage leads to an underlying distribution almost equal to the one observed by sequencing, whereas low sequence coverage is skewed towards equal representation of the four nucleotides, thereby reducing the significance of observing any 
particular nucleotide distribution in the big flies. Secondly, the equivalent probability of observing the 'small' frequencies based on an underlying 'big' distribution was also calculated. Third, to be conservative, the less-significant of the two p-scores was retained, and Bonferroni-corrected for the number of nucleotides in the genome. For this study, a stringent cut-off of difference=2 and $\mathrm{p}$-score $=0$ was used.

\section{Stochastic Simulation}

The full simulation code in the $\mathrm{C}$ programming language is available in Supplemental Material. The in silico simulation starts with an initial population of flies (Figure 5A). Each fly contains 3 chromosomes as in Drosophila melanogaster. (Chromosome 4 is very small and was therefore ignored for simplicity). Along the chromosomes, SNP positions were randomly specified. Each SNP position could be filled with one of two possible nucleotides (for instance, 'A' or ' $C$ '), to reflect that most SNPs in a population are binary (i.e. one usually finds 2 of the 4 possible nucleotides at that position), and for the sake of simplicity. A random subset of these SNPs was chosen to quantitatively affect animal size (or any quantitative trait of interest) whereas the majority of SNP positions were considered neutral. The SNP positions affecting size were randomly chosen to be either recessive (i.e. they only affect animal size when homozygous in the animal) or dominant (in which case each of the two alleles contributes additively to the quantitative trait, so that heterozygous animals experience half the effect of homozygous animals for that position). The quantitative strength of the contribution towards total animal size for each 'size SNP' was chosen randomly within a 10-fold 
dynamic range, reflecting that not all SNPs are expected to have equally strong effects on animal size. Once this genetic landscape was established, each starting fly within the population was given a random genotype. The size of each animal was calculated, and the larger $50 \%$ or smaller $50 \%$ of the populations retained for breeding to create the next generation. The genotype of each individual in the next generation was compiled by randomly choosing one father and one mother from the breeding animals. Meiotic cross-overs between chromosomes were allowed to occur at random positions within the female germline but not the male germline. The size of each resulting animal was calculated and the selection process was reiterated for a given number of generations. Finally, the genetic makeup of the final population was recorded, including the allele frequency for each SNP in the 'big' and 'small' populations, thereby identifying the SNPs that were differentially represented in the two populations. The simulation allows for all of the above-mentioned parameters to be varied.

\section{Acknowledgements}

This work was supported by a Helmholtz Young Investigator Grant and an ERC Starting Grant to A.A.T. and an ERC Advanced Grant to MB. Antibodies were obtained from the Developmental Studies Hybridoma Bank developed under the auspices of the NICHD and maintained by The University of lowa.

\section{References}


Beall, E. L., M. Bell, D. Georlette and M. R. Botchan, 2004 Dm-myb mutant lethality in Drosophila is dependent upon mip130: positive and negative regulation of DNA replication. Genes Dev 18: 1667-1680.

Dong, J., G. Feldmann, J. Huang, S. Wu, N. Zhang et al., 2007 Elucidation of a universal size-control mechanism in Drosophila and mammals. Cell 130: 1120-1133.

Genevet, A., and N. Tapon, 2011 The Hippo pathway and apico-basal cell polarity. Biochem J 436: 213-224.

Gockel, J., S. J. Robinson, W. J. Kennington, D. B. Goldstein and L. Partridge, 2002 Quantitative genetic analysis of natural variation in body size in Drosophila melanogaster. Heredity (Edinb) 89: 145-153.

Grusche, F. A., H. E. Richardson and K. F. Harvey, 2010 Upstream regulation of the hippo size control pathway. Curr Biol 20: R574-582.

Hahn, K., M. Miranda, V. A. Francis, J. Vendrell, A. Zorzano et al., 2010 PP2A regulatory subunit PP2A-B' counteracts S6K phosphorylation. Cell Metab 11: 438-444.

Johnston, L. A., and P. Gallant, 2002 Control of growth and organ size in Drosophila. Bioessays 24: 54-64.

Lango Allen, H., K. Estrada, G. Lettre, S. I. Berndt, M. N. Weedon et al., 2010 Hundreds of variants clustered in genomic loci and biological pathways affect human height. Nature 467: 832-838.

Laplante, M., and D. M. Sabatini, 2012 mTOR signaling in growth control and disease. Cell 149: 274-293.

Lettre, G., 2011 Recent progress in the study of the genetics of height. Hum Genet 129: 465-472. 
Loewith, R., and M. N. Hall, 2011 Target of rapamycin (TOR) in nutrient signaling and growth control. Genetics 189: 1177-1201.

Mackay, T. F., S. Richards, E. A. Stone, A. Barbadilla, J. F. Ayroles et al., 2012 The Drosophila melanogaster Genetic Reference Panel. Nature 482: 173-178.

McKernan, K. J., H. E. Peckham, G. L. Costa, S. F. McLaughlin, Y. Fu et al., 2009 Sequence and structural variation in a human genome uncovered by short-read, massively parallel ligation sequencing using two-base encoding. Genome Res 19: 1527-1541.

Miller, D. E., S. Takeo, K. Nandanan, A. Paulson, M. M. Gogol et al., 2012 A Whole-Chromosome Analysis of Meiotic Recombination in Drosophila melanogaster. G3 (Bethesda) 2: 249-260.

Mirth, C. K., and L. M. Riddiford, 2007 Size assessment and growth control: how adult size is determined in insects. Bioessays 29: 344-355.

Oldham, S., and E. Hafen, 2003 Insulin/IGF and target of rapamycin signaling: a TOR de force in growth control. Trends Cell Biol 13: 79-85.

Pan, D., 2007 Hippo signaling in organ size control. Genes Dev 21: 886-897.

Perola, M., 2011 Genetics of human stature: Lessons from genome-wide association studies. Horm Res Paediatr 76 Suppl 3: 10-11.

Shingleton, A. W., 2010 The regulation of organ size in Drosophila: Physiology, plasticity, patterning and physical force. Organogenesis 6: 76-87.

Staley, B. K., and K. D. Irvine, 2012 Hippo signaling in Drosophila: recent advances and insights. Dev Dyn 241: 3-15. 
Stocker, H., and E. Hafen, 2000 Genetic control of cell size. Curr Opin Genet Dev 10: 529-535.

Teleman, A. A., 2010 miR-200 de-FOGs insulin signaling. Cell Metab 11: 8-9.

Teleman, A. A., Y. W. Chen and S. M. Cohen, 2005 4E-BP functions as a metabolic brake used under stress conditions but not during normal growth. Genes Dev 19: 1844-1848.

Tumaneng, K., R. C. Russell and K. L. Guan, 2012 Organ size control by Hippo and TOR pathways. Curr Biol 22: R368-379.

Yu, F. X., and K. L. Guan, 2013 The Hippo pathway: regulators and regulations. Genes Dev 27: 355-371.

Zhang, H., J. P. Stallock, J. C. Ng, C. Reinhard and T. P. Neufeld, 2000 Regulation of cellular growth by the Drosophila target of rapamycin dTOR. Genes Dev 14: 2712-2724.

(Figure Legends start on next page) 


\section{Figure Legends}

Figure 1: Sorting flies for wing length over 18 generations yields $20 \%$ difference in size

(A) Schematic representation of the sorting approach to select flies for differing wing sizes. From a single population of flies, the upper and lower $50 \%$ of flies were selected for wing length over 18 generations, after which their genomic DNA was sequenced.

(B-B') Average wing length for the "big" and "small" flies (B) and the difference between the two population averages (B') over 18 generations of selection. (At generation 10, flies were reared at $19^{\circ} \mathrm{C}$ to slow down their development and span holidays, which leads to increased body size.)

(C) Histogram showing the distribution of wing lengths for the starting fly population (black) and the final big (green) and small (red) populations after 18 generations of selection.

\section{Figure 2: Selection for wing length yields animals of different sizes}

(A) Relative wing area of flies from the 'big' and 'small' populations.

(B) Weight of flies from the 'big' and 'small' populations.

(C-D) Representative pictures of adult males (C) and pupae (D) from the 'small and 'big' populations.

(E) Unlike total body size, embryo size was not significantly different in the two populations selected for wing length. Embryo size, quantified as area occupied on a picture, from flies of the 'big' and 'small' populations.

Error bars: Std. Dev. ${ }^{* * *}$ t-test $<0.001$ 


\section{Figure 3: TOR activity altered in flies selected for size}

(A) Wings of flies selected to be 'small' are composed of cells of smaller size, compared to wings from 'big' flies. Cell size quantified by counting trichomes and calculating area per trichome. $n=10$ for each population.

(B) 'Big' flies have increased total body triglycerides compared to 'small' flies. $n=3 \times 8$ flies for each population.

(C) Western blot analysis reveals that selecting for size causes TOR activity, but not Yorkie activity, to be upregulated during the larval (growth) stages of development, but not in adulthood. TOR activity quantified by phosphorylation of S6 Kinase and 4E-BP. Big "B”, small "s".

Error bars: Std dev. ${ }^{*}$ ttest $<0.05 ;{ }^{* *}$ ttest $<0.01$.

Figure 4: Identification of TOR regulators amongst genes affected by SNPs

(A) Identification of five genes whose knockdown causes reduced TOR-C1 activity in S2 cells, assayed via phosphorylation of S6K on Thr398, a wellestablished TOR-C1 target.

Figure 5: In silico simulation of sorting reveals that the vast majority of differential SNPs between the two populations are 'false positives' or 'passenger mutations'.

(A) Schematic representation of the genetic architecture used for the stochastic modeling. 
(B) In silico simulation of sorting of 300 'big' and 300 'small' flies per generation, assuming 300 SNPs affect size genome-wide, reproduces a gradual divergence in animal size as observed experimentally.

(C-D) In silico simulation reveals that the vast majority of SNPs differentially selected for in the two populations are false positives. The false positive rate (black) and the discovery rate (red) indicated for different statistical threshold cut-offs (C). Even the highest possible Bonferroni-corrected statistical cut-off (that yields a non-zero discovery rate) of $10^{-250}$ leads to an $81 \%$ false positive rate. If instead an enrichment cut-off is used (D), representing how strongly a particular SNP was differentially selected for in the two populations, the most stringent cut-off has a false-positive rate of $65 \%$.

All simulations were run 3 times with identical parameters, and the mean is shown. Error bars: Std Dev.

Figure 6: In silico simulation of sorting identifies parameters required for successfully setting up a "sort-and-sequence" approach.

(A) False-positive and discovery rates resulting from in silico simulations in which two parameters are varied: the number of SNPs genome-wide affecting the trait of interest, as well as the number of flies sorted per generation in each population. As the number of flies sorted increases, the false-positive rate drops due to reduced chances of differentially selecting neutral SNPs by chance.

(B) False-positive rates resulting from in silico simulations with starting populations of differing initial complexity, quantified as the number of floating 
SNPs genome-wide in the starting population. Simulations were run for 200 flies sorted per population per generation, and 100 SNPs affecting size.

(C) Repeating the "sort-and-sequence' experiment in 3 biological replicates can improve the false positive rate, however the number of animals sorted per generation is more important.

All simulations were run 3 times with identical parameters, and the mean is shown. Error bars: Std Dev. 


\section{Strassburger et al. Figure 1}

A

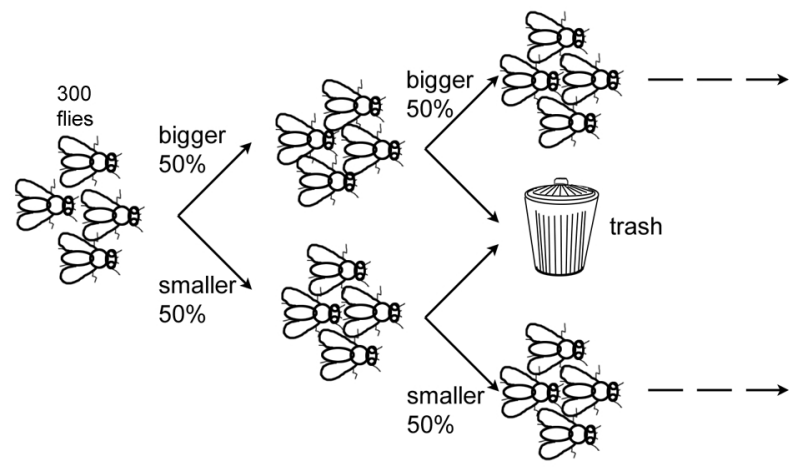

sequence genome

B

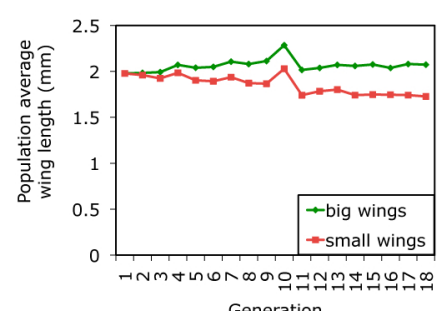

B'

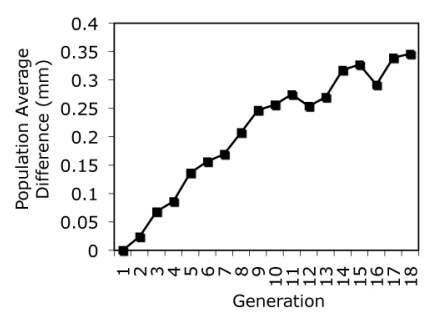

C

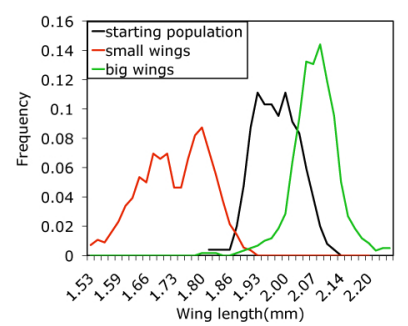




\section{Strassburger et al. Figure 2}

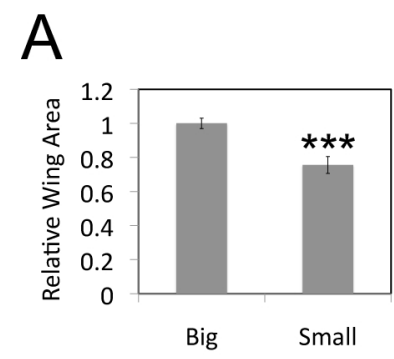

$\mathrm{B}$

C

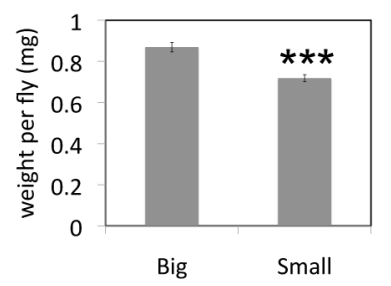

Small

Big

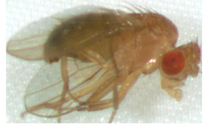

$\mathrm{D}$

\section{$E$}

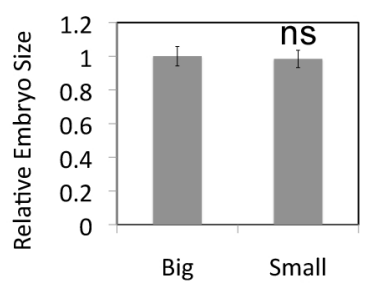




\section{Strassburger et al. Figure 3}

A

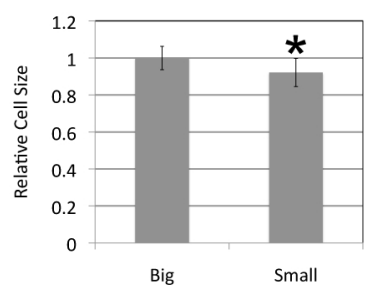

B

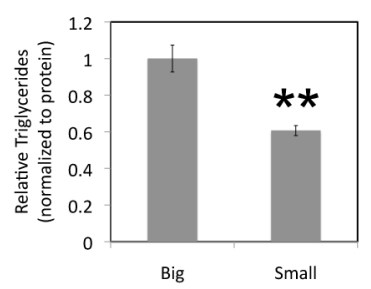

C

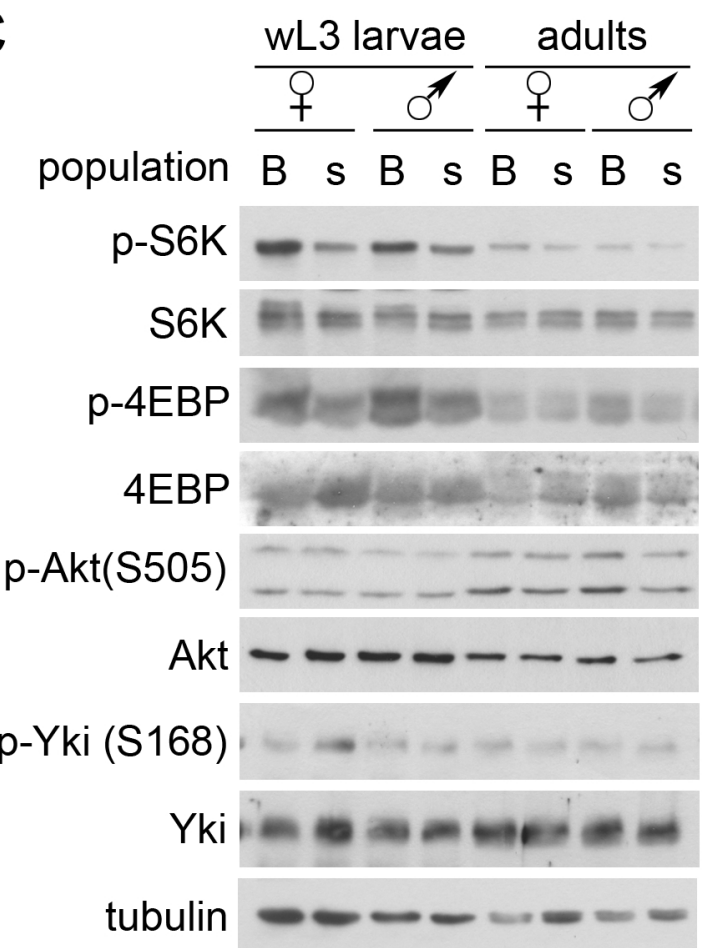




\section{Strassburger et al. Figure 4}

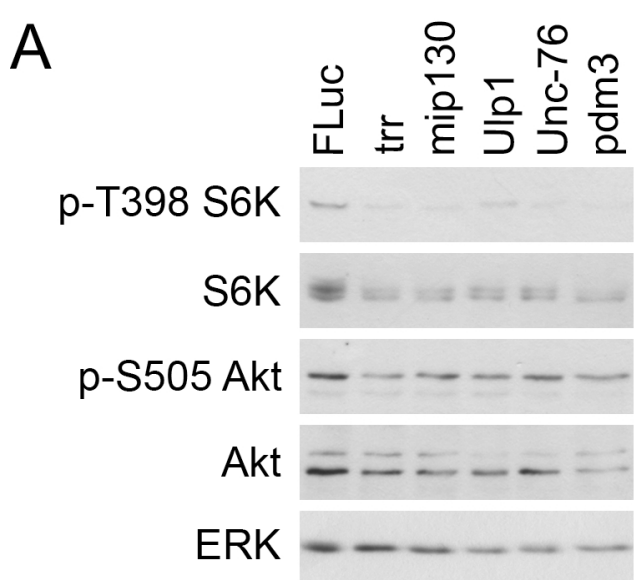




\section{Strassburger et al. Figure 5}

A

size effect

SNPs Chr 1

...Chr 2...Chr 3

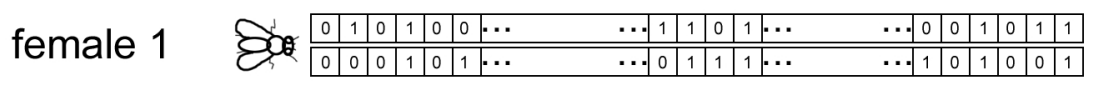

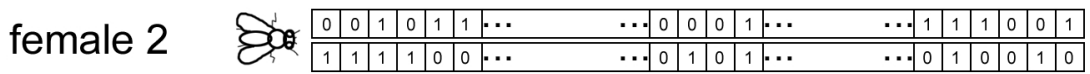

B

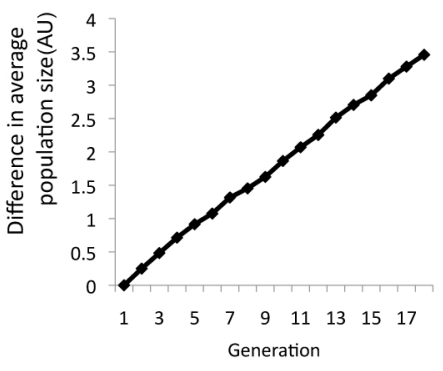

C

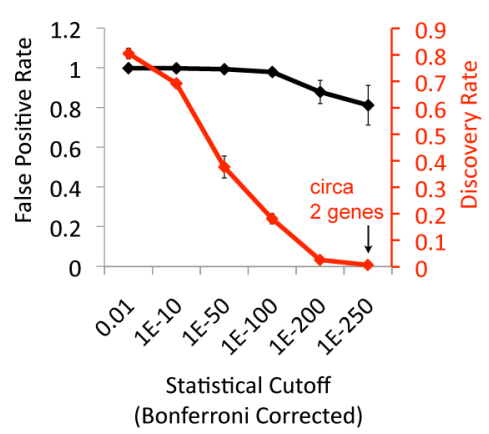

$\mathrm{D}$

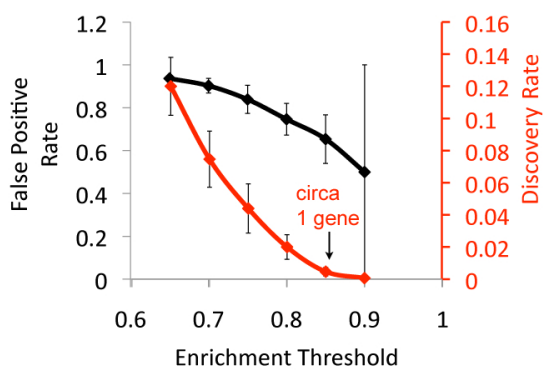


A

\section{Strassburger et al. Figure 6}

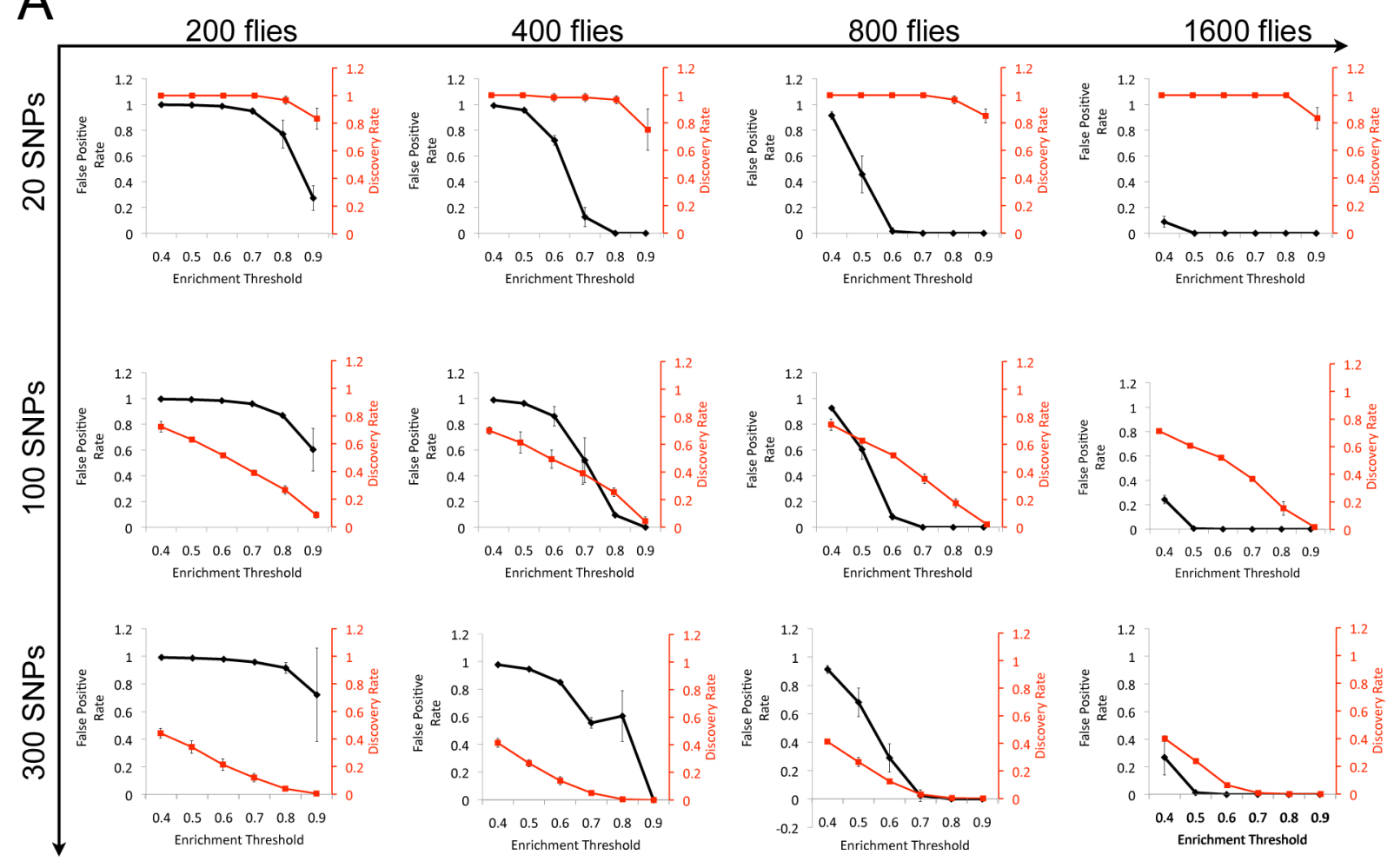

\section{B}

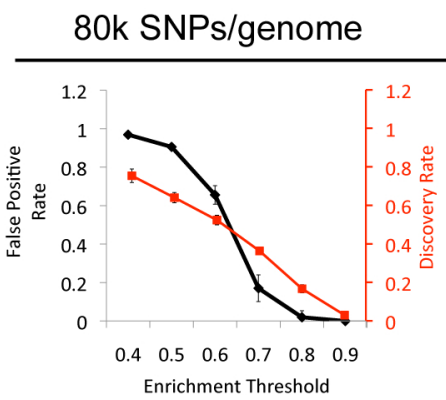

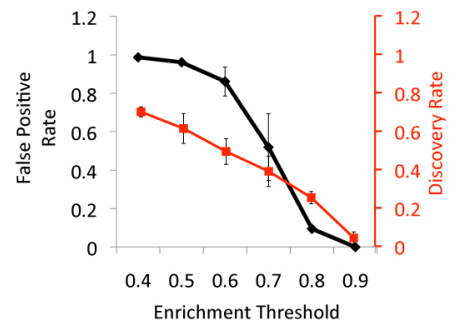

Enrichment Threshold

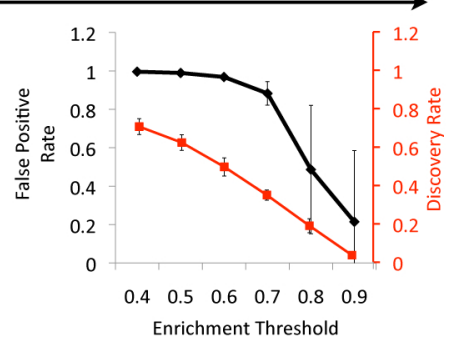

Enrichment Threshold
C

200 flies

per generation per generation

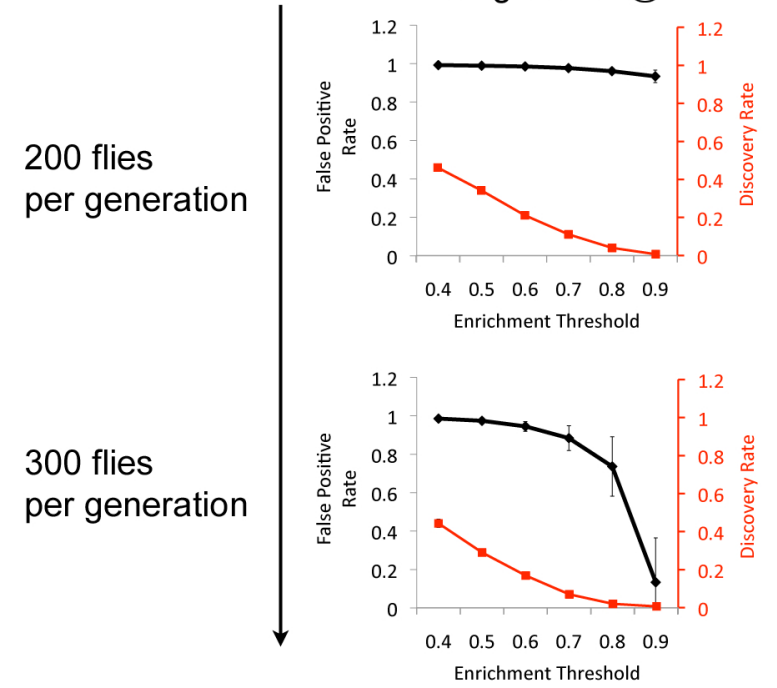

single sort $\bigcirc$

Enrichment Threshold
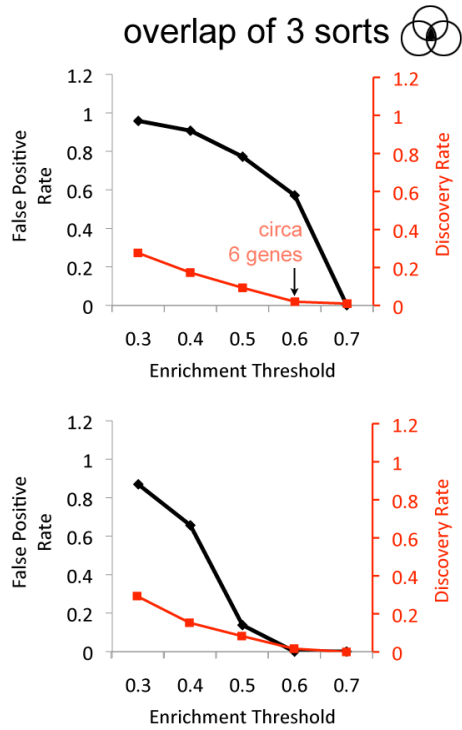
Sorting and Sequencing Flies for Size:

Identification of novel TOR regulators

and Parameters for Successful Sorting

Strassburger et al.

\section{C code of computer simulation}

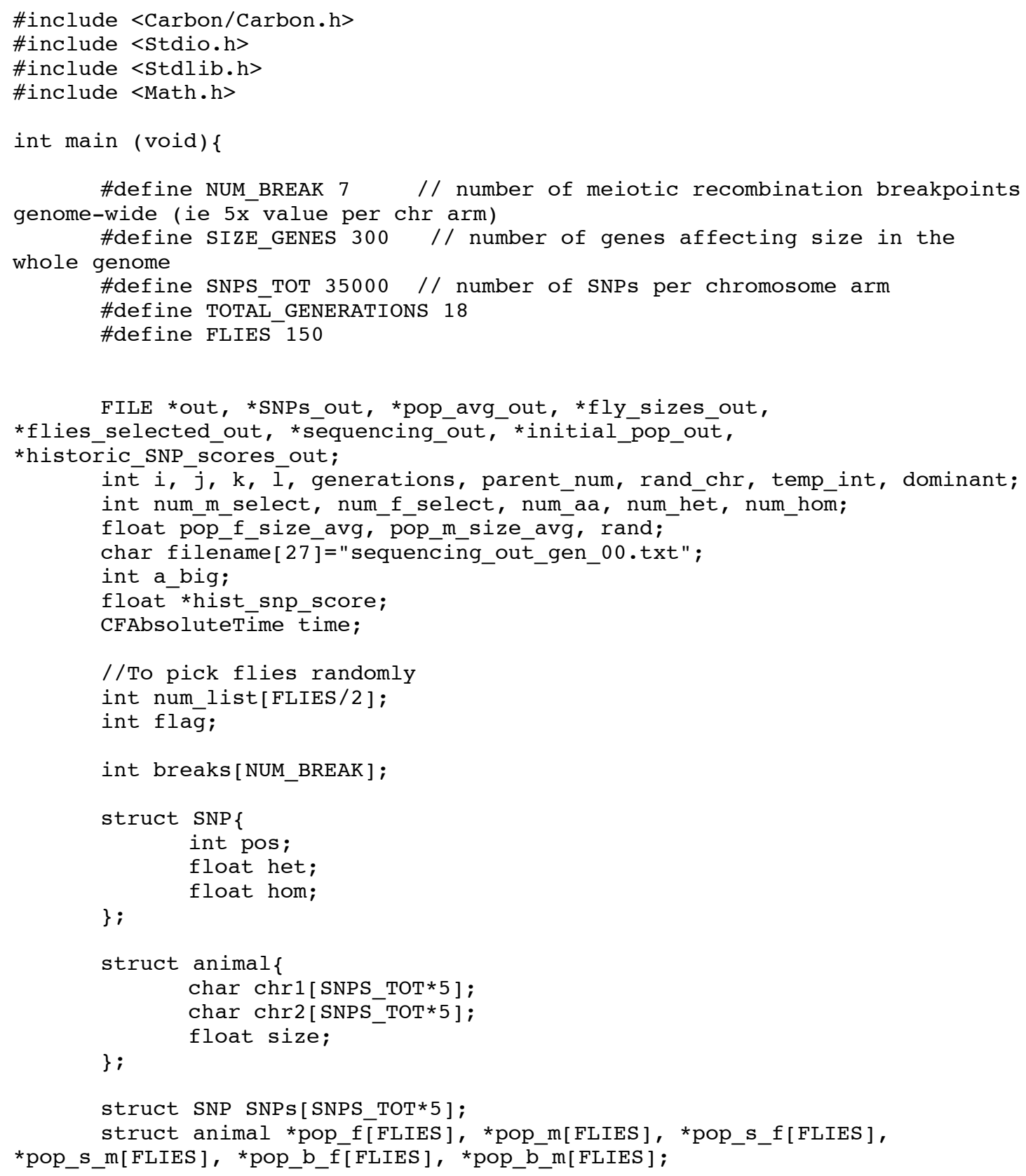


bioRxiv preprint doi: https://doi.org/10.1101/119719; this version posted March 24, 2017. The copyright holder for this preprint (which was not certified by peer review) is the author/funder, who has granted bioRxiv a license to display the preprint in perpetuity. It is made available under aCC-BY-NC-ND 4.0 International license.

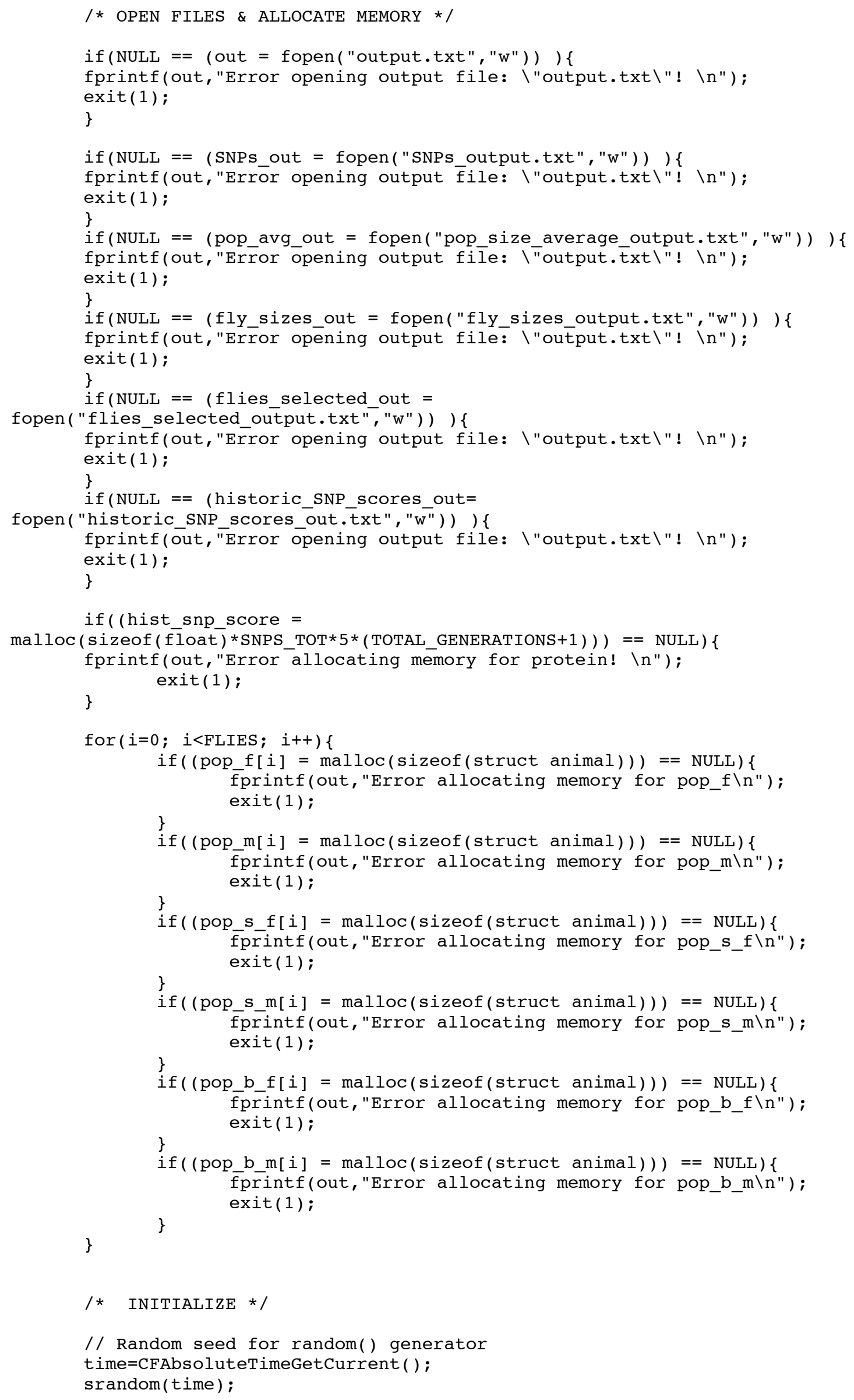


bioRxiv preprint doi: https://doi.org/10.1101/119719; this version posted March 24, 2017. The copyright holder for this preprint (which was

not certified by peer review) is the author/funder, who has granted bioRxiv a license to display the preprint in perpetuity. It is made available under aCC-BY-NC-ND 4.0 International license.

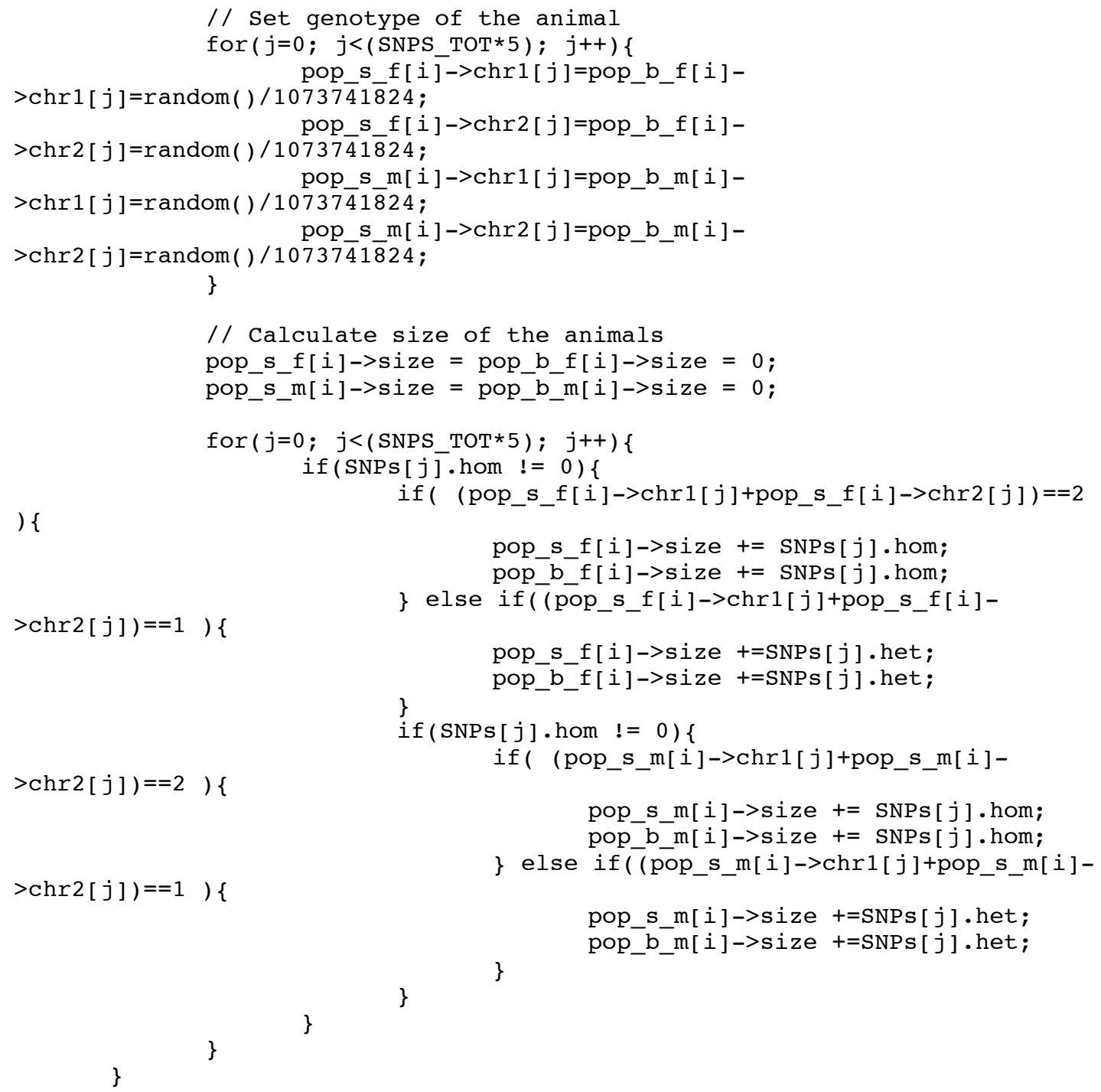

/*****OUTPUT GENOTYPES OF INITIAL POPULATION*****/

if $($ NULL $==$ (initial_pop_out $=$

fopen("initial_population_genotypes_output.txt", "w")) ) \{

fprintf(out,"Error opening output file: \"output.txt\"! \n"); $\operatorname{exit}(1)$;

\}

fprintf(initial_pop_out, "SNP $\backslash t ")$;

for $(i=0 ; i<$ FLIES; $\bar{i} \overline{+}+\{$

fprintf(initial pop out, "Female $\% i$ chr $1 \backslash t$ Female $\% i \operatorname{chr} 2 \backslash t ", i, i)$;

\}

fprintf(initial_pop_out," $\backslash n "$ );

for $(j=0 ; j<$ SNPS TOT; $j++)\{$

if(SNPs $[\bar{j}]$.hom $!=0$ ) fprintf(initial_pop_out, "*");

fprintf(initial pop out, "ChrX-oi $i t ", \bar{j})$;

for $(i=0 ; i<$ FLIES; $i \overline{+}+)\{$ fprintf(initial_pop_out, " $\mathrm{c} \backslash t \% c \backslash t ", 0^{\prime}+$ pop_b_f[i]-

>chr1[j], '0'+pop_b_f[i]->chr2[j]);

\}

\}

fprintf(initial_pop_out," $\backslash \mathrm{n} "$ );

for $(j=$ SNPS_TOT; $j<(3 *$ SNPS_TOT $) ; j++)\{$

if (SNPs[j].hom != $\overline{0}$ ) fprintf(initial_pop_out, "*");

fprintf(initial_pop_out, $" \operatorname{Chr} 2-\% i \backslash t ", \bar{j})$; 
bioRxiv preprint doi: https://doi.org/10.1101/119719; this version posted March 24, 2017. The copyright holder for this preprint (which was not certified by peer review) is the author/funder, who has granted bioRxiv a license to display the preprint in perpetuity. It is made available under aCC-BY-NC-ND 4.0 International license.

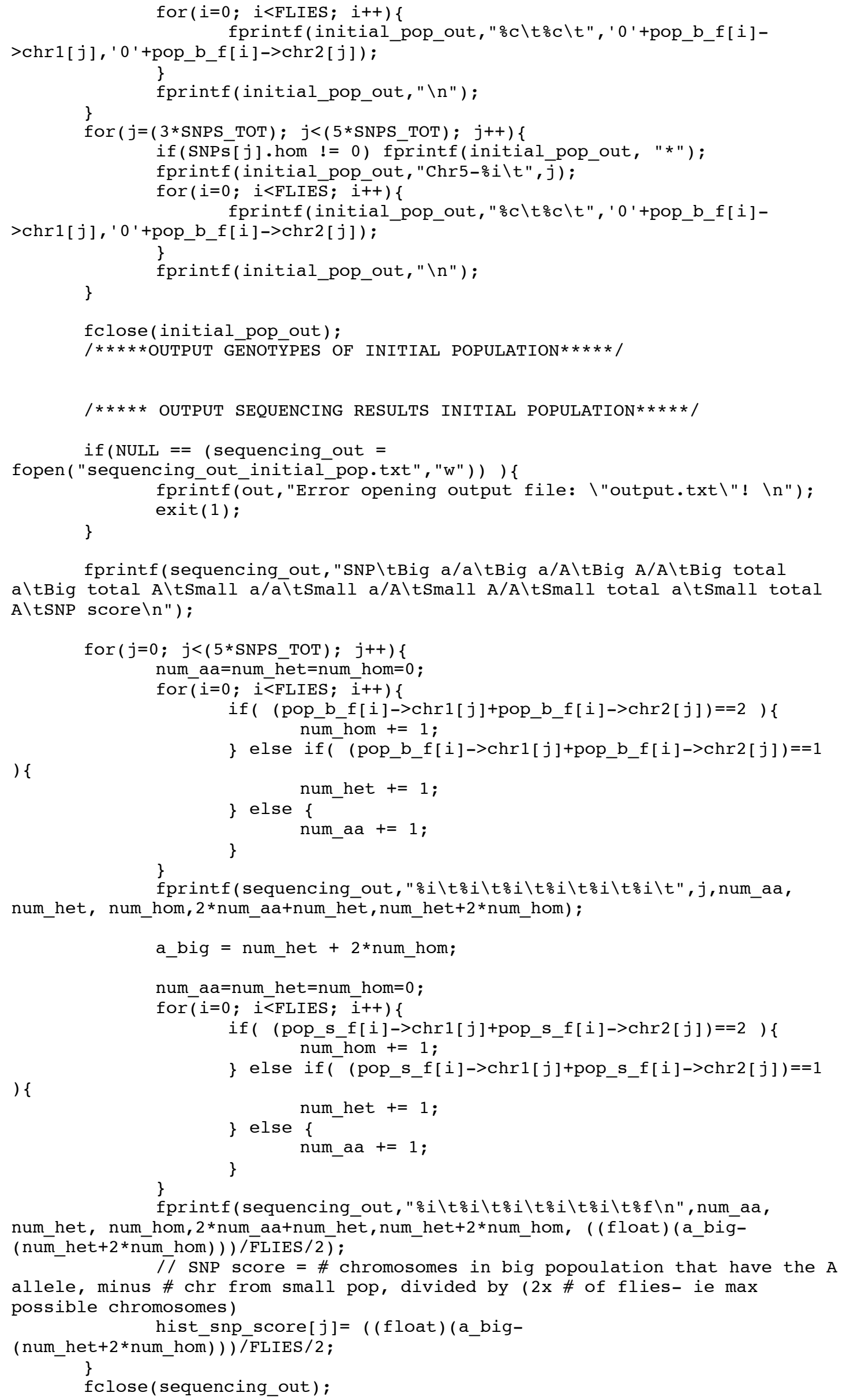


bioRxiv preprint doi: https://doi.org/10.1101/119719; this version posted March 24, 2017. The copyright holder for this preprint (which was

not certified by peer review) is the author/funder, who has granted bioRxiv a license to display the preprint in perpetuity. It is made available under aCC-BY-NC-ND 4.0 International license.

/***** OUTPUT SEQUENCING RESULTS INITIAL POPULATION*****/

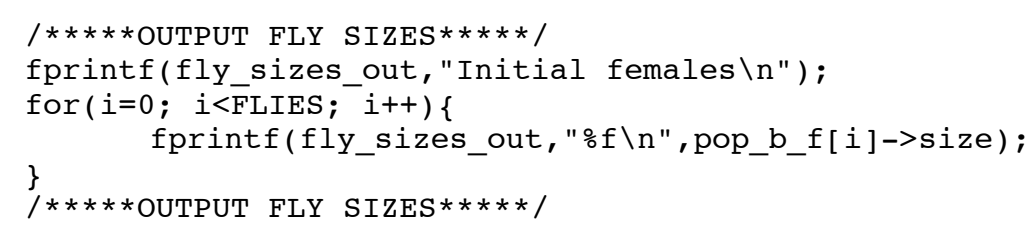

\section{/* EXECUTE SORTing*/}

srandom(time);

fprintf(flies_selected_out,"Generation \tBig females $\backslash$ tBig males $\backslash t$ Small females $\backslash$ tSmall males $\backslash n ")$;

for (generations=0; generations<TOTAL_GENERATIONS ; generations ++ ) \{

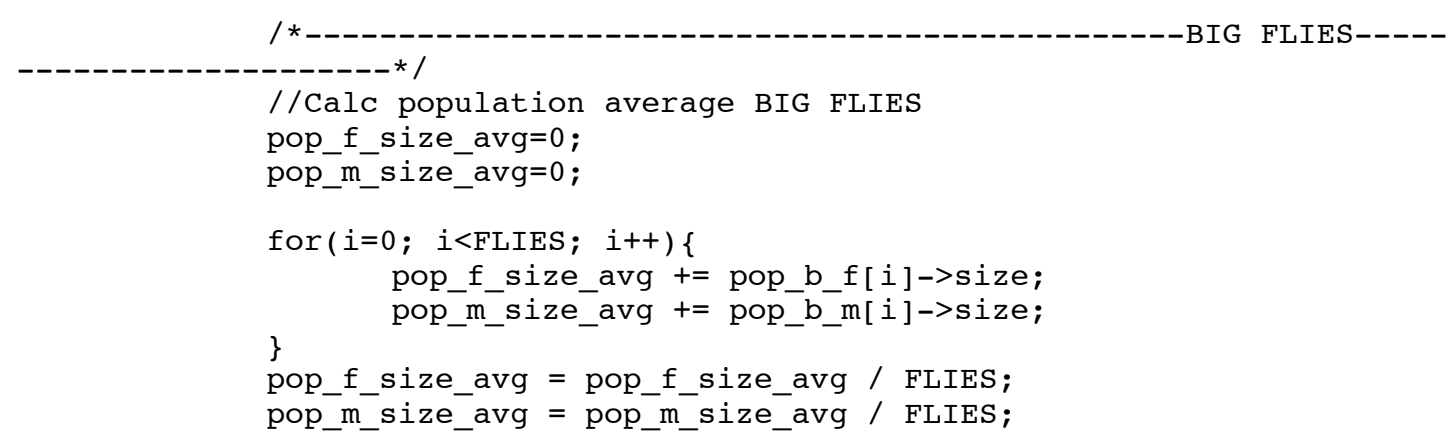

$/ * * * * *$ OUTPUT POPULATION AVERAGE SIZE*****/

fprintf(pop_avg_out," $\% i \backslash t \circ f \backslash t "$,generations,pop_f_size_avg); $/ * * * * *$ OUTPUT POPULATION AVERAGE SIZE $* * * * * /$

\section{//Sort BIG FLIES}

( FLIES) ) \{

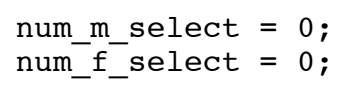


bioRxiv preprint doi: https://doi.org/10.1101/119719; this version posted March 24, 2017. The copyright holder for this preprint (which was

not certified by peer review) is the author/funder, who has granted bioRxiv a license to display the preprint in perpetuity. It is made available under aCC-BY-NC-ND 4.0 International license.

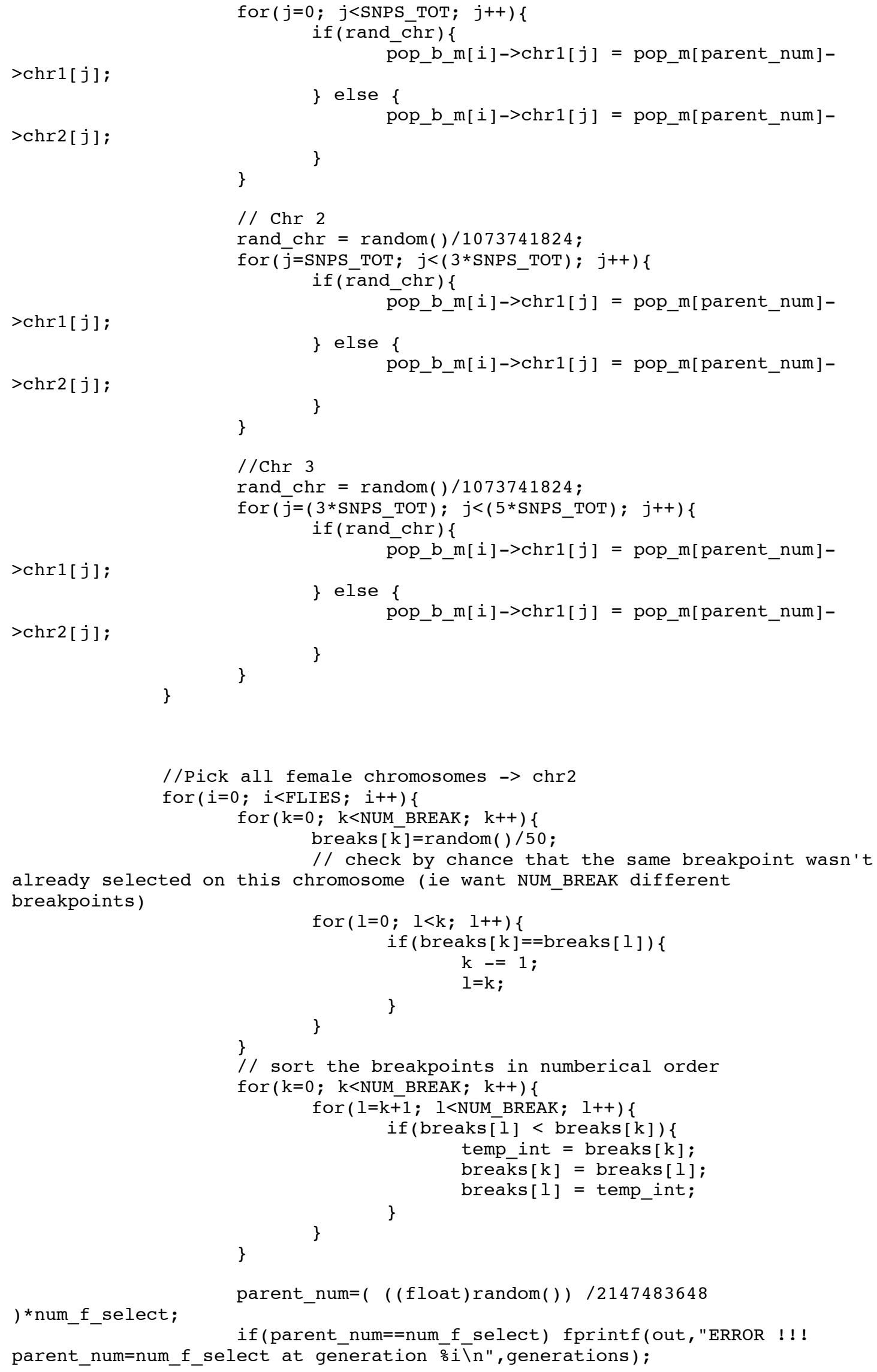


bioRxiv preprint doi: https://doi.org/10.1101/119719; this version posted March 24, 2017. The copyright holder for this preprint (which was

not certified by peer review) is the author/funder, who has granted bioRxiv a license to display the preprint in perpetuity. It is made available under aCC-BY-NC-ND 4.0 International license.

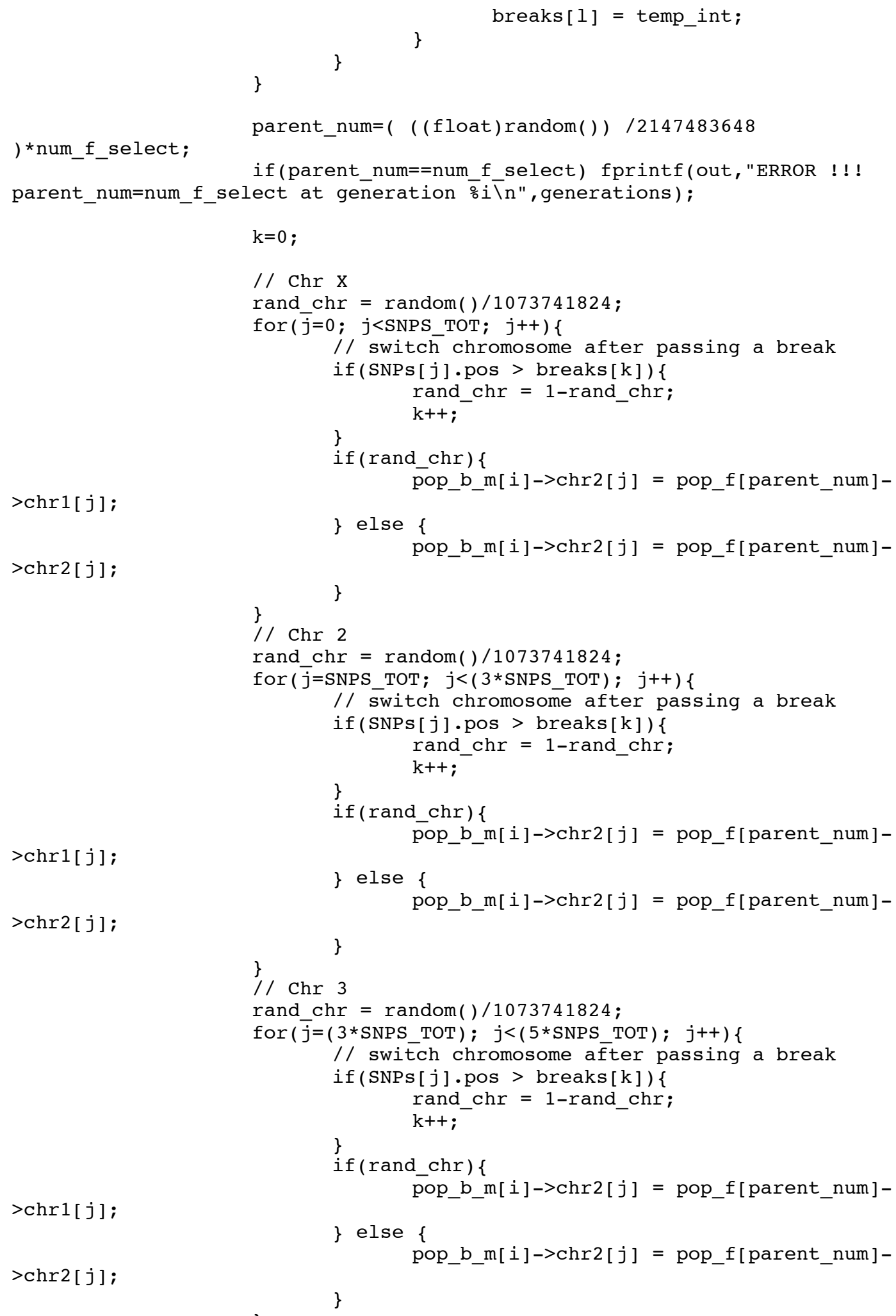


bioRxiv preprint doi: https://doi.org/10.1101/119719; this version posted March 24, 2017. The copyright holder for this preprint (which was

not certified by peer review) is the author/funder, who has granted bioRxiv a license to display the preprint in perpetuity. It is made available under aCC-BY-NC-ND 4.0 International license.

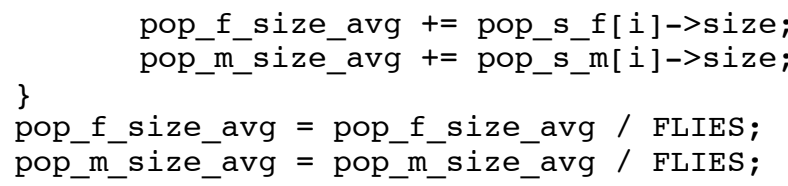

/*****OUTPUT POPULATION AVERAGE SIZE*****/

fprintf(pop_avg_out," $\% \mathrm{f} \backslash \mathrm{n} "$, pop_f_size_avg) ;

/*****OUTPUT POPULATION AVERAGE SIZE*****/

\section{//Sort SMALL FLIES}

num_m_select $=0$;

num_f_select $=0$;

for $(i=0 ; i<$ FLIES; $i++)\{$

< FLIES) ) \{

if((pop_s_f[i]->size <= pop_f_size_avg) \&\& (num_f_select

$>\operatorname{chr} 1[j]=p o p \_s \_f[i]->\operatorname{chr} 1[j]$

for $(j=0 ; j<(5 *$ SNPS_TOT $) ; j++)$ pop_f[num_f_select]-

>chr2[j]=pop_s_f[i]->chr2[j] i for $(j=0 ; j<(5 *$ SNPS_TOT $) ; j++)$ pop_f [num_f_select $]-$ >size=pop_s_f[i]->size;

\} for $(j=0 ; j<(5 *$ SNPS_TOT $) ; j++)$ pop_f[num_f_select]num_f_select $+=1$;

\}

for $(i=0 ; i<$ FLIES; $i++)\{$

(num_m_select $<$ FLIES) $)\{$

if ( pop_s_m[i]->size $<=$ pop_m_size_avg) \&\&

>chr1[j]=pop_s_m[i]->chr1[j] i

for $(j=0 ; j<(5 *$ SNPS_TOT $) ; j++)$ pop_m[num_m_select $]-$

>chr2[j]=pop_s_m[i]->chr2[j] i for $(j=0 ; j<(5 *$ SNPS_TOT $) ; j++)$ pop_m[num_m_select $]-$

>size=pop_s_m[i]->size;

\} for $(j=0 ; j<(5 *$ SNPS_TOT $) ; j++)$ pop_m[num_m_select]num_m_select $+=1$;

\}

\section{$/ * * * *$ OUTPUT FLY SELECTION NUMBERS $* * * * * /$}

fprintf(flies_selected_out," $\% i \backslash t \% i \backslash n "$,num_f_select,num_m_select); $/ * * * *$ OUTPUT FLY SELECTION NUMBERS $\bar{*}$ ***/

)*num_m_select;

//Generate next generation SMALL FLIES

//Pick all male chromosomes (no recombination) -> chr1 for $(i=0 ; i<$ FLIES; $i++)\{$ parent_num $=($ ( (float $)$ random ()$) / 2147483648$

if (parent num==num $m$ select) fprintf (out, "ERROR !!! parent_num=num_m_select at Generation $\bar{\circ} i$ probably due to population reaching homogeneity $\backslash n "$ ", generations );

$>\operatorname{chr} 1[j]$;

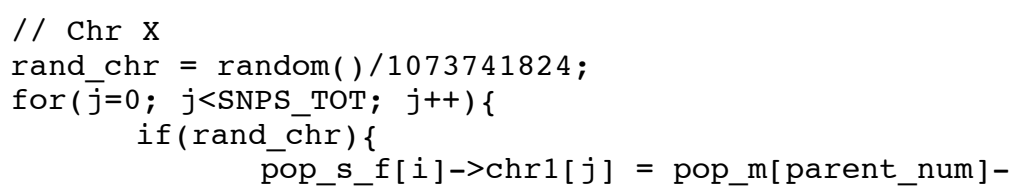


bioRxiv preprint doi: https://doi.org/10.1101/119719; this version posted March 24, 2017. The copyright holder for this preprint (which was

not certified by peer review) is the author/funder, who has granted bioRxiv a license to display the preprint in perpetuity. It is made available under aCC-BY-NC-ND 4.0 International license.

$>\operatorname{chr} 2[j]$;

pop_s_f[i]->chrl[j] = pop_m[parent_num]-

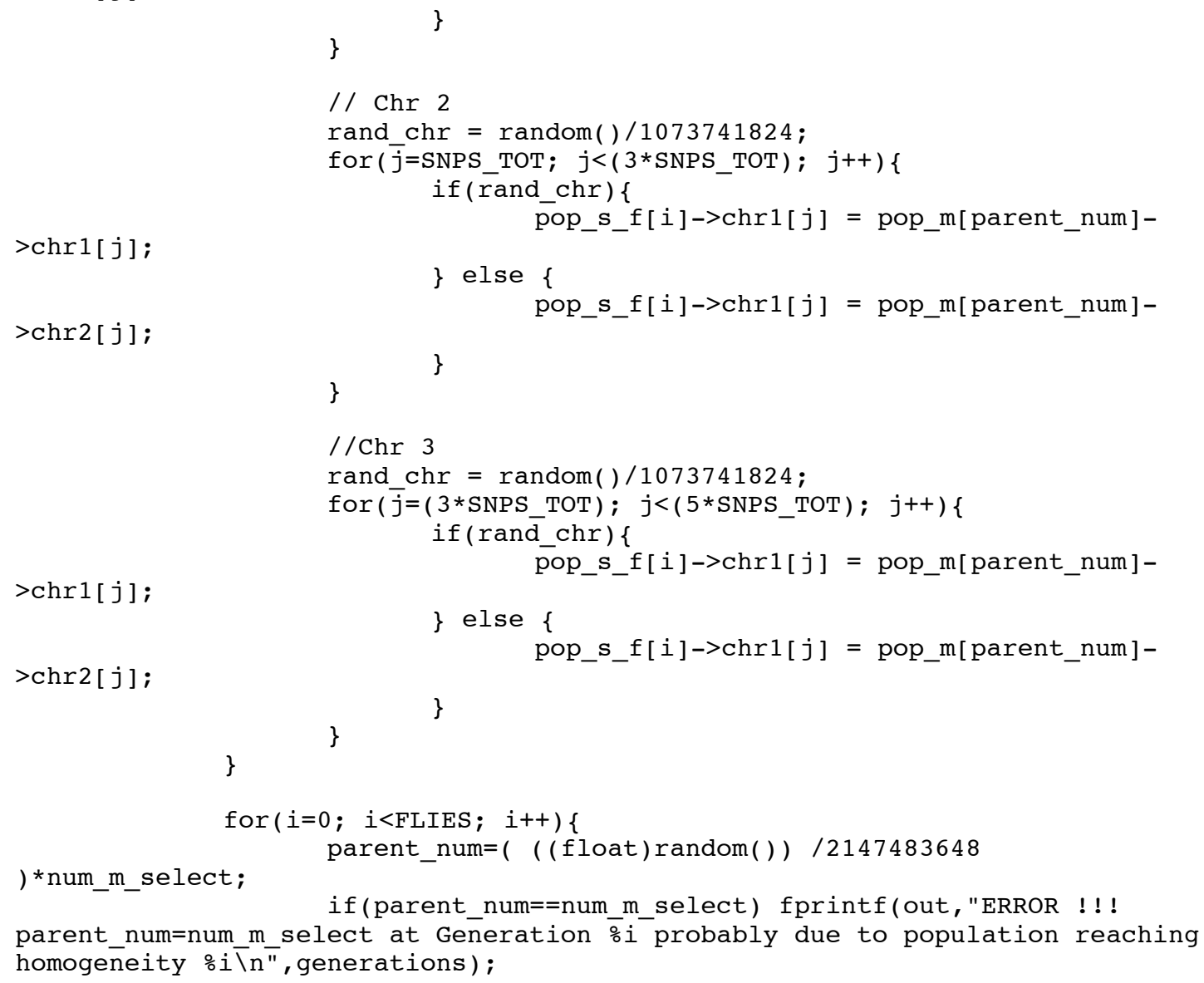


bioRxiv preprint doi: https://doi.org/10.1101/119719; this version posted March 24, 2017. The copyright holder for this preprint (which was

not certified by peer review) is the author/funder, who has granted bioRxiv a license to display the preprint in perpetuity. It is made available under aCC-BY-NC-ND 4.0 International license.

$>\operatorname{chr} 2[j] ;$

pop_s_m[i]->chrl[j] = pop_m[parent_num]-

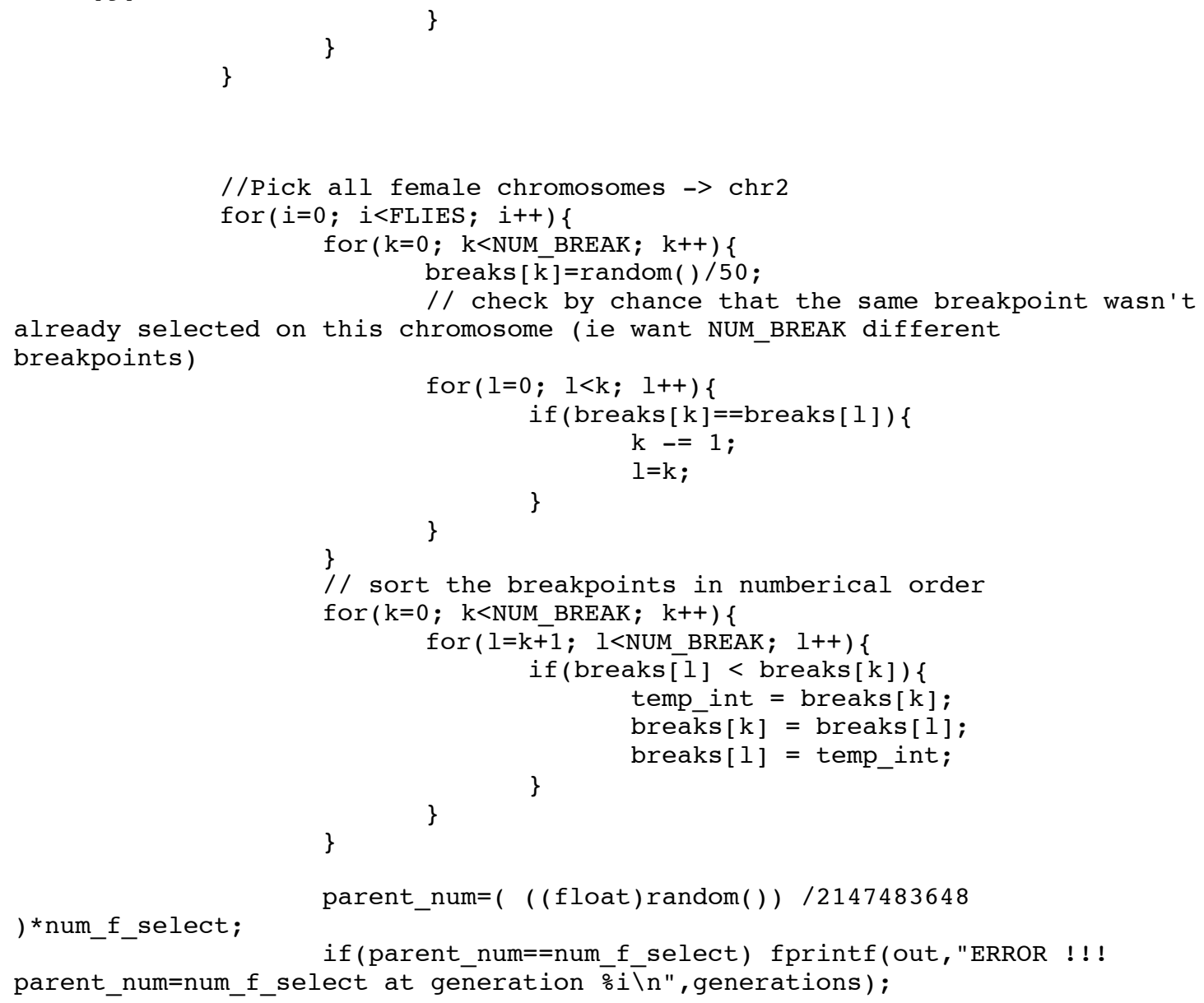


bioRxiv preprint doi: https://doi.org/10.1101/119719; this version posted March 24, 2017. The copyright holder for this preprint (which was

not certified by peer review) is the author/funder, who has granted bioRxiv a license to display the preprint in perpetuity. It is made available under aCC-BY-NC-ND 4.0 International license.

$>\operatorname{chr} 2[j]$;

pop_s_f[i]->chr2[j] = pop_f[parent_num]-

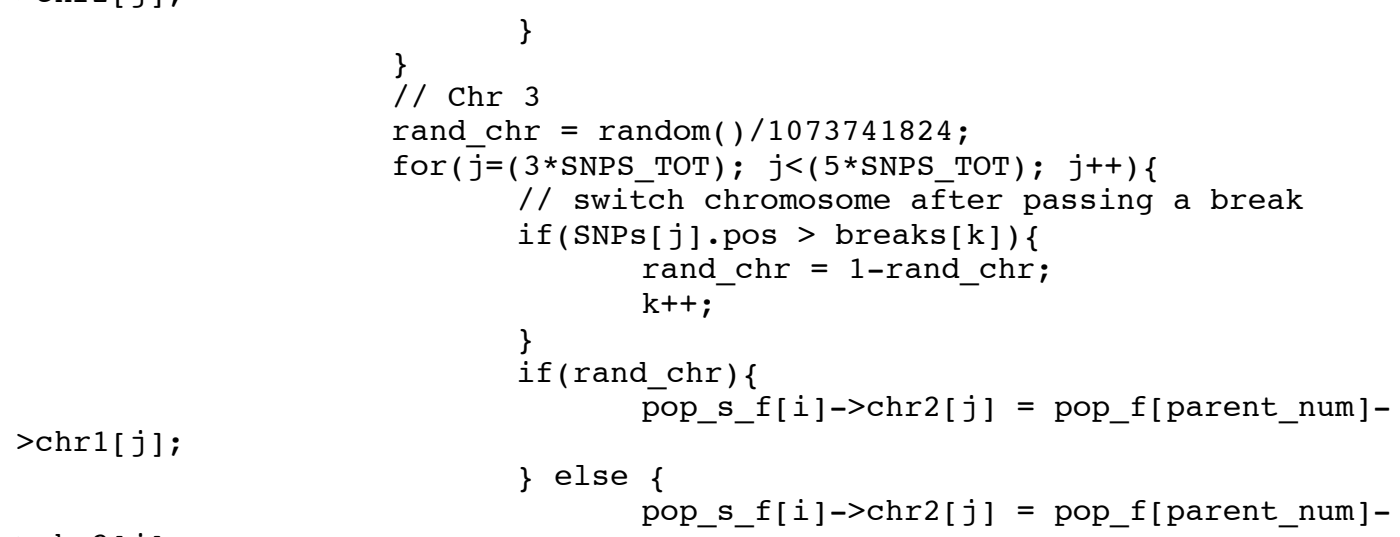


bioRxiv preprint doi: https://doi.org/10.1101/119719; this version posted March 24, 2017. The copyright holder for this preprint (which was

not certified by peer review) is the author/funder, who has granted bioRxiv a license to display the preprint in perpetuity. It is made available under aCC-BY-NC-ND 4.0 International license.

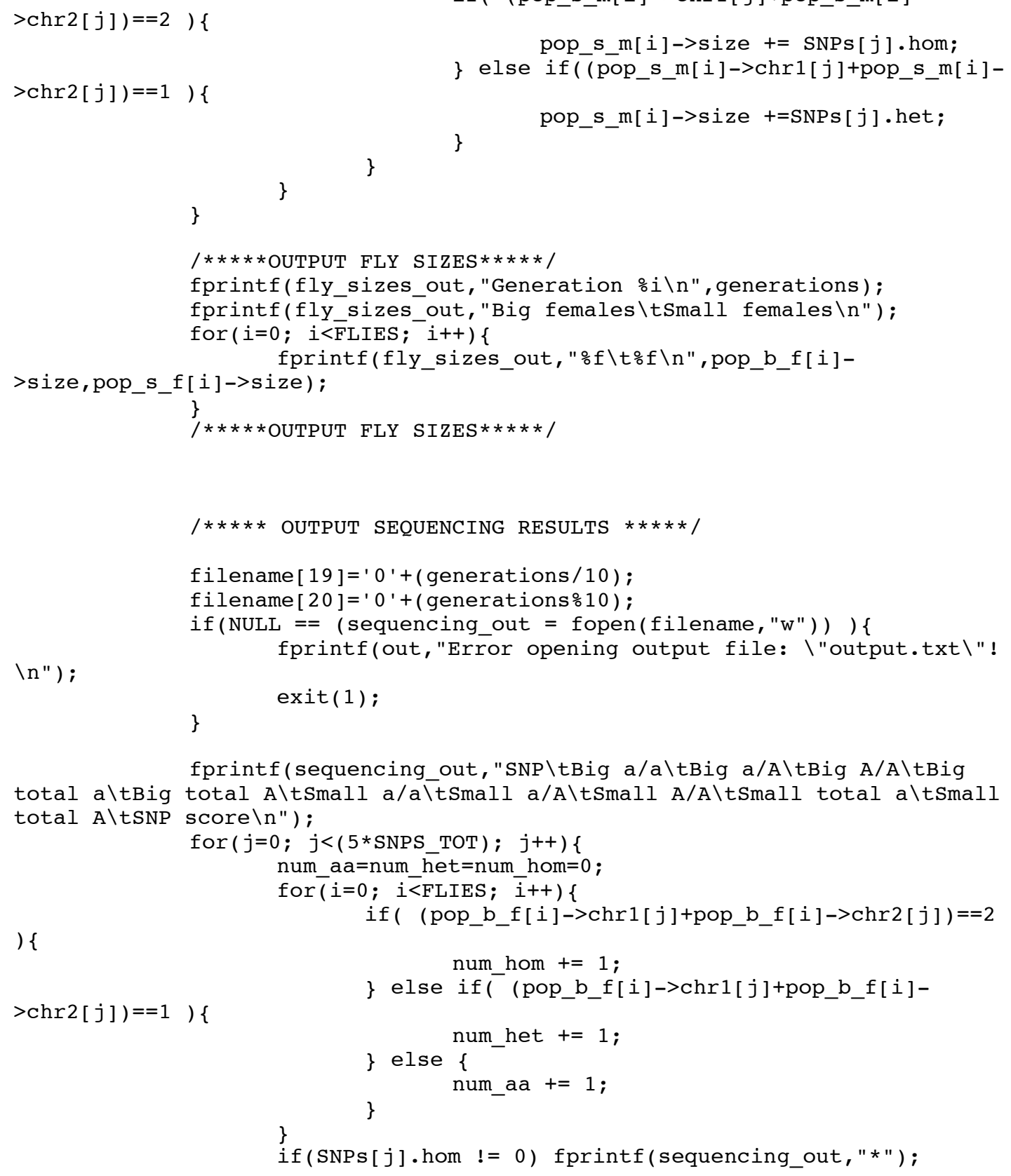

fprintf(sequencing_out," $\% i \backslash t \circ i \backslash t \circ i \backslash t \circ i \backslash t \circ i \backslash t \circ i \backslash t ", j$, num_aa, num_het, num_hom,2*num_aa+num_het,num_het+2*num_hom);

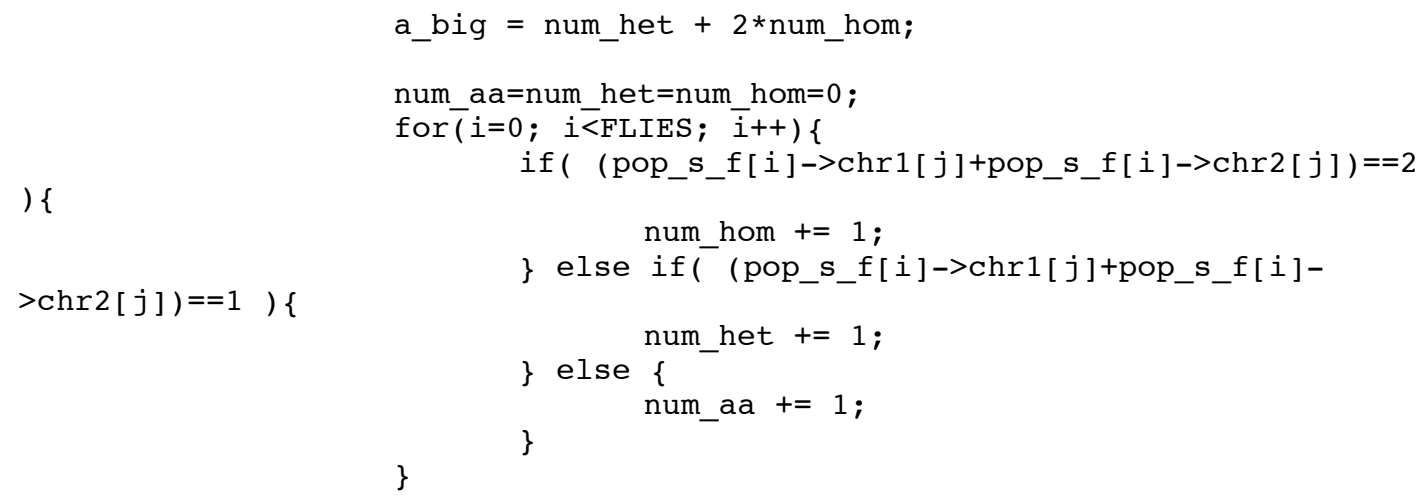




\section{Supplemental Table 1: Positions of Differential SNPs}

Chromosome position

$2 \mathrm{~L}$

$2 \mathrm{~L}$

$2 \mathrm{~L}$

$2 \mathrm{~L}$

$2 \mathrm{~L}$

$2 \mathrm{~L}$

$2 \mathrm{~L}$

$2 \mathrm{~L}$

$2 \mathrm{~L}$

$2 \mathrm{~L}$

$2 \mathrm{~L}$

$2 \mathrm{~L}$

$2 \mathrm{~L}$

2L

$2 \mathrm{~L}$

$2 \mathrm{~L}$

$2 \mathrm{~L}$

$2 \mathrm{~L}$

$2 \mathrm{~L}$

2L

$2 \mathrm{~L}$

$2 \mathrm{~L}$

$2 \mathrm{~L}$

$2 \mathrm{~L}$

$2 \mathrm{~L}$

$2 \mathrm{~L}$

$2 \mathrm{~L}$

$2 \mathrm{~L}$

$2 \mathrm{~L}$

$2 \mathrm{~L}$

2L

$2 \mathrm{~L}$

$2 \mathrm{~L}$

$2 \mathrm{~L}$

$2 \mathrm{~L}$

$2 \mathrm{~L}$

$2 \mathrm{~L}$

$2 \mathrm{~L}$

$2 \mathrm{~L}$

$2 \mathrm{~L}$

$2 \mathrm{~L}$

$2 \mathrm{~L}$

2L

$2 \mathrm{~L}$

$2 \mathrm{~L}$

$2 \mathrm{~L}$

$2 \mathrm{~L}$

$2 \mathrm{~L}$

$2 \mathrm{~L}$

$2 \mathrm{~L}$
372959

529663

555027

674684

747998

760040

761871

907606

1316977

1633751

1852691

1955402

2126661

2204937

2254612

2273379

2379679

2381128

2406752

2420284

2421314

2422426

2537011

2539673

3113130

3121293

3125826

3125841

3126036

3141089

3145302

3184743

3240836

3276018

3407409

3472150

3585490

3611433

3724848

3729928

3777454

3820880

3873893

4209604

4231126

4292861

4301661

4320671

4339602

4403288 difference

BIG coverage small coverag $€ \mathrm{p}$-score

$\begin{array}{lr}2 & 5 \\ 2 & 12 \\ 2 & 5 \\ 2 & 4 \\ 2 & 5\end{array}$

5
12
5
4
5

13

10

11

5

5

6

7

10

12

11

6

8

7

7

4

4

7

5

6

6

5

6

5

4

5

16

5

6

6

5

8

5

5

5

5

4

11

6

9

6

6

5

5
19

4

12

9

15

15

16

0

0

0

0

0

0

0

0

0

0

0

0

0

0

0

0

0

0

0

0

0

0

0

0

110

$5 \quad 0$

$6 \quad 0$

$8 \quad 0$

110

$5 \quad 0$

110

50

$5 \quad 0$

$5 \quad 0$

$6 \quad 0$

$5 \quad 0$

$7 \quad 0$

$6 \quad 0$

$8 \quad 0$

120

110

50

90

50

$6 \quad 0$

$9 \quad 0$

12

5

6 
$2 \mathrm{~L}$

$2 \mathrm{~L}$

$2 \mathrm{~L}$

$2 \mathrm{~L}$

$2 \mathrm{~L}$

$2 \mathrm{~L}$

$2 \mathrm{~L}$

$2 \mathrm{~L}$

2L

$2 \mathrm{~L}$

2L

$2 \mathrm{~L}$

$2 \mathrm{~L}$

2L

$2 \mathrm{~L}$

$2 \mathrm{~L}$

$2 \mathrm{~L}$

$2 \mathrm{~L}$

$2 \mathrm{~L}$

$2 \mathrm{~L}$

$2 \mathrm{~L}$

$2 \mathrm{~L}$

$2 \mathrm{~L}$

$2 \mathrm{~L}$

$2 \mathrm{~L}$

$2 \mathrm{~L}$

$2 \mathrm{~L}$

$2 \mathrm{~L}$

$2 \mathrm{~L}$

$2 \mathrm{~L}$

$2 \mathrm{~L}$

$2 \mathrm{~L}$

2L

2L

$2 \mathrm{~L}$

$2 \mathrm{~L}$

$2 \mathrm{~L}$

$2 \mathrm{~L}$

$2 \mathrm{~L}$

$2 \mathrm{~L}$

$2 \mathrm{~L}$

$2 \mathrm{~L}$

$2 \mathrm{~L}$

$2 \mathrm{~L}$

$2 \mathrm{~L}$

2L

$2 \mathrm{~L}$

$2 \mathrm{~L}$

$2 \mathrm{~L}$

$2 \mathrm{~L}$

$2 \mathrm{~L}$

$2 \mathrm{~L}$

$2 \mathrm{~L}$
4417855

4486067

4706540

4730160

7194640

7288005

7443361

7922249

7974791

8467435

8508568

8580810

8587578

8614811

8624367

8634207

8762613

8765945

8779463

8780836

8810549

8810575

8824047

8871626

8927320

8967452

8981467

8989508

8994799

8999857

9048671

9064559

9098696

9101135

9114595

9150191

9209937

9214460

9235267

9261590

9302319

9321925

9329616

9333483

9344218

9364788

9387371

9457474

9504991

9527431

9600530

9608716

9611121
2

2

2

2

2

2

2

2

2

2

2

2

2

2

2

2

2

2

2

2

2

2

2

2

2

2

2

2

2

2

2

2

2

2

2

2

2

2

2

2

2

2

2

2

2

2

2

2

2

2

2

2

2
4
7
5
4
5
9
8
6

10

5

7

5

7

5

4

4

5

5

17

6

5

14

6

4

7

5

4

7

4

5

5

7

8

11

6

8

8

6

7

9

5

5

7

5

9

8

15

6

5

4

10

4

8
9

10

5

9

5

8

11

5

8

7

6

5

7
11

9

9

7

12

7

5

8

4

7

9

13

11

10

5

9

24

13

5
7

10

9

6

11

8

7

17

15

12

24

6

5

9

4

8

5

9

16

11

18

0

0

0

0

0

0

0

0

0

0

0

0

0

0

0

0

0

0

0

0

0

0

0

0

0

0

0

0

0 


\begin{tabular}{|c|c|}
\hline $2 \mathrm{~L}$ & 9633396 \\
\hline $2 \mathrm{~L}$ & 9779551 \\
\hline $2 \mathrm{~L}$ & 9784086 \\
\hline $2 \mathrm{~L}$ & 9882920 \\
\hline $2 \mathrm{~L}$ & 9911954 \\
\hline $2 \mathrm{~L}$ & 9957014 \\
\hline $2 \mathrm{~L}$ & 10011711 \\
\hline $2 \mathrm{~L}$ & 10015466 \\
\hline $2 \mathrm{~L}$ & 10017656 \\
\hline $2 \mathrm{~L}$ & 10020574 \\
\hline $2 \mathrm{~L}$ & 10020602 \\
\hline $2 \mathrm{~L}$ & 10049066 \\
\hline $2 \mathrm{~L}$ & 10136914 \\
\hline $2 \mathrm{~L}$ & 10151359 \\
\hline $2 \mathrm{~L}$ & 10152808 \\
\hline $2 \mathrm{~L}$ & 10165520 \\
\hline $2 \mathrm{~L}$ & 10178968 \\
\hline $2 \mathrm{~L}$ & 10199877 \\
\hline $2 \mathrm{~L}$ & 10212301 \\
\hline $2 \mathrm{~L}$ & 10212872 \\
\hline $2 \mathrm{~L}$ & 10250259 \\
\hline $2 \mathrm{~L}$ & 10252260 \\
\hline $2 \mathrm{~L}$ & 10307866 \\
\hline $2 \mathrm{~L}$ & 10838949 \\
\hline $2 \mathrm{~L}$ & 13695737 \\
\hline $2 \mathrm{~L}$ & 18748989 \\
\hline $2 R$ & 883056 \\
\hline $2 \mathrm{R}$ & 3915796 \\
\hline $2 \mathrm{R}$ & 3921584 \\
\hline $2 R$ & 3926376 \\
\hline $2 \mathrm{R}$ & 3927715 \\
\hline $2 \mathrm{R}$ & 3928319 \\
\hline $2 R$ & 3928457 \\
\hline $2 R$ & 3931643 \\
\hline $2 \mathrm{R}$ & 3932881 \\
\hline $2 R$ & 3935024 \\
\hline $2 \mathrm{R}$ & 3935141 \\
\hline $2 \mathrm{R}$ & 3935573 \\
\hline $2 R$ & 3936788 \\
\hline $2 \mathrm{R}$ & 3944833 \\
\hline $2 R$ & 3948365 \\
\hline $2 R$ & 3948894 \\
\hline $2 \mathrm{R}$ & 3949215 \\
\hline $2 R$ & 3949671 \\
\hline $2 R$ & 3950717 \\
\hline $2 \mathrm{R}$ & 3954596 \\
\hline $2 R$ & 3956824 \\
\hline $2 R$ & 3959542 \\
\hline $2 \mathrm{R}$ & 3968849 \\
\hline $2 \mathrm{R}$ & 3969167 \\
\hline $2 R$ & 3969297 \\
\hline $2 R$ & 3969573 \\
\hline . & 3971783 \\
\hline
\end{tabular}

\begin{tabular}{|c|c|}
\hline \multirow{2}{*}{$\begin{array}{r}10 \\
4\end{array}$} & 4 \\
\hline & 10 \\
\hline 11 & 10 \\
\hline 5 & 5 \\
\hline 6 & 21 \\
\hline 5 & 6 \\
\hline 10 & 13 \\
\hline 4 & 19 \\
\hline 4 & 14 \\
\hline 7 & 6 \\
\hline 6 & 10 \\
\hline 8 & 12 \\
\hline 9 & 5 \\
\hline 4 & 9 \\
\hline 6 & 13 \\
\hline 5 & 7 \\
\hline 4 & 11 \\
\hline 4 & 9 \\
\hline 4 & 11 \\
\hline 5 & 5 \\
\hline 6 & 10 \\
\hline 5 & 5 \\
\hline 8 & 7 \\
\hline 5 & 6 \\
\hline 5 & 11 \\
\hline 6 & 11 \\
\hline 6 & 5 \\
\hline 8 & 8 \\
\hline 7 & 5 \\
\hline 5 & 28 \\
\hline 4 & 13 \\
\hline 32 & 13 \\
\hline 5 & 7 \\
\hline 5 & 18 \\
\hline 23 & 19 \\
\hline 8 & 32 \\
\hline 4 & 22 \\
\hline 21 & 15 \\
\hline 9 & 5 \\
\hline 13 & 14 \\
\hline 8 & 8 \\
\hline 9 & 8 \\
\hline 17 & 5 \\
\hline 11 & 11 \\
\hline 6 & 8 \\
\hline 10 & 5 \\
\hline 12 & 5 \\
\hline 9 & 9 \\
\hline 18 & 6 \\
\hline 11 & 4 \\
\hline 9 & 4 \\
\hline 6 & 10 \\
\hline 7 & 5 \\
\hline
\end{tabular}




$\begin{array}{ll}2 R & 3972864 \\ 2 R & 3974155 \\ 2 R & 3975602 \\ 2 R & 4001944 \\ 2 R & 4005880 \\ 2 R & 4010646 \\ 2 R & 4016702 \\ 2 R & 4027965 \\ 2 R & 4030731 \\ 2 R & 4038931 \\ 2 R & 4039111 \\ 2 R & 4039991 \\ 2 R & 4040628 \\ 2 R & 4041476 \\ 2 R & 4041948 \\ 2 R & 4042093 \\ 2 R & 4043058 \\ 2 R & 4045370 \\ 2 R & 4051169 \\ 2 R & 4053115 \\ 2 R & 4058718 \\ 2 R & 4063232 \\ 2 R & 4063447 \\ 2 R & 4063546 \\ 2 R & 4066186 \\ 2 R & 4069126 \\ 2 R & 4069389 \\ 2 R & 4071886 \\ 2 R & 4072483 \\ 2 R & 4072681 \\ 2 R & 4074894 \\ 2 R & 4075154 \\ 2 R & 4075479 \\ 2 R & 4076676 \\ 2 R & 4080366 \\ 2 R & 4080711 \\ 2 R & 4082191 \\ 2 R & 4083436 \\ 2 R & 4086408 \\ 2 R & 4087268 \\ 2 R & 4087390 \\ 2 R & 4089530 \\ 2 R & 4089915 \\ 2 R & 4092182 \\ 2 R & 4092387 \\ 2 R & 4095130 \\ 2 R & 4099635 \\ 2 R & 4099688 \\ 2 R & 4099819 \\ 2 R & \\ 2 R & 4103337 \\ 2 R & \end{array}$

\section{2}

2

2

2

2

2

2

2

2

2

2

2

2

2

2

2

2

2

2

2

2

2

2

2

2

2

2

2

2

2

2

2

2

2

2

2

2

2

2

2

2

2

2

2

2

2

2

2

2

2

2

2

2
11

20

16

7

10

29

15

19

27

8

9

22

17

17

17

14

26

16

9

16

26

4

17

4

9

12

19

5

9

16

6

5

21

12

14

10

8

14

4

9

8

11

15

11

20

5

26

31

11

24

29

7

10
6

8

11

5

12

22

4

18

9

5

8

15

14

4

11

4

16

31

4

4

9

15

25

12

14

5

12

6

16

9

6

16

26

5

6

10

6

6

23

4

12

7

5

17

7

8

9

8

8

10

25

13

24
0

0

0

0

0

0

0

0

0

0

0

0

0

0

0

0

0

0

0

0

0

0

0

0

0

0

0

0

0

0

0

0

0

0

0

0

0

0

0

0

0

0

0

0

0
0

0

0

0 


\begin{tabular}{|c|c|c|c|c|c|}
\hline $2 \mathrm{R}$ & 4112833 & 2 & 10 & 6 & 0 \\
\hline $2 \mathrm{R}$ & 4112996 & 2 & 37 & 24 & 0 \\
\hline $2 \mathrm{R}$ & 4114645 & 2 & 9 & 34 & 0 \\
\hline $2 R$ & 4115014 & 2 & 9 & 17 & 0 \\
\hline $2 \mathrm{R}$ & 4116407 & 2 & 25 & 16 & 0 \\
\hline $2 \mathrm{R}$ & 4116851 & 2 & 9 & 10 & 0 \\
\hline $2 \mathrm{R}$ & 4119375 & 2 & 13 & 6 & 0 \\
\hline $2 \mathrm{R}$ & 4121667 & 2 & 24 & 4 & 0 \\
\hline $2 \mathrm{R}$ & 4122424 & 2 & 13 & 5 & 0 \\
\hline $2 \mathrm{R}$ & 4123001 & 2 & 10 & 4 & 0 \\
\hline $2 R$ & 4123485 & 2 & 15 & 7 & 0 \\
\hline $2 R$ & 4125976 & 2 & 19 & 9 & 0 \\
\hline $2 R$ & 4128568 & 2 & 16 & 13 & 0 \\
\hline $2 \mathrm{R}$ & 4129353 & 2 & 22 & 13 & 0 \\
\hline $2 R$ & 4131123 & 2 & 4 & 16 & 0 \\
\hline $2 \mathrm{R}$ & 4134149 & 2 & 27 & 8 & 0 \\
\hline $2 \mathrm{R}$ & 4134924 & 2 & 17 & 8 & 0 \\
\hline $2 \mathrm{R}$ & 4135038 & 2 & 20 & 5 & 0 \\
\hline $2 R$ & 4135687 & 2 & 26 & 8 & 0 \\
\hline $2 \mathrm{R}$ & 4135787 & 2 & 6 & 19 & 0 \\
\hline $2 \mathrm{R}$ & 4136152 & 2 & 23 & 4 & 0 \\
\hline $2 R$ & 4137681 & 2 & 11 & 5 & 0 \\
\hline $2 \mathrm{R}$ & 4139607 & 2 & 9 & 4 & 0 \\
\hline $2 R$ & 4140177 & 2 & 14 & 9 & 0 \\
\hline $2 \mathrm{R}$ & 4141187 & 2 & 14 & 7 & 0 \\
\hline $2 \mathrm{R}$ & 4142597 & 2 & 19 & 4 & 0 \\
\hline $2 \mathrm{R}$ & 4143233 & 2 & 26 & 6 & 0 \\
\hline $2 R$ & 4143388 & 2 & 11 & 12 & 0 \\
\hline $2 \mathrm{R}$ & 4145741 & 2 & 4 & 21 & 0 \\
\hline $2 \mathrm{R}$ & 4146304 & 2 & 21 & 9 & 0 \\
\hline $2 R$ & 4146345 & 2 & 16 & 8 & 0 \\
\hline $2 \mathrm{R}$ & 4146450 & 2 & 10 & 7 & 0 \\
\hline $2 \mathrm{R}$ & 4146906 & 2 & 14 & 5 & 0 \\
\hline $2 \mathrm{R}$ & 4148155 & 2 & 14 & 7 & 0 \\
\hline $2 \mathrm{R}$ & 4148268 & 2 & 9 & 18 & 0 \\
\hline $2 R$ & 4148737 & 2 & 5 & 31 & 0 \\
\hline $2 R$ & 4148763 & 2 & 9 & 28 & 0 \\
\hline $2 \mathrm{R}$ & 4155357 & 2 & 6 & 7 & 0 \\
\hline $2 \mathrm{R}$ & 4156587 & 2 & 8 & 35 & 0 \\
\hline $2 \mathrm{R}$ & 4157198 & 2 & 15 & 5 & 0 \\
\hline $2 \mathrm{R}$ & 4157261 & 2 & 7 & 6 & 0 \\
\hline $2 \mathrm{R}$ & 4158233 & 2 & 13 & 5 & 0 \\
\hline $2 R$ & 4158282 & 2 & 14 & 8 & 0 \\
\hline $2 \mathrm{R}$ & 4158298 & 2 & 13 & 6 & 0 \\
\hline $2 \mathrm{R}$ & 4158391 & 2 & 5 & 7 & 0 \\
\hline $2 \mathrm{R}$ & 4158540 & 2 & 9 & 4 & 0 \\
\hline $2 R$ & 4159196 & 2 & 20 & 7 & 0 \\
\hline $2 R$ & 4162197 & 2 & 16 & 9 & 0 \\
\hline $2 R$ & 4162785 & 2 & 8 & 20 & 0 \\
\hline $2 \mathrm{R}$ & 4163113 & 2 & 7 & 12 & 0 \\
\hline $2 \mathrm{R}$ & 4163585 & 2 & 9 & 20 & 0 \\
\hline $2 \mathrm{R}$ & 4163760 & 2 & 5 & 15 & 0 \\
\hline $2 R$ & 4164645 & 2 & 4 & 13 & 0 \\
\hline
\end{tabular}




\begin{tabular}{|c|c|}
\hline $2 \mathrm{R}$ & 4164790 \\
\hline $2 \mathrm{R}$ & 4165617 \\
\hline $2 \mathrm{R}$ & 4166846 \\
\hline $2 \mathrm{R}$ & 4168365 \\
\hline $2 R$ & 4168631 \\
\hline $2 R$ & 4170063 \\
\hline $2 \mathrm{R}$ & 4170400 \\
\hline $2 \mathrm{R}$ & 4170571 \\
\hline $2 \mathrm{R}$ & 4172682 \\
\hline $2 R$ & 4173193 \\
\hline $2 \mathrm{R}$ & 4173770 \\
\hline $2 \mathrm{R}$ & 4173842 \\
\hline $2 \mathrm{R}$ & 4174889 \\
\hline $2 \mathrm{R}$ & 4175715 \\
\hline $2 \mathrm{R}$ & 4179139 \\
\hline $2 \mathrm{R}$ & 4182470 \\
\hline $2 R$ & 4182667 \\
\hline $2 \mathrm{R}$ & 4183215 \\
\hline $2 \mathrm{R}$ & 4184462 \\
\hline $2 \mathrm{R}$ & 4185763 \\
\hline $2 \mathrm{R}$ & 4186222 \\
\hline $2 R$ & 4186688 \\
\hline $2 \mathrm{R}$ & 4188324 \\
\hline $2 \mathrm{R}$ & 4188735 \\
\hline $2 \mathrm{R}$ & 4190686 \\
\hline $2 \mathrm{R}$ & 4191653 \\
\hline $2 \mathrm{R}$ & 4195164 \\
\hline $2 \mathrm{R}$ & 4195690 \\
\hline $2 R$ & 4197605 \\
\hline $2 R$ & 4199104 \\
\hline $2 \mathrm{R}$ & 4199743 \\
\hline $2 \mathrm{R}$ & 4200562 \\
\hline $2 \mathrm{R}$ & 4203868 \\
\hline $2 \mathrm{R}$ & 4203938 \\
\hline $2 R$ & 4204995 \\
\hline $2 \mathrm{R}$ & 4205387 \\
\hline $2 \mathrm{R}$ & 4205442 \\
\hline $2 \mathrm{R}$ & 4205470 \\
\hline $2 \mathrm{R}$ & 4207953 \\
\hline $2 \mathrm{R}$ & 4208733 \\
\hline $2 \mathrm{R}$ & 4208830 \\
\hline $2 R$ & 4210279 \\
\hline $2 \mathrm{R}$ & 4211023 \\
\hline $2 \mathrm{R}$ & 4211314 \\
\hline $2 \mathrm{R}$ & 4211620 \\
\hline $2 \mathrm{R}$ & 4213615 \\
\hline $2 R$ & 4214322 \\
\hline $2 R$ & 4215077 \\
\hline $2 \mathrm{R}$ & 4215389 \\
\hline $2 \mathrm{R}$ & 4215504 \\
\hline $2 \mathrm{R}$ & 4218387 \\
\hline $2 \mathrm{R}$ & 4219099 \\
\hline 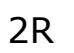 & 421 \\
\hline
\end{tabular}

\begin{tabular}{|c|c|}
\hline 2 & 5 \\
\hline 2 & 15 \\
\hline 2 & 5 \\
\hline 2 & 4 \\
\hline 2 & 18 \\
\hline 2 & 26 \\
\hline 2 & 7 \\
\hline 2 & 6 \\
\hline 2 & 5 \\
\hline 2 & 7 \\
\hline 2 & 17 \\
\hline 2 & 4 \\
\hline 2 & 23 \\
\hline 2 & 4 \\
\hline 2 & 13 \\
\hline 2 & 10 \\
\hline 2 & 6 \\
\hline 2 & 15 \\
\hline 2 & 14 \\
\hline 2 & 10 \\
\hline 2 & 18 \\
\hline 2 & 11 \\
\hline 2 & 17 \\
\hline 2 & 15 \\
\hline 2 & 10 \\
\hline 2 & 5 \\
\hline 2 & 9 \\
\hline 2 & 20 \\
\hline 2 & 23 \\
\hline 2 & 26 \\
\hline 2 & 20 \\
\hline 2 & 15 \\
\hline 2 & 11 \\
\hline 2 & 12 \\
\hline 2 & 9 \\
\hline 2 & 21 \\
\hline 2 & 28 \\
\hline 2 & 21 \\
\hline 2 & 16 \\
\hline 2 & 13 \\
\hline 2 & 20 \\
\hline 2 & 17 \\
\hline 2 & 6 \\
\hline 2 & 10 \\
\hline 2 & 18 \\
\hline 2 & 17 \\
\hline 2 & 17 \\
\hline 2 & 13 \\
\hline 2 & 38 \\
\hline 2 & 17 \\
\hline 2 & 5 \\
\hline 2 & 10 \\
\hline 2 & \\
\hline
\end{tabular}

19

21

12

15

4

14

6

$$
7
$$

17

17

15

11

16

20

4

13

7

13

7

4
14

5

12

5

9

12

8

16

8

20

8

6

7

6

17

11

10

4

7

9

10

8

10

7

4

10

4

17

7

16

8

7

6
0

0

0

0

0

0

0

0

0

0

0

0

0

0

0

0

0

0

0

0

0

0

0

0

0

0

0

0

0

0

0

0

0

0

0

0

0

0

0

0

0

0

0

0

0

0

0

0

0 


\begin{tabular}{|c|c|}
\hline $2 \mathrm{R}$ & 4220930 \\
\hline $2 \mathrm{R}$ & 4221453 \\
\hline $2 \mathrm{R}$ & 4221753 \\
\hline $2 \mathrm{R}$ & 4222886 \\
\hline $2 R$ & 4223461 \\
\hline $2 R$ & 4223523 \\
\hline $2 \mathrm{R}$ & 4224092 \\
\hline $2 R$ & 4224767 \\
\hline $2 \mathrm{R}$ & 4225282 \\
\hline $2 R$ & 4225544 \\
\hline $2 \mathrm{R}$ & 4226815 \\
\hline $2 \mathrm{R}$ & 4227321 \\
\hline $2 \mathrm{R}$ & 4232365 \\
\hline $2 \mathrm{R}$ & 4234161 \\
\hline $2 \mathrm{R}$ & 4234588 \\
\hline $2 R$ & 4234703 \\
\hline $2 R$ & 4234736 \\
\hline $2 R$ & 4235896 \\
\hline $2 \mathrm{R}$ & 4236027 \\
\hline $2 \mathrm{R}$ & 4236095 \\
\hline $2 \mathrm{R}$ & 4237670 \\
\hline $2 \mathrm{R}$ & 4237726 \\
\hline $2 \mathrm{R}$ & 4239446 \\
\hline $2 \mathrm{R}$ & 4244308 \\
\hline $2 \mathrm{R}$ & 4244350 \\
\hline $2 \mathrm{R}$ & 4244633 \\
\hline $2 \mathrm{R}$ & 4245566 \\
\hline $2 \mathrm{R}$ & 4245598 \\
\hline $2 R$ & 4246799 \\
\hline $2 \mathrm{R}$ & 4246951 \\
\hline $2 \mathrm{R}$ & 4247063 \\
\hline $2 \mathrm{R}$ & 4248042 \\
\hline $2 \mathrm{R}$ & 4249339 \\
\hline $2 \mathrm{R}$ & 4249551 \\
\hline $2 R$ & 4249951 \\
\hline $2 \mathrm{R}$ & 4251702 \\
\hline $2 \mathrm{R}$ & 4251918 \\
\hline $2 \mathrm{R}$ & 4252458 \\
\hline $2 \mathrm{R}$ & 4252645 \\
\hline $2 \mathrm{R}$ & 4255996 \\
\hline $2 \mathrm{R}$ & 4257639 \\
\hline $2 R$ & 4258194 \\
\hline $2 \mathrm{R}$ & 4259712 \\
\hline $2 R$ & 4259964 \\
\hline $2 \mathrm{R}$ & 4260531 \\
\hline $2 \mathrm{R}$ & 4261270 \\
\hline $2 \mathrm{R}$ & 4261322 \\
\hline $2 \mathrm{R}$ & 4262914 \\
\hline $2 \mathrm{R}$ & 4263967 \\
\hline $2 \mathrm{R}$ & 4266435 \\
\hline $2 \mathrm{R}$ & 4266933 \\
\hline $2 \mathrm{R}$ & 4267339 \\
\hline 21 & 426834 \\
\hline
\end{tabular}

2

2

2

2

2

2

2

2

2

2

2

2

2

2

2

2

2

2

2

2

2

2

2

2

2

2

2

2

2

2

2

2

2

2

2

2

2

2

2

2

2

2

2

2

2

2

2

2

2

2

2

2

2
17

5

4

16

8

14

28

27

22

13

24

20

15

18

12

21

15

11

8

26

8

7

16

6

8

18

7

20

22

7

27

22

18

22

6

17

11

20

16

26

9

18

13

11

13

7

14

26

7

11

16

6

15
17

13

13

14

10

5

8

12

14

5

9

14

9

13

11

11

7

5

12

14

5

25

4

8

9

12

16

10

4

7

17

8

23

9

5

5

19

12

12

12

7

6

4

28

4

7

5

15

19

12

9

19

9
0

0

0

0

0

0

0

0

0

0

0

0

0

0

0

0

0

0

0

0

0

0

0

0

0

0

0

0

0

0

0

0

0

0

0

0

0

0

0

0

0
0

0

0

0

0

0

0 
$2 \mathrm{R}$

$2 R$

$2 R$

$2 R$

$2 R$

$2 R$

$2 \mathrm{R}$

$2 \mathrm{R}$

$2 \mathrm{R}$

$2 \mathrm{R}$

$2 \mathrm{R}$

$2 \mathrm{R}$

$2 \mathrm{R}$

$2 \mathrm{R}$

$2 \mathrm{R}$

$2 \mathrm{R}$

$2 \mathrm{R}$

$2 \mathrm{R}$

$2 \mathrm{R}$

$2 \mathrm{R}$

$2 \mathrm{R}$

$2 \mathrm{R}$

$2 \mathrm{R}$

$2 \mathrm{R}$

$2 \mathrm{R}$

$2 \mathrm{R}$

$2 \mathrm{R}$

$2 \mathrm{R}$

$2 \mathrm{R}$

$2 \mathrm{R}$

$2 \mathrm{R}$

$2 \mathrm{R}$

$2 \mathrm{R}$

$2 \mathrm{R}$

$2 \mathrm{R}$

$2 \mathrm{R}$

$2 \mathrm{R}$

$2 \mathrm{R}$

$2 \mathrm{R}$

$2 \mathrm{R}$

$2 \mathrm{R}$

$2 \mathrm{R}$

$2 \mathrm{R}$

$2 \mathrm{R}$

$2 \mathrm{R}$

$2 \mathrm{R}$

$2 \mathrm{R}$

$2 \mathrm{R}$

$2 \mathrm{R}$

$2 \mathrm{R}$

$2 \mathrm{R}$

$2 \mathrm{R}$

$2 \mathrm{R}$
4268661

4268903

4269378

4270499

4270664

4271121

4272000

4272895

4274188

4274586

4275982

4276047

4276249

4277752

4278507

4278916

4278994

4279470

4280298

4280555

4282905

4283460

4291213

4291249

4291588

4291670

4291904

4291983

4292157

4292859

4294068

4294782

4295031

4295083

4299950

4300847

4301052

4303514

4303672

4304901

4313631

4313669

4315669

4315698

4318646

4318938

4320603

4320861

4321121

4326644

4329032

4329217

4329647
2

2

2

2

2

2

2

2

2

2

2

2

2

2

2

2

2

2

2

2

2

2

2

2

2

2

2

2

2

2

2

2

2

2

2

2

2

2

2

2

2

2

2

2

2

2

2

2

2

2

2

2

2
16

14

19

13

8

10

15

7

6

17

10

10

25

15

10

13

30

15

5

13

15

5

6

20

8

7

16

23

6

19

11

5

13

5

12

25

15

25

12

14

14

9

19

24

14

10

11

24

14

15

9

8

6

$\begin{array}{rl}9 & 0 \\ 13 & 0 \\ 12 & 0 \\ 13 & 0 \\ 21 & 0 \\ 4 & 0 \\ 16 & 0 \\ 6 & 0 \\ 9 & 0 \\ 6 & 0 \\ 9 & 0 \\ 6 & 0 \\ 15 & 0 \\ 7 & 0 \\ 5 & 0 \\ 9 & 0 \\ 12 & 0 \\ 7 & 0 \\ 13 & 0 \\ 4 & 0 \\ 10 & 0\end{array}$

$10 \quad 0$

110

35

18

7

23

8

18

17

5

6

7

8

6

22

22

4

6

7

12

7

6

19

15

8

5

4

21

8

16

8

12

14

0
0
0
0
0
0
0
0
0
0
0
0
0
0
0
0
0
0
0
0
0
0
0

0

0

0

0

0

0

0

0

0

0

0

0

0

0

0

0

0

0

0

0

0

0

0

0

0
0

0 


\begin{tabular}{|c|c|c|c|c|c|}
\hline $2 \mathrm{R}$ & 4338772 & 2 & 18 & 17 & 0 \\
\hline $2 \mathrm{R}$ & 4356187 & 2 & 4 & 26 & 0 \\
\hline $2 \mathrm{R}$ & 4357856 & 2 & 20 & 20 & 0 \\
\hline $2 \mathrm{R}$ & 4358018 & 2 & 21 & 4 & 0 \\
\hline $2 R$ & 4358664 & 2 & 20 & 15 & 0 \\
\hline $2 \mathrm{R}$ & 4358729 & 2 & 24 & 4 & 0 \\
\hline $2 \mathrm{R}$ & 4358816 & 2 & 21 & 14 & 0 \\
\hline $2 \mathrm{R}$ & 4358944 & 2 & 15 & 16 & 0 \\
\hline $2 \mathrm{R}$ & 4359074 & 2 & 18 & 27 & 0 \\
\hline $2 R$ & 4360693 & 2 & 10 & 25 & 0 \\
\hline $2 \mathrm{R}$ & 4360804 & 2 & 6 & 15 & 0 \\
\hline $2 \mathrm{R}$ & 4360921 & 2 & 13 & 24 & 0 \\
\hline $2 \mathrm{R}$ & 4365120 & 2 & 4 & 16 & 0 \\
\hline $2 \mathrm{R}$ & 4365241 & 2 & 7 & 8 & 0 \\
\hline $2 \mathrm{R}$ & 4365765 & 2 & 5 & 10 & 0 \\
\hline $2 \mathrm{R}$ & 4367758 & 2 & 17 & 5 & 0 \\
\hline $2 \mathrm{R}$ & 4368084 & 2 & 28 & 16 & 0 \\
\hline $2 \mathrm{R}$ & 4368756 & 2 & 17 & 7 & 0 \\
\hline $2 \mathrm{R}$ & 4369748 & 2 & 17 & 9 & 0 \\
\hline $2 R$ & 4369865 & 2 & 30 & 13 & 0 \\
\hline $2 \mathrm{R}$ & 4370507 & 2 & 27 & 13 & 0 \\
\hline $2 \mathrm{R}$ & 4374658 & 2 & 6 & 7 & 0 \\
\hline $2 \mathrm{R}$ & 4374847 & 2 & 22 & 9 & 0 \\
\hline $2 R$ & 4376121 & 2 & 19 & 6 & 0 \\
\hline $2 \mathrm{R}$ & 4376417 & 2 & 10 & 26 & 0 \\
\hline $2 \mathrm{R}$ & 4376604 & 2 & 5 & 5 & 0 \\
\hline $2 \mathrm{R}$ & 4377077 & 2 & 8 & 6 & 0 \\
\hline $2 \mathrm{R}$ & 4381024 & 2 & 13 & 4 & 0 \\
\hline $2 R$ & 4382219 & 2 & 12 & 5 & 0 \\
\hline $2 \mathrm{R}$ & 4383623 & 2 & 10 & 15 & 0 \\
\hline $2 \mathrm{R}$ & 4386050 & 2 & 18 & 5 & 0 \\
\hline $2 R$ & 4387334 & 2 & 8 & 10 & 0 \\
\hline $2 \mathrm{R}$ & 4389781 & 2 & 20 & 8 & 0 \\
\hline $2 \mathrm{R}$ & 4389884 & 2 & 14 & 10 & 0 \\
\hline $2 R$ & 4389948 & 2 & 28 & 15 & 0 \\
\hline $2 \mathrm{R}$ & 4390166 & 2 & 10 & 4 & 0 \\
\hline $2 \mathrm{R}$ & 4390239 & 2 & 27 & 7 & 0 \\
\hline $2 \mathrm{R}$ & 4392165 & 2 & 16 & 4 & 0 \\
\hline $2 \mathrm{R}$ & 4397170 & 2 & 9 & 10 & 0 \\
\hline $2 \mathrm{R}$ & 4397362 & 2 & 4 & 9 & 0 \\
\hline $2 R$ & 4397978 & 2 & 24 & 13 & 0 \\
\hline $2 \mathrm{R}$ & 4398644 & 2 & 7 & 6 & 0 \\
\hline $2 \mathrm{R}$ & 4398703 & 2 & 6 & 19 & 0 \\
\hline $2 R$ & 4398850 & 2 & 6 & 11 & 0 \\
\hline $2 \mathrm{R}$ & 4399028 & 2 & 7 & 25 & 0 \\
\hline $2 \mathrm{R}$ & 4399970 & 2 & 12 & 15 & 0 \\
\hline $2 R$ & 4402029 & 2 & 11 & 5 & 0 \\
\hline $2 R$ & 4402272 & 2 & 7 & 8 & 0 \\
\hline $2 \mathrm{R}$ & 4402631 & 2 & 14 & 6 & 0 \\
\hline $2 R$ & 4402761 & 2 & 28 & 5 & 0 \\
\hline $2 \mathrm{R}$ & 4403000 & 2 & 17 & 12 & 0 \\
\hline $2 \mathrm{R}$ & 4403863 & 2 & 5 & 5 & 0 \\
\hline $2 R$ & 4403963 & 2 & 16 & 12 & 0 \\
\hline
\end{tabular}




\begin{tabular}{|c|c|c|c|}
\hline $2 \mathrm{R}$ & 4405604 & 2 & 5 \\
\hline $2 \mathrm{R}$ & 4405668 & 2 & 15 \\
\hline $2 \mathrm{R}$ & 4405904 & 2 & 10 \\
\hline $2 \mathrm{R}$ & 4405979 & 2 & 9 \\
\hline $2 R$ & 4409390 & 2 & 30 \\
\hline $2 \mathrm{R}$ & 4409473 & 2 & 28 \\
\hline $2 \mathrm{R}$ & 4410332 & 2 & 11 \\
\hline $2 \mathrm{R}$ & 4410877 & 2 & 17 \\
\hline $2 \mathrm{R}$ & 4413067 & 2 & 16 \\
\hline $2 \mathrm{R}$ & 4413562 & 2 & 10 \\
\hline $2 \mathrm{R}$ & 4415201 & 2 & 25 \\
\hline $2 \mathrm{R}$ & 4416203 & 2 & 20 \\
\hline $2 \mathrm{R}$ & 4417899 & 2 & 14 \\
\hline $2 \mathrm{R}$ & 4418458 & 2 & 16 \\
\hline $2 \mathrm{R}$ & 4419448 & 2 & 4 \\
\hline $2 \mathrm{R}$ & 4421098 & 2 & 10 \\
\hline $2 \mathrm{R}$ & 4421475 & 2 & 20 \\
\hline $2 \mathrm{R}$ & 4422261 & 2 & 7 \\
\hline $2 \mathrm{R}$ & 4422560 & 2 & 9 \\
\hline $2 \mathrm{R}$ & 4423288 & 2 & 5 \\
\hline $2 \mathrm{R}$ & 4423402 & 2 & 12 \\
\hline $2 \mathrm{R}$ & 4424124 & 2 & 22 \\
\hline $2 \mathrm{R}$ & 4424188 & 2 & 14 \\
\hline $2 \mathrm{R}$ & 4424300 & 2 & 15 \\
\hline $2 \mathrm{R}$ & 4424402 & 2 & 5 \\
\hline $2 \mathrm{R}$ & 4425054 & 2 & 9 \\
\hline $2 \mathrm{R}$ & 4426412 & 2 & 5 \\
\hline $2 \mathrm{R}$ & 4427009 & 2 & 4 \\
\hline $2 \mathrm{R}$ & 4427867 & 2 & 17 \\
\hline $2 \mathrm{R}$ & 4428794 & 2 & 5 \\
\hline $2 \mathrm{R}$ & 4429171 & 2 & 6 \\
\hline $2 R$ & 4429346 & 2 & 11 \\
\hline $2 \mathrm{R}$ & 4430567 & 2 & 7 \\
\hline $2 \mathrm{R}$ & 4432041 & 2 & 13 \\
\hline $2 R$ & 4432149 & 2 & 7 \\
\hline $2 \mathrm{R}$ & 4432245 & 2 & 4 \\
\hline $2 \mathrm{R}$ & 4433400 & 2 & 28 \\
\hline $2 \mathrm{R}$ & 4434415 & 2 & 7 \\
\hline $2 \mathrm{R}$ & 4434552 & 2 & 8 \\
\hline $2 \mathrm{R}$ & 4435101 & 2 & 13 \\
\hline $2 \mathrm{R}$ & 4435231 & 2 & 14 \\
\hline $2 \mathrm{R}$ & 4437053 & 2 & 18 \\
\hline $2 \mathrm{R}$ & 4437978 & 2 & 11 \\
\hline $2 \mathrm{R}$ & 4439624 & 2 & 24 \\
\hline $2 \mathrm{R}$ & 4440610 & 2 & 19 \\
\hline $2 \mathrm{R}$ & 4441184 & 2 & 4 \\
\hline $2 \mathrm{R}$ & 4441246 & 2 & 14 \\
\hline $2 R$ & 4441649 & 2 & 5 \\
\hline $2 \mathrm{R}$ & 4441762 & 2 & 14 \\
\hline $2 \mathrm{R}$ & 4441816 & 2 & 15 \\
\hline $2 \mathrm{R}$ & 4442979 & 2 & 10 \\
\hline $2 \mathrm{R}$ & 4443156 & 2 & 19 \\
\hline $2 \mathrm{R}$ & 4443420 & 2 & 7 \\
\hline
\end{tabular}

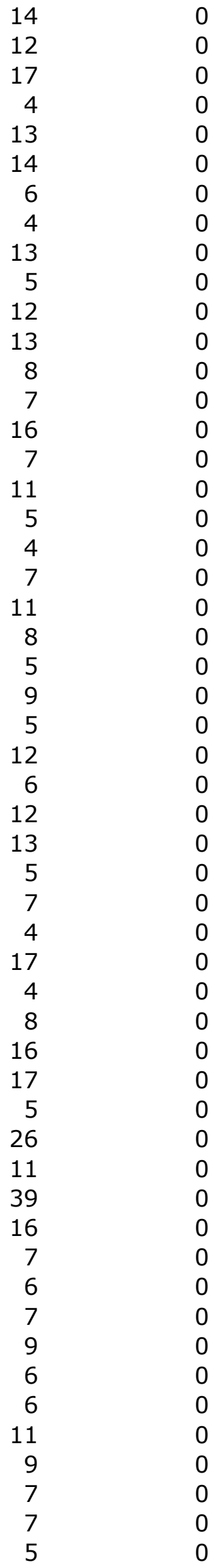




\begin{tabular}{|c|c|c|c|}
\hline $2 \mathrm{R}$ & 4444608 & 2 & 19 \\
\hline $2 \mathrm{R}$ & 4445736 & 2 & 16 \\
\hline $2 \mathrm{R}$ & 4445942 & 2 & 7 \\
\hline $2 \mathrm{R}$ & 4447049 & 2 & 15 \\
\hline $2 \mathrm{R}$ & 4447366 & 2 & 11 \\
\hline $2 \mathrm{R}$ & 4447463 & 2 & 34 \\
\hline $2 \mathrm{R}$ & 4447545 & 2 & 5 \\
\hline $2 \mathrm{R}$ & 4448556 & 2 & 37 \\
\hline $2 \mathrm{R}$ & 4448624 & 2 & 18 \\
\hline $2 \mathrm{R}$ & 4449350 & 2 & 5 \\
\hline $2 \mathrm{R}$ & 4449601 & 2 & 15 \\
\hline $2 \mathrm{R}$ & 4450353 & 2 & 12 \\
\hline $2 \mathrm{R}$ & 4450588 & 2 & 22 \\
\hline $2 \mathrm{R}$ & 4452794 & 2 & 24 \\
\hline $2 \mathrm{R}$ & 4454918 & 2 & 5 \\
\hline $2 \mathrm{R}$ & 4455744 & 2 & 16 \\
\hline $2 \mathrm{R}$ & 4455772 & 2 & 12 \\
\hline $2 \mathrm{R}$ & 4457383 & 2 & 23 \\
\hline $2 \mathrm{R}$ & 4458819 & 2 & 20 \\
\hline $2 \mathrm{R}$ & 4459026 & 2 & 23 \\
\hline $2 \mathrm{R}$ & 4459463 & 2 & 22 \\
\hline $2 \mathrm{R}$ & 4461012 & 2 & 14 \\
\hline $2 \mathrm{R}$ & 4462673 & 2 & 7 \\
\hline $2 \mathrm{R}$ & 4463292 & 2 & 12 \\
\hline $2 \mathrm{R}$ & 4464789 & 2 & 25 \\
\hline $2 \mathrm{R}$ & 4465936 & 2 & 15 \\
\hline $2 \mathrm{R}$ & 4466099 & 2 & 9 \\
\hline $2 \mathrm{R}$ & 4466406 & 2 & 9 \\
\hline $2 \mathrm{R}$ & 4466790 & 2 & 4 \\
\hline $2 \mathrm{R}$ & 4467087 & 2 & 13 \\
\hline $2 \mathrm{R}$ & 4467244 & 2 & 19 \\
\hline $2 \mathrm{R}$ & 4468329 & 2 & 24 \\
\hline $2 \mathrm{R}$ & 4468681 & 2 & 14 \\
\hline $2 \mathrm{R}$ & 4469108 & 2 & 15 \\
\hline $2 \mathrm{R}$ & 4469156 & 2 & 11 \\
\hline $2 \mathrm{R}$ & 4469848 & 2 & 9 \\
\hline $2 \mathrm{R}$ & 4469878 & 2 & 10 \\
\hline $2 \mathrm{R}$ & 4470182 & 2 & 19 \\
\hline $2 \mathrm{R}$ & 4471074 & 2 & 11 \\
\hline $2 \mathrm{R}$ & 4473215 & 2 & 13 \\
\hline $2 \mathrm{R}$ & 4473388 & 2 & 16 \\
\hline $2 \mathrm{R}$ & 4473478 & 2 & 11 \\
\hline $2 \mathrm{R}$ & 4474082 & 2 & 10 \\
\hline $2 \mathrm{R}$ & 4474367 & 2 & 12 \\
\hline $2 \mathrm{R}$ & 4474715 & 2 & 13 \\
\hline $2 \mathrm{R}$ & 4475140 & 2 & 17 \\
\hline $2 \mathrm{R}$ & 4475411 & 2 & 8 \\
\hline $2 \mathrm{R}$ & 4476017 & 2 & 29 \\
\hline $2 \mathrm{R}$ & 4476822 & 2 & 10 \\
\hline $2 \mathrm{R}$ & 4476988 & 2 & 15 \\
\hline $2 \mathrm{R}$ & 4477423 & 2 & 24 \\
\hline $2 \mathrm{R}$ & 4478034 & 2 & 17 \\
\hline $2 R$ & 4478961 & 2 & 7 \\
\hline
\end{tabular}

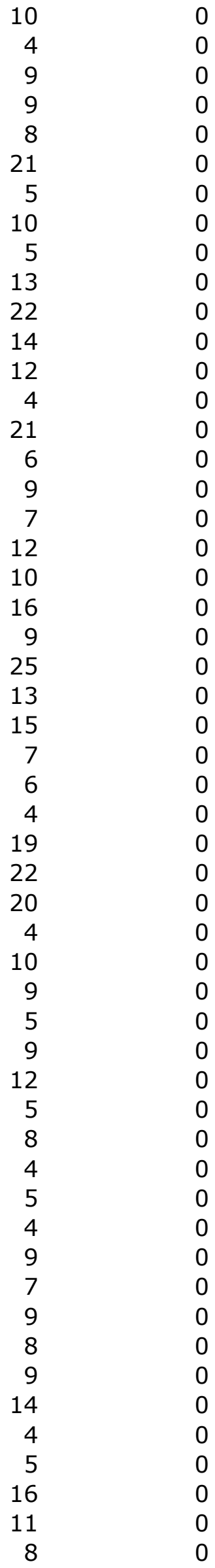




\begin{tabular}{|c|c|c|c|c|c|}
\hline $2 \mathrm{R}$ & 4479939 & 2 & 12 & 4 & 0 \\
\hline $2 \mathrm{R}$ & 4480327 & 2 & 15 & 11 & 0 \\
\hline $2 \mathrm{R}$ & 4482057 & 2 & 9 & 6 & 0 \\
\hline $2 \mathrm{R}$ & 4482332 & 2 & 26 & 8 & 0 \\
\hline $2 \mathrm{R}$ & 4482835 & 2 & 21 & 11 & 0 \\
\hline $2 \mathrm{R}$ & 4483796 & 2 & 15 & 6 & 0 \\
\hline $2 \mathrm{R}$ & 4485560 & 2 & 9 & 23 & 0 \\
\hline $2 \mathrm{R}$ & 4486918 & 2 & 7 & 13 & 0 \\
\hline $2 \mathrm{R}$ & 4488793 & 2 & 9 & 18 & 0 \\
\hline $2 \mathrm{R}$ & 4489118 & 2 & 12 & 5 & 0 \\
\hline $2 \mathrm{R}$ & 4489949 & 2 & 16 & 28 & 0 \\
\hline $2 R$ & 4490392 & 2 & 5 & 20 & 0 \\
\hline $2 R$ & 4491725 & 2 & 24 & 20 & 0 \\
\hline $2 \mathrm{R}$ & 4492048 & 2 & 18 & 11 & 0 \\
\hline $2 R$ & 4492536 & 2 & 18 & 13 & 0 \\
\hline $2 \mathrm{R}$ & 4493444 & 2 & 8 & 5 & 0 \\
\hline $2 \mathrm{R}$ & 4494466 & 2 & 21 & 10 & 0 \\
\hline $2 \mathrm{R}$ & 4494761 & 2 & 11 & 6 & 0 \\
\hline $2 R$ & 4495689 & 2 & 23 & 7 & 0 \\
\hline $2 \mathrm{R}$ & 4496560 & 2 & 10 & 4 & 0 \\
\hline $2 \mathrm{R}$ & 4496707 & 2 & 4 & 15 & 0 \\
\hline $2 R$ & 4498120 & 2 & 9 & 4 & 0 \\
\hline $2 \mathrm{R}$ & 4498800 & 2 & 15 & 15 & 0 \\
\hline $2 R$ & 4498871 & 2 & 20 & 9 & 0 \\
\hline $2 \mathrm{R}$ & 4499274 & 2 & 4 & 15 & 0 \\
\hline $2 \mathrm{R}$ & 4499456 & 2 & 11 & 6 & 0 \\
\hline $2 \mathrm{R}$ & 4500032 & 2 & 11 & 5 & 0 \\
\hline $2 R$ & 4500268 & 2 & 4 & 17 & 0 \\
\hline $2 \mathrm{R}$ & 4500424 & 2 & 8 & 13 & 0 \\
\hline $2 \mathrm{R}$ & 4501379 & 2 & 22 & 9 & 0 \\
\hline $2 R$ & 4501840 & 2 & 13 & 7 & 0 \\
\hline $2 \mathrm{R}$ & 4502911 & 2 & 10 & 21 & 0 \\
\hline $2 \mathrm{R}$ & 4504543 & 2 & 5 & 6 & 0 \\
\hline $2 \mathrm{R}$ & 4505736 & 2 & 5 & 18 & 0 \\
\hline $2 \mathrm{R}$ & 4506260 & 2 & 9 & 11 & 0 \\
\hline $2 R$ & 4506551 & 2 & 31 & 14 & 0 \\
\hline $2 R$ & 4507772 & 2 & 13 & 7 & 0 \\
\hline $2 \mathrm{R}$ & 4508682 & 2 & 29 & 21 & 0 \\
\hline $2 \mathrm{R}$ & 4509076 & 2 & 18 & 22 & 0 \\
\hline $2 \mathrm{R}$ & 4511754 & 2 & 18 & 4 & 0 \\
\hline $2 \mathrm{R}$ & 4512580 & 2 & 28 & 16 & 0 \\
\hline $2 \mathrm{R}$ & 4513500 & 2 & 8 & 8 & 0 \\
\hline $2 R$ & 4513616 & 2 & 18 & 9 & 0 \\
\hline $2 \mathrm{R}$ & 4513814 & 2 & 12 & 13 & 0 \\
\hline $2 \mathrm{R}$ & 4513915 & 2 & 19 & 8 & 0 \\
\hline $2 \mathrm{R}$ & 4514328 & 2 & 20 & 10 & 0 \\
\hline $2 \mathrm{R}$ & 4514451 & 2 & 21 & 15 & 0 \\
\hline $2 \mathrm{R}$ & 4514544 & 2 & 23 & 5 & 0 \\
\hline $2 R$ & 4514607 & 2 & 32 & 7 & 0 \\
\hline $2 \mathrm{R}$ & 4514753 & 2 & 8 & 8 & 0 \\
\hline $2 \mathrm{R}$ & 4514904 & 2 & 12 & 4 & 0 \\
\hline $2 \mathrm{R}$ & 4514974 & 2 & 15 & 17 & 0 \\
\hline $2 R$ & 4515517 & 2 & 12 & 5 & 0 \\
\hline
\end{tabular}




\begin{tabular}{|c|c|c|c|c|}
\hline $2 \mathrm{R}$ & 4516141 & 2 & 15 & 5 \\
\hline $2 \mathrm{R}$ & 4516450 & 2 & 15 & 10 \\
\hline $2 \mathrm{R}$ & 4516643 & 2 & 18 & 4 \\
\hline $2 \mathrm{R}$ & 4517151 & 2 & 23 & 14 \\
\hline $2 \mathrm{R}$ & 4517636 & 2 & 24 & 9 \\
\hline $2 \mathrm{R}$ & 4518784 & 2 & 5 & 8 \\
\hline $2 \mathrm{R}$ & 4518916 & 2 & 10 & 9 \\
\hline $2 R$ & 4519324 & 2 & 30 & 21 \\
\hline $2 \mathrm{R}$ & 4519617 & 2 & 12 & 5 \\
\hline $2 \mathrm{R}$ & 4519663 & 2 & 20 & 8 \\
\hline $2 \mathrm{R}$ & 4519766 & 2 & 13 & 4 \\
\hline $2 R$ & 4519805 & 2 & 21 & 13 \\
\hline $2 \mathrm{R}$ & 4519870 & 2 & 19 & 10 \\
\hline $2 \mathrm{R}$ & 4520489 & 2 & 22 & 6 \\
\hline $2 \mathrm{R}$ & 4521369 & 2 & 14 & 6 \\
\hline $2 \mathrm{R}$ & 4521972 & 2 & 19 & 7 \\
\hline $2 \mathrm{R}$ & 4522690 & 2 & 21 & 6 \\
\hline $2 \mathrm{R}$ & 4524375 & 2 & 14 & 7 \\
\hline $2 \mathrm{R}$ & 4525356 & 2 & 11 & 7 \\
\hline $2 \mathrm{R}$ & 4525987 & 2 & 14 & 5 \\
\hline $2 \mathrm{R}$ & 4526229 & 2 & 27 & 7 \\
\hline $2 \mathrm{R}$ & 4526400 & 2 & 16 & 4 \\
\hline $2 \mathrm{R}$ & 4526508 & 2 & 7 & 24 \\
\hline $2 \mathrm{R}$ & 4526554 & 2 & 10 & 22 \\
\hline $2 \mathrm{R}$ & 4526611 & 2 & 13 & 4 \\
\hline $2 \mathrm{R}$ & 4527180 & 2 & 4 & 9 \\
\hline $2 \mathrm{R}$ & 4527836 & 2 & 12 & 4 \\
\hline $2 \mathrm{R}$ & 4528077 & 2 & 34 & 16 \\
\hline $2 \mathrm{R}$ & 4530115 & 2 & 7 & 8 \\
\hline $2 \mathrm{R}$ & 4531617 & 2 & 6 & 5 \\
\hline $2 \mathrm{R}$ & 4532231 & 2 & 5 & 15 \\
\hline $2 \mathrm{R}$ & 4534783 & 2 & 10 & 5 \\
\hline $2 \mathrm{R}$ & 4535391 & 2 & 7 & 23 \\
\hline $2 \mathrm{R}$ & 4535845 & 2 & 7 & 10 \\
\hline $2 \mathrm{R}$ & 4537537 & 2 & 15 & 8 \\
\hline $2 \mathrm{R}$ & 4537605 & 2 & 21 & 23 \\
\hline $2 \mathrm{R}$ & 4538980 & 2 & 9 & 9 \\
\hline $2 \mathrm{R}$ & 4539075 & 2 & 6 & 6 \\
\hline $2 \mathrm{R}$ & 4540734 & 2 & 19 & 7 \\
\hline $2 \mathrm{R}$ & 4541136 & 2 & 12 & 5 \\
\hline $2 \mathrm{R}$ & 4541449 & 2 & 9 & 9 \\
\hline $2 \mathrm{R}$ & 4541642 & 2 & 23 & 10 \\
\hline $2 \mathrm{R}$ & 4541689 & 2 & 19 & 11 \\
\hline $2 \mathrm{R}$ & 4547173 & 2 & 23 & 9 \\
\hline $2 \mathrm{R}$ & 4548040 & 2 & 9 & 17 \\
\hline $2 \mathrm{R}$ & 4548813 & 2 & 4 & 9 \\
\hline $2 \mathrm{R}$ & 4549695 & 2 & 5 & 21 \\
\hline $2 \mathrm{R}$ & 4549769 & 2 & 11 & 25 \\
\hline $2 \mathrm{R}$ & 4551268 & 2 & 19 & 7 \\
\hline $2 \mathrm{R}$ & 4552498 & 2 & 15 & 5 \\
\hline $2 \mathrm{R}$ & 4553865 & 2 & 9 & 7 \\
\hline $2 \mathrm{R}$ & 4554060 & 2 & 13 & 12 \\
\hline $2 \mathrm{R}$ & 4554716 & 2 & 18 & 22 \\
\hline
\end{tabular}




\begin{tabular}{|c|c|c|c|c|c|}
\hline $2 \mathrm{R}$ & 4554948 & 2 & 11 & 8 & 0 \\
\hline $2 \mathrm{R}$ & 4555046 & 2 & 20 & 4 & 0 \\
\hline $2 \mathrm{R}$ & 4555392 & 2 & 21 & 9 & 0 \\
\hline $2 \mathrm{R}$ & 4555931 & 2 & 12 & 4 & 0 \\
\hline $2 R$ & 4556063 & 2 & 19 & 13 & 0 \\
\hline $2 \mathrm{R}$ & 4556284 & 2 & 9 & 9 & 0 \\
\hline $2 \mathrm{R}$ & 4556399 & 2 & 14 & 10 & 0 \\
\hline $2 \mathrm{R}$ & 4556477 & 2 & 9 & 13 & 0 \\
\hline $2 \mathrm{R}$ & 4556679 & 2 & 12 & 8 & 0 \\
\hline $2 R$ & 4561209 & 2 & 4 & 16 & 0 \\
\hline $2 \mathrm{R}$ & 4561223 & 2 & 4 & 12 & 0 \\
\hline $2 \mathrm{R}$ & 4561244 & 2 & 11 & 10 & 0 \\
\hline $2 \mathrm{R}$ & 4562020 & 2 & 26 & 13 & 0 \\
\hline $2 \mathrm{R}$ & 4563016 & 2 & 14 & 9 & 0 \\
\hline $2 \mathrm{R}$ & 4563903 & 2 & 10 & 8 & 0 \\
\hline $2 \mathrm{R}$ & 4564402 & 2 & 23 & 16 & 0 \\
\hline $2 \mathrm{R}$ & 4564849 & 2 & 11 & 13 & 0 \\
\hline $2 \mathrm{R}$ & 4564857 & 2 & 9 & 13 & 0 \\
\hline $2 \mathrm{R}$ & 4565364 & 2 & 6 & 6 & 0 \\
\hline $2 \mathrm{R}$ & 4565482 & 2 & 19 & 17 & 0 \\
\hline $2 \mathrm{R}$ & 4565547 & 2 & 6 & 10 & 0 \\
\hline $2 \mathrm{R}$ & 4565952 & 2 & 17 & 8 & 0 \\
\hline $2 R$ & 4566336 & 2 & 17 & 6 & 0 \\
\hline $2 \mathrm{R}$ & 4566403 & 2 & 24 & 15 & 0 \\
\hline $2 \mathrm{R}$ & 4566458 & 2 & 23 & 21 & 0 \\
\hline $2 \mathrm{R}$ & 4566836 & 2 & 12 & 5 & 0 \\
\hline $2 \mathrm{R}$ & 4567125 & 2 & 18 & 12 & 0 \\
\hline $2 \mathrm{R}$ & 4567989 & 2 & 7 & 14 & 0 \\
\hline $2 R$ & 4568694 & 2 & 26 & 23 & 0 \\
\hline $2 \mathrm{R}$ & 4568757 & 2 & 19 & 9 & 0 \\
\hline $2 \mathrm{R}$ & 4571260 & 2 & 6 & 13 & 0 \\
\hline $2 R$ & 4571770 & 2 & 19 & 5 & 0 \\
\hline $2 \mathrm{R}$ & 4571912 & 2 & 16 & 13 & 0 \\
\hline $2 \mathrm{R}$ & 4572342 & 2 & 5 & 6 & 0 \\
\hline $2 R$ & 4572597 & 2 & 10 & 4 & 0 \\
\hline $2 \mathrm{R}$ & 4572726 & 2 & 12 & 5 & 0 \\
\hline $2 \mathrm{R}$ & 4572744 & 2 & 15 & 4 & 0 \\
\hline $2 \mathrm{R}$ & 4573764 & 2 & 12 & 10 & 0 \\
\hline $2 \mathrm{R}$ & 4573997 & 2 & 15 & 4 & 0 \\
\hline $2 \mathrm{R}$ & 4574721 & 2 & 26 & 14 & 0 \\
\hline $2 R$ & 4575995 & 2 & 14 & 4 & 0 \\
\hline $2 R$ & 4576261 & 2 & 16 & 6 & 0 \\
\hline $2 \mathrm{R}$ & 4579896 & 2 & 24 & 4 & 0 \\
\hline $2 R$ & 4579910 & 2 & 21 & 6 & 0 \\
\hline $2 \mathrm{R}$ & 4580509 & 2 & 21 & 15 & 0 \\
\hline $2 \mathrm{R}$ & 4582865 & 2 & 19 & 8 & 0 \\
\hline $2 R$ & 4583351 & 2 & 30 & 4 & 0 \\
\hline $2 R$ & 4583439 & 2 & 15 & 12 & 0 \\
\hline $2 \mathrm{R}$ & 4584729 & 2 & 25 & 7 & 0 \\
\hline $2 R$ & 4585667 & 2 & 24 & 10 & 0 \\
\hline $2 \mathrm{R}$ & 4585781 & 2 & 13 & 7 & 0 \\
\hline $2 \mathrm{R}$ & 4586026 & 2 & 20 & 9 & 0 \\
\hline $2 R$ & 4586949 & 2 & 12 & 6 & 0 \\
\hline
\end{tabular}




\begin{tabular}{|c|c|c|c|c|c|}
\hline $2 \mathrm{R}$ & 4588768 & 2 & 23 & 7 & 0 \\
\hline $2 \mathrm{R}$ & 4588861 & 2 & 15 & 6 & 0 \\
\hline $2 \mathrm{R}$ & 4589005 & 2 & 23 & 7 & 0 \\
\hline $2 \mathrm{R}$ & 4589635 & 2 & 30 & 10 & 0 \\
\hline $2 \mathrm{R}$ & 4591107 & 2 & 16 & 7 & 0 \\
\hline $2 \mathrm{R}$ & 4595176 & 2 & 24 & 6 & 0 \\
\hline $2 \mathrm{R}$ & 4595697 & 2 & 24 & 4 & 0 \\
\hline $2 \mathrm{R}$ & 4596778 & 2 & 12 & 10 & 0 \\
\hline $2 \mathrm{R}$ & 4596972 & 2 & 20 & 4 & 0 \\
\hline $2 \mathrm{R}$ & 4597969 & 2 & 15 & 10 & 0 \\
\hline $2 R$ & 4599586 & 2 & 23 & 12 & 0 \\
\hline $2 R$ & 4599762 & 2 & 14 & 17 & 0 \\
\hline $2 R$ & 4600037 & 2 & 26 & 16 & 0 \\
\hline $2 \mathrm{R}$ & 4600348 & 2 & 15 & 10 & 0 \\
\hline $2 \mathrm{R}$ & 4600368 & 2 & 9 & 13 & 0 \\
\hline $2 \mathrm{R}$ & 4600547 & 2 & 6 & 10 & 0 \\
\hline $2 \mathrm{R}$ & 4601637 & 2 & 11 & 10 & 0 \\
\hline $2 \mathrm{R}$ & 4603063 & 2 & 14 & 5 & 0 \\
\hline $2 R$ & 4603537 & 2 & 21 & 6 & 0 \\
\hline $2 \mathrm{R}$ & 4603878 & 2 & 10 & 7 & 0 \\
\hline $2 \mathrm{R}$ & 4604173 & 2 & 14 & 8 & 0 \\
\hline $2 R$ & 4604184 & 2 & 16 & 10 & 0 \\
\hline $2 \mathrm{R}$ & 4604541 & 2 & 11 & 16 & 0 \\
\hline $2 R$ & 4609188 & 2 & 17 & 15 & 0 \\
\hline $2 \mathrm{R}$ & 4611189 & 2 & 12 & 5 & 0 \\
\hline $2 \mathrm{R}$ & 4614055 & 2 & 9 & 4 & 0 \\
\hline $2 \mathrm{R}$ & 4619937 & 2 & 17 & 5 & 0 \\
\hline $2 R$ & 4620784 & 2 & 14 & 5 & 0 \\
\hline $2 \mathrm{R}$ & 4624018 & 2 & 10 & 13 & 0 \\
\hline $2 \mathrm{R}$ & 4624246 & 2 & 18 & 5 & 0 \\
\hline $2 R$ & 4624435 & 2 & 5 & 6 & 0 \\
\hline $2 \mathrm{R}$ & 4624689 & 2 & 16 & 7 & 0 \\
\hline $2 \mathrm{R}$ & 4624810 & 2 & 24 & 16 & 0 \\
\hline $2 \mathrm{R}$ & 4626449 & 2 & 5 & 16 & 0 \\
\hline $2 \mathrm{R}$ & 4626598 & 2 & 5 & 9 & 0 \\
\hline $2 R$ & 4627460 & 2 & 5 & 27 & 0 \\
\hline $2 R$ & 4628923 & 2 & 21 & 15 & 0 \\
\hline $2 \mathrm{R}$ & 4629251 & 2 & 28 & 16 & 0 \\
\hline $2 \mathrm{R}$ & 4631880 & 2 & 12 & 9 & 0 \\
\hline $2 \mathrm{R}$ & 4632562 & 2 & 4 & 11 & 0 \\
\hline $2 \mathrm{R}$ & 4633675 & 2 & 5 & 15 & 0 \\
\hline $2 \mathrm{R}$ & 4637476 & 2 & 9 & 5 & 0 \\
\hline $2 R$ & 4646288 & 2 & 10 & 6 & 0 \\
\hline $2 \mathrm{R}$ & 4646330 & 2 & 25 & 23 & 0 \\
\hline $2 \mathrm{R}$ & 4646430 & 2 & 6 & 15 & 0 \\
\hline $2 \mathrm{R}$ & 4649172 & 2 & 12 & 11 & 0 \\
\hline $2 \mathrm{R}$ & 4652266 & 2 & 7 & 5 & 0 \\
\hline $2 \mathrm{R}$ & 4652371 & 2 & 10 & 6 & 0 \\
\hline $2 R$ & 4655402 & 2 & 16 & 4 & 0 \\
\hline $2 \mathrm{R}$ & 4659690 & 2 & 13 & 14 & 0 \\
\hline $2 \mathrm{R}$ & 4661109 & 2 & 12 & 21 & 0 \\
\hline $2 \mathrm{R}$ & 4661363 & 2 & 17 & 33 & 0 \\
\hline $2 R$ & 4663676 & 2 & 30 & 7 & 0 \\
\hline
\end{tabular}




\begin{tabular}{|c|c|c|c|c|c|}
\hline $2 \mathrm{R}$ & 4664609 & 2 & 10 & 6 & 0 \\
\hline $2 R$ & 4672005 & 2 & 7 & 7 & 0 \\
\hline $2 R$ & 4673634 & 2 & 9 & 24 & 0 \\
\hline $2 \mathrm{R}$ & 4675760 & 2 & 11 & 15 & 0 \\
\hline $2 \mathrm{R}$ & 4679536 & 2 & 30 & 10 & 0 \\
\hline $2 \mathrm{R}$ & 4679720 & 2 & 9 & 7 & 0 \\
\hline $2 \mathrm{R}$ & 4680279 & 2 & 19 & 17 & 0 \\
\hline $2 R$ & 4681554 & 2 & 13 & 8 & 0 \\
\hline $2 \mathrm{R}$ & 4681981 & 2 & 15 & 10 & 0 \\
\hline $2 \mathrm{R}$ & 4682067 & 2 & 8 & 19 & 0 \\
\hline $2 \mathrm{R}$ & 4682158 & 2 & 19 & 16 & 0 \\
\hline $2 \mathrm{R}$ & 4682773 & 2 & 22 & 8 & 0 \\
\hline $2 \mathrm{R}$ & 4683142 & 2 & 6 & 16 & 0 \\
\hline $2 \mathrm{R}$ & 4684134 & 2 & 4 & 20 & 0 \\
\hline $2 \mathrm{R}$ & 4684993 & 2 & 24 & 6 & 0 \\
\hline $2 \mathrm{R}$ & 4685885 & 2 & 10 & 22 & 0 \\
\hline $2 R$ & 4686547 & 2 & 27 & 8 & 0 \\
\hline $2 \mathrm{R}$ & 4686760 & 2 & 18 & 8 & 0 \\
\hline $2 \mathrm{R}$ & 4687065 & 2 & 7 & 29 & 0 \\
\hline $2 R$ & 4687891 & 2 & 28 & 12 & 0 \\
\hline $2 \mathrm{R}$ & 4688825 & 2 & 19 & 33 & 0 \\
\hline $2 \mathrm{R}$ & 4688910 & 2 & 14 & 16 & 0 \\
\hline $2 \mathrm{R}$ & 4689320 & 2 & 18 & 5 & 0 \\
\hline $2 \mathrm{R}$ & 4691041 & 2 & 7 & 5 & 0 \\
\hline $2 \mathrm{R}$ & 4691257 & 2 & 6 & 14 & 0 \\
\hline $2 R$ & 4691285 & 2 & 7 & 6 & 0 \\
\hline $2 \mathrm{R}$ & 4691733 & 2 & 18 & 7 & 0 \\
\hline $2 \mathrm{R}$ & 4692383 & 2 & 11 & 23 & 0 \\
\hline $2 \mathrm{R}$ & 4693513 & 2 & 16 & 10 & 0 \\
\hline $2 \mathrm{R}$ & 4693622 & 2 & 5 & 7 & 0 \\
\hline $2 \mathrm{R}$ & 4694343 & 2 & 4 & 19 & 0 \\
\hline $2 R$ & 4694498 & 2 & 13 & 4 & 0 \\
\hline $2 R$ & 4695076 & 2 & 5 & 5 & 0 \\
\hline $2 \mathrm{R}$ & 4695137 & 2 & 12 & 6 & 0 \\
\hline $2 \mathrm{R}$ & 4695783 & 2 & 14 & 5 & 0 \\
\hline $2 \mathrm{R}$ & 4695853 & 2 & 13 & 5 & 0 \\
\hline $2 \mathrm{R}$ & 4695970 & 2 & 18 & 12 & 0 \\
\hline $2 \mathrm{R}$ & 4696570 & 2 & 21 & 11 & 0 \\
\hline $2 \mathrm{R}$ & 4698344 & 2 & 16 & 6 & 0 \\
\hline $2 \mathrm{R}$ & 4698706 & 2 & 22 & 20 & 0 \\
\hline $2 \mathrm{R}$ & 4700759 & 2 & 8 & 8 & 0 \\
\hline $2 \mathrm{R}$ & 4701420 & 2 & 15 & 8 & 0 \\
\hline $2 \mathrm{R}$ & 4701501 & 2 & 11 & 4 & 0 \\
\hline $2 R$ & 4702167 & 2 & 16 & 7 & 0 \\
\hline $2 \mathrm{R}$ & 4702185 & 2 & 11 & 13 & 0 \\
\hline $2 \mathrm{R}$ & 4702264 & 2 & 14 & 7 & 0 \\
\hline $2 R$ & 4702931 & 2 & 17 & 16 & 0 \\
\hline $2 \mathrm{R}$ & 4703523 & 2 & 4 & 17 & 0 \\
\hline $2 \mathrm{R}$ & 4704635 & 2 & 15 & 4 & 0 \\
\hline $2 R$ & 4704709 & 2 & 21 & 5 & 0 \\
\hline $2 \mathrm{R}$ & 4705135 & 2 & 16 & 6 & 0 \\
\hline $2 \mathrm{R}$ & 4705185 & 2 & 23 & 13 & 0 \\
\hline $2 R$ & 4707598 & 2 & 4 & 11 & 0 \\
\hline
\end{tabular}


$2 \mathrm{R}$

$2 R$

$2 R$

$2 R$

$2 R$

$2 R$

$2 \mathrm{R}$

$2 \mathrm{R}$

$2 \mathrm{R}$

$2 \mathrm{R}$

$2 \mathrm{R}$

$2 \mathrm{R}$

$2 \mathrm{R}$

$2 \mathrm{R}$

$2 \mathrm{R}$

$2 \mathrm{R}$

$2 \mathrm{R}$

$2 \mathrm{R}$

$2 \mathrm{R}$

$2 \mathrm{R}$

$2 \mathrm{R}$

$2 \mathrm{R}$

$2 \mathrm{R}$

$2 \mathrm{R}$

$2 \mathrm{R}$

2R

$2 \mathrm{R}$

$2 \mathrm{R}$

$2 \mathrm{R}$

$2 \mathrm{R}$

$2 \mathrm{R}$

$2 \mathrm{R}$

$2 \mathrm{R}$

$2 \mathrm{R}$

$2 \mathrm{R}$

$2 \mathrm{R}$

$2 \mathrm{R}$

$2 \mathrm{R}$

$2 \mathrm{R}$

$2 \mathrm{R}$

$2 \mathrm{R}$

$2 \mathrm{R}$

$2 \mathrm{R}$

$2 \mathrm{R}$

$2 \mathrm{R}$

$2 \mathrm{R}$

$2 \mathrm{R}$

$2 \mathrm{R}$

$2 \mathrm{R}$

$2 \mathrm{R}$

$2 \mathrm{R}$

$2 \mathrm{R}$

$2 \mathrm{R}$
4709566

4709760

4719279

4724439

4724574

4726147

4728597

4730573

4731077

4739280

4740262

4740683

4753256

4758668

4766145

4779025

4794423

4796295

4799432

4801550

4809725

4814797

4820309

4821067

4823787

4826061

4826290

4827093

4828580

4829149

4831141

4831273

4832198

4835014

4837720

4837993

4839146

4839406

4840963

4851707

4852559

4855675

4856828

4864063

4868835

4872015

4874699

4876987

4877490

4878209

4881718

4883905

4884769
2

2

2

2

2

2

2

2

2

2

2

2

2

2

2

2

2

2

2

2

2

2

2

2

2

2

2

2

2

2

2

2

2

2

2

2

2

2

2

2

2

2

2

2

2

2

2

2

2

2

2

2

2
19

18

20

5

18

14

7

9

4

7

26

12

9

28

6

14

22

15

20

14

6

6

7

28

12

10

16

10

6

18

17

12

8

12

34

13

6

20

12

10

7

19

17

4

6

16

6

8

5

21

6

10

14 $\begin{array}{ll}8 & 0 \\ 6 & 0\end{array}$

$9 \quad 0$

230

190

$18 \quad 0$

$15 \quad 0$

$4 \quad 0$

$11 \quad 0$

$5 \quad 0$

$16 \quad 0$

40

$4 \quad 0$

$17 \quad 0$

110

150

50

$6 \quad 0$

$7 \quad 0$

$24 \quad 0$

$\begin{array}{ll}9 & 0 \\ 2 & 0\end{array}$

$9 \quad 0$

$6 \quad 0$

26

26

6

16

12

15

7

4

10

7

9

6

15

6

6

16

16

9

19

8

5

14

27

8

17

10

4

0

0

0

0

0

0

0

0

0

0

0

0

0

0

0

0

0

0

0

0

0

0
0 
$2 R$

$2 R$

$2 \mathrm{R}$

$2 R$

$2 \mathrm{R}$

$2 R$

$2 \mathrm{R}$

$2 \mathrm{R}$

$2 \mathrm{R}$

$2 \mathrm{R}$

2R

$2 \mathrm{R}$

$2 \mathrm{R}$

$2 \mathrm{R}$

$2 \mathrm{R}$

$2 \mathrm{R}$

$2 \mathrm{R}$

$2 \mathrm{R}$

$2 \mathrm{R}$

$2 \mathrm{R}$

$2 \mathrm{R}$

$2 \mathrm{R}$

$2 \mathrm{R}$

$2 \mathrm{R}$

$2 \mathrm{R}$

$2 \mathrm{R}$

$2 \mathrm{R}$

$2 \mathrm{R}$

$2 \mathrm{R}$

$2 \mathrm{R}$

$2 \mathrm{R}$

$2 \mathrm{R}$

$2 \mathrm{R}$

$2 \mathrm{R}$

$2 \mathrm{R}$

$2 \mathrm{R}$

$2 \mathrm{R}$

$2 \mathrm{R}$

$2 \mathrm{R}$

$2 \mathrm{R}$

$2 \mathrm{R}$

$2 \mathrm{R}$

$2 \mathrm{R}$

$2 \mathrm{R}$

$2 \mathrm{R}$

$2 \mathrm{R}$

$2 \mathrm{R}$

$2 \mathrm{R}$

$2 \mathrm{R}$

$2 \mathrm{R}$

$2 \mathrm{R}$

$2 \mathrm{R}$

$2 \mathrm{R}$
4886001

4886886

4887321

4891833

4893649

4893736

4901452

4903685

4907246

4907567

4908337

4911303

4912301

4912531

4915257

4917380

4922139

4922988

4926591

4926653

4926750

4928195

4928336

4929760

4931400

4933910

4936572

4937612

4938888

4940486

4942391

4942420

4942733

4943713

4943844

4944686

4944816

4945452

4948040

4949324

4950597

4952590

4955426

4964342

4964909

4965351

4966352

4976191

4978623

4978640

4981322

4987386

4990312
2

2

2

2

2

2

2

2

2

2

2

2

2

2

2

2

2

2

2

2

2

2

2

2

2

2

2

2

2

2

2

2

2

2

2

2

2

2

2

2

2

2

2

2

2

2

2

2

2

2

2

2

2
9
22

16

16

16

7

17

17

15

19

20

4

13

8

9

8

11

21

14

25

8

16

10

15

12

11

5

21

10

19

21

20

5

16

14

24

16

16

6

32

4

7

5

15

5

7

13

4

21

16

16

4

28
8

10

11

10

13

9

9

5

8

9

6

16

6

16

14

8

19

16

17

12

5

9

5

4

11

60

7

17

6

7

15

10

11

5

10

22

10

4

7

9

22

7

10

7

29

19

8

21

6

7

23

23

18

0

0

0

0

$$
\begin{aligned}
& 0 \\
& 0
\end{aligned}
$$$$
0
$$$$
0
$$$$
0
$$$$
0
$$$$
0
$$$$
0
$$$$
0
$$$$
0
$$$$
0
$$$$
0
$$$$
0
$$$$
0
$$$$
0
$$ 


\begin{tabular}{|c|c|c|c|c|c|}
\hline $2 \mathrm{R}$ & 4990425 & 2 & 17 & 10 & 0 \\
\hline $2 \mathrm{R}$ & 4996709 & 2 & 20 & 4 & 0 \\
\hline $2 \mathrm{R}$ & 4998116 & 2 & 5 & 20 & 0 \\
\hline $2 R$ & 5001176 & 2 & 5 & 5 & 0 \\
\hline $2 \mathrm{R}$ & 5001406 & 2 & 5 & 6 & 0 \\
\hline $2 \mathrm{R}$ & 5002838 & 2 & 17 & 40 & 0 \\
\hline $2 \mathrm{R}$ & 5005210 & 2 & 13 & 4 & 0 \\
\hline $2 \mathrm{R}$ & 5005386 & 2 & 11 & 18 & 0 \\
\hline $2 \mathrm{R}$ & 5009262 & 2 & 14 & 12 & 0 \\
\hline $2 \mathrm{R}$ & 5010734 & 2 & 17 & 10 & 0 \\
\hline $2 R$ & 5010868 & 2 & 6 & 27 & 0 \\
\hline $2 R$ & 5015154 & 2 & 7 & 24 & 0 \\
\hline $2 R$ & 5022116 & 2 & 9 & 5 & 0 \\
\hline $2 \mathrm{R}$ & 5026158 & 2 & 19 & 12 & 0 \\
\hline $2 R$ & 5028193 & 2 & 12 & 25 & 0 \\
\hline $2 \mathrm{R}$ & 5028929 & 2 & 17 & 12 & 0 \\
\hline $2 \mathrm{R}$ & 5030388 & 2 & 11 & 7 & 0 \\
\hline $2 \mathrm{R}$ & 5031437 & 2 & 10 & 5 & 0 \\
\hline $2 R$ & 5033518 & 2 & 8 & 6 & 0 \\
\hline $2 \mathrm{R}$ & 5033590 & 2 & 10 & 5 & 0 \\
\hline $2 \mathrm{R}$ & 5046236 & 2 & 14 & 9 & 0 \\
\hline $2 R$ & 5050119 & 2 & 18 & 11 & 0 \\
\hline $2 \mathrm{R}$ & 5051742 & 2 & 17 & 9 & 0 \\
\hline $2 R$ & 5052534 & 2 & 9 & 20 & 0 \\
\hline $2 \mathrm{R}$ & 5053536 & 2 & 5 & 21 & 0 \\
\hline $2 \mathrm{R}$ & 5054362 & 2 & 6 & 9 & 0 \\
\hline $2 \mathrm{R}$ & 5070728 & 2 & 22 & 8 & 0 \\
\hline $2 R$ & 5073857 & 2 & 10 & 4 & 0 \\
\hline $2 \mathrm{R}$ & 5113193 & 2 & 9 & 35 & 0 \\
\hline $2 \mathrm{R}$ & 5114267 & 2 & 11 & 4 & 0 \\
\hline $2 R$ & 5114714 & 2 & 7 & 12 & 0 \\
\hline $2 \mathrm{R}$ & 5115020 & 2 & 14 & 4 & 0 \\
\hline $2 \mathrm{R}$ & 5116246 & 2 & 4 & 14 & 0 \\
\hline $2 \mathrm{R}$ & 5118618 & 2 & 26 & 13 & 0 \\
\hline $2 \mathrm{R}$ & 5120475 & 2 & 14 & 11 & 0 \\
\hline $2 R$ & 5123641 & 2 & 8 & 13 & 0 \\
\hline $2 R$ & 5123739 & 2 & 5 & 9 & 0 \\
\hline $2 \mathrm{R}$ & 5124002 & 2 & 16 & 5 & 0 \\
\hline $2 \mathrm{R}$ & 5127108 & 2 & 13 & 4 & 0 \\
\hline $2 \mathrm{R}$ & 5127659 & 2 & 11 & 8 & 0 \\
\hline $2 \mathrm{R}$ & 5128268 & 2 & 7 & 14 & 0 \\
\hline $2 \mathrm{R}$ & 5129245 & 2 & 19 & 9 & 0 \\
\hline $2 R$ & 5129382 & 2 & 20 & 7 & 0 \\
\hline $2 \mathrm{R}$ & 5131086 & 2 & 11 & 5 & 0 \\
\hline $2 \mathrm{R}$ & 5133306 & 2 & 14 & 12 & 0 \\
\hline $2 \mathrm{R}$ & 5134686 & 2 & 9 & 7 & 0 \\
\hline $2 \mathrm{R}$ & 5137019 & 2 & 5 & 12 & 0 \\
\hline $2 \mathrm{R}$ & 5137395 & 2 & 7 & 13 & 0 \\
\hline $2 R$ & 5137592 & 2 & 5 & 30 & 0 \\
\hline $2 \mathrm{R}$ & 5138887 & 2 & 5 & 14 & 0 \\
\hline $2 \mathrm{R}$ & 5138937 & 2 & 7 & 16 & 0 \\
\hline $2 \mathrm{R}$ & 5138967 & 2 & 6 & 8 & 0 \\
\hline $2 R$ & 5139013 & 2 & 19 & 10 & 0 \\
\hline
\end{tabular}




\begin{tabular}{|c|c|c|c|c|c|}
\hline $2 \mathrm{R}$ & 5139145 & 2 & 10 & 19 & 0 \\
\hline $2 \mathrm{R}$ & 5139179 & 2 & 10 & 27 & 0 \\
\hline $2 \mathrm{R}$ & 5139221 & 2 & 6 & 15 & 0 \\
\hline $2 R$ & 5140577 & 2 & 21 & 14 & 0 \\
\hline $2 \mathrm{R}$ & 5141234 & 2 & 26 & 31 & 0 \\
\hline $2 \mathrm{R}$ & 5141338 & 2 & 17 & 7 & 0 \\
\hline $2 \mathrm{R}$ & 5141614 & 2 & 7 & 15 & 0 \\
\hline $2 \mathrm{R}$ & 5142918 & 2 & 10 & 10 & 0 \\
\hline $2 \mathrm{R}$ & 5143146 & 2 & 13 & 14 & 0 \\
\hline $2 \mathrm{R}$ & 5143402 & 2 & 4 & 18 & 0 \\
\hline $2 \mathrm{R}$ & 5143472 & 2 & 4 & 14 & 0 \\
\hline $2 R$ & 5143796 & 2 & 9 & 4 & 0 \\
\hline $2 R$ & 5144773 & 2 & 11 & 7 & 0 \\
\hline $2 \mathrm{R}$ & 5145506 & 2 & 6 & 5 & 0 \\
\hline $2 R$ & 5145553 & 2 & 15 & 14 & 0 \\
\hline $2 \mathrm{R}$ & 5145595 & 2 & 37 & 17 & 0 \\
\hline $2 \mathrm{R}$ & 5145734 & 2 & 19 & 9 & 0 \\
\hline $2 \mathrm{R}$ & 5145775 & 2 & 17 & 13 & 0 \\
\hline $2 R$ & 5146768 & 2 & 32 & 9 & 0 \\
\hline $2 \mathrm{R}$ & 5146978 & 2 & 12 & 8 & 0 \\
\hline $2 \mathrm{R}$ & 5148101 & 2 & 16 & 8 & 0 \\
\hline $2 R$ & 5148182 & 2 & 16 & 7 & 0 \\
\hline $2 \mathrm{R}$ & 5148425 & 2 & 21 & 14 & 0 \\
\hline $2 R$ & 5148541 & 2 & 24 & 5 & 0 \\
\hline $2 \mathrm{R}$ & 5148728 & 2 & 14 & 12 & 0 \\
\hline $2 \mathrm{R}$ & 5149987 & 2 & 31 & 19 & 0 \\
\hline $2 \mathrm{R}$ & 5150228 & 2 & 12 & 15 & 0 \\
\hline $2 R$ & 5151432 & 2 & 10 & 6 & 0 \\
\hline $2 \mathrm{R}$ & 5151438 & 2 & 11 & 9 & 0 \\
\hline $2 \mathrm{R}$ & 5152092 & 2 & 4 & 20 & 0 \\
\hline $2 R$ & 5153685 & 2 & 4 & 20 & 0 \\
\hline $2 \mathrm{R}$ & 5156807 & 2 & 5 & 6 & 0 \\
\hline $2 \mathrm{R}$ & 5160577 & 2 & 7 & 25 & 0 \\
\hline $2 \mathrm{R}$ & 5162573 & 2 & 9 & 21 & 0 \\
\hline $2 \mathrm{R}$ & 5162656 & 2 & 5 & 19 & 0 \\
\hline $2 R$ & 5162728 & 2 & 7 & 9 & 0 \\
\hline $2 R$ & 5163361 & 2 & 8 & 18 & 0 \\
\hline $2 \mathrm{R}$ & 5163621 & 2 & 7 & 14 & 0 \\
\hline $2 \mathrm{R}$ & 5163777 & 2 & 7 & 15 & 0 \\
\hline $2 \mathrm{R}$ & 5164522 & 2 & 6 & 8 & 0 \\
\hline $2 \mathrm{R}$ & 5164839 & 2 & 20 & 5 & 0 \\
\hline $2 \mathrm{R}$ & 5164919 & 2 & 17 & 18 & 0 \\
\hline $2 R$ & 5165020 & 2 & 19 & 20 & 0 \\
\hline $2 \mathrm{R}$ & 5165093 & 2 & 20 & 17 & 0 \\
\hline $2 \mathrm{R}$ & 5165208 & 2 & 33 & 12 & 0 \\
\hline $2 \mathrm{R}$ & 5165634 & 2 & 6 & 24 & 0 \\
\hline $2 \mathrm{R}$ & 5168349 & 2 & 14 & 9 & 0 \\
\hline $2 \mathrm{R}$ & 5180257 & 2 & 4 & 9 & 0 \\
\hline $2 R$ & 5181681 & 2 & 15 & 6 & 0 \\
\hline $2 \mathrm{R}$ & 5181730 & 2 & 4 & 18 & 0 \\
\hline $2 \mathrm{R}$ & 5184428 & 2 & 18 & 14 & 0 \\
\hline $2 \mathrm{R}$ & 5185649 & 2 & 8 & 11 & 0 \\
\hline $2 \mathrm{R}$ & 5186712 & 2 & 26 & 5 & 0 \\
\hline
\end{tabular}




\begin{tabular}{|c|c|c|c|c|c|}
\hline $2 \mathrm{R}$ & 5188816 & 2 & 15 & 4 & 0 \\
\hline $2 R$ & 5188909 & 2 & 9 & 4 & 0 \\
\hline $2 \mathrm{R}$ & 5189210 & 2 & 15 & 9 & 0 \\
\hline $2 \mathrm{R}$ & 5190616 & 2 & 12 & 20 & 0 \\
\hline $2 \mathrm{R}$ & 5191382 & 2 & 23 & 9 & 0 \\
\hline $2 \mathrm{R}$ & 5191429 & 2 & 12 & 5 & 0 \\
\hline $2 \mathrm{R}$ & 5192535 & 2 & 8 & 30 & 0 \\
\hline $2 R$ & 5192953 & 2 & 24 & 18 & 0 \\
\hline $2 \mathrm{R}$ & 5195197 & 2 & 7 & 10 & 0 \\
\hline $2 \mathrm{R}$ & 5195251 & 2 & 14 & 10 & 0 \\
\hline $2 \mathrm{R}$ & 5196359 & 2 & 4 & 19 & 0 \\
\hline $2 \mathrm{R}$ & 5196501 & 2 & 7 & 8 & 0 \\
\hline $2 \mathrm{R}$ & 5197420 & 2 & 20 & 14 & 0 \\
\hline $2 \mathrm{R}$ & 5199316 & 2 & 4 & 9 & 0 \\
\hline $2 \mathrm{R}$ & 5201139 & 2 & 5 & 21 & 0 \\
\hline $2 \mathrm{R}$ & 5202830 & 2 & 8 & 18 & 0 \\
\hline $2 R$ & 5204212 & 2 & 11 & 16 & 0 \\
\hline $2 \mathrm{R}$ & 5205292 & 2 & 9 & 18 & 0 \\
\hline $2 \mathrm{R}$ & 5205475 & 2 & 6 & 7 & 0 \\
\hline $2 R$ & 5206138 & 2 & 9 & 12 & 0 \\
\hline $2 \mathrm{R}$ & 5207041 & 2 & 11 & 6 & 0 \\
\hline $2 \mathrm{R}$ & 5207191 & 2 & 13 & 10 & 0 \\
\hline $2 \mathrm{R}$ & 5208094 & 2 & 10 & 5 & 0 \\
\hline $2 \mathrm{R}$ & 5208096 & 2 & 10 & 6 & 0 \\
\hline $2 \mathrm{R}$ & 5208569 & 2 & 4 & 11 & 0 \\
\hline $2 \mathrm{R}$ & 5210965 & 2 & 14 & 7 & 0 \\
\hline $2 \mathrm{R}$ & 5210986 & 2 & 7 & 9 & 0 \\
\hline $2 \mathrm{R}$ & 5211612 & 2 & 12 & 9 & 0 \\
\hline $2 \mathrm{R}$ & 5214835 & 2 & 5 & 19 & 0 \\
\hline $2 \mathrm{R}$ & 5216784 & 2 & 8 & 14 & 0 \\
\hline $2 \mathrm{R}$ & 5217532 & 2 & 18 & 10 & 0 \\
\hline $2 \mathrm{R}$ & 5217623 & 2 & 18 & 9 & 0 \\
\hline $2 R$ & 5220015 & 2 & 4 & 14 & 0 \\
\hline $2 \mathrm{R}$ & 5220267 & 2 & 17 & 10 & 0 \\
\hline $2 \mathrm{R}$ & 5220702 & 2 & 16 & 15 & 0 \\
\hline $2 \mathrm{R}$ & 5221869 & 2 & 8 & 9 & 0 \\
\hline $2 \mathrm{R}$ & 5226277 & 2 & 9 & 4 & 0 \\
\hline $2 \mathrm{R}$ & 5226617 & 2 & 14 & 5 & 0 \\
\hline $2 \mathrm{R}$ & 5226933 & 2 & 8 & 5 & 0 \\
\hline $2 \mathrm{R}$ & 5227342 & 2 & 11 & 9 & 0 \\
\hline $2 \mathrm{R}$ & 5228435 & 2 & 20 & 16 & 0 \\
\hline $2 \mathrm{R}$ & 5228495 & 2 & 21 & 4 & 0 \\
\hline $2 \mathrm{R}$ & 5228590 & 2 & 18 & 21 & 0 \\
\hline $2 R$ & 5231430 & 2 & 14 & 21 & 0 \\
\hline $2 \mathrm{R}$ & 5232122 & 2 & 14 & 4 & 0 \\
\hline $2 \mathrm{R}$ & 5232576 & 2 & 26 & 9 & 0 \\
\hline $2 R$ & 5233297 & 2 & 20 & 4 & 0 \\
\hline $2 \mathrm{R}$ & 5233621 & 2 & 9 & 18 & 0 \\
\hline $2 \mathrm{R}$ & 5234075 & 2 & 18 & 16 & 0 \\
\hline $2 R$ & 5234520 & 2 & 10 & 31 & 0 \\
\hline $2 \mathrm{R}$ & 5234795 & 2 & 17 & 8 & 0 \\
\hline $2 \mathrm{R}$ & 5235538 & 2 & 7 & 16 & 0 \\
\hline $2 R$ & 5238453 & 2 & 10 & 7 & 0 \\
\hline
\end{tabular}




\begin{tabular}{|c|c|}
\hline $2 \mathrm{R}$ & 5238875 \\
\hline $2 \mathrm{R}$ & 5249347 \\
\hline $2 \mathrm{R}$ & 5250140 \\
\hline $2 \mathrm{R}$ & 5250444 \\
\hline $2 \mathrm{R}$ & 5250698 \\
\hline $2 \mathrm{R}$ & 5250948 \\
\hline $2 \mathrm{R}$ & 5251301 \\
\hline $2 \mathrm{R}$ & 5251977 \\
\hline $2 \mathrm{R}$ & 5255093 \\
\hline $2 \mathrm{R}$ & 5255110 \\
\hline $2 \mathrm{R}$ & 5256600 \\
\hline $2 \mathrm{R}$ & 5261900 \\
\hline $2 \mathrm{R}$ & 5263332 \\
\hline $2 \mathrm{R}$ & 5263473 \\
\hline $2 \mathrm{R}$ & 5269108 \\
\hline $2 \mathrm{R}$ & 5269601 \\
\hline $2 \mathrm{R}$ & 5269897 \\
\hline $2 \mathrm{R}$ & 5279323 \\
\hline $2 \mathrm{R}$ & 5279421 \\
\hline $2 \mathrm{R}$ & 5281621 \\
\hline $2 \mathrm{R}$ & 5283327 \\
\hline $2 \mathrm{R}$ & 5283768 \\
\hline $2 \mathrm{R}$ & 5283990 \\
\hline $2 \mathrm{R}$ & 5284647 \\
\hline $2 \mathrm{R}$ & 5284851 \\
\hline $2 \mathrm{R}$ & 5285898 \\
\hline $2 \mathrm{R}$ & 5286023 \\
\hline $2 \mathrm{R}$ & 5289394 \\
\hline $2 \mathrm{R}$ & 5289856 \\
\hline $2 \mathrm{R}$ & 5290126 \\
\hline $2 \mathrm{R}$ & 5293103 \\
\hline $2 \mathrm{R}$ & 5293456 \\
\hline $2 \mathrm{R}$ & 5293749 \\
\hline $2 \mathrm{R}$ & 5295110 \\
\hline $2 \mathrm{R}$ & 5296246 \\
\hline $2 \mathrm{R}$ & 5296632 \\
\hline $2 \mathrm{R}$ & 5297539 \\
\hline $2 \mathrm{R}$ & 5300112 \\
\hline $2 \mathrm{R}$ & 5300241 \\
\hline $2 \mathrm{R}$ & 5301420 \\
\hline $2 \mathrm{R}$ & 5302638 \\
\hline $2 \mathrm{R}$ & 5302709 \\
\hline $2 \mathrm{R}$ & 5303635 \\
\hline $2 \mathrm{R}$ & 5304129 \\
\hline $2 \mathrm{R}$ & 5304331 \\
\hline $2 \mathrm{R}$ & 5304539 \\
\hline $2 \mathrm{R}$ & 5304732 \\
\hline $2 \mathrm{R}$ & 5305954 \\
\hline $2 \mathrm{R}$ & 5306423 \\
\hline $2 \mathrm{R}$ & 5307444 \\
\hline $2 \mathrm{R}$ & 5310214 \\
\hline $2 \mathrm{R}$ & 5310983 \\
\hline 21 & 5313287 \\
\hline
\end{tabular}

\section{2}

2

2

2

2

2

2

2

2

2

2

2

2

2

2

2

2

2

2

2

2

2

2

2

2

2

2

2

2

2

2

2

2

2

2

2

2

2

2

2

2

2

2

2

2

2

2

2

2

2

2
9
14
6
13
6
8
11
5
7

11

12

8

11

8

10

4

4

17

11

26

14

9

6

25

10

12

4

7

9

18

5

4

22

10

10

19

15

7

9

27

28

28

18

5

4

24

6

12

5

8

7

25
9

31

14

21

10

16

23

29

11

13

6

31

12

14

9

19

21

17

8

10

17

6

10

8

20

4

13

14

4

4

9

21

8

10

5

4

4

23

10

6

14

5

4

20

15

18

15

39

13

5

6

12

11
0

0

0

0

0

0

0

0

0

0

0

0

0

0

0

0

0

0

0

0

0

0

0

0

0

0

0

0

0

0

0

0

0

0

0

0

0

0

0

0

0

0

0

0

0

0

0

0

0

0 
$2 \mathrm{R}$

$2 R$

$2 R$

$2 R$

$2 R$

$2 R$

$2 \mathrm{R}$

$2 \mathrm{R}$

$2 \mathrm{R}$

$2 \mathrm{R}$

$2 \mathrm{R}$

$2 \mathrm{R}$

$2 \mathrm{R}$

$2 \mathrm{R}$

$2 \mathrm{R}$

$2 \mathrm{R}$

$2 \mathrm{R}$

$2 \mathrm{R}$

$2 \mathrm{R}$

$2 \mathrm{R}$

$2 \mathrm{R}$

$2 \mathrm{R}$

$2 \mathrm{R}$

$2 \mathrm{R}$

$2 \mathrm{R}$

2R

$2 \mathrm{R}$

$2 \mathrm{R}$

$2 R$

$2 \mathrm{R}$

$2 \mathrm{R}$

$2 \mathrm{R}$

$2 \mathrm{R}$

$2 \mathrm{R}$

$2 \mathrm{R}$

$2 \mathrm{R}$

$2 \mathrm{R}$

$2 \mathrm{R}$

$2 \mathrm{R}$

$2 \mathrm{R}$

$2 \mathrm{R}$

$2 \mathrm{R}$

$2 \mathrm{R}$

$2 \mathrm{R}$

$2 \mathrm{R}$

$2 \mathrm{R}$

$2 \mathrm{R}$

$2 \mathrm{R}$

$2 \mathrm{R}$

$2 \mathrm{R}$

$2 \mathrm{R}$

$2 \mathrm{R}$

$2 \mathrm{R}$
5313925

5314063

5321762

5326019

5327128

5332789

5332906

5334246

5334281

5336853

5338611

5338730

5340023

5341512

5342249

5342322

5343999

5344045

5344316

5344401

5345020

5346498

5347396

5349423

5351601

5354366

5354892

5355492

5361718

5364333

5364734

5365591

5382073

5382247

5383153

5384034

5394057

5395594

7059535

7060850

7087689

7104715

7107191

7114493

7115670

7117748

7120049

7125347

7126328

7126386

7126889

7126912

7127614
2

2

2

2

2

2

2

2

2

2

2

2

2

2

2

2

2

2

2

2

2

2

2

2

2

2

2

2

2

2

2

2

2

2

2

2

2

2

2

2

2

2

2

2

2

2

2

2

2

2

2

2

2

$\begin{array}{rr}7 & 16 \\ 9 & 21 \\ 7 & 22 \\ 7 & 14 \\ 12 & 10 \\ 10 & 21 \\ 11 & \\ 7 & 12 \\ 5 & 20 \\ 4 & 21 \\ 20 & \end{array}$

10

15

19

18

23

12

19

15

26

4

6

12

7

22

4

5

20

29

5

18

13

20

13

12

20

23

16

7

13

14

11

13

28

19

17

5

14

11

14

$16 \quad 0$

210

22

14

10

21

6

12

20

21
6

11

12

11

7

16

6

13

10

8

13

25

11

10

19

12

8

6

4

17

17

4

11

10

7

5

4

6

6

4

6

8

5

9

8

8

5

20

11

4

5

7

11

0
0
0

0

0

0

0

0

0

0

0

0

0

0

0

0

0

0

0

0

0

0

0

0

0

0

0

0

0

0

0

0

0

0

0

0

0

0 


$\begin{array}{lr}2 R & 7130991 \\ 2 R & 7131627 \\ 2 R & 7133769 \\ 2 R & 7135452 \\ 2 R & 7136293 \\ 2 R & 7140393 \\ 2 R & 7140780 \\ 2 R & 7140872 \\ 2 R & 7164834 \\ 2 R & 7180142 \\ 2 R & 7217607 \\ 2 R & 7337511 \\ 2 R & 7386442 \\ 2 R & 7470544 \\ 2 R & 7482127 \\ 2 R & 7514871 \\ 2 R & 7692059 \\ 2 R & 8797425 \\ 2 R & 10050925 \\ 2 R & 10819403 \\ 2 R & 11564762 \\ 2 R & 12404110 \\ 2 R & 12538064 \\ 2 R & 12547208 \\ 2 R & 12557923 \\ 2 R & 12605941 \\ 2 R & 12645466 \\ 2 R & 12700340 \\ 2 R & 12785102 \\ 2 R & 12835342 \\ 2 R & 13052695 \\ 2 R & 13147264 \\ 2 R & 13148363 \\ 2 R & 13304982 \\ 2 R & 13357074 \\ 2 R & 13532631 \\ 2 R & 13648614 \\ 2 R & 13741244 \\ 2 R & 13741253 \\ 2 R & 13811347 \\ 2 R & 14223065 \\ 2 R & 15530974 \\ 2 R & 15546399 \\ 2 R & 16380626 \\ 2 R & 17162490 \\ 2 R & 17616067 \\ 2 R & 20712666 \\ 2 R & 20733085 \\ 2 R & 211057975 \\ 2 R & \end{array}$

2

2

2

2

2

2

2

2

2

2

2

2

2

2

2

2

2

2

2

2

2

2

2

2

2

2

2

2

2

2

2

2

2

2

2

2

2

2

2

2

2

2

2

2

2

2

2

2

2

2

2

2

2

2

20
12
11
8
6
12
4
13

12

19

6

5

6

6

5

5

10

6

5
5

5

8
11

14

7

11

6

11

6

4

4

6

15

7

6

6

12

9

7

4

5

6

7

5

9

5

9

14

4

4

10

5

7

4

5

6

5

$18 \quad 0$

120

40

$6 \quad 0$

$8 \quad 0$

140

50

$5 \quad 0$

$6 \quad 0$

$6 \quad 0$

$5 \quad 0$

$5 \quad 0$

$\begin{array}{ll}5 & 0 \\ 6 & 0\end{array}$

$15 \quad 0$

$16 \quad 0$

$4 \quad 0$

70

$\begin{array}{ll}5 & 0 \\ 5 & 0\end{array}$

$8-0$

$4 \quad 0$

7

10

9

5

5

7

6

5

6

5

6

13

5

5

5

6

4

5

7

4

10

11

5

9

5
0

0

0

0

0

0

0

0

0

0

0

0

0

0

0

0

0

0

0

0

0

0

0 
$3 \mathrm{~L}$

$3 L$

$3 \mathrm{~L}$

$3 L$

$3 L$

$3 L$

3L

3L

3L

3L

$3 \mathrm{~L}$

$3 \mathrm{~L}$

$3 \mathrm{~L}$

3L

3L

$3 \mathrm{~L}$

$3 \mathrm{~L}$

$3 \mathrm{~L}$

$3 \mathrm{~L}$

$3 \mathrm{~L}$

3L

$3 \mathrm{~L}$

$3 \mathrm{~L}$

$3 \mathrm{~L}$

$3 \mathrm{~L}$

$3 \mathrm{~L}$

$3 \mathrm{~L}$

$3 \mathrm{~L}$

$3 \mathrm{~L}$

$3 \mathrm{~L}$

$3 \mathrm{~L}$

$3 \mathrm{~L}$

$3 \mathrm{~L}$

$3 \mathrm{~L}$

3L

$3 \mathrm{~L}$

$3 \mathrm{~L}$

$3 \mathrm{~L}$

$3 \mathrm{~L}$

$3 \mathrm{~L}$

3L

$3 \mathrm{~L}$

$3 \mathrm{~L}$

3L

3L

3L

$3 \mathrm{~L}$

3L

3L

3L

3L

3L

3L
108478

131690

438017

448575

558331

558644

576704

657947

659520

712066

713342

715761

716881

726572

739431

747303

750449

769090

771543

772783

774684

776866

785681

824769

832489

832809

849718

876013

876300

877143

884197

901662

904355

912395

919237

919251

941253

951541

970880

979868

989965

1009880

1013462

1018697

1021528

1057464

1059626

1093446

1104315

1175733

1186435

1188020

1194193
2

2

2

2

2

2

2

2

2

2

2

2

2

2

2

2

2

2

2

2

2

2

2

2

2

2

2

2

2

2

2

2

2

2

2

2

2

2

2

2

2

2

2

2

2

2

2

2

2

2

2

2

2

5
4
6

$5 \quad 0$

$9 \quad 0$

$5 \quad 0$

11

6

9

5

9

12

10

4

10

6

$7 \quad 0$

$6 \quad 0$

$9 \quad 0$

$6 \quad 0$

110

110

$\begin{array}{ll}5 & 0 \\ 6 & 0\end{array}$

$9 \quad 0$

190

$16 \quad 0$

$36 \quad 0$

$13 \quad 0$

150

$10 \quad 0$

$\begin{array}{ll}9 & 0 \\ 7 & 0\end{array}$

140

$4 \quad 0$

$7 \quad 0$

$7 \quad 0$

$14 \quad 0$

$10 \quad 0$

$20 \quad 0$

$4 \quad 0$

$6 \quad 0$

$6 \quad 0$

$16 \quad 0$

$7 \quad 0$

120

$8 \quad 0$

110

110

7
8

13

11

14

5

14

0

0

0

0

12

7 
$3 L$

$3 L$

3L

$3 \mathrm{~L}$

$3 L$

$3 L$

3L

3L

3L

3L

3L

$3 \mathrm{~L}$

$3 \mathrm{~L}$

3L

$3 \mathrm{~L}$

$3 \mathrm{~L}$

$3 \mathrm{~L}$

$3 \mathrm{~L}$

$3 \mathrm{~L}$

3L

3L

$3 \mathrm{~L}$

$3 \mathrm{~L}$

$3 \mathrm{~L}$

$3 \mathrm{~L}$

$3 \mathrm{~L}$

$3 \mathrm{~L}$

$3 \mathrm{~L}$

$3 \mathrm{~L}$

$3 \mathrm{~L}$

$3 \mathrm{~L}$

$3 \mathrm{~L}$

$3 \mathrm{~L}$

$3 \mathrm{~L}$

3L

$3 \mathrm{~L}$

$3 \mathrm{~L}$

$3 \mathrm{~L}$

$3 \mathrm{~L}$

$3 \mathrm{~L}$

3L

$3 \mathrm{~L}$

$3 \mathrm{~L}$

3L

3L

3L

3L

3L

3L

3L

3L

3L

3L
1194275

1203538

1218696

1229832

1265616

1269689

1281477

1284321

1286937

1293506

1302519

1308320

1314196

1317899

1357349

1378354

1400319

1405798

1417124

1448691

1453215

1455030

1483038

1503317

1503677

1522125

1523973

1528870

1528874

1533743

1535806

1541761

1543351

1553466

1567305

1574178

1577602

1590235

1594812

1598874

1602557

1605791

1610427

1617049

1617064

1621133

1622890

1627079

1627965

1629240

1631045

1633923

1636207
2

2

2

2

2

2

2

2

2

2

2

2

2

2

2

2

2

2

2

2

2

2

2

2

2

2

2

2

2

2

2

2

2

2

2

2

2

2

2

2

2

2

2

2

2

2

2

2

2

2

2

2

2

$\begin{array}{rr}5 & 1 \\ 7 & \\ 7 & \\ 19 & 1 \\ 5 & 1 \\ 18 & 10 \\ 5 & 13\end{array}$

11

8

8

6

5

6

11

5

7

4

5
14

14

4

13

15

5

5

8

16

4

11

12

6

20

5

9

14

9

5

13

5

7

7

7

12

12

9

14

11

10

4

5

19

5

12
4
13

90

8

12

10

10

13
4

6

12

5

6

5

8

9

13

16

8

17

5

10

17

15

16

4

10

10

10

5

14

11

15

6

9

6

5

5

7

14

11

23

22

6

13

5

6

9

5

8

22

22

26

$$
0
$$$$
0
$$$$
0
$$$$
0
$$$$
0
$$$$
0
$$$$
0
$$$$
0
$$$$
0
$$$$
0
$$$$
0
$$$$
0
$$$$
0
$$$$
0
$$$$
0
$$$$
0
$$$$
0
$$$$
0
$$$$
0
$$$$
0
$$$$
0
$$$$
0
$$$$
0
$$$$
0
$$$$
0
$$$$
0
$$

0 
$3 L$

$3 L$

$3 \mathrm{~L}$

$3 L$

$3 L$

$3 L$

$3 \mathrm{~L}$

3L

3L

$3 \mathrm{~L}$

$3 \mathrm{~L}$

$3 \mathrm{~L}$

3L

3L

$3 \mathrm{~L}$

$3 \mathrm{~L}$

$3 \mathrm{~L}$

$3 \mathrm{~L}$

3L

3L

$3 \mathrm{~L}$

$3 \mathrm{~L}$

$3 \mathrm{~L}$

$3 \mathrm{~L}$

$3 \mathrm{~L}$

$3 \mathrm{~L}$

$3 \mathrm{~L}$

$3 \mathrm{~L}$

$3 \mathrm{~L}$

$3 \mathrm{~L}$

$3 \mathrm{~L}$

$3 \mathrm{~L}$

$3 \mathrm{~L}$

$3 \mathrm{~L}$

3L

$3 \mathrm{~L}$

$3 \mathrm{~L}$

$3 \mathrm{~L}$

$3 \mathrm{~L}$

$3 \mathrm{~L}$

3L

$3 \mathrm{~L}$

3L

3L

3L

$3 \mathrm{~L}$

3L

3L

3L

3L

3L

3L

3L
1640672

1643994

1647005

1647061

1649526

1654507

1657154

1657960

1661508

1661646

1662211

1664682

1666246

1669040

1670987

1673015

1673511

1674558

1675841

1676043

1678760

1679000

1679634

1681778

1687254

1688736

1688967

1695202

1698403

1699798

1700652

1701455

1701602

1701847

1702445

1705996

1707038

1708188

1708740

1710160

1713151

1716216

1717029

1717142

1717786

1718037

1719256

1720193

1720315

1720491

1722021

1722141

1722510
2

2

2

2

2

2

2

2

2

2

2

2

2

2

2

2

2

2

2

2

2

2

2

2

2

2

2

2

2

2

2

2

2

2

2

2

2

2

2

2

2

2

2

2

2

2

2

2

2

2

2

2

2

5
14
6
8
5

$6 \quad 0$

12

19

7

7

14

23

7

8

10

6

10

25

13

18

14

11

12

13

20

7

16

24

4

6

6

29

7

7

10

6

5

7

7

6

6

4

20

21

16

10

7

25

19

18

23

7

17

4

10

16

5

13

0

0

0

0

0

0

0

0

0

0

0

0

0

0

0

0

0

0

0

0

0

0

0

0

0

0

0

0

0

0

0

0

0

0

0

0

0

0

0

0

0

0

0
0

4
5

14 
$3 L$

$3 L$

$3 L$

$3 L$

$3 L$

$3 L$

$3 \mathrm{~L}$

$3 \mathrm{~L}$

3L

$3 \mathrm{~L}$

$3 \mathrm{~L}$

$3 \mathrm{~L}$

$3 \mathrm{~L}$

3L

3L

$3 \mathrm{~L}$

$3 \mathrm{~L}$

$3 \mathrm{~L}$

$3 L$

$3 \mathrm{~L}$

$3 \mathrm{~L}$

$3 \mathrm{~L}$

$3 \mathrm{~L}$

$3 \mathrm{~L}$

$3 \mathrm{~L}$

$3 \mathrm{~L}$

$3 \mathrm{~L}$

$3 \mathrm{~L}$

$3 \mathrm{~L}$

$3 \mathrm{~L}$

$3 \mathrm{~L}$

$3 \mathrm{~L}$

$3 \mathrm{~L}$

3L

3L

$3 \mathrm{~L}$

$3 \mathrm{~L}$

$3 \mathrm{~L}$

$3 \mathrm{~L}$

3L

$3 \mathrm{~L}$

3L

3L

3L

3L

3L

3L

3L

3L

3L

3L

3L
1723030

1723568

1724921

1729491

1729572

1729661

1731079

1731084

1731975

1733028

1733100

1734626

1734895

1735476

1737029

1737275

1738587

1739142

1739366

1739396

1742646

1742755

1744103

1759152

1759415

1763390

1764092

1765762

1766688

1767450

1768704

1771930

1775666

1775879

1775896

1776343

1777100

1777833

1779556

1780038

1783959

1784475

1784552

1784816

1784856

1786410

1787052

1787595

1789360

1790077

1790210

1791412

1794848
2

2

2

2

2

2

2

2

2

2

2

2

2

2

2

2

2

2

2

2

2

2

2

2

2

2

2

2

2

2

2

2

2

2

2

2

2

2

2

2

2

2

2

2

2

2

2

2

2

2

2

2

2

$\begin{array}{rr}4 & 18 \\ 7 & 24 \\ 8 & 25 \\ 5 & 13 \\ 4 & 19 \\ 4 & 14 \\ 10 & \\ 10 & \end{array}$

$4 \quad 17$

$\begin{array}{ll}7 & 6\end{array}$

6

18

5

5

8

7

5

10

14

6

6

4

10

14

23

7

20

10

9

9

12

8

7

17

17

5

13

9

6

6

12

17

6

5

11

7

17

4

12

12

10

15

21
$18 \quad 0$

240

$25 \quad 0$

130

190

$14 \quad 0$

$8 \quad 0$

$9 \quad 0$

$6 \quad 0$

240

120

$13 \quad 0$

$29 \quad 0$

$22 \quad 0$

$21 \quad 0$

$6 \quad 0$

$21 \quad 0$

$5 \quad 0$

$24 \quad 0$

120

120

$10 \quad 0$

$10-0$

$5 \quad 0$

$9 \quad 0$

$10 \quad 0$

$\begin{array}{rl}19 & 0 \\ 5 & 0\end{array}$

110

$20 \quad 0$

$10 \quad 0$

110

$6 \quad 0$

110

$4 \quad 0$

$8 \quad 0$

$14 \quad 0$

110

$40 \quad 0$

$6 \quad 0$

$13 \quad 0$

210

$21 \quad 0$

$32 \quad 0$

190

$6 \quad 0$

120

$7 \quad 0$

9

10 
$3 \mathrm{~L}$

$3 L$

$3 \mathrm{~L}$

$3 L$

$3 L$

$3 L$

3L

$3 \mathrm{~L}$

3L

3L

3L

$3 \mathrm{~L}$

$3 \mathrm{~L}$

3L

$3 \mathrm{~L}$

$3 \mathrm{~L}$

$3 \mathrm{~L}$

$3 \mathrm{~L}$

$3 \mathrm{~L}$

3L

3L

$3 \mathrm{~L}$

$3 \mathrm{~L}$

$3 \mathrm{~L}$

$3 \mathrm{~L}$

$3 \mathrm{~L}$

$3 \mathrm{~L}$

$3 \mathrm{~L}$

$3 \mathrm{~L}$

$3 \mathrm{~L}$

$3 \mathrm{~L}$

$3 \mathrm{~L}$

$3 \mathrm{~L}$

$3 \mathrm{~L}$

3L

$3 \mathrm{~L}$

$3 \mathrm{~L}$

$3 \mathrm{~L}$

$3 \mathrm{~L}$

$3 \mathrm{~L}$

3L

$3 \mathrm{~L}$

$3 \mathrm{~L}$

3L

3L

$3 \mathrm{~L}$

3L

3L

3L

3L

3L

3L

3L
1798384

1798511

1799271

1803869

1805422

1809333

1812100

1813377

1817848

1818098

1828632

1833752

1834323

1834690

1834851

1839604

1840428

1840439

1840816

1841461

1843625

1844038

1851797

1852753

1854038

1855185

1855470

1855548

1855874

1856810

1857016

1860894

1862064

1863498

1863777

1867074

1867601

1870616

1873782

1874310

1874328

1875230

1880384

1884972

1892105

1893597

1894998

1906516

1912194

1913582

1921175

1922176

1922334
2

2

2

2

2

2

2

2

2

2

2

2

2

2

2

2

2

2

2

2

2

2

2

2

2

2

2

2

2

2

2

2

2

2

2

2

2

2

2

2

2

2

2

2

2

2

2

2

2

2

2

2

2

$\begin{array}{rr}4 & 26 \\ 4 & \\ 15 & \\ 14 & \\ 10 & 18 \\ 6 & \\ 7 & \end{array}$

$26 \quad 0$

$15 \quad 0$

8

18

10

5
7

18

10

17

10

13

8

21

23

6

15

16

4

8

7

8

6

14

15

18

15

17

6

28

27

15

5

22

11

19

5

4

24

23

21

15

10

15

6

6

7

7

17

12

9

7

5
0

0

0

0

0

0

0

0

0

0

0

0

0

0

0

0

0

0

0

0

0

0

0

0

0

0

0

0

0

0

0

0

0

0

0

0

0

0

0

0

0

0

0

0

0

0 
$3 \mathrm{~L}$

1927379

$3 L$

$3 L$

$3 L$

$3 L$

$3 \mathrm{~L}$

$3 \mathrm{~L}$

3L

3L

$3 \mathrm{~L}$

3L

$3 \mathrm{~L}$

3L

$3 \mathrm{~L}$

$3 \mathrm{~L}$

$3 \mathrm{~L}$

$3 \mathrm{~L}$

$3 \mathrm{~L}$

$3 \mathrm{~L}$

3L

$3 \mathrm{~L}$

$3 \mathrm{~L}$

$3 \mathrm{~L}$

$3 \mathrm{~L}$

$3 \mathrm{~L}$

$3 \mathrm{~L}$

$3 \mathrm{~L}$

$3 \mathrm{~L}$

$3 \mathrm{~L}$

$3 \mathrm{~L}$

$3 \mathrm{~L}$

$3 \mathrm{~L}$

$3 \mathrm{~L}$

$3 \mathrm{~L}$

$3 \mathrm{~L}$

$3 \mathrm{~L}$

$3 \mathrm{~L}$

$3 \mathrm{~L}$

$3 \mathrm{~L}$

$3 \mathrm{~L}$

3L

$3 \mathrm{~L}$

$3 \mathrm{~L}$

3L

3L

$3 \mathrm{~L}$

$3 \mathrm{~L}$

3L

3L

3L

3L

3L

3L
1932199

1932212

1934429

1934562

1935039

1935903

1935909

1936174

1936376

1942844

1943883

1944019

1944635

1944925

1959012

1959096

1959317

1959379

1960649

1961754

1969287

1973191

1973811

1976088

1980375

1982308

1989211

1991394

2006300

2013370

2014683

2015888

2017309

2021527

2021543

2022424

2022519

2039711

2056108

2058503

2073490

2080795

2082431

2088408

2096181

2099493

2106318

2123859

2135028

2135037

2135154

2135529
2

2

2

2

2

2

2

2

2

2

2

2

2

2

2

2

2

2

2

2

2

2

2

2

2

2

2

2

2

2

2

2

2

2

2

2

2

2

2

2

2

2

2

2

2

2

2

2

2

2

2

2

2
12

8

7

8

43

5

6

6

17

12

10

5

19

21

23

9

11

10

4

7

6

20

12

18

11

8

11

6

11

5

6

9

7

10

8

11

21

26

5

7

12

10

11

13

19

5

6

7

14

6

6

10
4

12

10

13

24

9

8

5

15

8

16

15

9

17

19

30

6

16

13

9

5
10

10

4
17

17
14

11

14

4

17

5

5

6

8

13

9

12

7

12

5

8

22

4

9

7

8

12

8

8

10

16

26

0

0

0

0

0

0

0

0

0

0

0

0

0
0

0

0

0

0

0

0

0

0

0

0 
$3 L$

$3 L$

$3 \mathrm{~L}$

$3 \mathrm{~L}$

$3 L$

$3 L$

3L

3L

3L

$3 \mathrm{~L}$

$3 \mathrm{~L}$

$3 \mathrm{~L}$

3L

3L

3L

$3 \mathrm{~L}$

3L

$3 \mathrm{~L}$

$3 \mathrm{~L}$

$3 \mathrm{~L}$

3L

$3 \mathrm{~L}$

$3 \mathrm{~L}$

$3 \mathrm{~L}$

$3 \mathrm{~L}$

$3 \mathrm{~L}$

$3 \mathrm{~L}$

$3 \mathrm{~L}$

$3 \mathrm{~L}$

$3 \mathrm{~L}$

$3 \mathrm{~L}$

$3 \mathrm{~L}$

$3 \mathrm{~L}$

$3 \mathrm{~L}$

3L

$3 \mathrm{~L}$

$3 \mathrm{~L}$

$3 \mathrm{~L}$

$3 \mathrm{~L}$

$3 \mathrm{~L}$

3L

$3 \mathrm{~L}$

$3 \mathrm{~L}$

3L

3L

3L

$3 \mathrm{~L}$

3L

3L

3L

3L

3L

3L
2135532

2141551

2147142

2150102

2153903

2161344

2172619

2174292

2201825

2211459

2230053

2232586

2233165

2234266

2235774

2253640

2254539

2255756

2257694

2259387

2259626

2283378

2284289

2310079

2313912

2319492

2334949

2343170

2352243

2354455

2355156

2358537

2360223

2365889

2374849

2376745

2378253

2379733

2385287

2396898

2399553

2400873

2403097

2403232

2403272

2406484

2410408

2415699

2422018

2422142

2423534

2428793

2434114
2

2

2

2

2

2

2

2

2

2

2

2

2

2

2

2

2

2

2

2

2

2

2

2

2

2

2

2

2

2

2

2

2

2

2

2

2

2

2

2

2

2

2

2

2

2

2

2

2

2

2

2

2

$\begin{array}{ll}5 & \\ 5 & 14 \\ 5 & 15 \\ 6 & \\ 4 & 18 \\ 8 & \end{array}$

33

4

5
14

6

6

16

9

12

5

4

15

13

10

26

4

8

8

14

20

4

6

11

13

9

16

17

4

8

4

10

17

16

12

8

7

14

15

11

17

7

4

7

12

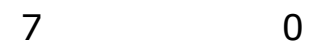

1

14
15

0

6

18
5

0
0

0

$9 \quad 0$

$10 \quad 0$

$7 \quad 0$

$6 \quad 0$

$6 \quad 0$

$7 \quad 0$

$7 \quad 0$

$8 \quad 0$

$7 \quad 0$

$22 \quad 0$

$10 \quad 0$

140

$6 \quad 0$

$4 \quad 0$

$7 \quad 0$

$17 \quad 0$

$10 \quad 0$

$19 \quad 0$

$\begin{array}{ll}7 & 0 \\ 9 & 0\end{array}$

140

$6 \quad 0$

$4 \quad 0$

$15 \quad 0$

10

6

11

9

12

13

5

5

5

7

8

5

4

13

7

10

10

14

7

9

11

5

10

0

0

0

0

0

0

0

0

0

0

0

0

0

0

0

0

0

6
6
8

8 
$3 L$

$3 L$

$3 L$

$3 \mathrm{~L}$

$3 L$

$3 L$

3L

3L

3L

3L

3L

$3 \mathrm{~L}$

$3 \mathrm{~L}$

$3 \mathrm{~L}$

$3 L$

3L

3L

$3 \mathrm{~L}$

$3 \mathrm{~L}$

$3 \mathrm{~L}$

3L

$3 \mathrm{~L}$

$3 \mathrm{~L}$

$3 \mathrm{~L}$

$3 \mathrm{~L}$

$3 \mathrm{~L}$

$3 \mathrm{~L}$

$3 \mathrm{~L}$

$3 \mathrm{~L}$

$3 \mathrm{~L}$

$3 \mathrm{~L}$

$3 \mathrm{~L}$

$3 \mathrm{~L}$

$3 \mathrm{~L}$

$3 \mathrm{~L}$

$3 \mathrm{~L}$

$3 \mathrm{~L}$

$3 \mathrm{~L}$

$3 \mathrm{~L}$

$3 \mathrm{~L}$

3L

$3 \mathrm{~L}$

3L

3L

3L

3L

3L

3L

3L

3L

3L

3L

3L
2435884

2435893

2437498

2438358

2438407

2439782

2458180

2463342

2466023

2473416

2486476

2488995

2492218

2492924

2493772

2496877

2496892

2503079

2503397

2505582

2505794

2520937

2522103

2540154

2549085

2562064

2562631

2567680

2569315

2570856

2574896

2575862

2578961

2583132

2588383

2595609

2598056

2599510

2600314

2606197

2606217

2608648

2619165

2619193

2631871

2633467

2633534

2634614

2635715

2638446

2639019

2641656

2642560
2

2

2

2

2

2

2

2

2

2

2

2

2

2

2

2

2

2

2

2

2

2

2

2

2

2

2

2

2

2

2

2

2

2

2

2

2

2

2

2

2

2

2

2

2

2

2

2

2

2

2

2

2
7

6

5

13

5

16

6

6

14

10

4

8

5

17

9

12

6

8

11

9

13

9

11

16

6

10

8

4

15

18

11

12

5

7

9

10

16

5

6

10

9

7

11

11

13

13

20

13

6

19

8

18

9
8

10

8

4

6

18

9

27

4

6

11

8

8

4
10

24

29

6

4

8

8

4

7

9

6

5

5

13

9

9

6

7

7

7

13

5

12

5

6

10

9

5

5

19

9

4

7

8

8

10

21

9

11

0

0

0

0

0

0

0

0

0

0

0

0

0

0

0

0

0

0

0

0

0 
$3 L$

$3 L$

$3 L$

$3 L$

$3 L$

$3 L$

3L

3L

3L

$3 \mathrm{~L}$

$3 \mathrm{~L}$

$3 \mathrm{~L}$

$3 \mathrm{~L}$

$3 \mathrm{~L}$

3L

$3 \mathrm{~L}$

3L

$3 \mathrm{~L}$

$3 \mathrm{~L}$

$3 \mathrm{~L}$

$3 \mathrm{~L}$

$3 \mathrm{~L}$

$3 \mathrm{~L}$

$3 \mathrm{~L}$

$3 \mathrm{~L}$

$3 \mathrm{~L}$

$3 \mathrm{~L}$

$3 \mathrm{~L}$

$3 \mathrm{~L}$

$3 \mathrm{~L}$

$3 \mathrm{~L}$

$3 \mathrm{~L}$

$3 \mathrm{~L}$

$3 \mathrm{~L}$

3L

$3 \mathrm{~L}$

$3 \mathrm{~L}$

$3 \mathrm{~L}$

$3 \mathrm{~L}$

$3 \mathrm{~L}$

3L

$3 \mathrm{~L}$

3L

3L

3L

3L

3L

3L

3L

3L

3L

3L

3L
2660018

2660024

2660374

2662128

2666509

2675028

2685570

2688900

2696887

2710368

2711256

2712852

2713728

2714413

2715678

2716888

2725909

2726125

2727363

2741659

2743112

2747059

2750474

2750949

2760010

2763798

2765982

2766702

2767561

2767579

2770538

2772806

2773225

2774230

2778349

2785665

2788366

2789467

2790396

2793598

2794571

2799950

2813691

2817856

2822188

2828870

2830463

2834048

2834990

2835400

2838309

2848828

2856098
2

2

2

2

2

2

2

2

2

2

2

2

2

2

2

2

2

2

2

2

2

2

2

2

2

2

2

2

2

2

2

2

2

2

2

2

2

2

2

2

2

2

2

2

2

2

2

2

2

2

2

2

2

$\begin{array}{rr}9 & 1 \\ 7 & 1 \\ 51 & \\ 21 & 1 \\ 5 & 1 \\ 15 & \\ 11 & \\ 7 & \\ 9 & \\ 7 & \end{array}$

12

12

16

5

8

17

19

12

15

10

4

16

8

6

11

5

14

8

10

14

15

7

21

9

5

14

19

10

7

120

140

4

12

11

7
7

5

$25 \quad 0$

$8 \quad 0$

11

11

4

18

10

8

15

8

5

12

21

4

8

10

7

6

4

5

5

8

6

13

6

6

14

12

6

17

15

10

4

13

6

7

8

4

12

5

4

11

18

7

6

7

13

0
0
0
0
0
0
0
0
0
0
0

0

0

0

0

0

0

0

0

0

0

0

0

0

0

0

0

0

0

0

0

0

0

0

0

0

0

0

0

0

0

0

0

0

0

0

11

8
25

31 
$3 L$

$3 L$

$3 \mathrm{~L}$

$3 L$

$3 L$

$3 L$

$3 \mathrm{~L}$

3L

3L

3L

3L

$3 \mathrm{~L}$

$3 \mathrm{~L}$

3L

$3 \mathrm{~L}$

3L

3L

$3 \mathrm{~L}$

$3 \mathrm{~L}$

$3 \mathrm{~L}$

$3 \mathrm{~L}$

$3 \mathrm{~L}$

$3 \mathrm{~L}$

$3 \mathrm{~L}$

$3 \mathrm{~L}$

$3 \mathrm{~L}$

$3 \mathrm{~L}$

$3 \mathrm{~L}$

$3 \mathrm{~L}$

$3 \mathrm{~L}$

$3 \mathrm{~L}$

$3 \mathrm{~L}$

$3 \mathrm{~L}$

$3 \mathrm{~L}$

3L

$3 \mathrm{~L}$

$3 L$

$3 \mathrm{~L}$

$3 \mathrm{~L}$

$3 \mathrm{~L}$

3L

$3 \mathrm{~L}$

3L

3L

$3 \mathrm{~L}$

$3 \mathrm{~L}$

3L

$3 \mathrm{~L}$

3L

3L

3L

3L

3L
2859558

2867275

2867646

2868878

2873963

2874039

2882277

2882766

2882777

2882865

2886847

2889892

2902251

2902455

2905075

2908433

2915999

2917109

2920621

2921482

2924011

2938034

2942869

2945474

2955764

2957766

2958437

2959712

2963891

2964169

2979085

2981656

2984445

2984458

2987109

2988412

2989277

3004782

3006863

3006865

3007190

3008718

3008755

3008931

3009288

3022314

3032551

3048476

3048727

3051466

3055224

3057405

3057978
2

2

2

2

2

2

2

2

2

2

2

2

2

2

2

2

2

2

2

2

2

2

2

2

2

2

2

2

2

2

2

2

2

2

2

2

2

2

2

2

2

2

2

2

2

2

2

2

2

2

2

2

2
6

5

9

18

14

14

8

12

12

14

10

10

10

4

11

10

11

6

7

5

9

18

14

6

8

7

7

8

17

8

13

10

10

5

9

7

9

9

9

5

7

8

10

5

4

16

10

11

18

10

14

14

14
18

7

15

6

6

6

13

14

11

7

11

7

12

21

5

4

5
17

16

14

7

21

11

21

18

9

6

10

31

8

19

10

5

8

4

16

4

5

5

5

26

25

17

12

18

10

14

13

10

6

5

13

6

0

0

0

0

0

0

0

0

0

0

0

0

0

0

0

0

0

0

0

0

0 
$3 L$

$3 L$

$3 L$

$3 L$

$3 L$

$3 \mathrm{~L}$

3L

3L

3L

$3 \mathrm{~L}$

$3 \mathrm{~L}$

$3 \mathrm{~L}$

$3 \mathrm{~L}$

3L

$3 \mathrm{~L}$

$3 \mathrm{~L}$

3L

$3 \mathrm{~L}$

$3 \mathrm{~L}$

$3 \mathrm{~L}$

3L

$3 \mathrm{~L}$

$3 \mathrm{~L}$

$3 \mathrm{~L}$

$3 \mathrm{~L}$

$3 \mathrm{~L}$

$3 \mathrm{~L}$

$3 \mathrm{~L}$

$3 \mathrm{~L}$

$3 \mathrm{~L}$

$3 \mathrm{~L}$

$3 \mathrm{~L}$

$3 \mathrm{~L}$

$3 \mathrm{~L}$

$3 \mathrm{~L}$

$3 \mathrm{~L}$

$3 \mathrm{~L}$

$3 \mathrm{~L}$

$3 \mathrm{~L}$

$3 \mathrm{~L}$

3L

$3 \mathrm{~L}$

3L

3L

3L

3L

$3 \mathrm{~L}$

3L

3L

3L

3L

3L

3L
3079550

3081310

3082631

3083118

3101133

3102510

3102741

3107349

3107581

3110278

3115472

3124463

3125351

3126967

3132714

3133477

3135468

3136286

3139007

3140125

3144093

3145251

3145362

3148261

3154693

3165345

3171040

3190117

3190168

3192952

3197437

3200485

3200811

3205560

3205692

3207707

3211852

3237294

3248706

3271200

3273610

3276156

3279526

3287660

3287947

3288239

3294635

3294914

3296715

3307754

3311366

3313160

3316302
2

2

2

2

2

2

2

2

2

2

2

2

2

2

2

2

2

2

2

2

2

2

2

2

2

2

2

2

2

2

2

2

2

2

2

2

2

2

2

2

2

2

2

2

2

2

2

2

2

2

2

2

2

9
9
12
27
11
28
7
10

16

13

5

14

4

11

13

5

12

4

12

6

17

15

18

13

8

12

12

13

11

17

8

4

6

4

4

7

6

8

23

15

8

15

11

8

14

11

9

8

17

22

4

6

12
9

$4 \quad 0$

$4 \quad 0$

50

$5 \quad 0$

$8 \quad 0$

$8 \quad 0$

$9 \quad 0$

130

$15 \quad 0$

$10 \quad 0$

$\begin{array}{ll}14 & 0 \\ 10 & 0\end{array}$

100

$\begin{array}{ll}4 & 0 \\ 5 & 0\end{array}$

$7 \quad 0$

$6 \quad 0$

$16 \quad 0$

$10 \quad 0$

$6 \quad 0$

$8 \quad 0$

$\begin{array}{ll}7 & 0 \\ 4 & 0\end{array}$

$5 \quad 0$

$16 \quad 0$

$8 \quad 0$

$5 \quad 0$

7

11

16

18

26

19

13

14

27

18

9

4

14

5

6

5

21

26

16

5

12

10

9

13

17
0
0
0
0
0
0
0
0
0
0
0
0
0
0
0
0
0
0
0
0
0
0
0
0
0
0

0

0

0

0

0

0

0

0

0

0

0

0

0

0

0

0

0

0

0

0

0

0
0 
$3 \mathrm{~L}$

$3 L$

$3 L$

$3 \mathrm{~L}$

$3 L$

$3 L$

3L

3L

3L

$3 \mathrm{~L}$

$3 \mathrm{~L}$

$3 \mathrm{~L}$

3L

3L

$3 \mathrm{~L}$

$3 L$

3L

$3 \mathrm{~L}$

$3 \mathrm{~L}$

$3 \mathrm{~L}$

3L

$3 \mathrm{~L}$

$3 \mathrm{~L}$

$3 \mathrm{~L}$

$3 \mathrm{~L}$

$3 \mathrm{~L}$

$3 \mathrm{~L}$

$3 \mathrm{~L}$

$3 \mathrm{~L}$

$3 \mathrm{~L}$

$3 \mathrm{~L}$

$3 \mathrm{~L}$

$3 \mathrm{~L}$

$3 \mathrm{~L}$

3L

$3 \mathrm{~L}$

$3 \mathrm{~L}$

$3 \mathrm{~L}$

$3 L$

$3 \mathrm{~L}$

3L

$3 \mathrm{~L}$

$3 \mathrm{~L}$

3L

3L

$3 \mathrm{~L}$

3L

3L

3L

3L

3L

3L

3L
3319622

3323547

3327895

3332774

3335646

3336324

3347724

3349642

3365544

3376716

3411188

3413382

3423097

3454673

3469931

3492919

3495067

3495087

3497447

3501628

3501642

3508869

3513608

3516843

3518764

3519439

3525734

3532034

3532216

3532322

3534541

3538035

3540116

3543718

3547111

3550580

3552360

3556945

3557132

3558860

3571257

3571411

3575995

3579191

3585061

3586159

3586930

3589435

3596412

3599993

3609955

3611446

3613116
2

2

2

2

2

2

2

2

2

2

2

2

2

2

2

2

2

2

2

2

2

2

2

2

2

2

2

2

2

2

2

2

2

2

2

2

2

2

2

2

2

2

2

2

2

2

2

2

2

2

2

2

2
14

6

10

9

9

15

11

8

7

5

8

12

4

8
20

13

10

7

5

5

5

6

10

13

6

7

8

10

7

12

18

4

7

4

13

4

9

6

6

9

4

8

7

4

5

15

10

12

28

24

12

$6 \quad 0$

230

$15 \quad 0$

140

$5 \quad 0$

$7 \quad 0$

$4 \quad 0$

$19 \quad 0$

$22 \quad 0$

$13 \quad 0$

110

$4 \quad 0$

$21 \quad 0$

$6 \quad 0$

$9 \quad 0$

$\begin{array}{rr}4 & 0 \\ 10 & 0\end{array}$

$7 \quad 0$

$6 \quad 0$

$16 \quad 0$

120

$9 \quad 0$

$11 \quad 0$

$\begin{array}{ll}4 & 0 \\ 5 & 0\end{array}$

$22-0$

120

$12 \quad 0$

$20 \quad 0$

$10 \quad 0$

120

$14 \quad 0$

$10-0$

$8 \quad 0$

110

140

$9 \quad 0$

$7 \quad 0$

$5 \quad 0$

150

$5 \quad 0$

$7 \quad 0$

110

$5 \quad 0$

$6 \quad 0$

$4 \quad 0$

120

$13 \quad 0$

$10 \quad 0$

11

8

0 
$3 L$

$3 L$

$3 L$

$3 L$

$3 L$

$3 L$

$3 \mathrm{~L}$

3L

3L

$3 \mathrm{~L}$

$3 \mathrm{~L}$

$3 \mathrm{~L}$

3L

3L

$3 \mathrm{~L}$

$3 \mathrm{~L}$

3L

$3 \mathrm{~L}$

$3 \mathrm{~L}$

$3 \mathrm{~L}$

3L

$3 \mathrm{~L}$

$3 \mathrm{~L}$

$3 \mathrm{~L}$

$3 \mathrm{~L}$

$3 \mathrm{~L}$

$3 \mathrm{~L}$

$3 \mathrm{~L}$

$3 \mathrm{~L}$

$3 \mathrm{~L}$

$3 \mathrm{~L}$

$3 \mathrm{~L}$

$3 \mathrm{~L}$

$3 \mathrm{~L}$

$3 \mathrm{~L}$

$3 \mathrm{~L}$

$3 \mathrm{~L}$

$3 \mathrm{~L}$

$3 \mathrm{~L}$

$3 \mathrm{~L}$

3L

$3 \mathrm{~L}$

3L

3L

3L

$3 \mathrm{~L}$

3L

3L

$3 \mathrm{~L}$

3L

3L

3L

3L
3615735

3616835

3618319

3632915

3647347

3647714

3662332

3670926

3673226

3680385

3683539

3690317

3690461

3693444

3706892

3718360

3719836

3721062

3721392

3722802

3727842

3728208

3728357

3729004

3732943

3733315

3735339

3756604

3756971

3762352

3773343

3775155

3786453

3787623

3788397

3788482

3788757

3791339

3792347

3793179

3793680

3804118

3812499

3812901

3827109

3844186

3845451

3846418

3848700

3851170

3852931

3867274

3872384
2

2

2

2

2

2

2

2

2

2

2

2

2

2

2

2

2

2

2

2

2

2

2

2

2

2

2

2

2

2

2

2

2

2

2

2

2

2

2

2

2

2

2

2

2

2

2

2

2

2

2

2

2
17

7

7

14

7

4

13

13

5

4

6

4

5

4

11

15

11

10

4

4

8

13

21

8

11

9

13

11

4

7

12

7

8

8

17

5

4

6

4

8

11

6

9

6

7

22

6

6

10

5

10

12

10
$4 \quad 0$

$9 \quad 0$

13

16

16

16

5

5

5

17

18

15

7

9

7

5

4
18

$18 \quad 0$

$22 \quad 0$

$16 \quad 0$

50

$\begin{array}{ll}4 & 0 \\ 6 & 0\end{array}$

120

110

40

$5 \quad 0$

5

12

12

21

11

9

8

5

10

14

20

12

9

16

10

9

7

11

14

13

8

18

25

4

5

0
0
0
0
0
0
0
0
0
0
0
0

0

0

0

0

0

0

0

0

0

0

0

0

0

0

0

0

0

0

0

0 
$3 L$

$3 L$

$3 L$

$3 L$

$3 L$

$3 L$

3L

3L

3L

$3 \mathrm{~L}$

3L

$3 \mathrm{~L}$

$3 \mathrm{~L}$

3L

$3 \mathrm{~L}$

$3 \mathrm{~L}$

$3 \mathrm{~L}$

$3 \mathrm{~L}$

3L

$3 L$

3L

$3 \mathrm{~L}$

$3 \mathrm{~L}$

$3 \mathrm{~L}$

$3 \mathrm{~L}$

$3 \mathrm{~L}$

$3 \mathrm{~L}$

$3 \mathrm{~L}$

$3 \mathrm{~L}$

$3 \mathrm{~L}$

$3 \mathrm{~L}$

$3 \mathrm{~L}$

$3 \mathrm{~L}$

$3 \mathrm{~L}$

$3 \mathrm{~L}$

$3 \mathrm{~L}$

$3 L$

$3 \mathrm{~L}$

$3 \mathrm{~L}$

$3 \mathrm{~L}$

3L

$3 \mathrm{~L}$

3L

3L

3L

$3 \mathrm{~L}$

3L

$3 \mathrm{~L}$

3L

3L

3L

3L

3L
3872883

3886668

3893440

3898835

3903216

3905057

3909449

3924456

3930839

3938507

3944294

3946142

3947275

3953934

3975101

3976345

3981020

3987578

3997146

4007566

4008680

4008699

4020755

4030399

4032926

4035868

4035899

4043336

4045887

4050761

4055997

4060905

4061013

4064981

4065619

4067064

4079493

4080002

4093904

4113153

4114059

4116952

4117826

4130115

4131099

4143576

4173226

4173896

4185048

4185711

4215024

4224817

4235875
2

2

2

2

2

2

2

2

2

2

2

2

2

2

2

2

2

2

2

2

2

2

2

2

2

2

2

2

2

2

2

2

2

2

2

2

2

2

2

2

2

2

2

2

2

2

2

2

2

2

2

2

2

$\begin{aligned} 8 & \\ 7 & \\ 4 & \\ 8 & \\ 9 & \\ 10 & \\ 9 & \end{aligned}$

$8 \quad 0$

$9 \quad 0$

12

8

$7 \quad 0$

$7 \quad 0$

$14 \quad 0$

$6 \quad 0$

$10 \quad 0$

250

$9 \quad 0$

40

$19 \quad 0$

$7 \quad 0$

$\begin{array}{ll}5 & 0 \\ 5 & 0\end{array}$

$\begin{array}{ll}5 & 0 \\ 10 & 0\end{array}$

$6 \quad 0$

230

$14 \quad 0$

120

$8 \quad 0$

$17-0$

$11-0$

$7 \quad 0$

$16 \quad 0$

$7 \quad 0$

12

$4 \quad 0$

110

$16 \quad 0$

40

- 0

$-0$

$8 \quad 0$

$10 \quad 0$

$6 \quad 0$

$16 \quad 0$

50

90

40

$5 \quad 0$

90

$9 \quad 0$

11

16

12 
$3 L$

$3 L$

$3 \mathrm{~L}$

$3 L$

$3 L$

$3 L$

$3 \mathrm{~L}$

3L

3L

3L

3L

$3 \mathrm{~L}$

$3 \mathrm{~L}$

3L

3L

$3 \mathrm{~L}$

3L

$3 \mathrm{~L}$

$3 \mathrm{~L}$

$3 \mathrm{~L}$

3L

$3 \mathrm{~L}$

$3 \mathrm{~L}$

3L

$3 \mathrm{~L}$

$3 \mathrm{~L}$

$3 \mathrm{~L}$

$3 \mathrm{~L}$

$3 \mathrm{~L}$

$3 \mathrm{~L}$

$3 \mathrm{~L}$

$3 \mathrm{~L}$

$3 \mathrm{~L}$

$3 \mathrm{~L}$

3L

$3 \mathrm{~L}$

$3 \mathrm{~L}$

$3 \mathrm{~L}$

$3 \mathrm{~L}$

$3 \mathrm{~L}$

3L

$3 \mathrm{~L}$

$3 \mathrm{~L}$

3L

3L

3L

3L

3L

3L

$3 \mathrm{~L}$

3L

3L

3L
4268427

4268791

4283446

4289491

4289935

4290193

4290235

4290664

4314807

4315104

4315526

4325881

4343452

4348864

4349863

4352106

4355895

4359361

4359513

4365251

4366085

4368193

4368446

4377406

4383107

4389344

4391130

4392642

4400812

4404707

4432304

4462457

4469234

4471585

4487068

4500091

4506555

4512233

4512248

4520847

4528462

4529752

4534289

4564595

4566576

4566986

4588207

4595407

4599849

4601765

4609739

4610515

4617056
2

2

2

2

2

2

2

2

2

2

2

2

2

2

2

2

2

2

2

2

2

2

2

2

2

2

2

2

2

2

2

2

2

2

2

2

2

2

2

2

2

2

2

2

2

2

2

2

2

2

2

2

2
8

9

17

5

7

9

7

4

17

7

12

4

4

7

7

7

8

4

4

7

10

13

5

11

19

6

18

6

11

16

14

13

6

9

21

24

13

14

11

10

17

4

11

11

11

9

32

12

14

10

5

9

12
18

6

8

16

27

16

12

25

4

6

10

12

10

7

11

16

8

12

10

7

8

6

10

13

10

12

9

20

4

11

4

12

4

13

15

6

11

20

4

14

16

11

6

9

13

18

21

5

4

8

5

14

0

0

0

0

0

0

0

0

0

0

0

0

0

0

0

0

0

0

0

0

0 
$3 L$

$3 L$

$3 \mathrm{~L}$

$3 L$

$3 L$

$3 L$

$3 \mathrm{~L}$

$3 \mathrm{~L}$

3L

3L

3L

$3 \mathrm{~L}$

3L

3L

$3 \mathrm{~L}$

3L

3L

$3 \mathrm{~L}$

$3 \mathrm{~L}$

$3 \mathrm{~L}$

3L

$3 \mathrm{~L}$

$3 \mathrm{~L}$

$3 \mathrm{~L}$

$3 \mathrm{~L}$

$3 \mathrm{~L}$

$3 \mathrm{~L}$

$3 \mathrm{~L}$

$3 \mathrm{~L}$

$3 \mathrm{~L}$

$3 \mathrm{~L}$

$3 \mathrm{~L}$

$3 \mathrm{~L}$

$3 \mathrm{~L}$

3L

$3 \mathrm{~L}$

$3 \mathrm{~L}$

$3 \mathrm{~L}$

$3 \mathrm{~L}$

$3 \mathrm{~L}$

3L

$3 \mathrm{~L}$

3L

3L

3L

$3 \mathrm{~L}$

3L

3L

3L

3L

3L

3L

3L
4621192

4634838

4644820

4646257

4647639

4647708

4650621

4653783

4662124

4673456

4676265

4691566

4691654

4703681

4715547

4716077

4726485

4737028

4737461

4737511

4738364

4740090

4740098

4741182

4750309

4757116

4768992

4781498

4785291

4789733

4799819

4803380

4806154

4823682

4827576

4830994

4840737

4854515

4856179

4856466

4885413

4916798

4918793

4918902

4922501

4950603

4955925

4957939

4962462

4972263

4974589

5082819

5093761
2

2

2

2

2

2

2

2

2

2

2

2

2

2

2

2

2

2

2

2

2

2

2

2

2

2

2

2

2

2

2

2

2

2

2

2

2

2

2

2

2

2

2

2

2

2

2

2

2

2

2

2

2
11

5

13

12

17

5

18

28

4

4

9

5

5

5

5

7

11

5

18

5

13

13

4

5

10

8

5

8

8

5

9

4

10

14

15

13

8

4

15

11

12

7

24

13

11

4

5

6

12

6

4

10

19
9

17

23

11

17

9

12

15

5

24

15

23

6

8

11

4

5

4

11

11

4

5

25

13

9

11

13

11

8

5

6

6

22

13

7

4

12

7

11

7

15

16

8

6

4

8

13

6

6

6

0

0

0

0

0

0

0

0

0

0

0

0

0

0

0

0

0

0

0

0

0

0

0 
$3 \mathrm{~L}$

$3 L$

$3 L$

$3 L$

$3 L$

$3 L$

3L

3L

3L

3L

3L

$3 \mathrm{~L}$

$3 \mathrm{~L}$

3L

3L

$3 \mathrm{~L}$

$3 \mathrm{~L}$

$3 \mathrm{~L}$

$3 \mathrm{~L}$

$3 L$

3L

$3 \mathrm{~L}$

$3 \mathrm{~L}$

$3 \mathrm{~L}$

$3 \mathrm{~L}$

$3 \mathrm{~L}$

$3 \mathrm{~L}$

$3 \mathrm{~L}$

$3 \mathrm{~L}$

$3 \mathrm{~L}$

$3 \mathrm{~L}$

$3 \mathrm{~L}$

$3 \mathrm{~L}$

$3 \mathrm{~L}$

$3 \mathrm{~L}$

$3 \mathrm{~L}$

$3 \mathrm{~L}$

$3 \mathrm{~L}$

$3 \mathrm{~L}$

$3 \mathrm{~L}$

3L

$3 \mathrm{~L}$

3L

3L

3L

$3 \mathrm{~L}$

3L

$3 \mathrm{~L}$

3L

3L

3L

$3 \mathrm{~L}$

3L
5097377

5101323

5104460

5172852

5187354

5195303

5404385

5413933

5481015

5492425

5496689

5511664

5521447

5543268

5587787

5638149

5645791

5675659

5752965

5786057

5823334

5885896

5886659

5899376

5910335

5917392

5922554

5939474

6011258

6016707

6064411

6066508

6092565

6119920

6155454

6259059

6259735

6282767

6300596

6312756

6338603

6448623

6536398

6566453

6576136

6619629

6634214

6644335

6711568

6749186

6753325

6794403

6820031
2

2

2

2

2

2

2

2

2

2

2

2

2

2

2

2

2

2

2

2

2

2

2

2

2

2

2

2

2

2

2

2

2

2

2

2

2

2

2

2

2

2

2

2

2

2

2

2

2

2

2

2

2

$\begin{array}{rr}7 & \\ 17 & 1 \\ 8 & \\ 6 & \\ 7 & 2 \\ 8 & \\ 12 & \\ 6 & \\ 5 & \end{array}$

17

5

8

8

9

15

11

6

7

16

5

8

13

5

4

15

10

5

11

6

6

10

10

9

9

6

12

11

8 -

11

5

5

21

5

7

10

$4 \quad 0$

6

11

5

8

9

5

6

5

6

6

12

7

9

4

12

9

8

6

6

6

6

6

4

5

4

11

10

22

22

9

11

5

7

6

7

5

13

11

5

6

14

5

0
0
0
0
0
0
0
0
0
0

0

0

0

0

0

0

0

0

0

0

0

0

0

0

0

0

0

0

0

0

0

0

0

0

0

0

0

0

0

0

0

0

0

0

0

5

6 
$3 \mathrm{~L}$

$3 L$

$3 \mathrm{~L}$

$3 L$

$3 L$

$3 L$

3L

3L

3L

3L

3L

$3 \mathrm{~L}$

$3 \mathrm{~L}$

3L

3L

$3 \mathrm{~L}$

3L

$3 \mathrm{~L}$

$3 \mathrm{~L}$

$3 \mathrm{~L}$

3L

$3 \mathrm{~L}$

$3 \mathrm{~L}$

$3 \mathrm{~L}$

$3 \mathrm{~L}$

$3 \mathrm{~L}$

$3 \mathrm{~L}$

$3 \mathrm{~L}$

$3 \mathrm{~L}$

$3 \mathrm{~L}$

$3 \mathrm{~L}$

$3 \mathrm{~L}$

$3 \mathrm{~L}$

$3 \mathrm{~L}$

$3 \mathrm{~L}$

$3 \mathrm{~L}$

$3 \mathrm{~L}$

$3 \mathrm{~L}$

$3 \mathrm{~L}$

$3 \mathrm{~L}$

3L

$3 \mathrm{~L}$

3L

3L

3L

3L

3L

3L

3L

3L

3L

3L

3L
6835015

7140210

7501123

7591432

7837268

8003189

8396030

8639346

8803273

8830298

8912159

9000977

10624371

10794234

11627234

13017287

14951911

15821377

17478714

18360891

18924622

18928306

18955358

18958068

18959790

18959958

18960203

19011791

19020074

19032638

19037206

19038340

19062863

19065589

19066641

19084377

19097149

19119592

19123515

19132069

19157025

19174461

19178467

19182884

19185222

19208795

19235922

19249262

19258689

19282500

19285762

19326857

19337030
2

2

2

2

2

2

2

2

2

2

2

2

2

2

2

2

2

2

2

2

2

2

2

2

2

2

2

2

2

2

2

2

2

2

2

2

2

2

2

2

2

2

2

2

2

2

2

2

2

2

2

2

2

5
6
4
5
8
11

11

8

10

8

6

9
19

5

5

5

5

10

6

7

15

6

5

6

11

4

6

8

12

7

21

7

5

9

16

12

10

6

7

26

14

9

8

9

5

12

11

8

6

5

6

6

7

13

11

17

18

5

9
0

0

0

0

0

0

0

0

0

0

0

0

0

0

0

0

0

0

0

0

0

0

0

0

0

0

0

0

0

0

0

0

0

0

0

0

0

0

0

0

0

0

0

0

0

10

13

10 
$3 L$

$3 L$

$3 L$

$3 L$

$3 L$

$3 L$

3L

3L

3L

3L

3L

$3 \mathrm{~L}$

$3 \mathrm{~L}$

3L

3L

3L

3L

$3 \mathrm{~L}$

$3 \mathrm{~L}$

$3 \mathrm{~L}$

3L

$3 \mathrm{~L}$

$3 \mathrm{~L}$

3L

$3 \mathrm{~L}$

$3 \mathrm{~L}$

$3 \mathrm{~L}$

$3 \mathrm{~L}$

$3 \mathrm{~L}$

$3 \mathrm{~L}$

$3 \mathrm{~L}$

$3 \mathrm{~L}$

$3 \mathrm{~L}$

$3 \mathrm{~L}$

$3 \mathrm{~L}$

$3 \mathrm{~L}$

$3 \mathrm{~L}$

$3 \mathrm{~L}$

$3 \mathrm{~L}$

$3 \mathrm{~L}$

3L

$3 \mathrm{~L}$

3L

3L

3L

3L

3L

3L

3L

3L

3L

3L

3L
19429218

19447725

19457159

19492291

19658887

19850821

19916911

19952604

20046641

20049258

20049344

20057369

20061015

20077885

20088687

20095756

20142432

20163359

20204278

20212064

20231729

20255821

20286506

20306174

20339421

20406236

20407457

20408468

20449226

20467709

20478193

20661974

20669732

20710240

20718546

20858481

20900260

20900406

21045625

21054501

21063560

21185102

21200757

21204342

21222522

21229786

21249779

21282407

21365562

21409971

21456269

21569832

21583604
2

2

2

2

2

2

2

2

2

2

2

2

2

2

2

2

2

2

2

2

2

2

2

2

2

2

2

2

2

2

2

2

2

2

2

2

2

2

2

2

2

2

2

2

2

2

2

2

2

2

2

2

2

$\begin{array}{rr}9 & 12 \\ 10 & \\ 6 & \\ 9 & \\ 9 & \\ 6 & \\ 21 & \\ 8 & \end{array}$

120

$5 \quad 0$

7

4

6

12
8

$6 \quad 0$

120

15

18

4

6

5

20

8

10

9

6

5

6

8

6

5

8

4

9

12

10

6

31

4

4

10

8

11

5

12

6

5

7

9

29

9

14

5

11

4

10

4

5

5

4
7

9

9

6

11

26

$7 \quad 0$

$7 \quad 0$

40

$6 \quad 0$

$6 \quad 0$

$8 \quad 0$

$\begin{array}{ll}22 & 0 \\ 13 & 0\end{array}$

$21 \quad 0$

21

8

4

20

14

10

9

11

6

6

5

5

7

5

14

7

4

5

5

5

7

6

15

5

13

5

16

19

0

0

0

0

0

0

0

0

0

0

0

0

0

0

0

0

0

0

0

0

0

0

0

0

0
$6 \quad 0$ 
$3 \mathrm{~L}$

$3 L$

$3 L$

$3 \mathrm{~L}$

$3 L$

$3 L$

3L

3L

3L

$3 \mathrm{~L}$

$3 \mathrm{~L}$

$3 \mathrm{~L}$

$3 \mathrm{~L}$

3L

$3 \mathrm{~L}$

$3 \mathrm{~L}$

3L

$3 \mathrm{~L}$

$3 \mathrm{~L}$

$3 \mathrm{~L}$

3L

$3 \mathrm{~L}$

$3 \mathrm{~L}$

$3 \mathrm{~L}$

$3 \mathrm{~L}$

$3 \mathrm{~L}$

$3 \mathrm{~L}$

$3 \mathrm{~L}$

$3 \mathrm{~L}$

$3 \mathrm{~L}$

$3 \mathrm{~L}$

$3 \mathrm{~L}$

$3 \mathrm{~L}$

$3 \mathrm{~L}$

$3 \mathrm{~L}$

$3 \mathrm{~L}$

$3 \mathrm{~L}$

$3 \mathrm{~L}$

$3 \mathrm{~L}$

$3 \mathrm{~L}$

3L

$3 \mathrm{~L}$

$3 \mathrm{~L}$

3L

3L

3L

3L

3L

3L

3L

3L

3L

3L
21622273

21625080

21627016

21629670

21631208

21632185

21657439

21663937

21668796

21772176

21773671

21797113

21850174

21883641

21884717

21920735

21926271

21928511

21973356

21996031

21998210

22056493

22115189

22115196

22115469

22158157

22170833

22187901

22205376

22207224

22234470

22248593

22273050

22304042

22307227

22314782

22346505

22358743

22378307

22393962

22454899

22463789

22488317

22586300

22606238

22609193

22627639

22628885

22631538

22631610

22631673

22645479

22650605
2

2

2

2

2

2

2

2

2

2

2

2

2

2

2

2

2

2

2

2

2

2

2

2

2

2

2

2

2

2

2

2

2

2

2

2

2

2

2

2

2

2

2

2

2

2

2

2

2

2

2

2

2

15
15
6
5
6
23
16
5
8
11

10

8

4

7

5

24

16

10

8

9

8

10

8

5

8

4

17

13

7

18

14

13

10

11

9

14

8

12

9

20

8

12

4

12

23

7

7

8

6

14

15

8
$6 \quad 0$

$9 \quad 0$

7

$7 \quad 0$

$10 \quad 0$

140

$5 \quad 0$

$9 \quad 0$

$5 \quad 0$

$4 \quad 0$

50

$25 \quad 0$

$9 \quad 0$

$21 \quad 0$

$11 \quad 0$

$5 \quad 0$

$\begin{array}{ll}11 & 0 \\ 4 & 0\end{array}$

$6 \quad 0$

$10 \quad 0$

$5 \quad 0$

$11-0$

6

110

$9 \quad 0$

$\begin{array}{ll}9 & 0 \\ 7 & 0\end{array}$

$5 \quad 0$

7

12

4

4

4

4

4

11

10

9

5

6

5

16

9

7

4

6

15

7

11

6

7

10
0

0

0

0

0

0

0

0

0

0

0

0

0

0

0

0

0

0

0

0

0

0
0 
$3 L$

$3 L$

$3 L$

$3 L$

$3 L$

$3 L$

$3 \mathrm{~L}$

$3 \mathrm{~L}$

3L

3L

$3 \mathrm{~L}$

$3 \mathrm{~L}$

$3 \mathrm{~L}$

$3 \mathrm{~L}$

3L

$3 \mathrm{~L}$

$3 \mathrm{~L}$

$3 \mathrm{~L}$

$3 \mathrm{~L}$

3L

3L

3L

3L

3L

3L

$3 \mathrm{~L}$

$3 \mathrm{~L}$

$3 \mathrm{~L}$

$3 \mathrm{~L}$

$3 \mathrm{~L}$

$3 \mathrm{~L}$

$3 R$

$3 R$

$3 R$

$3 R$

$3 R$

$3 R$

$3 R$

$3 R$

$3 R$

$3 R$

$3 R$

$3 R$

$3 R$

$3 R$

$3 R$

$3 R$

$3 R$

$3 R$

$3 R$

$3 R$

$3 R$

$3 R$
22667716

22677848

22682330

22746063

22839245

22881580

22899207

22899380

22920116

22940041

22960178

22962733

23051871

23061964

23146999

23224329

23256285

23393609

23448250

23463788

23471445

23496681

23605025

23713341

23735341

23841621

23958072

24178032

24329353

24520727

24530497

30845

129054

146485

173772

201358

223996

348786

348851

354810

367961

368261

370997

372921

377395

384261

386873

396766

397139

398931

400475

401025

402782
2

2

2

2

2

2

2

2

2

2

2

2

2

2

2

2

2

2

2

2

2

2

2

2

2

2

2

2

2

2

2

2

2

2

2

2

2

2

2

2

2

2

2

2

2

2

2

2

2

2

2

2

2

$\begin{array}{rr}7 & 10 \\ 6 & 11 \\ 10 & 11 \\ 7 & \\ 9 & 4\end{array}$

10

110

110

$7 \quad 0$

$4 \quad 0$

$22 \quad 0$

$7 \quad 0$

$17 \quad 0$

$4 \quad 0$

$6 \quad 0$

$10 \quad 0$

$11 \quad 0$

$6 \quad 0$

$5 \quad 0$

$17 \quad 0$

140

$8 \quad 0$

120

120

$5 \quad 0$

$6 \quad 0$

$17 \quad 0$

120

$\begin{array}{ll}5 & 0 \\ 11 & 0\end{array}$

110

$5 \quad 0$

$8 \quad 0$

$4 \quad 0$

$7 \quad 0$

33

110

$23 \quad 0$

$13 \quad 0$

190

$10 \quad 0$

$10 \quad 0$

$6 \quad 0$

$26 \quad 0$

50

$8 \quad 0$

40

$5 \quad 0$

$5 \quad 0$

10

13

5

8

12

18
0

0

0

0 


\begin{tabular}{|c|c|c|c|c|c|}
\hline $3 R$ & 414074 & 2 & 12 & 9 & 0 \\
\hline $3 R$ & 416643 & 2 & 5 & 5 & 0 \\
\hline $3 R$ & 417713 & 2 & 18 & 7 & 0 \\
\hline $3 R$ & 417809 & 2 & 8 & 6 & 0 \\
\hline $3 R$ & 419144 & 2 & 14 & 5 & 0 \\
\hline $3 R$ & 420348 & 2 & 13 & 8 & 0 \\
\hline $3 R$ & 422867 & 2 & 13 & 9 & 0 \\
\hline $3 R$ & 432657 & 2 & 7 & 6 & 0 \\
\hline $3 R$ & 439292 & 2 & 8 & 9 & 0 \\
\hline $3 R$ & 441372 & 2 & 12 & 9 & 0 \\
\hline $3 R$ & 446388 & 2 & 10 & 5 & 0 \\
\hline $3 R$ & 446713 & 2 & 21 & 8 & 0 \\
\hline $3 R$ & 446750 & 2 & 9 & 4 & 0 \\
\hline $3 R$ & 456051 & 2 & 9 & 10 & 0 \\
\hline $3 R$ & 457008 & 2 & 9 & 5 & 0 \\
\hline $3 R$ & 458972 & 2 & 12 & 5 & 0 \\
\hline $3 R$ & 466382 & 2 & 14 & 6 & 0 \\
\hline $3 R$ & 468409 & 2 & 17 & 10 & 0 \\
\hline $3 R$ & 474358 & 2 & 8 & 5 & 0 \\
\hline $3 R$ & 475240 & 2 & 12 & 18 & 0 \\
\hline $3 R$ & 486393 & 2 & 18 & 12 & 0 \\
\hline $3 R$ & 490656 & 2 & 10 & 13 & 0 \\
\hline $3 R$ & 490795 & 2 & 14 & 4 & 0 \\
\hline $3 R$ & 498327 & 2 & 8 & 21 & 0 \\
\hline $3 R$ & 504941 & 2 & 7 & 12 & 0 \\
\hline $3 R$ & 506554 & 2 & 13 & 12 & 0 \\
\hline $3 R$ & 511618 & 2 & 4 & 28 & 0 \\
\hline $3 R$ & 529962 & 2 & 12 & 6 & 0 \\
\hline $3 R$ & 561354 & 2 & 8 & 5 & 0 \\
\hline $3 R$ & 570114 & 2 & 11 & 8 & 0 \\
\hline $3 R$ & 570979 & 2 & 11 & 6 & 0 \\
\hline $3 R$ & 590126 & 2 & 5 & 25 & 0 \\
\hline $3 R$ & 610453 & 2 & 11 & 5 & 0 \\
\hline $3 R$ & 625904 & 2 & 6 & 17 & 0 \\
\hline $3 R$ & 663695 & 2 & 18 & 4 & 0 \\
\hline $3 R$ & 675512 & 2 & 5 & 7 & 0 \\
\hline $3 R$ & 792829 & 2 & 4 & 13 & 0 \\
\hline $3 R$ & 802955 & 2 & 6 & 13 & 0 \\
\hline $3 R$ & 803647 & 2 & 8 & 12 & 0 \\
\hline $3 R$ & 821920 & 2 & 4 & 13 & 0 \\
\hline $3 R$ & 825322 & 2 & 9 & 7 & 0 \\
\hline $3 R$ & 838365 & 2 & 10 & 24 & 0 \\
\hline $3 R$ & 839321 & 2 & 9 & 5 & 0 \\
\hline $3 R$ & 852209 & 2 & 4 & 11 & 0 \\
\hline $3 R$ & 879490 & 2 & 14 & 9 & 0 \\
\hline $3 R$ & 880399 & 2 & 11 & 5 & 0 \\
\hline $3 R$ & 886023 & 2 & 9 & 5 & 0 \\
\hline $3 R$ & 886146 & 2 & 5 & 9 & 0 \\
\hline $3 R$ & 907147 & 2 & 5 & 5 & 0 \\
\hline $3 R$ & 932325 & 2 & 9 & 6 & 0 \\
\hline $3 R$ & 934374 & 2 & 11 & 21 & 0 \\
\hline $3 R$ & 951517 & 2 & 13 & 10 & 0 \\
\hline $3 R$ & 952339 & 2 & 5 & 8 & 0 \\
\hline
\end{tabular}




\begin{tabular}{|c|c|c|c|c|c|}
\hline $3 R$ & 960522 & 2 & 8 & 6 & 0 \\
\hline $3 R$ & 972526 & 2 & 11 & 11 & 0 \\
\hline $3 R$ & 976374 & 2 & 12 & 23 & 0 \\
\hline $3 R$ & 996022 & 2 & 6 & 7 & 0 \\
\hline $3 R$ & 999585 & 2 & 8 & 10 & 0 \\
\hline $3 R$ & 1011406 & 2 & 4 & 16 & 0 \\
\hline $3 R$ & 1026043 & 2 & 16 & 12 & 0 \\
\hline $3 R$ & 1036976 & 2 & 16 & 7 & 0 \\
\hline $3 R$ & 1046590 & 2 & 14 & 4 & 0 \\
\hline $3 R$ & 1057633 & 2 & 11 & 5 & 0 \\
\hline $3 R$ & 1070257 & 2 & 14 & 8 & 0 \\
\hline $3 R$ & 1074737 & 2 & 10 & 4 & 0 \\
\hline $3 R$ & 1083742 & 2 & 10 & 18 & 0 \\
\hline $3 R$ & 1109319 & 2 & 13 & 8 & 0 \\
\hline $3 R$ & 1116113 & 2 & 10 & 13 & 0 \\
\hline $3 R$ & 1116811 & 2 & 16 & 14 & 0 \\
\hline $3 R$ & 1125241 & 2 & 7 & 6 & 0 \\
\hline $3 R$ & 1130075 & 2 & 6 & 9 & 0 \\
\hline $3 R$ & 1132347 & 2 & 23 & 16 & 0 \\
\hline $3 R$ & 1132985 & 2 & 12 & 4 & 0 \\
\hline $3 R$ & 1141222 & 2 & 8 & 15 & 0 \\
\hline $3 R$ & 1151890 & 2 & 7 & 6 & 0 \\
\hline $3 R$ & 1189081 & 2 & 26 & 11 & 0 \\
\hline $3 R$ & 1199299 & 2 & 11 & 7 & 0 \\
\hline $3 R$ & 1210780 & 2 & 8 & 5 & 0 \\
\hline $3 R$ & 1252405 & 2 & 8 & 5 & 0 \\
\hline $3 R$ & 1255523 & 2 & 18 & 11 & 0 \\
\hline $3 R$ & 1256048 & 2 & 15 & 7 & 0 \\
\hline $3 R$ & 1261315 & 2 & 12 & 4 & 0 \\
\hline $3 R$ & 1265143 & 2 & 12 & 16 & 0 \\
\hline $3 R$ & 1266966 & 2 & 10 & 6 & 0 \\
\hline $3 R$ & 1270986 & 2 & 10 & 4 & 0 \\
\hline $3 R$ & 1271336 & 2 & 5 & 20 & 0 \\
\hline $3 R$ & 1278168 & 2 & 11 & 8 & 0 \\
\hline $3 R$ & 1279566 & 2 & 22 & 6 & 0 \\
\hline $3 R$ & 1279622 & 2 & 14 & 9 & 0 \\
\hline $3 R$ & 1283893 & 2 & 8 & 21 & 0 \\
\hline $3 R$ & 1289300 & 2 & 10 & 6 & 0 \\
\hline $3 R$ & 1289800 & 2 & 17 & 8 & 0 \\
\hline $3 R$ & 1292381 & 2 & 12 & 12 & 0 \\
\hline $3 R$ & 1295900 & 2 & 26 & 6 & 0 \\
\hline $3 R$ & 1300761 & 2 & 12 & 5 & 0 \\
\hline $3 R$ & 1310118 & 2 & 9 & 4 & 0 \\
\hline $3 R$ & 1313135 & 2 & 9 & 16 & 0 \\
\hline $3 R$ & 1322505 & 2 & 21 & 4 & 0 \\
\hline $3 R$ & 1327881 & 2 & 16 & 4 & 0 \\
\hline $3 R$ & 1329956 & 2 & 13 & 6 & 0 \\
\hline $3 R$ & 1332219 & 2 & 10 & 7 & 0 \\
\hline $3 R$ & 1332514 & 2 & 11 & 6 & 0 \\
\hline $3 R$ & 1337721 & 2 & 27 & 11 & 0 \\
\hline $3 R$ & 1343253 & 2 & 5 & 16 & 0 \\
\hline $3 R$ & 1404043 & 2 & 7 & 6 & 0 \\
\hline $3 R$ & 1409251 & 2 & 6 & 5 & 0 \\
\hline
\end{tabular}




\begin{tabular}{|c|c|c|c|c|c|}
\hline $3 R$ & 1409441 & 2 & 19 & 13 & 0 \\
\hline $3 R$ & 1443727 & 2 & 7 & 6 & 0 \\
\hline $3 R$ & 1470967 & 2 & 18 & 5 & 0 \\
\hline $3 R$ & 1470996 & 2 & 19 & 6 & 0 \\
\hline $3 R$ & 1473363 & 2 & 10 & 12 & 0 \\
\hline $3 R$ & 1485809 & 2 & 4 & 21 & 0 \\
\hline $3 R$ & 1508259 & 2 & 10 & 9 & 0 \\
\hline $3 R$ & 1525849 & 2 & 6 & 13 & 0 \\
\hline $3 R$ & 1531569 & 2 & 6 & 23 & 0 \\
\hline $3 R$ & 1532218 & 2 & 16 & 8 & 0 \\
\hline $3 R$ & 1554782 & 2 & 5 & 6 & 0 \\
\hline $3 R$ & 1556182 & 2 & 14 & 8 & 0 \\
\hline $3 R$ & 1558054 & 2 & 6 & 16 & 0 \\
\hline $3 R$ & 1572112 & 2 & 4 & 18 & 0 \\
\hline $3 R$ & 1583583 & 2 & 12 & 4 & 0 \\
\hline $3 R$ & 1590047 & 2 & 17 & 4 & 0 \\
\hline $3 R$ & 1600481 & 2 & 9 & 5 & 0 \\
\hline $3 R$ & 1603277 & 2 & 7 & 5 & 0 \\
\hline $3 R$ & 1614610 & 2 & 5 & 22 & 0 \\
\hline $3 R$ & 1626527 & 2 & 15 & 8 & 0 \\
\hline $3 R$ & 1694891 & 2 & 5 & 10 & 0 \\
\hline $3 R$ & 1705851 & 2 & 5 & 10 & 0 \\
\hline $3 R$ & 1706794 & 2 & 9 & 11 & 0 \\
\hline $3 R$ & 1710352 & 2 & 18 & 17 & 0 \\
\hline $3 R$ & 1713624 & 2 & 5 & 16 & 0 \\
\hline $3 R$ & 1723217 & 2 & 20 & 5 & 0 \\
\hline $3 R$ & 1728583 & 2 & 8 & 21 & 0 \\
\hline $3 R$ & 1732756 & 2 & 7 & 5 & 0 \\
\hline $3 R$ & 1744683 & 2 & 4 & 16 & 0 \\
\hline $3 R$ & 1744992 & 2 & 17 & 10 & 0 \\
\hline $3 R$ & 1755775 & 2 & 14 & 8 & 0 \\
\hline $3 R$ & 1761400 & 2 & 18 & 5 & 0 \\
\hline $3 R$ & 1761555 & 2 & 15 & 18 & 0 \\
\hline $3 R$ & 1775598 & 2 & 5 & 21 & 0 \\
\hline $3 R$ & 1778767 & 2 & 15 & 5 & 0 \\
\hline $3 R$ & 1779179 & 2 & 14 & 7 & 0 \\
\hline $3 R$ & 1779206 & 2 & 7 & 10 & 0 \\
\hline $3 R$ & 1779252 & 2 & 15 & 8 & 0 \\
\hline $3 R$ & 1791682 & 2 & 11 & 19 & 0 \\
\hline $3 R$ & 1795276 & 2 & 14 & 5 & 0 \\
\hline $3 R$ & 1813345 & 2 & 12 & 5 & 0 \\
\hline $3 R$ & 1830985 & 2 & 13 & 8 & 0 \\
\hline $3 R$ & 1835546 & 2 & 26 & 15 & 0 \\
\hline $3 R$ & 1837267 & 2 & 12 & 5 & 0 \\
\hline $3 R$ & 1839119 & 2 & 11 & 6 & 0 \\
\hline $3 R$ & 1840406 & 2 & 22 & 6 & 0 \\
\hline $3 R$ & 1853984 & 2 & 8 & 5 & 0 \\
\hline $3 R$ & 1854190 & 2 & 23 & 5 & 0 \\
\hline $3 R$ & 1856875 & 2 & 23 & 12 & 0 \\
\hline $3 R$ & 1858291 & 2 & 12 & 4 & 0 \\
\hline $3 R$ & 1864450 & 2 & 7 & 9 & 0 \\
\hline $3 R$ & 1873105 & 2 & 11 & 5 & 0 \\
\hline $3 R$ & 1874128 & 2 & 4 & 11 & 0 \\
\hline
\end{tabular}




$\begin{array}{ll}3 R & 1886063 \\ 3 R & 1889447 \\ 3 R & 1889632 \\ 3 R & 1900677 \\ 3 R & 1900775 \\ 3 R & 1901484 \\ 3 R & 1904115 \\ 3 R & 1913258 \\ 3 R & 1919062 \\ 3 R & 1921527 \\ 3 R & 1922647 \\ 3 R & 1946041 \\ 3 R & 1954322 \\ 3 R & 1956520 \\ 3 R & 1957709 \\ 3 R & 1959779 \\ 3 R & 1974739 \\ 3 R & 1978298 \\ 3 R & 1979507 \\ 3 R & 2016656 \\ 3 R & 2018626 \\ 3 R & 2019879 \\ 3 R & 2026210 \\ 3 R & 2027750 \\ 3 R & 2028515 \\ 3 R & 2030149 \\ 3 R & 2032661 \\ 3 R & 2044973 \\ 3 R & 2052453 \\ 3 R & 2076946 \\ 3 R & 2102627 \\ 3 R & 2110023 \\ 3 R & 2111544 \\ 3 R & 2139791 \\ 3 R & 2142356 \\ 3 R & 2200354 \\ 3 R & 2142500 \\ 3 R & 2143267 \\ 3 R & 2153501 \\ 3 R & 2154002 \\ 3 R & 2162631 \\ 3 R & 2168589 \\ 3 R & 2172617 \\ 3 R & 2181967 \\ 3 R & 2183992 \\ 3 R & 2186513 \\ 3 R & 2195528 \\ 3 R & 2195591 \\ 3 R & 2197966 \\ 3 R & 2214\end{array}$

2

2

2

2

2

2

2

2

2

2

2

2

2

2

2

2

2

2

2

2

2

2

2

2

2

2

2

2

2

2

2

2

2

2

2

2

2

2

2

2

2

2

2

2

2

2

2

2

2

2

2

2

2
17

13

4

23

10

15

13

6

11

11

8

8
20

20

11

14

12

12

7

15

5

5

10

10

5

16

16

18

18

19

10

11

7

16

15

9

11

5

11

11

6

8

14

19

14

17

11

9

10

17

9

15

15

6
$10 \quad 0$

$12 \quad 0$

$6 \quad 0$

$5 \quad 0$

$6 \quad 0$

$4 \quad 0$

130

$4 \quad 0$

$21 \quad 0$

$5 \quad 0$

$7 \quad 0$

$6 \quad 0$

$10 \quad 0$

$5 \quad 0$

$\begin{array}{rr}10 & 0 \\ 4 & 0\end{array}$

$20 \quad 0$

$\begin{array}{ll}7 & 0\end{array}$

$13 \quad 0$

$7 \quad 0$

$8 \quad 0$

60

$11 \quad 0$

$10 \quad 0$

$14-0$

120

$6 \quad 0$

70

6

$6 \quad 0$

$15 \quad 0$

$5 \quad 0$

90

$15 \quad 0$

$5 \quad 0$

$5 \quad 0$

$4 \quad 0$

120

$6 \quad 0$

$16 \quad 0$

$8 \quad 0$

$13 \quad 0$

220

140

$7 \quad 0$

$6 \quad 0$

$6 \quad 0$

12 


\begin{tabular}{|c|c|c|c|c|c|}
\hline $3 R$ & 2240943 & 2 & 4 & 19 & 0 \\
\hline $3 R$ & 2257463 & 2 & 9 & 6 & 0 \\
\hline $3 R$ & 2266228 & 2 & 10 & 11 & 0 \\
\hline $3 R$ & 2266687 & 2 & 12 & 7 & 0 \\
\hline $3 R$ & 2280468 & 2 & 6 & 6 & 0 \\
\hline $3 R$ & 2285882 & 2 & 13 & 8 & 0 \\
\hline $3 R$ & 2305567 & 2 & 6 & 13 & 0 \\
\hline $3 R$ & 2310928 & 2 & 17 & 8 & 0 \\
\hline $3 R$ & 2312427 & 2 & 8 & 8 & 0 \\
\hline $3 R$ & 2314605 & 2 & 13 & 21 & 0 \\
\hline $3 R$ & 2325379 & 2 & 9 & 5 & 0 \\
\hline $3 R$ & 2329283 & 2 & 12 & 5 & 0 \\
\hline $3 R$ & 2344785 & 2 & 4 & 10 & 0 \\
\hline $3 R$ & 2346819 & 2 & 9 & 6 & 0 \\
\hline $3 R$ & 2347028 & 2 & 21 & 7 & 0 \\
\hline $3 R$ & 2350180 & 2 & 8 & 17 & 0 \\
\hline $3 R$ & 2351805 & 2 & 11 & 8 & 0 \\
\hline $3 R$ & 2352794 & 2 & 15 & 7 & 0 \\
\hline $3 R$ & 2357034 & 2 & 9 & 8 & 0 \\
\hline $3 R$ & 2357940 & 2 & 7 & 11 & 0 \\
\hline $3 R$ & 2371101 & 2 & 8 & 11 & 0 \\
\hline $3 R$ & 2372945 & 2 & 7 & 5 & 0 \\
\hline $3 R$ & 2374073 & 2 & 8 & 5 & 0 \\
\hline $3 R$ & 2380759 & 2 & 10 & 6 & 0 \\
\hline $3 R$ & 2393041 & 2 & 4 & 14 & 0 \\
\hline $3 R$ & 2397149 & 2 & 5 & 5 & 0 \\
\hline $3 R$ & 2413300 & 2 & 10 & 7 & 0 \\
\hline $3 R$ & 2424304 & 2 & 7 & 6 & 0 \\
\hline $3 R$ & 2426861 & 2 & 15 & 7 & 0 \\
\hline $3 R$ & 2431700 & 2 & 11 & 9 & 0 \\
\hline $3 R$ & 2453689 & 2 & 7 & 9 & 0 \\
\hline $3 R$ & 2455975 & 2 & 12 & 11 & 0 \\
\hline $3 R$ & 2459534 & 2 & 12 & 7 & 0 \\
\hline $3 R$ & 2466690 & 2 & 8 & 6 & 0 \\
\hline $3 R$ & 2466787 & 2 & 9 & 8 & 0 \\
\hline $3 R$ & 2472706 & 2 & 9 & 4 & 0 \\
\hline $3 R$ & 2478759 & 2 & 15 & 16 & 0 \\
\hline $3 R$ & 2500888 & 2 & 21 & 9 & 0 \\
\hline $3 R$ & 2501362 & 2 & 8 & 6 & 0 \\
\hline $3 R$ & 2537453 & 2 & 8 & 5 & 0 \\
\hline $3 R$ & 2551948 & 2 & 4 & 11 & 0 \\
\hline $3 R$ & 2552409 & 2 & 10 & 5 & 0 \\
\hline $3 R$ & 2568501 & 2 & 11 & 7 & 0 \\
\hline $3 R$ & 2568589 & 2 & 12 & 14 & 0 \\
\hline $3 R$ & 2573613 & 2 & 15 & 11 & 0 \\
\hline $3 R$ & 2577666 & 2 & 11 & 7 & 0 \\
\hline $3 R$ & 2580309 & 2 & 12 & 4 & 0 \\
\hline $3 R$ & 2587170 & 2 & 9 & 4 & 0 \\
\hline $3 R$ & 2587763 & 2 & 13 & 4 & 0 \\
\hline $3 R$ & 2589269 & 2 & 9 & 11 & 0 \\
\hline $3 R$ & 2591530 & 2 & 25 & 9 & 0 \\
\hline $3 R$ & 2603241 & 2 & 9 & 5 & 0 \\
\hline $3 R$ & 2674420 & 2 & 5 & 10 & 0 \\
\hline
\end{tabular}




\begin{tabular}{|c|c|c|c|c|c|}
\hline $3 R$ & 2680255 & 2 & 14 & 16 & 0 \\
\hline $3 R$ & 2683253 & 2 & 9 & 4 & 0 \\
\hline $3 R$ & 2686154 & 2 & 6 & 15 & 0 \\
\hline $3 R$ & 2686169 & 2 & 7 & 8 & 0 \\
\hline $3 R$ & 2688335 & 2 & 10 & 9 & 0 \\
\hline $3 R$ & 2692106 & 2 & 17 & 14 & 0 \\
\hline $3 R$ & 2692506 & 2 & 5 & 5 & 0 \\
\hline $3 R$ & 2693627 & 2 & 17 & 17 & 0 \\
\hline $3 R$ & 2694775 & 2 & 8 & 5 & 0 \\
\hline $3 R$ & 2697159 & 2 & 14 & 9 & 0 \\
\hline $3 R$ & 2697773 & 2 & 11 & 5 & 0 \\
\hline $3 R$ & 2700405 & 2 & 13 & 12 & 0 \\
\hline $3 R$ & 2701957 & 2 & 17 & 9 & 0 \\
\hline $3 R$ & 2702853 & 2 & 15 & 8 & 0 \\
\hline $3 R$ & 2703903 & 2 & 5 & 11 & 0 \\
\hline $3 R$ & 2705887 & 2 & 6 & 8 & 0 \\
\hline $3 R$ & 2707463 & 2 & 8 & 5 & 0 \\
\hline $3 R$ & 2708245 & 2 & 11 & 16 & 0 \\
\hline $3 R$ & 2709113 & 2 & 5 & 10 & 0 \\
\hline $3 R$ & 2710002 & 2 & 5 & 16 & 0 \\
\hline $3 R$ & 2716446 & 2 & 11 & 19 & 0 \\
\hline $3 R$ & 2716689 & 2 & 11 & 15 & 0 \\
\hline $3 R$ & 2720420 & 2 & 14 & 6 & 0 \\
\hline $3 R$ & 2731767 & 2 & 5 & 15 & 0 \\
\hline $3 R$ & 2748004 & 2 & 6 & 19 & 0 \\
\hline $3 R$ & 2748198 & 2 & 5 & 7 & 0 \\
\hline $3 R$ & 2764365 & 2 & 10 & 8 & 0 \\
\hline $3 R$ & 2767568 & 2 & 11 & 7 & 0 \\
\hline $3 R$ & 2770572 & 2 & 7 & 6 & 0 \\
\hline $3 R$ & 2773629 & 2 & 8 & 14 & 0 \\
\hline $3 R$ & 2798978 & 2 & 6 & 7 & 0 \\
\hline $3 R$ & 2805417 & 2 & 9 & 16 & 0 \\
\hline $3 R$ & 2813642 & 2 & 15 & 7 & 0 \\
\hline $3 R$ & 2816089 & 2 & 23 & 5 & 0 \\
\hline $3 R$ & 2832797 & 2 & 17 & 7 & 0 \\
\hline $3 R$ & 2840984 & 2 & 10 & 11 & 0 \\
\hline $3 R$ & 2862070 & 2 & 8 & 5 & 0 \\
\hline $3 R$ & 2862778 & 2 & 18 & 9 & 0 \\
\hline $3 R$ & 2876702 & 2 & 21 & 17 & 0 \\
\hline $3 R$ & 2888602 & 2 & 7 & 8 & 0 \\
\hline $3 R$ & 2891049 & 2 & 17 & 8 & 0 \\
\hline $3 R$ & 2894377 & 2 & 12 & 24 & 0 \\
\hline $3 R$ & 2897201 & 2 & 9 & 15 & 0 \\
\hline $3 R$ & 2907931 & 2 & 15 & 7 & 0 \\
\hline $3 R$ & 2920166 & 2 & 6 & 5 & 0 \\
\hline $3 R$ & 2923506 & 2 & 12 & 5 & 0 \\
\hline $3 R$ & 2965905 & 2 & 6 & 6 & 0 \\
\hline $3 R$ & 2983801 & 2 & 10 & 6 & 0 \\
\hline $3 R$ & 2985886 & 2 & 5 & 20 & 0 \\
\hline $3 R$ & 2988443 & 2 & 8 & 5 & 0 \\
\hline $3 R$ & 2999103 & 2 & 18 & 10 & 0 \\
\hline $3 R$ & 3014006 & 2 & 20 & 7 & 0 \\
\hline $3 R$ & 3036781 & 2 & 6 & 5 & 0 \\
\hline
\end{tabular}




\begin{tabular}{|c|c|c|c|c|c|}
\hline $3 R$ & 3040781 & 2 & 6 & 16 & 0 \\
\hline $3 R$ & 3060824 & 2 & 24 & 18 & 0 \\
\hline $3 R$ & 3061314 & 2 & 7 & 9 & 0 \\
\hline $3 R$ & 3077833 & 2 & 9 & 5 & 0 \\
\hline $3 R$ & 3078885 & 2 & 21 & 11 & 0 \\
\hline $3 R$ & 3097821 & 2 & 4 & 20 & 0 \\
\hline $3 R$ & 3120201 & 2 & 19 & 14 & 0 \\
\hline $3 R$ & 3127377 & 2 & 4 & 12 & 0 \\
\hline $3 R$ & 3136355 & 2 & 12 & 4 & 0 \\
\hline $3 R$ & 3156201 & 2 & 8 & 13 & 0 \\
\hline $3 R$ & 3157582 & 2 & 13 & 16 & 0 \\
\hline $3 R$ & 3161915 & 2 & 4 & 13 & 0 \\
\hline $3 R$ & 3167321 & 2 & 5 & 19 & 0 \\
\hline $3 R$ & 3168325 & 2 & 5 & 11 & 0 \\
\hline $3 R$ & 3195988 & 2 & 12 & 15 & 0 \\
\hline $3 R$ & 3219561 & 2 & 8 & 9 & 0 \\
\hline $3 R$ & 3220248 & 2 & 9 & 6 & 0 \\
\hline $3 R$ & 3247171 & 2 & 20 & 24 & 0 \\
\hline $3 R$ & 3248014 & 2 & 8 & 5 & 0 \\
\hline $3 R$ & 3248074 & 2 & 13 & 10 & 0 \\
\hline $3 R$ & 3248587 & 2 & 17 & 5 & 0 \\
\hline $3 R$ & 3261085 & 2 & 10 & 14 & 0 \\
\hline $3 R$ & 3264805 & 2 & 14 & 4 & 0 \\
\hline $3 R$ & 3265270 & 2 & 4 & 9 & 0 \\
\hline $3 R$ & 3267900 & 2 & 22 & 8 & 0 \\
\hline $3 R$ & 3270952 & 2 & 5 & 26 & 0 \\
\hline $3 R$ & 3271116 & 2 & 7 & 18 & 0 \\
\hline $3 R$ & 3273092 & 2 & 10 & 7 & 0 \\
\hline $3 R$ & 3281209 & 2 & 14 & 14 & 0 \\
\hline $3 R$ & 3290693 & 2 & 24 & 12 & 0 \\
\hline $3 R$ & 3304175 & 2 & 4 & 10 & 0 \\
\hline $3 R$ & 3321515 & 2 & 19 & 9 & 0 \\
\hline $3 R$ & 3324254 & 2 & 13 & 5 & 0 \\
\hline $3 R$ & 3327872 & 2 & 18 & 10 & 0 \\
\hline $3 R$ & 3330983 & 2 & 18 & 10 & 0 \\
\hline $3 R$ & 3334456 & 2 & 20 & 5 & 0 \\
\hline $3 R$ & 3344379 & 2 & 6 & 5 & 0 \\
\hline $3 R$ & 3359421 & 2 & 4 & 16 & 0 \\
\hline $3 R$ & 3366457 & 2 & 13 & 5 & 0 \\
\hline $3 R$ & 3371528 & 2 & 16 & 5 & 0 \\
\hline $3 R$ & 3375894 & 2 & 12 & 6 & 0 \\
\hline $3 R$ & 3377523 & 2 & 16 & 6 & 0 \\
\hline $3 R$ & 3399929 & 2 & 11 & 7 & 0 \\
\hline $3 R$ & 3402373 & 2 & 16 & 6 & 0 \\
\hline $3 R$ & 3409309 & 2 & 14 & 7 & 0 \\
\hline $3 R$ & 3414753 & 2 & 7 & 6 & 0 \\
\hline $3 R$ & 3418663 & 2 & 17 & 4 & 0 \\
\hline $3 R$ & 3422067 & 2 & 7 & 13 & 0 \\
\hline $3 R$ & 3425487 & 2 & 22 & 11 & 0 \\
\hline $3 R$ & 3449061 & 2 & 10 & 24 & 0 \\
\hline $3 R$ & 3453380 & 2 & 8 & 12 & 0 \\
\hline $3 R$ & 3454060 & 2 & 7 & 10 & 0 \\
\hline $3 R$ & 3455980 & 2 & 11 & 9 & 0 \\
\hline
\end{tabular}




\begin{tabular}{|c|c|c|c|c|c|}
\hline $3 R$ & 3461037 & 2 & 14 & 6 & 0 \\
\hline $3 R$ & 3468126 & 2 & 7 & 12 & 0 \\
\hline $3 R$ & 3479633 & 2 & 15 & 4 & 0 \\
\hline $3 R$ & 3482368 & 2 & 10 & 8 & 0 \\
\hline $3 R$ & 3489618 & 2 & 12 & 6 & 0 \\
\hline $3 R$ & 3491733 & 2 & 16 & 12 & 0 \\
\hline $3 R$ & 3492801 & 2 & 8 & 9 & 0 \\
\hline $3 R$ & 3510901 & 2 & 6 & 12 & 0 \\
\hline $3 R$ & 3511833 & 2 & 4 & 16 & 0 \\
\hline $3 R$ & 3543781 & 2 & 4 & 10 & 0 \\
\hline $3 R$ & 3545685 & 2 & 5 & 14 & 0 \\
\hline $3 R$ & 3548769 & 2 & 4 & 17 & 0 \\
\hline $3 R$ & 3550453 & 2 & 5 & 5 & 0 \\
\hline $3 R$ & 3565892 & 2 & 20 & 26 & 0 \\
\hline $3 R$ & 3567883 & 2 & 5 & 15 & 0 \\
\hline $3 R$ & 3601222 & 2 & 7 & 28 & 0 \\
\hline $3 R$ & 3625942 & 2 & 7 & 5 & 0 \\
\hline $3 R$ & 3626131 & 2 & 4 & 10 & 0 \\
\hline $3 R$ & 3669681 & 2 & 6 & 11 & 0 \\
\hline $3 R$ & 3755785 & 2 & 5 & 14 & 0 \\
\hline $3 R$ & 3764340 & 2 & 5 & 9 & 0 \\
\hline $3 R$ & 3779383 & 2 & 11 & 23 & 0 \\
\hline $3 R$ & 3800401 & 2 & 5 & 19 & 0 \\
\hline $3 R$ & 3800599 & 2 & 11 & 7 & 0 \\
\hline $3 R$ & 3803144 & 2 & 5 & 10 & 0 \\
\hline $3 R$ & 3812388 & 2 & 7 & 26 & 0 \\
\hline $3 R$ & 3817677 & 2 & 7 & 23 & 0 \\
\hline $3 R$ & 3856749 & 2 & 6 & 12 & 0 \\
\hline $3 R$ & 3875782 & 2 & 5 & 20 & 0 \\
\hline $3 R$ & 3879632 & 2 & 5 & 10 & 0 \\
\hline $3 R$ & 3883227 & 2 & 12 & 21 & 0 \\
\hline $3 R$ & 3890056 & 2 & 4 & 10 & 0 \\
\hline $3 R$ & 3892478 & 2 & 6 & 9 & 0 \\
\hline $3 R$ & 3896009 & 2 & 5 & 18 & 0 \\
\hline $3 R$ & 3911042 & 2 & 5 & 7 & 0 \\
\hline $3 R$ & 3969785 & 2 & 5 & 8 & 0 \\
\hline $3 R$ & 4010401 & 2 & 6 & 19 & 0 \\
\hline $3 R$ & 4023453 & 2 & 7 & 25 & 0 \\
\hline $3 R$ & 4024466 & 2 & 4 & 18 & 0 \\
\hline $3 R$ & 4047807 & 2 & 11 & 28 & 0 \\
\hline $3 R$ & 4067941 & 2 & 4 & 10 & 0 \\
\hline $3 R$ & 4085358 & 2 & 7 & 19 & 0 \\
\hline $3 R$ & 4103240 & 2 & 6 & 12 & 0 \\
\hline $3 R$ & 4105958 & 2 & 8 & 8 & 0 \\
\hline $3 R$ & 4159743 & 2 & 4 & 14 & 0 \\
\hline $3 R$ & 4212896 & 2 & 10 & 8 & 0 \\
\hline $3 R$ & 4225570 & 2 & 4 & 9 & 0 \\
\hline $3 R$ & 4271394 & 2 & 5 & 11 & 0 \\
\hline $3 R$ & 4277219 & 2 & 5 & 23 & 0 \\
\hline $3 R$ & 4301610 & 2 & 7 & 5 & 0 \\
\hline $3 R$ & 4339796 & 2 & 7 & 16 & 0 \\
\hline $3 R$ & 4411425 & 2 & 6 & 5 & 0 \\
\hline $3 R$ & 4412054 & 2 & 4 & 9 & 0 \\
\hline
\end{tabular}




\begin{tabular}{|c|c|c|c|c|c|}
\hline $3 R$ & 4412250 & 2 & 12 & 8 & 0 \\
\hline $3 R$ & 4419534 & 2 & 7 & 6 & 0 \\
\hline $3 R$ & 4447826 & 2 & 9 & 8 & 0 \\
\hline $3 R$ & 4464541 & 2 & 5 & 28 & 0 \\
\hline $3 R$ & 4472512 & 2 & 15 & 7 & 0 \\
\hline $3 R$ & 4512935 & 2 & 5 & 7 & 0 \\
\hline $3 R$ & 4541707 & 2 & 8 & 6 & 0 \\
\hline $3 R$ & 4550559 & 2 & 6 & 11 & 0 \\
\hline $3 R$ & 4579473 & 2 & 16 & 8 & 0 \\
\hline $3 R$ & 4611088 & 2 & 13 & 8 & 0 \\
\hline $3 R$ & 4615546 & 2 & 5 & 6 & 0 \\
\hline $3 R$ & 4619578 & 2 & 10 & 9 & 0 \\
\hline $3 R$ & 4649529 & 2 & 18 & 6 & 0 \\
\hline $3 R$ & 4661672 & 2 & 5 & 6 & 0 \\
\hline $3 R$ & 4673965 & 2 & 6 & 7 & 0 \\
\hline $3 R$ & 4678694 & 2 & 4 & 9 & 0 \\
\hline $3 R$ & 4703452 & 2 & 21 & 9 & 0 \\
\hline $3 R$ & 4703976 & 2 & 14 & 4 & 0 \\
\hline $3 R$ & 4711570 & 2 & 9 & 8 & 0 \\
\hline $3 R$ & 4718878 & 2 & 15 & 5 & 0 \\
\hline $3 R$ & 4764879 & 2 & 6 & 11 & 0 \\
\hline $3 R$ & 4790759 & 2 & 10 & 7 & 0 \\
\hline $3 R$ & 4793941 & 2 & 6 & 5 & 0 \\
\hline $3 R$ & 4801266 & 2 & 6 & 14 & 0 \\
\hline $3 R$ & 4802435 & 2 & 4 & 9 & 0 \\
\hline $3 R$ & 4804668 & 2 & 5 & 6 & 0 \\
\hline $3 R$ & 4822791 & 2 & 6 & 16 & 0 \\
\hline $3 R$ & 4823096 & 2 & 6 & 5 & 0 \\
\hline $3 R$ & 4850214 & 2 & 4 & 9 & 0 \\
\hline $3 R$ & 4854926 & 2 & 4 & 18 & 0 \\
\hline $3 R$ & 4893117 & 2 & 6 & 13 & 0 \\
\hline $3 R$ & 4895073 & 2 & 4 & 13 & 0 \\
\hline $3 R$ & 4906847 & 2 & 4 & 9 & 0 \\
\hline $3 R$ & 4916894 & 2 & 5 & 11 & 0 \\
\hline $3 R$ & 4917652 & 2 & 8 & 11 & 0 \\
\hline $3 R$ & 4931805 & 2 & 5 & 8 & 0 \\
\hline $3 R$ & 4948445 & 2 & 4 & 13 & 0 \\
\hline $3 R$ & 4951140 & 2 & 5 & 5 & 0 \\
\hline $3 R$ & 4965437 & 2 & 10 & 8 & 0 \\
\hline $3 R$ & 4965931 & 2 & 11 & 10 & 0 \\
\hline $3 R$ & 4985662 & 2 & 6 & 13 & 0 \\
\hline $3 R$ & 4997288 & 2 & 8 & 10 & 0 \\
\hline $3 R$ & 5015330 & 2 & 4 & 14 & 0 \\
\hline $3 R$ & 5038683 & 2 & 4 & 29 & 0 \\
\hline $3 R$ & 5072115 & 2 & 4 & 10 & 0 \\
\hline $3 R$ & 5103676 & 2 & 5 & 23 & 0 \\
\hline $3 R$ & 5129426 & 2 & 12 & 14 & 0 \\
\hline $3 R$ & 5129725 & 2 & 6 & 17 & 0 \\
\hline $3 R$ & 5154499 & 2 & 12 & 16 & 0 \\
\hline $3 R$ & 5154832 & 2 & 5 & 9 & 0 \\
\hline $3 R$ & 5163798 & 2 & 5 & 14 & 0 \\
\hline $3 R$ & 5168265 & 2 & 10 & 6 & 0 \\
\hline $3 R$ & 5204536 & 2 & 5 & 14 & 0 \\
\hline
\end{tabular}




\begin{tabular}{|c|c|c|c|c|c|}
\hline $3 R$ & 5209917 & 2 & 14 & 10 & 0 \\
\hline $3 R$ & 5212912 & 2 & 8 & 20 & 0 \\
\hline $3 R$ & 5301391 & 2 & 15 & 18 & 0 \\
\hline $3 R$ & 5301830 & 2 & 17 & 9 & 0 \\
\hline $3 R$ & 5304728 & 2 & 10 & 10 & 0 \\
\hline $3 R$ & 5306425 & 2 & 9 & 9 & 0 \\
\hline $3 R$ & 5318831 & 2 & 11 & 10 & 0 \\
\hline $3 R$ & 5319871 & 2 & 6 & 6 & 0 \\
\hline $3 R$ & 5368337 & 2 & 4 & 13 & 0 \\
\hline $3 R$ & 5377750 & 2 & 4 & 10 & 0 \\
\hline $3 R$ & 5408269 & 2 & 10 & 10 & 0 \\
\hline $3 R$ & 5410538 & 2 & 6 & 10 & 0 \\
\hline $3 R$ & 5411911 & 2 & 12 & 28 & 0 \\
\hline $3 R$ & 5449070 & 2 & 16 & 8 & 0 \\
\hline $3 R$ & 5452085 & 2 & 6 & 17 & 0 \\
\hline $3 R$ & 5458206 & 2 & 17 & 13 & 0 \\
\hline $3 R$ & 5474860 & 2 & 5 & 15 & 0 \\
\hline $3 R$ & 5652353 & 2 & 5 & 9 & 0 \\
\hline $3 R$ & 5688856 & 2 & 5 & 11 & 0 \\
\hline $3 R$ & 5707066 & 2 & 4 & 14 & 0 \\
\hline $3 R$ & 5722220 & 2 & 5 & 7 & 0 \\
\hline $3 R$ & 5729839 & 2 & 12 & 9 & 0 \\
\hline $3 R$ & 5730289 & 2 & 4 & 12 & 0 \\
\hline $3 R$ & 5742631 & 2 & 8 & 13 & 0 \\
\hline $3 R$ & 5744140 & 2 & 4 & 12 & 0 \\
\hline $3 R$ & 5785443 & 2 & 5 & 12 & 0 \\
\hline $3 R$ & 5819544 & 2 & 13 & 6 & 0 \\
\hline $3 R$ & 5825072 & 2 & 12 & 12 & 0 \\
\hline $3 R$ & 5838019 & 2 & 7 & 16 & 0 \\
\hline $3 R$ & 5866788 & 2 & 8 & 16 & 0 \\
\hline $3 R$ & 5867112 & 2 & 9 & 4 & 0 \\
\hline $3 R$ & 5935087 & 2 & 5 & 10 & 0 \\
\hline $3 R$ & 5948486 & 2 & 16 & 13 & 0 \\
\hline $3 R$ & 5968276 & 2 & 7 & 13 & 0 \\
\hline $3 R$ & 5984374 & 2 & 4 & 22 & 0 \\
\hline $3 R$ & 5985822 & 2 & 7 & 16 & 0 \\
\hline $3 R$ & 5987078 & 2 & 4 & 11 & 0 \\
\hline $3 R$ & 5993723 & 2 & 5 & 11 & 0 \\
\hline $3 R$ & 6014847 & 2 & 4 & 16 & 0 \\
\hline $3 R$ & 6032841 & 2 & 8 & 5 & 0 \\
\hline $3 R$ & 6039042 & 2 & 9 & 5 & 0 \\
\hline $3 R$ & 6050546 & 2 & 12 & 4 & 0 \\
\hline $3 R$ & 6051124 & 2 & 14 & 4 & 0 \\
\hline $3 R$ & 6090694 & 2 & 6 & 5 & 0 \\
\hline $3 R$ & 6152798 & 2 & 11 & 9 & 0 \\
\hline $3 R$ & 6180961 & 2 & 5 & 9 & 0 \\
\hline $3 R$ & 6200914 & 2 & 6 & 20 & 0 \\
\hline $3 R$ & 6219830 & 2 & 9 & 4 & 0 \\
\hline $3 R$ & 6221742 & 2 & 8 & 15 & 0 \\
\hline $3 R$ & 6234301 & 2 & 6 & 9 & 0 \\
\hline $3 R$ & 6288302 & 2 & 4 & 13 & 0 \\
\hline $3 R$ & 6291220 & 2 & 6 & 9 & 0 \\
\hline $3 R$ & 6293287 & 2 & 4 & 9 & 0 \\
\hline
\end{tabular}




\begin{tabular}{|c|c|c|c|c|c|}
\hline $3 R$ & 6297818 & 2 & 11 & 7 & 0 \\
\hline $3 R$ & 6319270 & 2 & 9 & 4 & 0 \\
\hline $3 R$ & 6336221 & 2 & 5 & 8 & 0 \\
\hline $3 R$ & 6353237 & 2 & 4 & 9 & 0 \\
\hline $3 R$ & 6359322 & 2 & 8 & 8 & 0 \\
\hline $3 R$ & 6398702 & 2 & 6 & 6 & 0 \\
\hline $3 R$ & 6422595 & 2 & 5 & 8 & 0 \\
\hline $3 R$ & 6433350 & 2 & 5 & 10 & 0 \\
\hline $3 R$ & 6468346 & 2 & 10 & 6 & 0 \\
\hline $3 R$ & 6471689 & 2 & 9 & 16 & 0 \\
\hline $3 R$ & 6480515 & 2 & 5 & 6 & 0 \\
\hline $3 R$ & 6501696 & 2 & 7 & 13 & 0 \\
\hline $3 R$ & 6507957 & 2 & 15 & 11 & 0 \\
\hline $3 R$ & 6530782 & 2 & 7 & 21 & 0 \\
\hline $3 R$ & 6540074 & 2 & 4 & 10 & 0 \\
\hline $3 R$ & 6562469 & 2 & 17 & 4 & 0 \\
\hline $3 R$ & 6582693 & 2 & 22 & 6 & 0 \\
\hline $3 R$ & 6601520 & 2 & 14 & 5 & 0 \\
\hline $3 R$ & 6616377 & 2 & 15 & 19 & 0 \\
\hline $3 R$ & 6617982 & 2 & 10 & 11 & 0 \\
\hline $3 R$ & 6622501 & 2 & 15 & 10 & 0 \\
\hline $3 R$ & 6628968 & 2 & 10 & 4 & 0 \\
\hline $3 R$ & 6644789 & 2 & 18 & 4 & 0 \\
\hline $3 R$ & 6654705 & 2 & 16 & 6 & 0 \\
\hline $3 R$ & 6662091 & 2 & 4 & 9 & 0 \\
\hline $3 R$ & 6684256 & 2 & 6 & 18 & 0 \\
\hline $3 R$ & 6690776 & 2 & 29 & 4 & 0 \\
\hline $3 R$ & 6691097 & 2 & 6 & 6 & 0 \\
\hline $3 R$ & 6692491 & 2 & 4 & 18 & 0 \\
\hline $3 R$ & 6694701 & 2 & 19 & 11 & 0 \\
\hline $3 R$ & 6696528 & 2 & 13 & 10 & 0 \\
\hline $3 R$ & 6696641 & 2 & 6 & 5 & 0 \\
\hline $3 R$ & 6698339 & 2 & 6 & 5 & 0 \\
\hline $3 R$ & 6720691 & 2 & 6 & 5 & 0 \\
\hline $3 R$ & 6740183 & 2 & 10 & 13 & 0 \\
\hline $3 R$ & 6742003 & 2 & 11 & 24 & 0 \\
\hline $3 R$ & 6757739 & 2 & 11 & 11 & 0 \\
\hline $3 R$ & 6766309 & 2 & 5 & 5 & 0 \\
\hline $3 R$ & 6767031 & 2 & 4 & 9 & 0 \\
\hline $3 R$ & 6771414 & 2 & 6 & 5 & 0 \\
\hline $3 R$ & 6796860 & 2 & 13 & 16 & 0 \\
\hline $3 R$ & 6816763 & 2 & 16 & 9 & 0 \\
\hline $3 R$ & 6836721 & 2 & 6 & 6 & 0 \\
\hline $3 R$ & 6845767 & 2 & 11 & 7 & 0 \\
\hline $3 R$ & 6850975 & 2 & 5 & 14 & 0 \\
\hline $3 R$ & 6856060 & 2 & 9 & 9 & 0 \\
\hline $3 R$ & 6868652 & 2 & 7 & 7 & 0 \\
\hline $3 R$ & 6878495 & 2 & 8 & 10 & 0 \\
\hline $3 R$ & 6889363 & 2 & 8 & 7 & 0 \\
\hline $3 R$ & 6907469 & 2 & 9 & 4 & 0 \\
\hline $3 R$ & 6922869 & 2 & 20 & 8 & 0 \\
\hline $3 R$ & 6928040 & 2 & 14 & 11 & 0 \\
\hline $3 R$ & 6929142 & 2 & 10 & 9 & 0 \\
\hline
\end{tabular}




\begin{tabular}{|c|c|}
\hline $3 R$ & 6934466 \\
\hline $3 R$ & 6942462 \\
\hline $3 R$ & 6955247 \\
\hline $3 R$ & 6958966 \\
\hline $3 R$ & 6968517 \\
\hline $3 R$ & 6973023 \\
\hline $3 R$ & 6990776 \\
\hline $3 R$ & 7072281 \\
\hline $3 R$ & 7075395 \\
\hline $3 R$ & 7081742 \\
\hline $3 R$ & 7082576 \\
\hline $3 R$ & 7089087 \\
\hline $3 R$ & 7114874 \\
\hline $3 R$ & 7137679 \\
\hline $3 R$ & 7139665 \\
\hline $3 R$ & 7143826 \\
\hline $3 R$ & 7154292 \\
\hline $3 R$ & 7166194 \\
\hline $3 R$ & 7192980 \\
\hline $3 R$ & 7278803 \\
\hline $3 R$ & 7307857 \\
\hline $3 R$ & 7312430 \\
\hline $3 R$ & 7321747 \\
\hline $3 R$ & 7355095 \\
\hline $3 R$ & 7360421 \\
\hline $3 R$ & 7388187 \\
\hline $3 R$ & 7411543 \\
\hline $3 R$ & 7471645 \\
\hline $3 R$ & 7492260 \\
\hline $3 R$ & 7497769 \\
\hline $3 R$ & 7503828 \\
\hline $3 R$ & 7505032 \\
\hline $3 R$ & 7506552 \\
\hline $3 R$ & 7508745 \\
\hline $3 R$ & 7535423 \\
\hline $3 R$ & 7538604 \\
\hline $3 R$ & 7542374 \\
\hline $3 R$ & 7544107 \\
\hline $3 R$ & 7576399 \\
\hline $3 R$ & 7579202 \\
\hline $3 R$ & 7592426 \\
\hline $3 R$ & 7641950 \\
\hline $3 R$ & 7657553 \\
\hline $3 R$ & 7674778 \\
\hline $3 R$ & 7686915 \\
\hline $3 R$ & 7701214 \\
\hline $3 R$ & 7710116 \\
\hline $3 R$ & 7710356 \\
\hline $3 R$ & 7711517 \\
\hline $3 R$ & 7719080 \\
\hline $3 R$ & 7727012 \\
\hline $3 R$ & 7739668 \\
\hline $3 R$ & 774 \\
\hline
\end{tabular}

2

2

2

2

2

2

2

2

2

2

2

2

2

2

2

2

2

2

2

2

2

2

2

2

2

2

2

2

2

2

2

2

2

2

2

2

2

2

2

2

2

2

2

2

2

2

2

2

2

2

2

2

2
10

9

13

8

6

8

15

6

8

22

5

11

9

7

$7 \quad 0$

150

$9 \quad 0$

90

150

$8 \quad 0$

$4 \quad 0$

140

$5 \quad 0$

$9 \quad 0$

40

$4 \quad 0$

12

16

13

5

21

6

5

13

7

8

5

20

6

8

14

6

5

10

6

14

4

15

9

11

4

7

5

11

0

0

0

0

0

0

0

0

$$
0
$$

$$
\text { . }
$$

(




$\begin{array}{ll}3 R & 7758897 \\ 3 R & 7761083 \\ 3 R & 7777988 \\ 3 R & 7797822 \\ 3 R & 7800553 \\ 3 R & 7802091 \\ 3 R & 7804045 \\ 3 R & 7804816 \\ 3 R & 7816658 \\ 3 R & 7817712 \\ 3 R & 7818255 \\ 3 R & 7829938 \\ 3 R & 7832709 \\ 3 R & 7851008 \\ 3 R & 7852008 \\ 3 R & 7859271 \\ 3 R & 7864034 \\ 3 R & 7873912 \\ 3 R & 7874179 \\ 3 R & 7880782 \\ 3 R & 7884247 \\ 3 R & 7886141 \\ 3 R & 7892098 \\ 3 R & 7893942 \\ 3 R & 7895897 \\ 3 R & 7899494 \\ 3 R & 7914908 \\ 3 R & 7916005 \\ 3 R & 7927504 \\ 3 R & 7942280 \\ 3 R & 7962663 \\ 3 R & 7970147 \\ 3 R & 7972855 \\ 3 R & 7977720 \\ 3 R & 7979092 \\ 3 R & 7990042 \\ 3 R & 7998787 \\ 3 R & 8003248 \\ 3 R & 8027028 \\ 3 R & 8061395 \\ 3 R & 8070972 \\ 3 R & 8077160 \\ 3 R & 8104344 \\ 3 R & 8108735 \\ 3 R & 8117788 \\ 3 R & 8118643 \\ 3 R & 8131983 \\ 3 R & 8133613 \\ 3 R & 814501 \\ 3 R & \end{array}$

\section{2}

2

2

2

2

2

2

2

2

2

2

2

2

2

2

2

2

2

2

2

2

2

2

2

2

2

2

2

2

2

2

2

2

2

2

2

2

2

2

2

2

2

2

2

2

2

2

2

2

2

2

2

2
17

23

4

8

6

4

6

4
12

9

4

7
12

12

13

7

8

7
8

8
12

7

5

8

17

12

11

4

7

9

16

10

8

4

6

12

5

23

15

19

5

7

12

6

4

9

11

8

7

4

4

11

10

10

10

\section{6}

6

10

21

11

20

6

10

8

9

13

9

7

9

30

6

24

6

7

14

6

7

4

4

19

6

4

9

12

6

15

5

4

13

4

6

4

18

15

19

11

10

7

25

5

20

16

11

4

7

5

8
0

0

0

0

0

0

0

0

0

0

0

0

0

0

0

0

0

0

0

0

0

0

0

0

0

0

0

0

0

0

0

0

0

0

0

0

0

0

0

0

0

0

0

0

0

0

0

0

0 


\begin{tabular}{|c|c|c|c|c|c|}
\hline $3 R$ & 8173405 & 2 & 15 & 5 & 0 \\
\hline $3 R$ & 8173830 & 2 & 12 & 6 & 0 \\
\hline $3 R$ & 8184220 & 2 & 20 & 6 & 0 \\
\hline $3 R$ & 8190543 & 2 & 5 & 17 & 0 \\
\hline $3 R$ & 8190694 & 2 & 6 & 21 & 0 \\
\hline $3 R$ & 8237425 & 2 & 10 & 14 & 0 \\
\hline $3 R$ & 8247252 & 2 & 16 & 12 & 0 \\
\hline $3 R$ & 8249756 & 2 & 15 & 10 & 0 \\
\hline $3 R$ & 8271168 & 2 & 16 & 5 & 0 \\
\hline $3 R$ & 8286537 & 2 & 4 & 20 & 0 \\
\hline $3 R$ & 8343466 & 2 & 4 & 10 & 0 \\
\hline $3 R$ & 8344006 & 2 & 10 & 5 & 0 \\
\hline $3 R$ & 8344644 & 2 & 5 & 11 & 0 \\
\hline $3 R$ & 8364355 & 2 & 7 & 16 & 0 \\
\hline $3 R$ & 8365396 & 2 & 5 & 20 & 0 \\
\hline $3 R$ & 8366867 & 2 & 16 & 4 & 0 \\
\hline $3 R$ & 8378537 & 2 & 9 & 4 & 0 \\
\hline $3 R$ & 8410839 & 2 & 10 & 5 & 0 \\
\hline $3 R$ & 8416163 & 2 & 22 & 8 & 0 \\
\hline $3 R$ & 8426555 & 2 & 4 & 11 & 0 \\
\hline $3 R$ & 8428746 & 2 & 8 & 20 & 0 \\
\hline $3 R$ & 8430466 & 2 & 5 & 12 & 0 \\
\hline $3 R$ & 8435842 & 2 & 5 & 7 & 0 \\
\hline $3 R$ & 8440715 & 2 & 4 & 12 & 0 \\
\hline $3 R$ & 8448444 & 2 & 9 & 9 & 0 \\
\hline $3 R$ & 8472818 & 2 & 14 & 5 & 0 \\
\hline $3 R$ & 8487426 & 2 & 11 & 7 & 0 \\
\hline $3 R$ & 8492844 & 2 & 10 & 11 & 0 \\
\hline $3 R$ & 8493677 & 2 & 29 & 12 & 0 \\
\hline $3 R$ & 8496498 & 2 & 8 & 5 & 0 \\
\hline $3 R$ & 8504484 & 2 & 13 & 6 & 0 \\
\hline $3 R$ & 8523278 & 2 & 8 & 11 & 0 \\
\hline $3 R$ & 8552010 & 2 & 7 & 14 & 0 \\
\hline $3 R$ & 8555418 & 2 & 12 & 4 & 0 \\
\hline $3 R$ & 8560101 & 2 & 5 & 6 & 0 \\
\hline $3 R$ & 8569916 & 2 & 11 & 16 & 0 \\
\hline $3 R$ & 8572212 & 2 & 6 & 10 & 0 \\
\hline $3 R$ & 8576978 & 2 & 4 & 9 & 0 \\
\hline $3 R$ & 8585248 & 2 & 11 & 7 & 0 \\
\hline $3 R$ & 8612781 & 2 & 13 & 4 & 0 \\
\hline $3 R$ & 8613480 & 2 & 15 & 10 & 0 \\
\hline $3 R$ & 8615531 & 2 & 8 & 6 & 0 \\
\hline $3 R$ & 8615668 & 2 & 12 & 7 & 0 \\
\hline $3 R$ & 8616515 & 2 & 11 & 10 & 0 \\
\hline $3 R$ & 8621637 & 2 & 13 & 8 & 0 \\
\hline $3 R$ & 8624787 & 2 & 5 & 5 & 0 \\
\hline $3 R$ & 8627025 & 2 & 5 & 8 & 0 \\
\hline $3 R$ & 8640169 & 2 & 5 & 7 & 0 \\
\hline $3 R$ & 8640204 & 2 & 5 & 12 & 0 \\
\hline $3 R$ & 8645464 & 2 & 6 & 23 & 0 \\
\hline $3 R$ & 8650195 & 2 & 9 & 13 & 0 \\
\hline $3 R$ & 8650899 & 2 & 5 & 9 & 0 \\
\hline $3 R$ & 8656720 & 2 & 8 & 5 & 0 \\
\hline
\end{tabular}




\begin{tabular}{|c|c|c|c|c|c|}
\hline $3 R$ & 8683239 & 2 & 7 & 8 & 0 \\
\hline $3 R$ & 8718793 & 2 & 7 & 11 & 0 \\
\hline $3 R$ & 8720294 & 2 & 9 & 4 & 0 \\
\hline $3 R$ & 8733469 & 2 & 14 & 6 & 0 \\
\hline $3 R$ & 8733474 & 2 & 11 & 7 & 0 \\
\hline $3 R$ & 8752363 & 2 & 6 & 5 & 0 \\
\hline $3 R$ & 8754814 & 2 & 5 & 9 & 0 \\
\hline $3 R$ & 8761849 & 2 & 8 & 5 & 0 \\
\hline $3 R$ & 8763152 & 2 & 14 & 5 & 0 \\
\hline $3 R$ & 8765277 & 2 & 8 & 18 & 0 \\
\hline $3 R$ & 8771798 & 2 & 13 & 7 & 0 \\
\hline $3 R$ & 8776769 & 2 & 10 & 10 & 0 \\
\hline $3 R$ & 8780199 & 2 & 14 & 6 & 0 \\
\hline $3 R$ & 8793684 & 2 & 4 & 15 & 0 \\
\hline $3 R$ & 8809229 & 2 & 12 & 17 & 0 \\
\hline $3 R$ & 8833481 & 2 & 19 & 10 & 0 \\
\hline $3 R$ & 8834623 & 2 & 7 & 9 & 0 \\
\hline $3 R$ & 8839398 & 2 & 10 & 8 & 0 \\
\hline $3 R$ & 8841576 & 2 & 14 & 4 & 0 \\
\hline $3 R$ & 8856881 & 2 & 22 & 10 & 0 \\
\hline $3 R$ & 8858130 & 2 & 6 & 19 & 0 \\
\hline $3 R$ & 8867172 & 2 & 11 & 4 & 0 \\
\hline $3 R$ & 8897602 & 2 & 6 & 13 & 0 \\
\hline $3 R$ & 8897961 & 2 & 11 & 4 & 0 \\
\hline $3 R$ & 8908278 & 2 & 5 & 6 & 0 \\
\hline $3 R$ & 8925850 & 2 & 4 & 17 & 0 \\
\hline $3 R$ & 8994268 & 2 & 11 & 5 & 0 \\
\hline $3 R$ & 9057618 & 2 & 17 & 8 & 0 \\
\hline $3 R$ & 9078324 & 2 & 13 & 5 & 0 \\
\hline $3 R$ & 9081485 & 2 & 5 & 6 & 0 \\
\hline $3 R$ & 9099346 & 2 & 13 & 8 & 0 \\
\hline $3 R$ & 9206033 & 2 & 8 & 5 & 0 \\
\hline $3 R$ & 9236066 & 2 & 9 & 5 & 0 \\
\hline $3 R$ & 9245377 & 2 & 4 & 12 & 0 \\
\hline $3 R$ & 9258216 & 2 & 11 & 4 & 0 \\
\hline $3 R$ & 9277109 & 2 & 19 & 11 & 0 \\
\hline $3 R$ & 9278385 & 2 & 9 & 6 & 0 \\
\hline $3 R$ & 9285145 & 2 & 11 & 7 & 0 \\
\hline $3 R$ & 9285488 & 2 & 20 & 29 & 0 \\
\hline $3 R$ & 9296906 & 2 & 6 & 7 & 0 \\
\hline $3 R$ & 9306636 & 2 & 6 & 13 & 0 \\
\hline $3 R$ & 9319162 & 2 & 5 & 23 & 0 \\
\hline $3 R$ & 9324313 & 2 & 5 & 7 & 0 \\
\hline $3 R$ & 9324924 & 2 & 10 & 5 & 0 \\
\hline $3 R$ & 9381418 & 2 & 9 & 6 & 0 \\
\hline $3 R$ & 9385737 & 2 & 10 & 9 & 0 \\
\hline $3 R$ & 9388155 & 2 & 7 & 18 & 0 \\
\hline $3 R$ & 9419189 & 2 & 11 & 5 & 0 \\
\hline $3 R$ & 9502358 & 2 & 8 & 7 & 0 \\
\hline $3 R$ & 9548808 & 2 & 6 & 9 & 0 \\
\hline $3 R$ & 9585079 & 2 & 10 & 10 & 0 \\
\hline $3 R$ & 9621450 & 2 & 8 & 5 & 0 \\
\hline $3 R$ & 9623482 & 2 & 6 & 10 & 0 \\
\hline
\end{tabular}




\begin{tabular}{|c|c|c|c|c|c|}
\hline $3 R$ & 9646218 & 2 & 13 & 7 & 0 \\
\hline $3 R$ & 9650789 & 2 & 9 & 5 & 0 \\
\hline $3 R$ & 9650796 & 2 & 9 & 4 & 0 \\
\hline $3 R$ & 9675873 & 2 & 7 & 5 & 0 \\
\hline $3 R$ & 9679941 & 2 & 7 & 8 & 0 \\
\hline $3 R$ & 9716357 & 2 & 5 & 8 & 0 \\
\hline $3 R$ & 9716538 & 2 & 5 & 6 & 0 \\
\hline $3 R$ & 9718901 & 2 & 4 & 23 & 0 \\
\hline $3 R$ & 9734074 & 2 & 16 & 7 & 0 \\
\hline $3 R$ & 9735938 & 2 & 13 & 7 & 0 \\
\hline $3 R$ & 9745579 & 2 & 12 & 12 & 0 \\
\hline $3 R$ & 9770520 & 2 & 5 & 5 & 0 \\
\hline $3 R$ & 9776903 & 2 & 12 & 6 & 0 \\
\hline $3 R$ & 9794542 & 2 & 13 & 5 & 0 \\
\hline $3 R$ & 9835562 & 2 & 9 & 7 & 0 \\
\hline $3 R$ & 9861379 & 2 & 4 & 21 & 0 \\
\hline $3 R$ & 10130589 & 2 & 19 & 4 & 0 \\
\hline $3 R$ & 10137871 & 2 & 5 & 9 & 0 \\
\hline $3 R$ & 11390609 & 2 & 9 & 4 & 0 \\
\hline $3 R$ & 11631542 & 2 & 5 & 9 & 0 \\
\hline $3 R$ & 11671233 & 2 & 4 & 9 & 0 \\
\hline $3 R$ & 12784541 & 2 & 6 & 8 & 0 \\
\hline $3 R$ & 12842110 & 2 & 8 & 10 & 0 \\
\hline $3 R$ & 12843918 & 2 & 4 & 9 & 0 \\
\hline $3 R$ & 12887849 & 2 & 5 & 8 & 0 \\
\hline $3 R$ & 13066472 & 2 & 4 & 15 & 0 \\
\hline $3 R$ & 13093562 & 2 & 5 & 5 & 0 \\
\hline $3 R$ & 13211899 & 2 & 5 & 9 & 0 \\
\hline $3 R$ & 13234914 & 2 & 5 & 6 & 0 \\
\hline $3 R$ & 13308882 & 2 & 6 & 7 & 0 \\
\hline $3 R$ & 13426412 & 2 & 12 & 5 & 0 \\
\hline $3 R$ & 13593061 & 2 & 6 & 9 & 0 \\
\hline $3 R$ & 13696430 & 2 & 5 & 6 & 0 \\
\hline $3 R$ & 13734327 & 2 & 7 & 17 & 0 \\
\hline $3 R$ & 14037551 & 2 & 8 & 9 & 0 \\
\hline $3 R$ & 14138534 & 2 & 11 & 6 & 0 \\
\hline $3 R$ & 14339293 & 2 & 4 & 12 & 0 \\
\hline $3 R$ & 14357650 & 2 & 4 & 11 & 0 \\
\hline $3 R$ & 14377140 & 2 & 6 & 13 & 0 \\
\hline $3 R$ & 14381191 & 2 & 8 & 10 & 0 \\
\hline $3 R$ & 14413418 & 2 & 6 & 5 & 0 \\
\hline $3 R$ & 14415032 & 2 & 10 & 16 & 0 \\
\hline $3 R$ & 14488178 & 2 & 7 & 10 & 0 \\
\hline $3 R$ & 14499725 & 2 & 4 & 11 & 0 \\
\hline $3 R$ & 14504667 & 2 & 5 & 5 & 0 \\
\hline $3 R$ & 14523836 & 2 & 6 & 12 & 0 \\
\hline $3 R$ & 14945211 & 2 & 5 & 7 & 0 \\
\hline $3 R$ & 15061093 & 2 & 9 & 4 & 0 \\
\hline $3 R$ & 15141735 & 2 & 5 & 6 & 0 \\
\hline $3 R$ & 15344440 & 2 & 6 & 5 & 0 \\
\hline $3 R$ & 15802873 & 2 & 17 & 4 & 0 \\
\hline $3 R$ & 16092053 & 2 & 5 & 10 & 0 \\
\hline $3 R$ & 16173175 & 2 & 5 & 7 & 0 \\
\hline
\end{tabular}




\begin{tabular}{|c|c|c|c|c|c|}
\hline $3 R$ & 16692895 & 2 & 5 & 5 & 0 \\
\hline $3 R$ & 17201269 & 2 & 19 & 4 & 0 \\
\hline $3 R$ & 17420865 & 2 & 4 & 9 & 0 \\
\hline $3 R$ & 17782444 & 2 & 4 & 9 & 0 \\
\hline $3 R$ & 20636763 & 2 & 10 & 7 & 0 \\
\hline $3 R$ & 20691742 & 2 & 9 & 6 & 0 \\
\hline $3 R$ & 20703001 & 2 & 6 & 7 & 0 \\
\hline $3 R$ & 20738747 & 2 & 5 & 10 & 0 \\
\hline $3 R$ & 20747095 & 2 & 12 & 5 & 0 \\
\hline $3 R$ & 20930994 & 2 & 6 & 7 & 0 \\
\hline $3 R$ & 20931458 & 2 & 6 & 5 & 0 \\
\hline $3 R$ & 20932299 & 2 & 9 & 4 & 0 \\
\hline $3 R$ & 20957331 & 2 & 9 & 4 & 0 \\
\hline $3 R$ & 20993247 & 2 & 5 & 5 & 0 \\
\hline $3 R$ & 20997255 & 2 & 8 & 13 & 0 \\
\hline $3 R$ & 21013830 & 2 & 9 & 4 & 0 \\
\hline $3 R$ & 21021456 & 2 & 6 & 7 & 0 \\
\hline $3 R$ & 21034741 & 2 & 5 & 7 & 0 \\
\hline $3 R$ & 21035237 & 2 & 10 & 6 & 0 \\
\hline $3 R$ & 21040529 & 2 & 9 & 5 & 0 \\
\hline $3 R$ & 21045802 & 2 & 13 & 6 & 0 \\
\hline $3 R$ & 21072864 & 2 & 8 & 5 & 0 \\
\hline $3 R$ & 21094509 & 2 & 9 & 4 & 0 \\
\hline $3 R$ & 21096564 & 2 & 6 & 10 & 0 \\
\hline $3 R$ & 21097207 & 2 & 4 & 9 & 0 \\
\hline $3 R$ & 21118407 & 2 & 7 & 6 & 0 \\
\hline $3 R$ & 21122720 & 2 & 10 & 4 & 0 \\
\hline $3 R$ & 21127478 & 2 & 7 & 10 & 0 \\
\hline $3 R$ & 21127568 & 2 & 12 & 5 & 0 \\
\hline $3 R$ & 21131466 & 2 & 11 & 5 & 0 \\
\hline $3 R$ & 21278955 & 2 & 6 & 8 & 0 \\
\hline $3 R$ & 21354324 & 2 & 8 & 8 & 0 \\
\hline $3 R$ & 21373852 & 2 & 5 & 5 & 0 \\
\hline $3 R$ & 21429727 & 2 & 13 & 4 & 0 \\
\hline $3 R$ & 21453004 & 2 & 12 & 4 & 0 \\
\hline $3 R$ & 21455946 & 2 & 13 & 7 & 0 \\
\hline $3 R$ & 21527432 & 2 & 12 & 8 & 0 \\
\hline $3 R$ & 21567789 & 2 & 6 & 5 & 0 \\
\hline $3 R$ & 21567827 & 2 & 9 & 7 & 0 \\
\hline $3 R$ & 21643061 & 2 & 8 & 5 & 0 \\
\hline $3 R$ & 21680890 & 2 & 9 & 15 & 0 \\
\hline $3 R$ & 21724036 & 2 & 5 & 6 & 0 \\
\hline $3 R$ & 21740609 & 2 & 6 & 6 & 0 \\
\hline $3 R$ & 21747063 & 2 & 7 & 5 & 0 \\
\hline $3 R$ & 21910389 & 2 & 5 & 15 & 0 \\
\hline $3 R$ & 21925737 & 2 & 5 & 11 & 0 \\
\hline $3 R$ & 21977976 & 2 & 7 & 6 & 0 \\
\hline $3 R$ & 21995673 & 2 & 5 & 7 & 0 \\
\hline $3 R$ & 22017318 & 2 & 8 & 8 & 0 \\
\hline $3 R$ & 22026020 & 2 & 15 & 9 & 0 \\
\hline $3 R$ & 22037414 & 2 & 5 & 10 & 0 \\
\hline $3 R$ & 22091088 & 2 & 6 & 6 & 0 \\
\hline $3 R$ & 22157975 & 2 & 4 & 9 & 0 \\
\hline
\end{tabular}


$3 R$

$3 R$

$3 R$

$3 R$

$3 R$

$3 R$

$3 R$

$3 R$

$3 R$

$3 R$

$3 R$

$3 R$

$3 R$

$3 R$

$3 R$

$3 R$

$3 R$

$3 R$

$3 R$

$3 R$

$3 R$

$3 R$

$X$

$x$

$x$

$x$

$x$

$x$

$x$

$x$

$x$

$x$

$x$

$x$

$x$

$x$

$x$

$x$

$x$

$x$

$x$

$x$

$x$

$x$

$x$

$x$

$x$

$x$

$x$

$x$

$x$

$x$

$x$
22175880

22187957

22759044

22899508

22900563

22911498

22927276

22959922

23230447

23299380

23425352

23459094

23463972

23490478

23505408

23608768

23624647

24116080

24438982

24580256

24681117

24845764

36653

79749

88322

97518

142650

146867

153547

156117

178527

179450

202395

203434

206104

332653

344142

367036

385294

391919

483177

487662

497489

498560

501330

502553

503864

504181

507079

511485

511617

514945

515344
2

2

2

2

2

2

2

2

2

2

2

2

2

2

2

2

2

2

2

2

2

2

2

2

2

2

2

2

2

2

2

2

2

2

2

2

2

2

2

2

2

2

2

2

2

2

2

2

2

2

2

2

2

6
9
9
9
5

14

16

7

7

6

5

6

9
17

4

11

5

7

5

5

7

8

6

4

9

12

16

4

5

12

11

9

14

14

5

13

5

8

12

12

6

6

9

13

24

4

24

10

21

31

10

21
5

$7 \quad 0$

$5 \quad 0$

$9 \quad 0$

7

6

5

$7 \quad 0$

$6 \quad 0$

$10 \quad 0$

$\begin{array}{ll}7 & 0 \\ 7 & 0\end{array}$

$7 \quad 0$

$\begin{array}{ll}6 & 0 \\ 4 & 0\end{array}$

$9 \quad 0$

$6 \quad 0$

$8 \quad 0$

$8 \quad 0$

$5 \quad 0$

$7 \quad 0$

$\begin{array}{ll}7 & 0\end{array}$

$\begin{array}{ll}8 & 0 \\ 9 & 0\end{array}$

220

$6 \quad 0$

$\begin{array}{ll}4 & 0 \\ 2 & 0\end{array}$

100

$15 \quad 0$

$5 \quad 0$

$13 \quad 0$

$16 \quad 0$

210

18

130

$6 \quad 0$

$16 \quad 0$

130

$6 \quad 0$

$6 \quad 0$

240

$8 \quad 0$

$7 \quad 0$

$13 \quad 0$

120

40

$6 \quad 0$

$10 \quad 0$

130

$15 \quad 0$

50

$6 \quad 0$ 


\begin{tabular}{|c|c|}
\hline$x$ & 518765 \\
\hline$X$ & 518884 \\
\hline$x$ & 521738 \\
\hline$x$ & 521808 \\
\hline$x$ & 522066 \\
\hline$x$ & 522240 \\
\hline $\mathrm{X}$ & 524614 \\
\hline $\mathrm{X}$ & 524854 \\
\hline$x$ & 525044 \\
\hline$x$ & 525143 \\
\hline$x$ & 527808 \\
\hline$x$ & 528142 \\
\hline$x$ & 528657 \\
\hline$x$ & 534697 \\
\hline$x$ & 537980 \\
\hline$x$ & 556614 \\
\hline$x$ & 558781 \\
\hline$x$ & 572750 \\
\hline$x$ & 576737 \\
\hline $\mathrm{X}$ & 578546 \\
\hline$x$ & 590030 \\
\hline$x$ & 590476 \\
\hline$x$ & 601434 \\
\hline$x$ & 601944 \\
\hline$x$ & 602144 \\
\hline$x$ & 602341 \\
\hline$x$ & 602787 \\
\hline$X$ & 610972 \\
\hline$x$ & 614767 \\
\hline$x$ & 615697 \\
\hline$x$ & 617264 \\
\hline $\mathrm{X}$ & 619862 \\
\hline$x$ & 628150 \\
\hline$x$ & 633458 \\
\hline$x$ & 638205 \\
\hline$x$ & 642755 \\
\hline$x$ & 660427 \\
\hline$x$ & 662012 \\
\hline$x$ & 665209 \\
\hline$x$ & 668614 \\
\hline$x$ & 668855 \\
\hline$x$ & 669238 \\
\hline$x$ & 670097 \\
\hline$x$ & 670263 \\
\hline$x$ & 674368 \\
\hline$x$ & 679535 \\
\hline$x$ & 686968 \\
\hline$x$ & 687475 \\
\hline$x$ & 689156 \\
\hline$x$ & 691879 \\
\hline$x$ & 692256 \\
\hline$x$ & 695073 \\
\hline$X$ & 701837 \\
\hline
\end{tabular}

\begin{tabular}{|c|c|c|}
\hline 25 & 8 & 0 \\
\hline 22 & 13 & 0 \\
\hline 10 & 4 & 0 \\
\hline 25 & 16 & 0 \\
\hline 11 & 11 & 0 \\
\hline 10 & 5 & 0 \\
\hline 12 & 11 & 0 \\
\hline 37 & 14 & 0 \\
\hline 21 & 11 & 0 \\
\hline 26 & 22 & 0 \\
\hline 12 & 7 & 0 \\
\hline 21 & 16 & 0 \\
\hline 18 & 7 & 0 \\
\hline 15 & 4 & 0 \\
\hline 4 & 14 & 0 \\
\hline 18 & 14 & 0 \\
\hline 9 & 25 & 0 \\
\hline 19 & 11 & 0 \\
\hline 4 & 11 & 0 \\
\hline 20 & 9 & 0 \\
\hline 5 & 18 & 0 \\
\hline 9 & 14 & 0 \\
\hline 6 & 10 & 0 \\
\hline 11 & 10 & 0 \\
\hline 23 & 4 & 0 \\
\hline 25 & 6 & 0 \\
\hline 18 & 15 & 0 \\
\hline 21 & 10 & 0 \\
\hline 15 & 8 & 0 \\
\hline 11 & 7 & 0 \\
\hline 15 & 11 & 0 \\
\hline 6 & 9 & 0 \\
\hline 19 & 23 & 0 \\
\hline 18 & 17 & 0 \\
\hline 24 & 29 & 0 \\
\hline 32 & 20 & 0 \\
\hline 11 & 7 & 0 \\
\hline 25 & 9 & 0 \\
\hline 21 & 13 & 0 \\
\hline 22 & 14 & 0 \\
\hline 10 & 5 & 0 \\
\hline 21 & 8 & 0 \\
\hline 20 & 6 & 0 \\
\hline 17 & 4 & 0 \\
\hline 15 & 7 & 0 \\
\hline 27 & 13 & 0 \\
\hline 22 & 19 & 0 \\
\hline 4 & 22 & 0 \\
\hline 18 & 4 & 0 \\
\hline 17 & 19 & 0 \\
\hline 4 & 22 & 0 \\
\hline 13 & 6 & 0 \\
\hline 9 & 24 & 0 \\
\hline
\end{tabular}




\begin{tabular}{|c|c|}
\hline$x$ & 715838 \\
\hline$x$ & 717437 \\
\hline$X$ & 718532 \\
\hline$x$ & 718644 \\
\hline$x$ & 731561 \\
\hline$x$ & 731621 \\
\hline$x$ & 731735 \\
\hline $\mathrm{X}$ & 736751 \\
\hline$x$ & 749036 \\
\hline$x$ & 750996 \\
\hline$x$ & 751068 \\
\hline$x$ & 753939 \\
\hline$x$ & 754798 \\
\hline$x$ & 756219 \\
\hline$x$ & 756716 \\
\hline$x$ & 757844 \\
\hline$x$ & 765233 \\
\hline$x$ & 767277 \\
\hline$x$ & 767894 \\
\hline$x$ & 767937 \\
\hline$x$ & 767986 \\
\hline$x$ & 768222 \\
\hline$x$ & 769576 \\
\hline$x$ & 770229 \\
\hline$x$ & 770445 \\
\hline$x$ & 770532 \\
\hline$x$ & 771947 \\
\hline$x$ & 779799 \\
\hline$x$ & 781273 \\
\hline$x$ & 785807 \\
\hline$x$ & 786005 \\
\hline$x$ & 786522 \\
\hline$x$ & 789962 \\
\hline$x$ & 790401 \\
\hline$x$ & 791219 \\
\hline$x$ & 794372 \\
\hline$x$ & 796305 \\
\hline$x$ & 796633 \\
\hline$x$ & 803810 \\
\hline$x$ & 806724 \\
\hline$x$ & 815515 \\
\hline$x$ & 817883 \\
\hline$x$ & 821204 \\
\hline$x$ & 821989 \\
\hline$x$ & 822011 \\
\hline$x$ & 822064 \\
\hline$x$ & 823788 \\
\hline$x$ & 824785 \\
\hline$x$ & 826913 \\
\hline$x$ & 831857 \\
\hline$x$ & 843377 \\
\hline$x$ & 849369 \\
\hline$x$ & 8578 \\
\hline
\end{tabular}

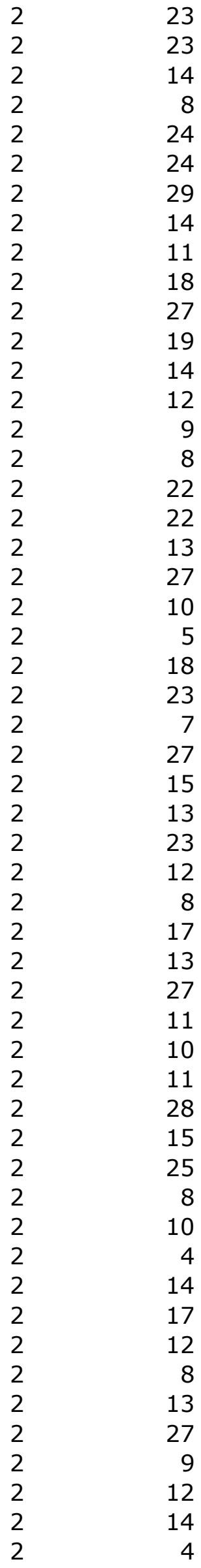




\begin{tabular}{|c|c|}
\hline$x$ & 859623 \\
\hline$x$ & 860468 \\
\hline$x$ & 868078 \\
\hline$x$ & 874281 \\
\hline$x$ & 876541 \\
\hline$x$ & 876645 \\
\hline$x$ & 876785 \\
\hline$x$ & 877238 \\
\hline$x$ & 883644 \\
\hline$x$ & 889209 \\
\hline$x$ & 890845 \\
\hline$X$ & 892612 \\
\hline$x$ & 893806 \\
\hline$x$ & 895951 \\
\hline$x$ & 897788 \\
\hline$x$ & 902523 \\
\hline$x$ & 902792 \\
\hline$x$ & 902913 \\
\hline$x$ & 903038 \\
\hline$x$ & 904081 \\
\hline$x$ & 906824 \\
\hline$x$ & 907331 \\
\hline$x$ & 908189 \\
\hline$x$ & 909249 \\
\hline$x$ & 914893 \\
\hline$x$ & 916150 \\
\hline$x$ & 917886 \\
\hline$x$ & 919811 \\
\hline$x$ & 922020 \\
\hline$x$ & 924391 \\
\hline$x$ & 940904 \\
\hline$x$ & 941409 \\
\hline$x$ & 946142 \\
\hline$x$ & 947207 \\
\hline$x$ & 952443 \\
\hline$x$ & 954751 \\
\hline$X$ & 968950 \\
\hline$x$ & 974492 \\
\hline$x$ & 980364 \\
\hline$x$ & 990109 \\
\hline$x$ & 992260 \\
\hline$x$ & 994785 \\
\hline$x$ & 995094 \\
\hline$x$ & 996027 \\
\hline$x$ & 996289 \\
\hline$x$ & 996535 \\
\hline$x$ & 996945 \\
\hline$x$ & 997222 \\
\hline$x$ & 997664 \\
\hline$x$ & 997741 \\
\hline$x$ & 997840 \\
\hline$x$ & 998802 \\
\hline$x$ & 1003 \\
\hline
\end{tabular}

\begin{tabular}{|c|c|}
\hline \multirow{2}{*}{1} & 21 \\
\hline & 14 \\
\hline 6 & 24 \\
\hline 14 & 8 \\
\hline 6 & 8 \\
\hline 4 & 19 \\
\hline 4 & 12 \\
\hline 4 & 12 \\
\hline 6 & 8 \\
\hline 5 & 15 \\
\hline 9 & 7 \\
\hline 12 & 5 \\
\hline 16 & 5 \\
\hline 28 & 4 \\
\hline 5 & 17 \\
\hline 6 & 12 \\
\hline 4 & 17 \\
\hline 5 & 16 \\
\hline 4 & 16 \\
\hline 11 & 15 \\
\hline 10 & 21 \\
\hline 6 & 13 \\
\hline 7 & 13 \\
\hline 10 & 16 \\
\hline 3 & 34 \\
\hline 4 & 13 \\
\hline 10 & 13 \\
\hline 9 & 20 \\
\hline 7 & 19 \\
\hline 10 & 19 \\
\hline 7 & 21 \\
\hline 9 & 13 \\
\hline 6 & 12 \\
\hline 20 & 13 \\
\hline 5 & 14 \\
\hline 10 & 11 \\
\hline 12 & 19 \\
\hline 6 & 28 \\
\hline 16 & 22 \\
\hline 12 & 18 \\
\hline 17 & 19 \\
\hline 7 & 10 \\
\hline 5 & 14 \\
\hline 9 & 14 \\
\hline 9 & 19 \\
\hline 7 & 11 \\
\hline 10 & 11 \\
\hline 13 & 24 \\
\hline 11 & 23 \\
\hline 13 & 22 \\
\hline 15 & 23 \\
\hline 9 & 19 \\
\hline 6 & 3 \\
\hline
\end{tabular}




\begin{tabular}{|c|c|c|c|c|c|}
\hline$x$ & 1003539 & 2 & 18 & 12 & 0 \\
\hline$x$ & 1007067 & 2 & 6 & 18 & 0 \\
\hline$x$ & 1021129 & 2 & 14 & 4 & 0 \\
\hline$x$ & 1021393 & 2 & 9 & 10 & 0 \\
\hline$x$ & 1021437 & 2 & 6 & 14 & 0 \\
\hline$x$ & 1035573 & 2 & 14 & 10 & 0 \\
\hline$x$ & 1045669 & 2 & 4 & 10 & 0 \\
\hline$x$ & 1055445 & 2 & 24 & 22 & 0 \\
\hline$x$ & 1062336 & 2 & 12 & 26 & 0 \\
\hline$x$ & 1088117 & 2 & 8 & 17 & 0 \\
\hline$x$ & 1094788 & 2 & 6 & 9 & 0 \\
\hline$x$ & 1096849 & 2 & 5 & 13 & 0 \\
\hline$x$ & 1097844 & 2 & 11 & 18 & 0 \\
\hline$x$ & 1099851 & 2 & 4 & 13 & 0 \\
\hline$x$ & 1100250 & 2 & 8 & 26 & 0 \\
\hline$x$ & 1104881 & 2 & 5 & 5 & 0 \\
\hline$X$ & 1116694 & 2 & 8 & 19 & 0 \\
\hline$x$ & 1118921 & 2 & 4 & 10 & 0 \\
\hline$x$ & 1120421 & 2 & 9 & 17 & 0 \\
\hline$x$ & 1123744 & 2 & 8 & 17 & 0 \\
\hline$x$ & 1140734 & 2 & 12 & 12 & 0 \\
\hline$x$ & 1144193 & 2 & 7 & 18 & 0 \\
\hline$x$ & 1144350 & 2 & 14 & 9 & 0 \\
\hline$x$ & 1146443 & 2 & 7 & 8 & 0 \\
\hline$x$ & 1147150 & 2 & 5 & 21 & 0 \\
\hline$x$ & 1149009 & 2 & 8 & 10 & 0 \\
\hline$x$ & 1149057 & 2 & 14 & 23 & 0 \\
\hline$x$ & 1150139 & 2 & 7 & 10 & 0 \\
\hline$x$ & 1150587 & 2 & 11 & 21 & 0 \\
\hline$x$ & 1150974 & 2 & 15 & 21 & 0 \\
\hline$x$ & 1155922 & 2 & 11 & 24 & 0 \\
\hline$x$ & 1157570 & 2 & 4 & 22 & 0 \\
\hline$x$ & 1157661 & 2 & 5 & 16 & 0 \\
\hline$x$ & 1157931 & 2 & 7 & 9 & 0 \\
\hline$x$ & 1159617 & 2 & 27 & 25 & 0 \\
\hline$x$ & 1160748 & 2 & 5 & 10 & 0 \\
\hline$x$ & 1160943 & 2 & 15 & 22 & 0 \\
\hline$x$ & 1161079 & 2 & 11 & 11 & 0 \\
\hline$x$ & 1161739 & 2 & 9 & 16 & 0 \\
\hline$x$ & 1164140 & 2 & 10 & 8 & 0 \\
\hline$x$ & 1168902 & 2 & 8 & 12 & 0 \\
\hline$x$ & 1170233 & 2 & 8 & 21 & 0 \\
\hline$x$ & 1174278 & 2 & 8 & 10 & 0 \\
\hline$x$ & 1177488 & 2 & 13 & 6 & 0 \\
\hline$x$ & 1183534 & 2 & 9 & 15 & 0 \\
\hline$x$ & 1189782 & 2 & 17 & 17 & 0 \\
\hline$x$ & 1189916 & 2 & 11 & 41 & 0 \\
\hline$x$ & 1189931 & 2 & 5 & 40 & 0 \\
\hline$x$ & 1192950 & 2 & 5 & 10 & 0 \\
\hline$x$ & 1199582 & 2 & 5 & 8 & 0 \\
\hline$x$ & 1207126 & 2 & 5 & 11 & 0 \\
\hline$x$ & 1213405 & 2 & 7 & 15 & 0 \\
\hline$x$ & 1218809 & 2 & 16 & 10 & 0 \\
\hline
\end{tabular}




\begin{tabular}{|c|c|}
\hline$x$ & 1221603 \\
\hline$x$ & 1230196 \\
\hline$x$ & 1237093 \\
\hline$x$ & 1244056 \\
\hline$x$ & 1272269 \\
\hline$x$ & 1286120 \\
\hline$x$ & 1293664 \\
\hline$x$ & 1304064 \\
\hline$x$ & 1307427 \\
\hline$x$ & 1309548 \\
\hline$x$ & 1309878 \\
\hline$x$ & 1311119 \\
\hline$x$ & 1312438 \\
\hline$x$ & 1312645 \\
\hline$x$ & 1315496 \\
\hline$x$ & 1319760 \\
\hline$x$ & 1322591 \\
\hline$x$ & 1324272 \\
\hline$x$ & 1326715 \\
\hline$x$ & 1327240 \\
\hline$x$ & 1328062 \\
\hline$x$ & 1328659 \\
\hline$x$ & 1328825 \\
\hline$x$ & 1333940 \\
\hline$x$ & 1335090 \\
\hline$x$ & 1335212 \\
\hline$x$ & 1335668 \\
\hline$x$ & 1336024 \\
\hline$x$ & 1336774 \\
\hline$x$ & 1336906 \\
\hline$x$ & 1338640 \\
\hline$x$ & 1338741 \\
\hline$x$ & 1339495 \\
\hline$X$ & 1339745 \\
\hline$x$ & 1339842 \\
\hline$x$ & 1340293 \\
\hline$x$ & 1340403 \\
\hline$x$ & 1340492 \\
\hline$x$ & 1340961 \\
\hline$x$ & 1340982 \\
\hline$x$ & 1341184 \\
\hline$x$ & 1341280 \\
\hline$x$ & 1341365 \\
\hline$x$ & 1341408 \\
\hline$x$ & 1341783 \\
\hline$X$ & 1341924 \\
\hline$x$ & 1342110 \\
\hline$x$ & 1342242 \\
\hline$x$ & 1342401 \\
\hline$x$ & 1342680 \\
\hline$X$ & 1342998 \\
\hline$X$ & 1343094 \\
\hline$x$ & 1343 \\
\hline
\end{tabular}

2

2

2

2

2

2

2

2

2

2

2

2

2

2

2

2

2

2

2

2

2

2

2

2

2

2

2

2

2

2

2

2

2

2

2

2

2

2

2

2

2

2

2

2

2

2

2

2

2

2

2

2

2
18

5

11

14

13

14

14

22

4

6

7

7

13

11

14

4

7

9
15

8

5

5

4

8

11

8

19

8

5
10

10
14

16

8

7

6

7

17

4

8

4

6

7

8

8

7

6

7

6

21

7

5

19

5
25

21

22

14

18

32

21

16

24

12

20

20

34

13

15

15

13

28

13

20

11

12

27

19

19

26

24

23

15

19

22

16

22

31

26

25

10

26

22

12

24

27

29

9

29

19

5

31

12

14

32

15
0

0

0

0

0

0

0

0

0

0

0

0

0

0

0

0

0

0

0

0

0

0

0

0

0

0

0

0

0

0

0

0

0

0

0

0

0

0

0

0

0

0

0

0

0

0

0

0

0

0 


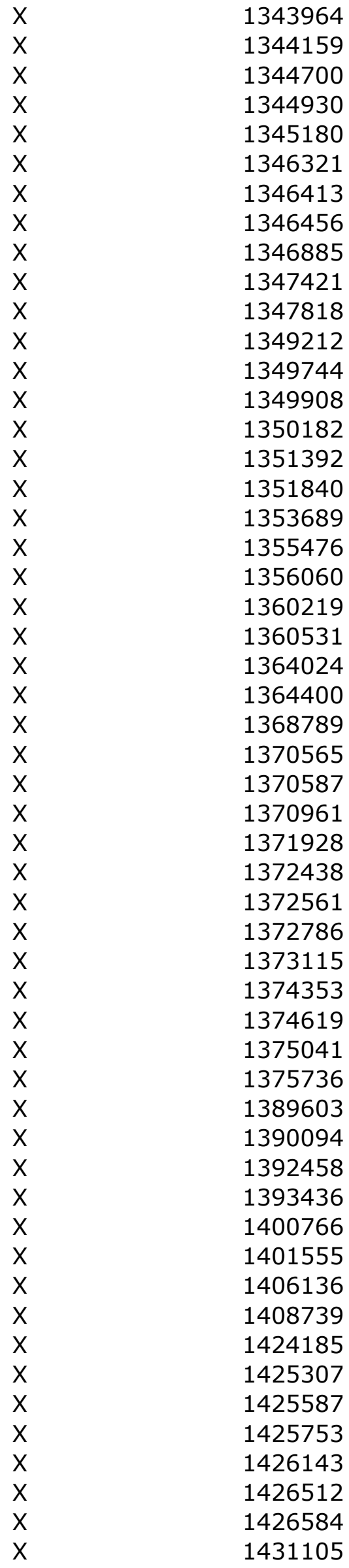

2
2
2
2
2
2
2
2
2
2
2
2
2
2
2
2
2
2
2
2
2
2
2
2
2
2
2
2
2
2
2
2
2
2
2
2
2
2
2
2
2
2
2
2
2
2
2
2
2
2
2
2
2
2
2
2
2
2
2
2
2

$\begin{array}{rr}8 & 1 \\ 9 & 1 \\ 22 & 2 \\ 5 & \\ 6 & 1 \\ 6 & 2 \\ 12 & 2 \\ 4 & 3 \\ 5 & 19 \\ 20 & 3\end{array}$

13

19

23

8

17

25

27

33

19

$18 \quad 29$

$8 \quad 28$

$8 \quad 15$

$6 \quad 14$

18

15

9

19

9

11

19

7

19

13

17

33

20

11

30

12

14

8

19

13

27

20

26

19

20

11

13

15

10

29

11

9

5

9

10

12

10

21

13

11

0

0

0

0

0

0

0

0

0

0

0

0

0

0

0

0

0

0

0

0

0

0

0

0

0

0

0

0

0

0

0

0

0

0

0

0

0

0

0

0

0

0 


\begin{tabular}{|c|c|}
\hline$x$ & 1431149 \\
\hline$x$ & 1431372 \\
\hline$x$ & 1431606 \\
\hline$x$ & 1431814 \\
\hline$x$ & 1431872 \\
\hline$x$ & 1431949 \\
\hline$x$ & 1432079 \\
\hline$x$ & 1444345 \\
\hline$x$ & 1455956 \\
\hline$x$ & 1458016 \\
\hline$x$ & 1479688 \\
\hline$x$ & 1486421 \\
\hline$x$ & 1488896 \\
\hline$x$ & 1499799 \\
\hline$x$ & 1504223 \\
\hline$x$ & 1508260 \\
\hline$x$ & 1511351 \\
\hline$x$ & 1521136 \\
\hline$x$ & 1521549 \\
\hline$x$ & 1529743 \\
\hline$x$ & 1529853 \\
\hline$x$ & 1531066 \\
\hline$x$ & 1533000 \\
\hline$x$ & 1533930 \\
\hline$x$ & 1537854 \\
\hline$x$ & 1543187 \\
\hline$x$ & 1544072 \\
\hline$x$ & 1545092 \\
\hline$x$ & 1546771 \\
\hline$x$ & 1547544 \\
\hline$x$ & 1547689 \\
\hline$x$ & 1548758 \\
\hline$x$ & 1549194 \\
\hline$x$ & 1549829 \\
\hline$x$ & 1549978 \\
\hline$x$ & 1550485 \\
\hline$x$ & 1550957 \\
\hline$x$ & 1551083 \\
\hline$x$ & 1551806 \\
\hline$x$ & 1552208 \\
\hline$x$ & 1552327 \\
\hline$x$ & 1554424 \\
\hline$x$ & 1558589 \\
\hline$x$ & 1559296 \\
\hline$x$ & 1559637 \\
\hline$x$ & 1559778 \\
\hline$x$ & 1560105 \\
\hline$x$ & 1560144 \\
\hline$x$ & 1561515 \\
\hline$x$ & 1561689 \\
\hline$x$ & 1563404 \\
\hline$x$ & 1563732 \\
\hline$X$ & 1563 \\
\hline
\end{tabular}

2

2

2

2

2

2

2

2

2

2

2

2

2

2

2

2

2

2

2

2

2

2

2

2

2

2

2

2

2

2

2

2

2

2

2

2

2

2

2

2

2

2

2

2

2

2

2

2

2

2

2

2

2
14

13

4

7

8

6

8

5

10

6

5

11

5

5

8

12

17

14

5

17

18

4

5

15

18

16

8

4

9

6

9

13

4

15

17

16

11

19

4

6

4

7

5

11

14

6

4

9

15

8

4

4

11
14

15

11

13

18

12

27

23

18

8

8

22

8

15

17

21

8

12

20

8

15

14

23

21

8

19

19

16

24

16

19

16

22

40

29

24

31

16

15

9

25

12

14

16

20

14

20

19

17

28

13

21

11
0

0

0

0

0

0

0

0

0

0

0

0

0

0

0

0

0

0

0

0

0

0

0

0

0

0

0

0

0

0

0

0

0

0

0

0

0

0

0

0

0

0

0

0

0

0

0

0

0

0 


\begin{tabular}{|c|c|}
\hline$x$ & 1564139 \\
\hline$x$ & 1568778 \\
\hline$x$ & 1569108 \\
\hline$x$ & 1571189 \\
\hline$x$ & 1571819 \\
\hline$x$ & 1572034 \\
\hline$x$ & 1572263 \\
\hline$x$ & 1572334 \\
\hline$x$ & 1572511 \\
\hline$x$ & 1572729 \\
\hline$x$ & 1576976 \\
\hline$x$ & 1580147 \\
\hline$x$ & 1581103 \\
\hline$x$ & 1585377 \\
\hline$x$ & 1586666 \\
\hline$x$ & 1592905 \\
\hline$x$ & 1593480 \\
\hline$x$ & 1598947 \\
\hline$x$ & 1599106 \\
\hline$x$ & 1599204 \\
\hline$x$ & 1601834 \\
\hline$x$ & 1601922 \\
\hline$x$ & 1602061 \\
\hline$x$ & 1602179 \\
\hline$x$ & 1602431 \\
\hline$x$ & 1602692 \\
\hline$x$ & 1602722 \\
\hline$x$ & 1602925 \\
\hline$x$ & 1604038 \\
\hline$x$ & 1604389 \\
\hline$x$ & 1604596 \\
\hline$x$ & 1604763 \\
\hline$x$ & 1604799 \\
\hline$x$ & 1605172 \\
\hline$x$ & 1606782 \\
\hline$x$ & 1607026 \\
\hline$x$ & 1607420 \\
\hline$x$ & 1607968 \\
\hline$x$ & 1609336 \\
\hline$x$ & 1612105 \\
\hline$x$ & 1616575 \\
\hline$x$ & 1616629 \\
\hline$x$ & 1617096 \\
\hline$x$ & 1618906 \\
\hline$x$ & 1619121 \\
\hline$x$ & 1629611 \\
\hline$x$ & 1630293 \\
\hline$x$ & 1630737 \\
\hline$x$ & 1640508 \\
\hline$x$ & 1640679 \\
\hline$x$ & 1642238 \\
\hline$x$ & 1643588 \\
\hline . & 164 \\
\hline
\end{tabular}

2

2

2

2

2

2

2

2

2

2

2

2

2

2

2

2

2

2

2

2

2

2

2

2

2

2

2

2

2

2

2

2

2

2

2

2

2

2

2

2

2

2

2

2

2

2

2

2

2

2

2

2

2
12

4

10

15

5

23

30

18

10

16

22

4

18

4

12

12

16

4

10

13

22

17

30

33

36

9

8

21

19

43

18

23

20

4

21

11

7

15

29

18

4

9

7

21

20

11

5

11

17

4

25

8
34

17

22

22

29

30

15

35

37

13

12

10

13

14

9

24

4

19

19

18

15

5

18

3

11

6

14

10

4

14

10

11

4

9

5

4

15

12

5

9

25

15

23

7

10

18

15

20

18

29

7

8

6
0

0

0

0

0

0

0

0

0

0

0

0

0

0

0

0

0

0

0

0

0

0

0

0

0

0

0

0

0

0

0

0

0

0

0

0

0

0

0

0

0

0

0

0

0

0

0

0 


\begin{tabular}{|c|c|}
\hline$x$ & 1644751 \\
\hline$x$ & 1646144 \\
\hline$X$ & 1646614 \\
\hline$x$ & 1647021 \\
\hline$x$ & 1647080 \\
\hline$x$ & 1647169 \\
\hline$x$ & 1647692 \\
\hline$x$ & 1647759 \\
\hline$x$ & 1647793 \\
\hline$x$ & 1647908 \\
\hline$x$ & 1647996 \\
\hline$x$ & 1648495 \\
\hline$x$ & 1648775 \\
\hline$x$ & 1649183 \\
\hline$X$ & 1649358 \\
\hline$x$ & 1649822 \\
\hline$x$ & 1649942 \\
\hline$x$ & 1650148 \\
\hline$x$ & 1650966 \\
\hline$x$ & 1651135 \\
\hline$x$ & 1652110 \\
\hline$x$ & 1652158 \\
\hline$x$ & 1654280 \\
\hline$x$ & 1655087 \\
\hline$x$ & 1656229 \\
\hline$x$ & 1656519 \\
\hline$x$ & 1656567 \\
\hline$x$ & 1664344 \\
\hline$x$ & 1673049 \\
\hline$x$ & 1701242 \\
\hline$x$ & 1711898 \\
\hline$x$ & 1714032 \\
\hline$x$ & 1714210 \\
\hline$x$ & 1714271 \\
\hline$x$ & 1714619 \\
\hline$x$ & 1715478 \\
\hline$x$ & 1715591 \\
\hline$x$ & 1715731 \\
\hline$x$ & 1716286 \\
\hline$x$ & 1716603 \\
\hline$x$ & 1716696 \\
\hline$x$ & 1716876 \\
\hline$x$ & 1717680 \\
\hline$x$ & 1717718 \\
\hline$x$ & 1718697 \\
\hline$x$ & 1718741 \\
\hline$x$ & 1721209 \\
\hline$x$ & 1724122 \\
\hline$x$ & 1724811 \\
\hline$x$ & 1724853 \\
\hline$x$ & 1727275 \\
\hline$x$ & 1728302 \\
\hline$x$ & 173463 \\
\hline
\end{tabular}

2

2

2

2

2

2

2

2

2

2

2

2

2

2

2

2

2

2

2

2

2

2

2

2

2

2

2

2

2

2

2

2

2

2

2

2

2

2

2

2

2

2

2

2

2

2

2

2

2

2

2

2

2
28

12

6

14

5

10

3

3

13

15

16

15

4

7
7

7

4
8

17

7

8

7

7

6

4

4

9

8

8

11

14

18

7

19

4

14

12

7

5

10

18

15

5

4

6

10

9

27

15

9

10

14

12

11
19

5

16

21

19

19

36

31

20

21

22

13

13

18

17

19

21

19

12

7

5

13
15

15

20

9

19

13

11

25

13

11

24

36

16

21

29

22

11

21

32

25

16

18

16

16

30

15

21

4

8

14

4

10
0

0

0

0

0

0

0

0

0

0

0

0

0

0

0

0

0

0

0

0

0

0

0

0

0

0

0

0

0

0

0

0

0

0

0

0

0

0

0

0

0

0

0

0

0

0

0

0

0

0 


\begin{tabular}{|c|c|c|c|c|c|}
\hline$x$ & 1737116 & 2 & 13 & 7 & 0 \\
\hline$x$ & 1740990 & 2 & 5 & 16 & 0 \\
\hline$x$ & 1744963 & 2 & 14 & 22 & 0 \\
\hline$x$ & 1748167 & 2 & 8 & 6 & 0 \\
\hline$x$ & 1751723 & 2 & 10 & 21 & 0 \\
\hline$x$ & 1766568 & 2 & 5 & 24 & 0 \\
\hline$x$ & 1766605 & 2 & 5 & 17 & 0 \\
\hline$x$ & 1766832 & 2 & 14 & 20 & 0 \\
\hline$x$ & 1767952 & 2 & 5 & 11 & 0 \\
\hline$x$ & 1768086 & 2 & 7 & 20 & 0 \\
\hline$x$ & 1768897 & 2 & 10 & 21 & 0 \\
\hline$x$ & 1769515 & 2 & 8 & 7 & 0 \\
\hline$x$ & 1769936 & 2 & 4 & 15 & 0 \\
\hline$x$ & 1770935 & 2 & 11 & 9 & 0 \\
\hline$x$ & 1773140 & 2 & 7 & 7 & 0 \\
\hline$x$ & 1773387 & 2 & 22 & 24 & 0 \\
\hline$x$ & 1773925 & 2 & 9 & 16 & 0 \\
\hline$x$ & 1779603 & 2 & 9 & 35 & 0 \\
\hline$x$ & 1783154 & 2 & 5 & 18 & 0 \\
\hline$x$ & 1785563 & 2 & 5 & 8 & 0 \\
\hline$x$ & 1789339 & 2 & 13 & 16 & 0 \\
\hline$x$ & 1792778 & 2 & 17 & 22 & 0 \\
\hline$x$ & 1794940 & 2 & 16 & 23 & 0 \\
\hline$x$ & 1795189 & 2 & 4 & 29 & 0 \\
\hline$x$ & 1795872 & 2 & 4 & 20 & 0 \\
\hline$x$ & 1796940 & 2 & 16 & 22 & 0 \\
\hline$x$ & 1802196 & 2 & 11 & 12 & 0 \\
\hline$x$ & 1813236 & 2 & 4 & 16 & 0 \\
\hline$x$ & 1814777 & 2 & 8 & 16 & 0 \\
\hline$x$ & 1817388 & 2 & 13 & 24 & 0 \\
\hline$x$ & 1817624 & 2 & 5 & 37 & 0 \\
\hline$x$ & 1818569 & 2 & 24 & 10 & 0 \\
\hline$x$ & 1820377 & 2 & 22 & 10 & 0 \\
\hline$x$ & 1823012 & 2 & 6 & 21 & 0 \\
\hline$x$ & 1838634 & 2 & 17 & 6 & 0 \\
\hline$x$ & 1842099 & 2 & 22 & 14 & 0 \\
\hline$x$ & 1845445 & 2 & 8 & 12 & 0 \\
\hline$x$ & 1847106 & 2 & 14 & 7 & 0 \\
\hline$x$ & 1859293 & 2 & 7 & 14 & 0 \\
\hline$x$ & 1861329 & 2 & 6 & 16 & 0 \\
\hline$x$ & 1864241 & 2 & 14 & 6 & 0 \\
\hline$x$ & 1866400 & 2 & 6 & 7 & 0 \\
\hline$x$ & 1868231 & 2 & 8 & 26 & 0 \\
\hline$x$ & 1869484 & 2 & 15 & 5 & 0 \\
\hline$x$ & 1874130 & 2 & 13 & 10 & 0 \\
\hline$x$ & 1874204 & 2 & 16 & 11 & 0 \\
\hline$x$ & 1878090 & 2 & 24 & 12 & 0 \\
\hline$x$ & 1879594 & 2 & 11 & 6 & 0 \\
\hline$x$ & 1879897 & 2 & 17 & 7 & 0 \\
\hline$x$ & 1881439 & 2 & 6 & 6 & 0 \\
\hline$x$ & 1890050 & 2 & 16 & 7 & 0 \\
\hline$x$ & 1891119 & 2 & 13 & 8 & 0 \\
\hline$x$ & 1892423 & 2 & 13 & 4 & 0 \\
\hline
\end{tabular}




\begin{tabular}{|c|c|}
\hline$x$ & 1892668 \\
\hline$x$ & 1893140 \\
\hline$x$ & 1893590 \\
\hline$x$ & 1897606 \\
\hline$x$ & 1898524 \\
\hline$x$ & 1899304 \\
\hline$x$ & 1901006 \\
\hline$x$ & 1902204 \\
\hline$x$ & 1904151 \\
\hline$x$ & 1904337 \\
\hline$x$ & 1906087 \\
\hline$x$ & 1907556 \\
\hline$x$ & 1907617 \\
\hline$x$ & 1908658 \\
\hline$x$ & 1912567 \\
\hline$x$ & 1913190 \\
\hline$x$ & 1914463 \\
\hline$x$ & 1914517 \\
\hline$x$ & 1925704 \\
\hline$x$ & 1928000 \\
\hline$x$ & 1929952 \\
\hline$x$ & 1935172 \\
\hline$x$ & 1935561 \\
\hline$x$ & 1936455 \\
\hline$x$ & 1936583 \\
\hline$x$ & 1944947 \\
\hline$x$ & 1946221 \\
\hline$x$ & 1950139 \\
\hline$x$ & 1950147 \\
\hline$x$ & 1950522 \\
\hline$x$ & 1954261 \\
\hline$x$ & 1954901 \\
\hline$x$ & 1955038 \\
\hline$x$ & 1955500 \\
\hline$x$ & 1958445 \\
\hline$x$ & 1958482 \\
\hline$x$ & 1963558 \\
\hline$x$ & 1965071 \\
\hline$x$ & 1965415 \\
\hline$x$ & 1965894 \\
\hline$x$ & 1966057 \\
\hline$x$ & 1966212 \\
\hline$x$ & 1966345 \\
\hline$x$ & 1966439 \\
\hline$x$ & 1967335 \\
\hline$X$ & 1967685 \\
\hline$x$ & 1968192 \\
\hline$x$ & 1968446 \\
\hline$x$ & 1969139 \\
\hline$x$ & 1969152 \\
\hline$X$ & 1970678 \\
\hline$X$ & 1970788 \\
\hline$x$ & 1971 \\
\hline
\end{tabular}

2

2

2

2

2

2

2

2

2

2

2

2

2

2

2

2

2

2

2

2

2

2

2

2

2

2

2

2

2

2

2

2

2

2

2

2

2

2

2

2

2

2

2

2

2

2

2

2

2

2

2

2

2
13

9

10

30

4

19

26

19

8

5

6

6

7

14

21

11

24

18

11

7

17

24

22

8

5

14

11

12

11

14

18

5

5

27

30

34

19

22

20

29

17

20

12

22

15

23

19

16

19

28

21

22

23
23

5
4

18

22

14

9

15

29

9

22

25

6

12

30

20

29

20

27

14

7

10

14

5

10

5

10

23

24

24

8

10

9

15

14

13

4

7

11

14

8

26

6

7

6

13

6

4

6

9

12

13

7
0

0

0

0

0

0

0

0

0

0

0

0

0

0

0

0

0

0

0

0

0

0

0

0

0

0

0

0

0

0

0

0

0

0

0

0

0

0

0

0

0

0

0

0

0

0

0

0

0

0 


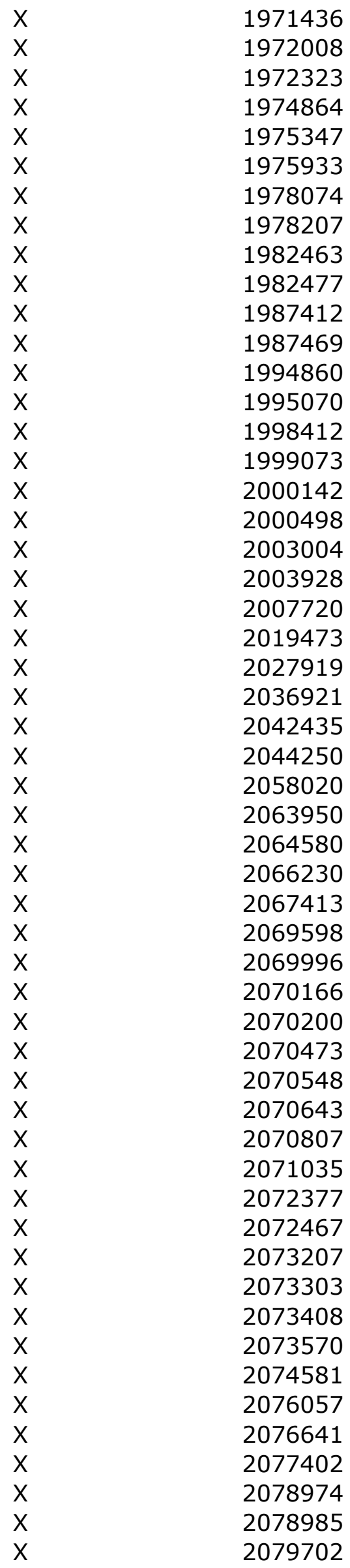

2

2

2

2

2

2

2

2

2

2

2

2

2

2

2

2

2

2

2

2

2

2

2

2

2

2

2

2

2

2

2

2

2

2

2

2

2

2

2

2

2

2

2

2

2

2

2

2

2

2

2

2

2
15

9

24

14

10

16

33

29

15

13

24

8

7

12

11

14

30

11

9

4

7

10

6

10

11

4

5

8

11

27

14

22

17

14

15

32

11

15

8

4

13

6

12

16

26

14

16

7

7

7

6

19

5
12

14

18

5

23

23

12

10

6

16

17

5

13

15

5

8

15

6

14

9

13

4

17

7

15

10

7

21

5

6

21

10

19

4

7

14

4

5

19

15

30

24

22

32

24

13

28

21

19

25

10

10

19
0

0

0

0

0

0

0

0

0

0

0

0

0

0

0

0

0

0

0

0

0

0

0

0

0

0

0

0

0

0

0

0

0

0

0

0

0

0

0

0

0

0

0

0

0

0

0

0

0

0 


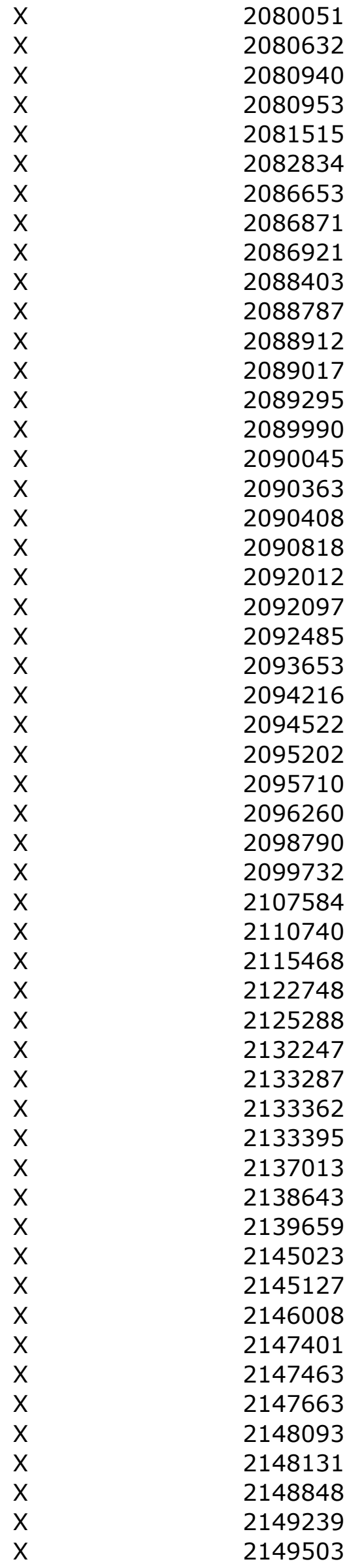

$\begin{array}{lr}2 & 7 \\ 2 & 6 \\ 2 & 21 \\ 2 & 26 \\ 2 & 5 \\ 2 & 24 \\ 2 & 4 \\ 2 & 9 \\ 2 & 7\end{array}$

14

15

17

12

12

24

9

10

19

24

30

13

16

4

19

17

13

15

29

9

18

17

21

11

34

17

15

13

19

29

32

21

13

33

12

15

12

16

23

15

17

14

13

24

26

4

8

21

8

12

11

4

4

6

5

9

6

29

28

33

34

12

16

13

27

19

9

14

5

12

7

4

20

13

26

$11 \quad 13 \quad 0$

17

$\begin{array}{lll}15 & 28 & 0\end{array}$

$21 \quad 16 \quad 0$

32

$\begin{array}{lll}4 & 20 & 0\end{array}$

19

26

26

26
6

0

0

0

0

0

0

0

0

0

0

0

0

0

0

0

0

0

0

0

0

0

0

0

0

0

0

0

0

0

0

0

0

0

0

0

0

0

0

11

12

8
8

0

0 


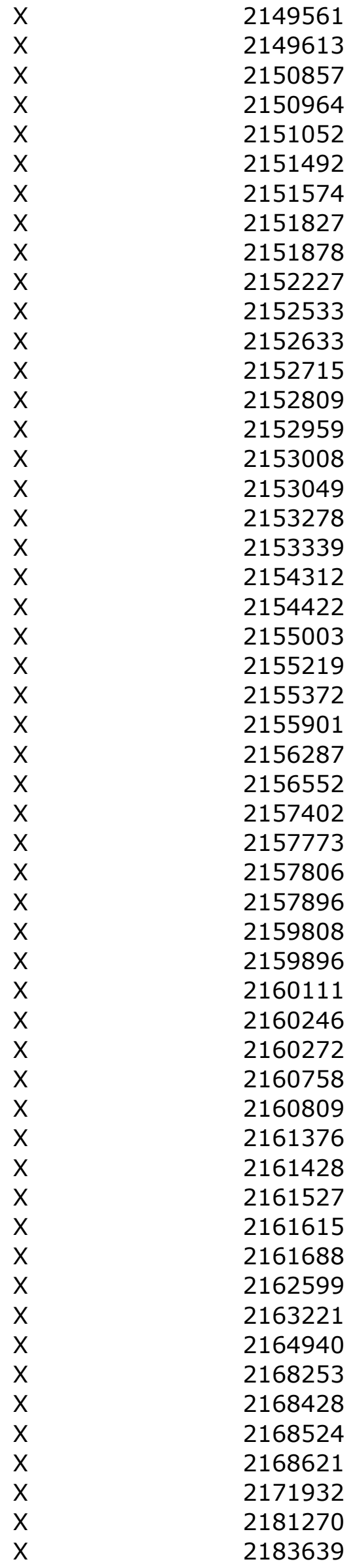

2

2

2

2

2

2

2

2

2

2

2

2

2

2

2

2

2

2

2

2

2

2

2

2

2

2

2

2

2

2

2

2

2

2

2

2

2

2

2

2

2

2

2

2

2

2

2

2

2

2

2

2

2
22

13

14

6

12

7

7

9

4

5

9

4

4

11

24

21

10

11

20

13

12

21

20

11

19

5

5

5

13

12

23

9

15

12

14

10

6

9

9

6

6

19

5

17

6

14

11

10

5

4

10

9

13
15

4

40

12

13

10

26

16

25

15

19

16

13

17

9

6

10

6

10

12

18

4

11

7

5

13

15

7

7

9

15

21

27

17

34

32

16

19

9

23

8

8

17

27

9

5

21

17

15

24

31

25

12
0

0

0

0

0

0

0

0

0

0

0

0

0

0

0

0

0

0

0

0

0

0

0

0

0

0

0

0

0

0

0

0

0

0

0

0

0

0

0

0

0

0

0

0

0

0

0

0

0

0 


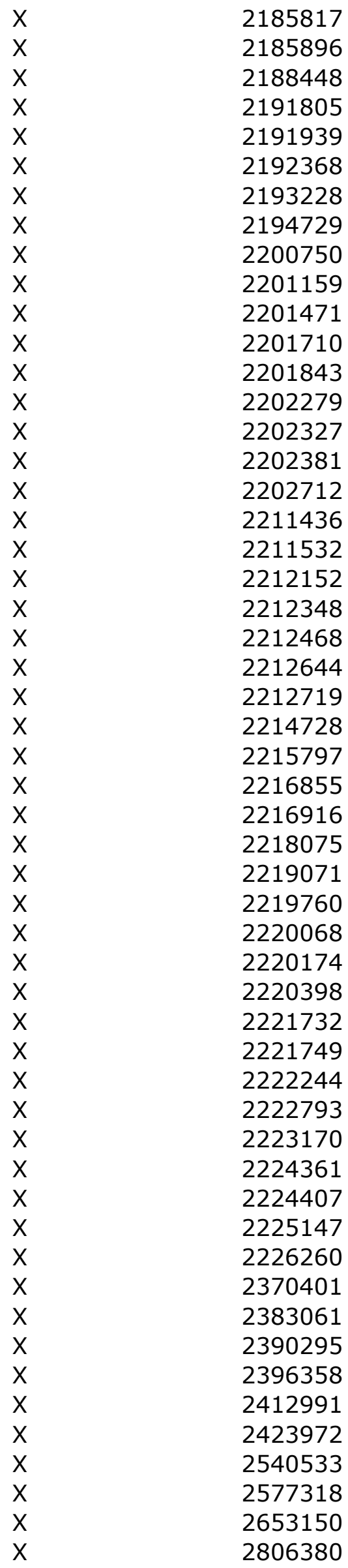

\begin{tabular}{|c|c|c|c|}
\hline 2 & 10 & 4 & 0 \\
\hline 2 & 7 & 11 & 0 \\
\hline 2 & 24 & 8 & 0 \\
\hline 2 & 15 & 15 & 0 \\
\hline 2 & 10 & 11 & 0 \\
\hline 2 & 22 & 28 & 0 \\
\hline 2 & 7 & 29 & 0 \\
\hline 2 & 10 & 25 & 0 \\
\hline 2 & 13 & 16 & 0 \\
\hline 2 & 13 & 7 & 0 \\
\hline 2 & 15 & 4 & 0 \\
\hline 2 & 38 & 13 & 0 \\
\hline 2 & 14 & 7 & 0 \\
\hline 2 & 16 & 4 & 0 \\
\hline 2 & 5 & 6 & 0 \\
\hline 2 & 27 & 11 & 0 \\
\hline 2 & 8 & 5 & 0 \\
\hline 2 & 13 & 4 & 0 \\
\hline 2 & 8 & 10 & 0 \\
\hline 2 & 22 & 21 & 0 \\
\hline 2 & 26 & 7 & 0 \\
\hline 2 & 5 & 34 & 0 \\
\hline 2 & 4 & 23 & 0 \\
\hline 2 & 22 & 17 & 0 \\
\hline 2 & 15 & 4 & 0 \\
\hline 2 & 21 & 4 & 0 \\
\hline 2 & 7 & 20 & 0 \\
\hline 2 & 6 & 21 & 0 \\
\hline 2 & 14 & 20 & 0 \\
\hline 2 & 20 & 4 & 0 \\
\hline 2 & 20 & 4 & 0 \\
\hline 2 & 10 & 9 & 0 \\
\hline 2 & 9 & 7 & 0 \\
\hline 2 & 8 & 11 & 0 \\
\hline 2 & 20 & 16 & 0 \\
\hline 2 & 14 & 18 & 0 \\
\hline 2 & 4 & 24 & 0 \\
\hline 2 & 15 & 23 & 0 \\
\hline 2 & 19 & 8 & 0 \\
\hline 2 & 10 & 32 & 0 \\
\hline 2 & 7 & 24 & 0 \\
\hline 2 & 19 & 10 & 0 \\
\hline 2 & 5 & 10 & 0 \\
\hline 2 & 5 & 5 & 0 \\
\hline 2 & 16 & 4 & 0 \\
\hline 2 & 6 & 8 & 0 \\
\hline 2 & 7 & 8 & 0 \\
\hline 2 & 5 & 5 & 0 \\
\hline 2 & 11 & 4 & 0 \\
\hline 2 & 10 & 4 & 0 \\
\hline 2 & 5 & 6 & 0 \\
\hline 2 & 9 & 4 & 0 \\
\hline 2 & 5 & 5 & 0 \\
\hline
\end{tabular}




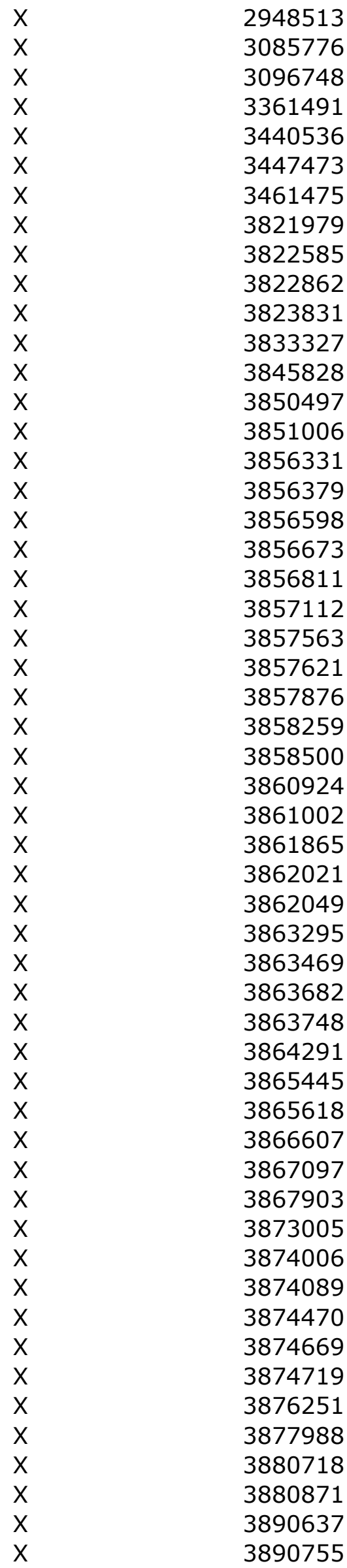

\begin{tabular}{|c|c|c|}
\hline 2 & 6 & 9 \\
\hline 2 & 6 & 6 \\
\hline 2 & 6 & 5 \\
\hline 2 & 6 & 6 \\
\hline 2 & 4 & 12 \\
\hline 2 & 5 & 5 \\
\hline 2 & 7 & 5 \\
\hline 2 & 4 & 18 \\
\hline 2 & 10 & 10 \\
\hline 2 & 8 & 6 \\
\hline 2 & 4 & 11 \\
\hline 2 & 10 & 5 \\
\hline 2 & 16 & 8 \\
\hline 2 & 9 & 6 \\
\hline 2 & 17 & 7 \\
\hline 2 & 23 & 6 \\
\hline 2 & 22 & 8 \\
\hline 2 & 26 & 4 \\
\hline 2 & 21 & 5 \\
\hline 2 & 7 & 7 \\
\hline 2 & 14 & 7 \\
\hline 2 & 21 & 9 \\
\hline 2 & 25 & 20 \\
\hline 2 & 17 & 9 \\
\hline 2 & 17 & 10 \\
\hline 2 & 13 & 8 \\
\hline 2 & 17 & 7 \\
\hline 2 & 14 & 26 \\
\hline 2 & 14 & 5 \\
\hline 2 & 4 & 20 \\
\hline 2 & 12 & 19 \\
\hline 2 & 17 & 8 \\
\hline 2 & 19 & 5 \\
\hline 2 & 14 & 22 \\
\hline 2 & 17 & 5 \\
\hline 2 & 10 & 4 \\
\hline 2 & 27 & 10 \\
\hline 2 & 7 & 12 \\
\hline 2 & 6 & 15 \\
\hline 2 & 5 & 15 \\
\hline 2 & 4 & 17 \\
\hline 2 & 10 & 17 \\
\hline 2 & 5 & 18 \\
\hline 2 & 15 & 23 \\
\hline 2 & 10 & 14 \\
\hline 2 & 4 & 22 \\
\hline 2 & 6 & 17 \\
\hline 2 & 18 & 4 \\
\hline 2 & 11 & 9 \\
\hline 2 & 20 & 30 \\
\hline 2 & 7 & 22 \\
\hline 2 & 8 & 13 \\
\hline 2 & 8 & 10 \\
\hline
\end{tabular}




$\begin{array}{ll}X & 3891142 \\ X & 3891593 \\ X & 3891955 \\ X & 3894816 \\ X & 3894877 \\ X & 3894992 \\ X & 3895591 \\ X & 3895838 \\ X & 3896310 \\ X & 3896933 \\ X & 3897679 \\ X & 3897812 \\ X & 3898476 \\ X & 3904584 \\ X & 3907049 \\ X & 3909387 \\ X & 3913619 \\ X & 3915165 \\ X & 3915561 \\ X & 3917444 \\ X & 3922259 \\ X & 3922668 \\ X & 3922847 \\ X & 3926664 \\ X & 3928163 \\ X & 3929193 \\ X & 3929296 \\ X & 3930491 \\ X & 3930823 \\ X & 3934182 \\ X & 3936895 \\ X & 3937507 \\ X & 3937863 \\ X & 3939230 \\ X & 3940134 \\ X & 3943048 \\ X & 3945087 \\ X & 3947415 \\ X & 3948585 \\ X & 3949334 \\ X & 3950574 \\ X & 3950633 \\ X & 3950648 \\ X & 3953588 \\ X & 3955308 \\ X & \\ X & 3956632 \\ X & 416867 \\ X & \end{array}$

2

2

2

2

2

2

2

2

2

2

2

2

2

2

2

2

2

2

2

2

2

2

2

2

2

2

2

2

2

2

2

2

2

2

2

2

2

2

2

2

2

2

2

2

2

2

2

2

2

2

2

2

2

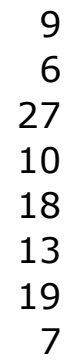

12

10

8

21

17

4

7

26

6

9
17

17

7

7

12

7

26

16

18

4

13

10

24

12

13

4

13

10

18

10

6

12

23

23

15

6

16

16

13

4

12

10

5

10

9
7

8

4

4

8

11

10

6

35

5

20

4

19

21

7

24

4

4

23

20

22

13

14

10

7

10

14

15

12

9

6

9

22

22

4

20

19

4

14

9

13

12

10

7

6

15

7

5

5

7

5

5
0

0

0

0

0

0

0

0

0

0

0

0

0

0

0

0

0

0

0

0

0

0

0

0

0

0

0

0

0

0

0

0

0

0

0

0

0

0

0

0

0

0

0

0

0

0

0

0

0

0 


\begin{tabular}{|c|c|}
\hline$x$ & 4315317 \\
\hline$x$ & 4324268 \\
\hline$x$ & 4343964 \\
\hline$x$ & 4344267 \\
\hline$x$ & 4350318 \\
\hline$x$ & 4352314 \\
\hline$x$ & 4358444 \\
\hline$x$ & 4359676 \\
\hline$x$ & 4362182 \\
\hline$x$ & 4362631 \\
\hline$x$ & 4362683 \\
\hline$x$ & 4364063 \\
\hline$x$ & 4364090 \\
\hline$X$ & 4366096 \\
\hline$x$ & 4366334 \\
\hline$x$ & 4366458 \\
\hline$x$ & 4372537 \\
\hline$x$ & 4376227 \\
\hline$x$ & 4379875 \\
\hline$x$ & 4380980 \\
\hline$x$ & 4387452 \\
\hline$x$ & 4387759 \\
\hline$x$ & 4391602 \\
\hline$x$ & 4391858 \\
\hline$x$ & 4393058 \\
\hline$x$ & 4394067 \\
\hline$x$ & 4397785 \\
\hline$x$ & 4398192 \\
\hline$x$ & 4399125 \\
\hline$x$ & 4401421 \\
\hline$x$ & 4411912 \\
\hline$x$ & 4414228 \\
\hline$x$ & 4416638 \\
\hline$x$ & 4418108 \\
\hline$x$ & 4418557 \\
\hline$x$ & 4419765 \\
\hline$x$ & 4421330 \\
\hline$x$ & 4423626 \\
\hline$x$ & 4424162 \\
\hline$x$ & 4424560 \\
\hline$x$ & 4424762 \\
\hline$x$ & 4425357 \\
\hline$x$ & 4425385 \\
\hline$x$ & 4425553 \\
\hline$x$ & 4426029 \\
\hline$x$ & 4426053 \\
\hline$x$ & 4427040 \\
\hline$x$ & 4429167 \\
\hline$x$ & 4430198 \\
\hline$x$ & 4430513 \\
\hline$x$ & 4431334 \\
\hline$x$ & 4431388 \\
\hline$x$ & 4432108 \\
\hline
\end{tabular}

\begin{tabular}{|c|c|c|}
\hline 8 & 5 & 0 \\
\hline 11 & 6 & 0 \\
\hline 13 & 6 & 0 \\
\hline 16 & 6 & 0 \\
\hline 12 & 4 & 0 \\
\hline 8 & 15 & 0 \\
\hline 14 & 18 & 0 \\
\hline 15 & 9 & 0 \\
\hline 28 & 13 & 0 \\
\hline 11 & 4 & 0 \\
\hline 11 & 5 & 0 \\
\hline 10 & 17 & 0 \\
\hline 5 & 12 & 0 \\
\hline 20 & 21 & 0 \\
\hline 19 & 14 & 0 \\
\hline 23 & 13 & 0 \\
\hline 8 & 18 & 0 \\
\hline 5 & 8 & 0 \\
\hline 6 & 13 & 0 \\
\hline 6 & 18 & 0 \\
\hline 9 & 6 & 0 \\
\hline 4 & 11 & 0 \\
\hline 6 & 7 & 0 \\
\hline 12 & 7 & 0 \\
\hline 13 & 9 & 0 \\
\hline 19 & 5 & 0 \\
\hline 21 & 10 & 0 \\
\hline 4 & 23 & 0 \\
\hline 14 & 27 & 0 \\
\hline 6 & 12 & 0 \\
\hline 10 & 21 & 0 \\
\hline 10 & 24 & 0 \\
\hline 19 & 6 & 0 \\
\hline 7 & 10 & 0 \\
\hline 6 & 10 & 0 \\
\hline 13 & 9 & 0 \\
\hline 7 & 8 & 0 \\
\hline 15 & 6 & 0 \\
\hline 8 & 9 & 0 \\
\hline 7 & 6 & 0 \\
\hline 7 & 9 & 0 \\
\hline 15 & 15 & 0 \\
\hline 24 & 15 & 0 \\
\hline 8 & 13 & 0 \\
\hline 5 & 7 & 0 \\
\hline 4 & 10 & 0 \\
\hline 7 & 22 & 0 \\
\hline 16 & 5 & 0 \\
\hline 12 & 12 & 0 \\
\hline 4 & 12 & 0 \\
\hline 27 & 9 & 0 \\
\hline 11 & 6 & 0 \\
\hline 5 & 10 & U \\
\hline
\end{tabular}




\begin{tabular}{|c|c|}
\hline$x$ & 4433003 \\
\hline$x$ & 4433253 \\
\hline$x$ & 4433403 \\
\hline$x$ & 4434162 \\
\hline$x$ & 4434354 \\
\hline$x$ & 4434502 \\
\hline$x$ & 4435296 \\
\hline$x$ & 4441463 \\
\hline$x$ & 4443812 \\
\hline$x$ & 4447851 \\
\hline X & 4455420 \\
\hline$x$ & 4461691 \\
\hline$x$ & 4461711 \\
\hline$x$ & 4462433 \\
\hline$x$ & 4462958 \\
\hline$x$ & 4463117 \\
\hline$x$ & 4465678 \\
\hline$x$ & 4467499 \\
\hline$x$ & 4469211 \\
\hline$x$ & 4469275 \\
\hline$x$ & 4469394 \\
\hline$x$ & 4469835 \\
\hline$x$ & 4472481 \\
\hline$x$ & 4476158 \\
\hline$x$ & 4481046 \\
\hline$x$ & 4481490 \\
\hline$x$ & 4485072 \\
\hline$x$ & 4486618 \\
\hline$x$ & 4487149 \\
\hline$x$ & 4487355 \\
\hline$x$ & 4488148 \\
\hline$x$ & 4488609 \\
\hline$x$ & 4490045 \\
\hline$x$ & 4490520 \\
\hline$x$ & 4491492 \\
\hline$x$ & 4491979 \\
\hline$x$ & 4492974 \\
\hline$x$ & 4498309 \\
\hline$x$ & 4498725 \\
\hline$x$ & 4500109 \\
\hline$x$ & 4510393 \\
\hline$x$ & 4510459 \\
\hline$x$ & 4514854 \\
\hline$x$ & 4516138 \\
\hline$x$ & 4519489 \\
\hline$x$ & 4520016 \\
\hline$x$ & 4520119 \\
\hline$x$ & 4520820 \\
\hline$x$ & 4522861 \\
\hline$x$ & 4526638 \\
\hline$x$ & 4530572 \\
\hline$X$ & 4532126 \\
\hline$x$ & 4532149 \\
\hline
\end{tabular}

$2 \quad 15$

$2 \quad 10$

25

2

2

2

2

2

2

2

2

2

2

2

220

$2 \quad 15$

$2 \quad 12$

26

$2 \quad 17$

$2 \quad 10$

24

27

25

$2 \quad 18$

$2 \quad 19$

$2 \quad 11$

$2 \quad 15$

$2 \quad 10$

2

2

2

2

2

2

2

2

2

2

2

2

2

2

2

2

2

2

2

2

2

2

2

2
9

4

23

10

11

22

29

14

18

7

18

5

4

9

7

4

4

16

29

18

20

37

17

16

7

11

28

24

18

12

11

21

16

26

25

13

21

14

7

24

14

13

4

30

6

29

4

18

19

31

14

26

21
0

0

0

0

0

0

0

0

0

0

0

0

0

0

0

0

0

0

0

0

0

0

0

0

0

0

0

0

0

0

0

0

0

0

0

0

0

0

0

0

0

0

0

0

0

0

0

0

0

0

0 


\begin{tabular}{|c|c|}
\hline$x$ & 4532835 \\
\hline$x$ & 4533152 \\
\hline$x$ & 4536891 \\
\hline$x$ & 4537361 \\
\hline$x$ & 4538953 \\
\hline$X$ & 4539927 \\
\hline$x$ & 4541941 \\
\hline$X$ & 4543569 \\
\hline$x$ & 4547002 \\
\hline$X$ & 4548151 \\
\hline$x$ & 4550761 \\
\hline$X$ & 4551338 \\
\hline$x$ & 4553200 \\
\hline$X$ & 4554273 \\
\hline$x$ & 4555535 \\
\hline$x$ & 4555598 \\
\hline$X$ & 4556249 \\
\hline$X$ & 4557376 \\
\hline$x$ & 4562164 \\
\hline$x$ & 4563212 \\
\hline$x$ & 4563392 \\
\hline$x$ & 4564518 \\
\hline$X$ & 4566057 \\
\hline$X$ & 4566123 \\
\hline$x$ & 4568625 \\
\hline$x$ & 4570370 \\
\hline$x$ & 4571328 \\
\hline$X$ & 4571596 \\
\hline$x$ & 4573268 \\
\hline$X$ & 4573444 \\
\hline$X$ & 4573617 \\
\hline$X$ & 4574151 \\
\hline$x$ & 4574815 \\
\hline$x$ & 4575007 \\
\hline$x$ & 4575447 \\
\hline$x$ & 4575871 \\
\hline$x$ & 4576081 \\
\hline$X$ & 4586018 \\
\hline$x$ & 4586351 \\
\hline$x$ & 4590617 \\
\hline$x$ & 4593287 \\
\hline$X$ & 4598184 \\
\hline$x$ & 4598343 \\
\hline$x$ & 4599333 \\
\hline$x$ & 4599916 \\
\hline$x$ & 4603851 \\
\hline$X$ & 4606877 \\
\hline$x$ & 4609181 \\
\hline$x$ & 4611412 \\
\hline$X$ & 4611460 \\
\hline$x$ & 4611954 \\
\hline$x$ & 4612596 \\
\hline$x$ & 4613016 \\
\hline
\end{tabular}

\section{2}

2

2

2

2

2

2

2

2

2

2

2

2

2

2

2

2

2

2

2

2

2

2

2

2

2

2

2

2

2

2

2

2

2

2

2

2

2

2

2

2

2

2

2

2

2

2

2

2

2

2

2

2

$\begin{array}{rr}8 & 1 \\ 16 & 1 \\ 10 & \\ 7 & \\ 7 & \\ 9 & \\ 15 & \\ 12 & \end{array}$

33

10

7

6

14

7

15

23

11

8

10

6

8

7

13

9

8

5

6

13

5

10

26

15

16

8

10

4

10

22

15

8

13

11

16

9

12

6

5

7

17

8

12

12

22
16

12

6
7

8

13

4
4

17

18

26

21

16

5

5

11

24

12

25

28

5

13

13

27

13

25

18

8

22

4

21

8

14

8

14

22

22

4

4

20

19

21

13

11

4

21

22

13

6

13

9

16

17
0

0

0

0

0

0

0

0

0

0

0

0

0

0

0

0

0

0

0

0

0

0

0

0

0

0

0

0

0

0

0

0

0

0

0

0

0

0

0

0

0

0

0

0

0

0

0

0

0 


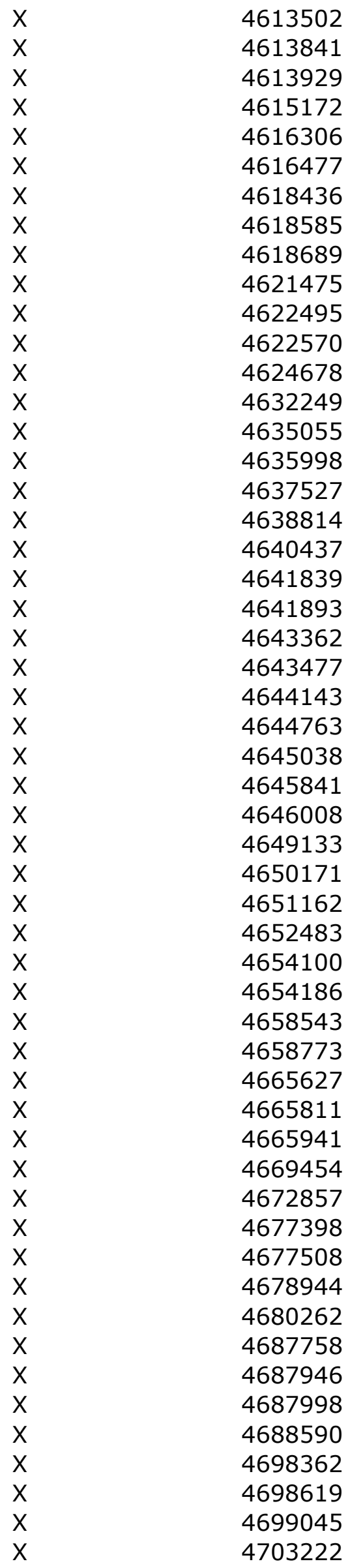

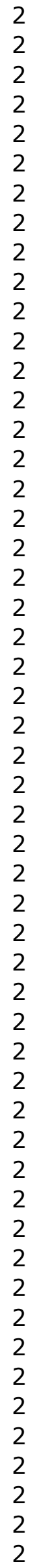

\begin{tabular}{|c|c|c|}
\hline 9 & 8 & 0 \\
\hline 18 & 15 & 0 \\
\hline 8 & 6 & 0 \\
\hline 9 & 19 & 0 \\
\hline 8 & 11 & 0 \\
\hline 14 & 10 & 0 \\
\hline 13 & 31 & 0 \\
\hline 10 & 11 & 0 \\
\hline 8 & 15 & 0 \\
\hline 11 & 26 & 0 \\
\hline 4 & 13 & 0 \\
\hline 5 & 19 & 0 \\
\hline 8 & 20 & 0 \\
\hline 13 & 9 & 0 \\
\hline 21 & 6 & 0 \\
\hline 10 & 7 & 0 \\
\hline 4 & 25 & 0 \\
\hline 17 & 5 & 0 \\
\hline 9 & 6 & 0 \\
\hline 11 & 5 & 0 \\
\hline 11 & 7 & \\
\hline 12 & 5 & 0 \\
\hline 7 & 13 & 0 \\
\hline 16 & 6 & 0 \\
\hline 5 & 18 & 0 \\
\hline 9 & 7 & 0 \\
\hline 7 & 6 & 0 \\
\hline 14 & 5 & 0 \\
\hline 9 & 10 & 0 \\
\hline 11 & 5 & 0 \\
\hline 5 & 12 & 0 \\
\hline 4 & 19 & 0 \\
\hline 8 & 19 & 0 \\
\hline 7 & 23 & 0 \\
\hline 5 & 7 & 0 \\
\hline 12 & 22 & 0 \\
\hline 24 & 7 & 0 \\
\hline 7 & 5 & 0 \\
\hline 16 & 7 & 0 \\
\hline 22 & 12 & 0 \\
\hline 6 & 7 & 0 \\
\hline 5 & 28 & 0 \\
\hline 9 & 16 & 0 \\
\hline 15 & 5 & 0 \\
\hline 12 & 13 & 0 \\
\hline 4 & 13 & 0 \\
\hline 7 & 15 & 0 \\
\hline 5 & 19 & 0 \\
\hline 4 & 10 & 0 \\
\hline 9 & 12 & 0 \\
\hline 7 & 25 & 0 \\
\hline 6 & 12 & 0 \\
\hline 4 & 16 & $U$ \\
\hline
\end{tabular}




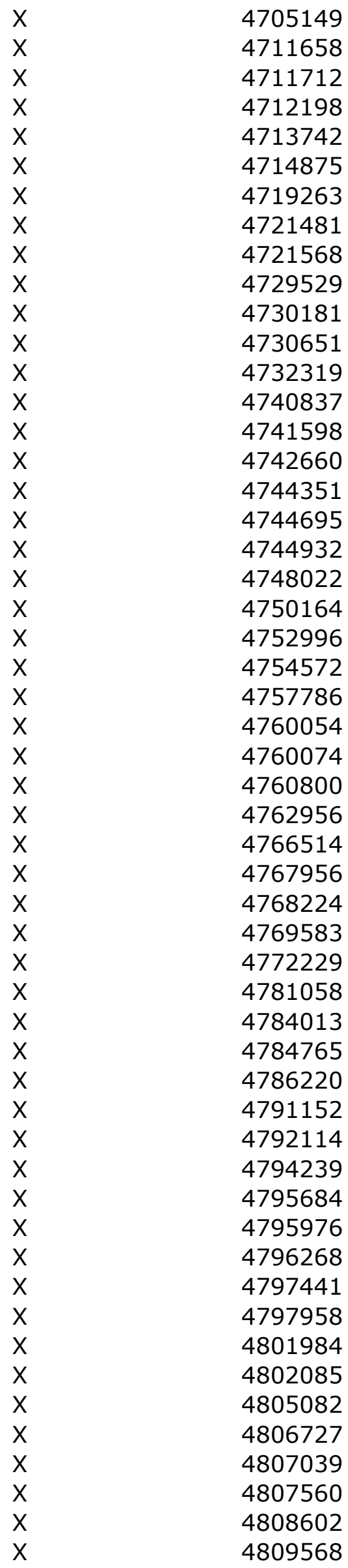

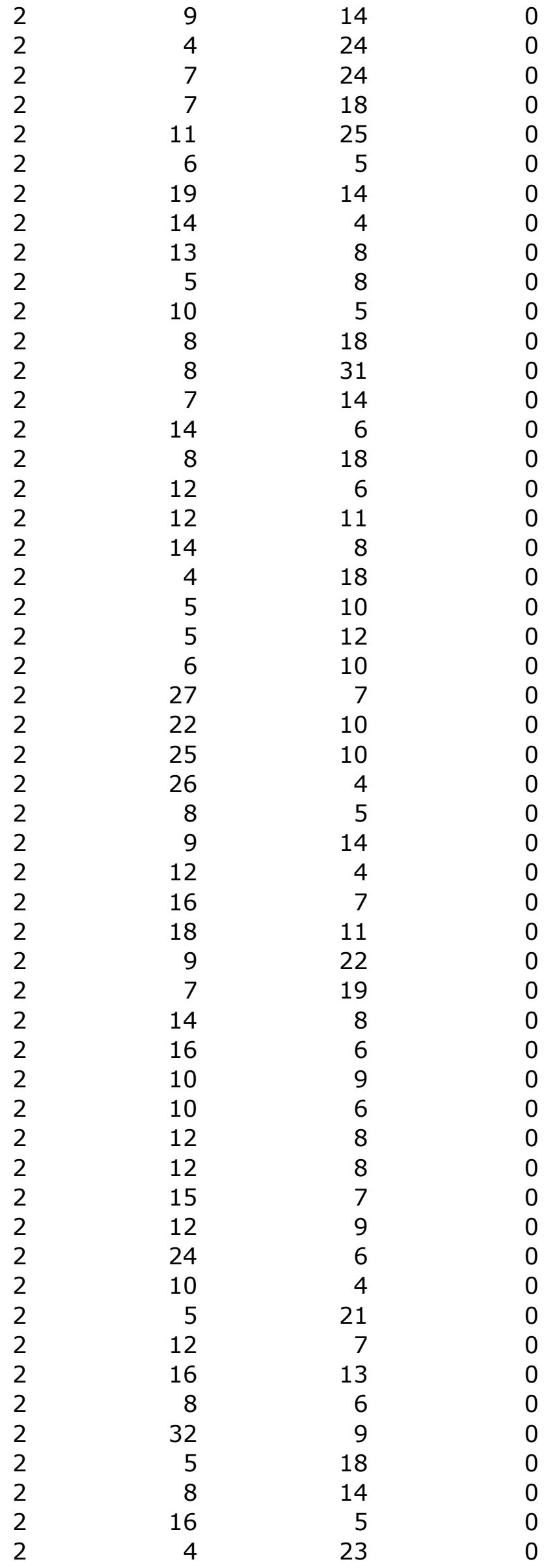




\begin{tabular}{|c|c|}
\hline$x$ & 4810340 \\
\hline$x$ & 4811711 \\
\hline$x$ & 4828807 \\
\hline$x$ & 4832397 \\
\hline $\mathrm{X}$ & 4835487 \\
\hline$x$ & 4836041 \\
\hline$x$ & 4839261 \\
\hline$x$ & 4840785 \\
\hline$x$ & 4841798 \\
\hline$x$ & 4842343 \\
\hline $\mathrm{X}$ & 4843304 \\
\hline$x$ & 4847002 \\
\hline$x$ & 4847227 \\
\hline$x$ & 4847368 \\
\hline$x$ & 4847701 \\
\hline$x$ & 4847901 \\
\hline $\mathrm{X}$ & 4848057 \\
\hline$x$ & 4857797 \\
\hline$x$ & 4860345 \\
\hline$x$ & 4862763 \\
\hline$x$ & 4863828 \\
\hline$x$ & 4864752 \\
\hline$x$ & 4869234 \\
\hline$x$ & 4873192 \\
\hline$x$ & 4875012 \\
\hline$x$ & 4875332 \\
\hline$x$ & 4875657 \\
\hline$x$ & 4877751 \\
\hline$x$ & 4878600 \\
\hline$x$ & 4878944 \\
\hline$x$ & 4882113 \\
\hline$x$ & 4882668 \\
\hline$x$ & 4882727 \\
\hline$x$ & 4892492 \\
\hline$x$ & 4895958 \\
\hline$x$ & 4897193 \\
\hline$x$ & 4897947 \\
\hline$x$ & 4898999 \\
\hline$x$ & 4901521 \\
\hline$x$ & 4906939 \\
\hline$x$ & 4912620 \\
\hline $\mathrm{X}$ & 4918762 \\
\hline$x$ & 4921109 \\
\hline$x$ & 4927829 \\
\hline$x$ & 4928010 \\
\hline$x$ & 4932058 \\
\hline$x$ & 4933062 \\
\hline$x$ & 4933132 \\
\hline$x$ & 4934609 \\
\hline$x$ & 4935896 \\
\hline$X$ & 4936626 \\
\hline$x$ & 4938395 \\
\hline$x$ & 4938420 \\
\hline
\end{tabular}

\begin{tabular}{|c|c|}
\hline 10 & 32 \\
\hline 10 & 20 \\
\hline 11 & 4 \\
\hline 6 & 11 \\
\hline 4 & 20 \\
\hline 10 & 15 \\
\hline 14 & 8 \\
\hline 15 & 26 \\
\hline 25 & 8 \\
\hline 17 & 33 \\
\hline 9 & 6 \\
\hline 10 & 24 \\
\hline 6 & 24 \\
\hline 5 & 6 \\
\hline 16 & 19 \\
\hline 20 & 7 \\
\hline 6 & 28 \\
\hline 9 & 18 \\
\hline 5 & 10 \\
\hline 4 & 14 \\
\hline 8 & 25 \\
\hline 9 & 24 \\
\hline 4 & 9 \\
\hline 6 & 23 \\
\hline 8 & 15 \\
\hline 15 & 15 \\
\hline 8 & 15 \\
\hline 10 & 10 \\
\hline 6 & 14 \\
\hline 4 & 28 \\
\hline 9 & 22 \\
\hline 5 & 28 \\
\hline 6 & 16 \\
\hline 11 & 22 \\
\hline 6 & 17 \\
\hline 7 & 23 \\
\hline 8 & 23 \\
\hline 5 & 8 \\
\hline 11 & 11 \\
\hline 19 & 10 \\
\hline 10 & 16 \\
\hline 6 & 5 \\
\hline 8 & 25 \\
\hline 5 & 5 \\
\hline 16 & 10 \\
\hline 4 & 25 \\
\hline 13 & 10 \\
\hline 11 & 6 \\
\hline 10 & 12 \\
\hline 8 & 5 \\
\hline 15 & 8 \\
\hline 7 & 15 \\
\hline 5 & 12 \\
\hline
\end{tabular}




\begin{tabular}{|c|c|}
\hline$x$ & 4942575 \\
\hline$x$ & 4946931 \\
\hline$x$ & 4951027 \\
\hline$x$ & 4965559 \\
\hline$x$ & 4976067 \\
\hline$x$ & 4976381 \\
\hline$x$ & 4982668 \\
\hline$x$ & 4983506 \\
\hline$x$ & 4983933 \\
\hline$X$ & 4989352 \\
\hline$X$ & 4989579 \\
\hline$x$ & 5001524 \\
\hline$x$ & 5003599 \\
\hline$x$ & 5005903 \\
\hline$x$ & 5007134 \\
\hline$x$ & 5010924 \\
\hline$x$ & 5011070 \\
\hline$x$ & 5011192 \\
\hline$x$ & 5011932 \\
\hline$x$ & 5012444 \\
\hline$x$ & 5012495 \\
\hline$x$ & 5016273 \\
\hline$x$ & 5018773 \\
\hline$x$ & 5019193 \\
\hline$x$ & 5020319 \\
\hline$x$ & 5020472 \\
\hline$x$ & 5020991 \\
\hline$x$ & 5025901 \\
\hline$x$ & 5026191 \\
\hline$x$ & 5028526 \\
\hline$x$ & 5029140 \\
\hline$x$ & 5031020 \\
\hline$x$ & 5033133 \\
\hline$x$ & 5034060 \\
\hline$x$ & 5034205 \\
\hline$x$ & 5034434 \\
\hline$x$ & 5035722 \\
\hline$x$ & 5038603 \\
\hline$x$ & 5038860 \\
\hline$x$ & 5041026 \\
\hline$x$ & 5045882 \\
\hline$x$ & 5046692 \\
\hline$x$ & 5047749 \\
\hline$x$ & 5048639 \\
\hline$x$ & 5049093 \\
\hline$x$ & 5050075 \\
\hline$x$ & 5052538 \\
\hline$x$ & 5055557 \\
\hline$x$ & 5055592 \\
\hline$x$ & 5055648 \\
\hline$x$ & 5058924 \\
\hline$x$ & 5061367 \\
\hline$x$ & 5070295 \\
\hline
\end{tabular}

\begin{tabular}{|c|c|c|}
\hline 6 & 5 & 0 \\
\hline 6 & 30 & 0 \\
\hline 18 & 7 & 0 \\
\hline 11 & 9 & 0 \\
\hline 12 & 8 & 0 \\
\hline 5 & 6 & 0 \\
\hline 4 & 14 & 0 \\
\hline 22 & 4 & 0 \\
\hline 25 & 7 & 0 \\
\hline 6 & 21 & 0 \\
\hline 9 & 16 & 0 \\
\hline 12 & 16 & 0 \\
\hline 18 & 5 & 0 \\
\hline 4 & 9 & 0 \\
\hline 9 & 7 & 0 \\
\hline 21 & 5 & 0 \\
\hline 17 & 4 & 0 \\
\hline 25 & 7 & 0 \\
\hline 11 & 9 & 0 \\
\hline 13 & 19 & 0 \\
\hline 8 & 10 & 0 \\
\hline 29 & 22 & 0 \\
\hline 4 & 10 & 0 \\
\hline 8 & 9 & 0 \\
\hline 10 & 25 & 0 \\
\hline 6 & 16 & 0 \\
\hline 5 & 9 & 0 \\
\hline 4 & 17 & 0 \\
\hline 4 & 13 & 0 \\
\hline 17 & 9 & 0 \\
\hline 9 & 19 & 0 \\
\hline 18 & 27 & 0 \\
\hline 6 & 11 & 0 \\
\hline 5 & 18 & 0 \\
\hline 7 & 12 & 0 \\
\hline 4 & 14 & 0 \\
\hline 11 & 11 & 0 \\
\hline 15 & 6 & 0 \\
\hline 6 & 13 & 0 \\
\hline 4 & 12 & 0 \\
\hline 13 & 7 & 0 \\
\hline 16 & 7 & 0 \\
\hline 7 & 18 & 0 \\
\hline 7 & 20 & 0 \\
\hline 5 & 11 & 0 \\
\hline 5 & 6 & 0 \\
\hline 24 & 4 & 0 \\
\hline 7 & 7 & 0 \\
\hline 5 & 15 & 0 \\
\hline 4 & 20 & 0 \\
\hline 13 & 5 & 0 \\
\hline 7 & 25 & 0 \\
\hline 5 & 25 & 0 \\
\hline
\end{tabular}




\begin{tabular}{|c|c|}
\hline$x$ & 5071111 \\
\hline$x$ & 5072123 \\
\hline$X$ & 5072191 \\
\hline$x$ & 5073593 \\
\hline$x$ & 5075686 \\
\hline$x$ & 5076275 \\
\hline$x$ & 5077138 \\
\hline$x$ & 5078718 \\
\hline$x$ & 5078823 \\
\hline$x$ & 5078933 \\
\hline$x$ & 5079091 \\
\hline$x$ & 5085992 \\
\hline$x$ & 5088298 \\
\hline$x$ & 5091924 \\
\hline$x$ & 5092301 \\
\hline$x$ & 5092610 \\
\hline$x$ & 5093625 \\
\hline$x$ & 5093904 \\
\hline$x$ & 5095364 \\
\hline$x$ & 5096566 \\
\hline$x$ & 5096943 \\
\hline$x$ & 5098901 \\
\hline$x$ & 5099856 \\
\hline$x$ & 5100067 \\
\hline$x$ & 5100234 \\
\hline$x$ & 5101304 \\
\hline$x$ & 5103133 \\
\hline$x$ & 5109228 \\
\hline$x$ & 5110329 \\
\hline$x$ & 5110343 \\
\hline$x$ & 5110911 \\
\hline$x$ & 5110920 \\
\hline$x$ & 5111192 \\
\hline$x$ & 5111376 \\
\hline$x$ & 5114751 \\
\hline$x$ & 5116099 \\
\hline$x$ & 5116910 \\
\hline$x$ & 5117227 \\
\hline$x$ & 5117274 \\
\hline$x$ & 5119409 \\
\hline$x$ & 5123520 \\
\hline$x$ & 5130418 \\
\hline$x$ & 5130455 \\
\hline$x$ & 5139452 \\
\hline$x$ & 5140268 \\
\hline$x$ & 5145254 \\
\hline$x$ & 5148120 \\
\hline$x$ & 5151720 \\
\hline$x$ & 5153106 \\
\hline$x$ & 5153873 \\
\hline$x$ & 5154001 \\
\hline$x$ & 5154676 \\
\hline$x$ & 5155639 \\
\hline
\end{tabular}

\section{2}

2

2

2

2

2

2

2

2

2

2

2

2

2

2

2

2

2

2

2

2

2

2

2

2

2

2

2

2

2

2

2

2

2

2

2

2

2

2

2

2

2

2

2

2

2

2

2

2

2

2

2

2
7

12

9

8
15

11

5

5

7

11

15

27

14

14

8

7

6
12

12

23

11

18

7

9

5

6

18

15

4

16

17

11

8

21

25

16

5

10

10

9

11

5

21

20

13

22

5

4

15

8

8

8

21
14

17

29

12

5

6

13

23

17

21

10

6

15

9

24

14

5

16

23

6

8

26

7

6

7

8

15

9

6

6

16

17

11

15

15

6

7

19

16

14

14

5

4

9

8

26

21

29

11

7

22

12

20
0

0

0

0

0

0

0

0

0

0

0

0

0

0

0

0

0

0

0

0

0

0

0

0

0

0

0

0

0

0

0

0

0

0

0

0

0

0

0

0

0

0

0

0

0

0

0

0

0 


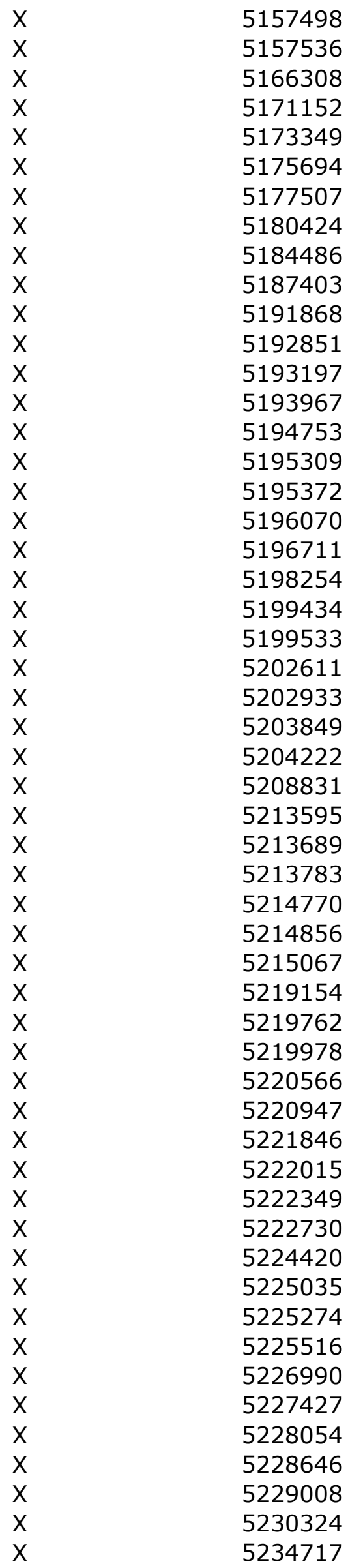

\begin{tabular}{|c|c|}
\hline 2 & 5 \\
\hline 2 & 17 \\
\hline 2 & 9 \\
\hline 2 & 11 \\
\hline 2 & 7 \\
\hline 2 & 11 \\
\hline 2 & 9 \\
\hline 2 & 22 \\
\hline 2 & 12 \\
\hline 2 & 5 \\
\hline 2 & 23 \\
\hline 2 & 12 \\
\hline 2 & 19 \\
\hline 2 & 10 \\
\hline 2 & 11 \\
\hline 2 & 14 \\
\hline 2 & 18 \\
\hline 2 & 10 \\
\hline 2 & 19 \\
\hline 2 & 18 \\
\hline 2 & 8 \\
\hline 2 & 6 \\
\hline 2 & 20 \\
\hline 2 & 21 \\
\hline 2 & 12 \\
\hline 2 & 15 \\
\hline 2 & 19 \\
\hline 2 & 16 \\
\hline 2 & 19 \\
\hline 2 & 26 \\
\hline 2 & 21 \\
\hline 2 & 14 \\
\hline 2 & 10 \\
\hline 2 & 13 \\
\hline 2 & 4 \\
\hline 2 & 10 \\
\hline 2 & 13 \\
\hline 2 & 25 \\
\hline 2 & 13 \\
\hline 2 & 17 \\
\hline 2 & 5 \\
\hline 2 & 4 \\
\hline 2 & 15 \\
\hline 2 & 16 \\
\hline 2 & 15 \\
\hline 2 & 24 \\
\hline 2 & 13 \\
\hline 2 & 32 \\
\hline 2 & 27 \\
\hline 2 & 4 \\
\hline 2 & 19 \\
\hline 2 & 14 \\
\hline 2 & \\
\hline
\end{tabular}

17

17

11

8

5

8

19

9

5

5

14

13

13

4

6

9

15

7

15

13

7

20

7

12

4

19

7

8

6

5

5

4

19

4

12

5

8

24

11

4

7

10

4

7

11

36

14

21

14

12

8

4

26
0

0

0

0

0

0

0

0

0

0

0

0

0

0

0

0

0

0

0

0

0

0

0

0

0

0

0

0

0

0

0

0

0

0

0

0

0

0

0

0

0

0

0

0

0

0

0

0

0

0

0 


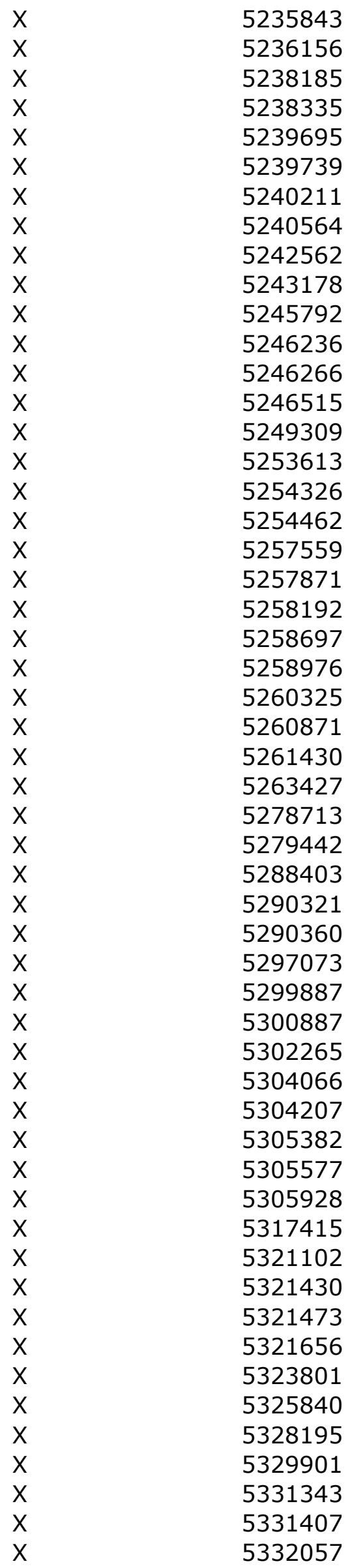

\begin{tabular}{|c|c|c|c|}
\hline 2 & 9 & 4 & 0 \\
\hline 2 & 4 & 22 & 0 \\
\hline 2 & 16 & 6 & 0 \\
\hline 2 & 11 & 15 & 0 \\
\hline 2 & 18 & 4 & 0 \\
\hline 2 & 14 & 6 & 0 \\
\hline 2 & 14 & 10 & 0 \\
\hline 2 & 19 & 7 & 0 \\
\hline 2 & 9 & 6 & 0 \\
\hline 2 & 8 & 26 & 0 \\
\hline 2 & 12 & 7 & 0 \\
\hline 2 & 15 & 4 & 0 \\
\hline 2 & 14 & 15 & 0 \\
\hline 2 & 10 & 4 & 0 \\
\hline 2 & 17 & 23 & 0 \\
\hline 2 & 14 & 8 & 0 \\
\hline 2 & 8 & 19 & 0 \\
\hline 2 & 6 & 12 & 0 \\
\hline 2 & 18 & 7 & 0 \\
\hline 2 & 19 & 16 & 0 \\
\hline 2 & 11 & 9 & 0 \\
\hline 2 & 4 & 20 & 0 \\
\hline 2 & 13 & 8 & 0 \\
\hline 2 & 13 & 10 & 0 \\
\hline 2 & 12 & 6 & 0 \\
\hline 2 & 18 & 10 & 0 \\
\hline 2 & 20 & 4 & 0 \\
\hline 2 & 11 & 5 & 0 \\
\hline 2 & 8 & 9 & 0 \\
\hline 2 & 18 & 4 & 0 \\
\hline 2 & 21 & 4 & 0 \\
\hline 2 & 10 & 7 & 0 \\
\hline 2 & 14 & 11 & 0 \\
\hline 2 & 4 & 20 & 0 \\
\hline 2 & 11 & 30 & 0 \\
\hline 2 & 5 & 8 & 0 \\
\hline 2 & 29 & 17 & 0 \\
\hline 2 & 5 & 16 & 0 \\
\hline 2 & 6 & 18 & 0 \\
\hline 2 & 5 & 8 & 0 \\
\hline 2 & 22 & 14 & 0 \\
\hline 2 & 5 & 16 & 0 \\
\hline 2 & 11 & 19 & 0 \\
\hline 2 & 20 & 13 & 0 \\
\hline 2 & 15 & 14 & 0 \\
\hline 2 & 6 & 11 & 0 \\
\hline 2 & 5 & 5 & 0 \\
\hline 2 & 9 & 22 & 0 \\
\hline 2 & 7 & 7 & 0 \\
\hline 2 & 12 & 5 & 0 \\
\hline 2 & 6 & 14 & 0 \\
\hline 2 & 12 & 4 & 0 \\
\hline 2 & 25 & 9 & 0 \\
\hline
\end{tabular}




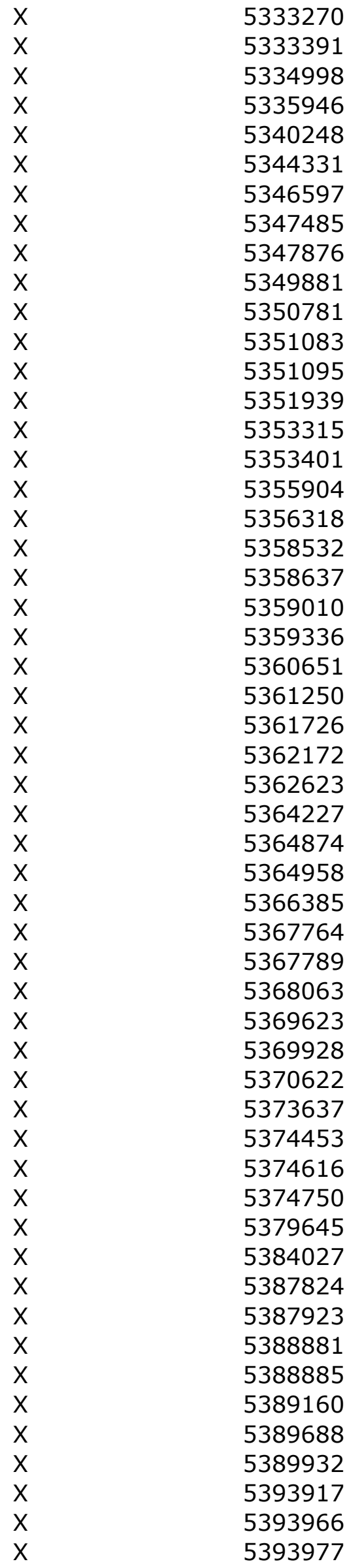

2
2
2
2
2
2
2
2
2
2
2
2
2
2
2
2
2
2
2
2
2
2
2
2
2
2
2
2
2
2
2
2
2
2
2
2
2
2
2
2
2
2
2
2
2
2
2
2
2
2
2

$\begin{array}{rr}4 & 15 \\ 11 & \\ 17 & \\ 6 & 1 \\ 17 & 13 \\ 9 & \end{array}$

15

6

0

2

7

16

4

31

17

11

13

9

25

25

8

6

9

14

12

8

11

14

16

17

6

10

12

13

8 


\begin{tabular}{|c|c|}
\hline$x$ & 5396401 \\
\hline$x$ & 5399011 \\
\hline$x$ & 5403237 \\
\hline$x$ & 5404242 \\
\hline$x$ & 5405229 \\
\hline$X$ & 5405601 \\
\hline$X$ & 5407045 \\
\hline$x$ & 5407202 \\
\hline$x$ & 5407263 \\
\hline$x$ & 5407285 \\
\hline$x$ & 5408214 \\
\hline$x$ & 5424670 \\
\hline$x$ & 5425087 \\
\hline$X$ & 5425231 \\
\hline$x$ & 5426393 \\
\hline$x$ & 5427463 \\
\hline$x$ & 5429721 \\
\hline$x$ & 5430082 \\
\hline$x$ & 5432239 \\
\hline$x$ & 5433293 \\
\hline$x$ & 5436033 \\
\hline$x$ & 5436562 \\
\hline$x$ & 5437356 \\
\hline$x$ & 5437475 \\
\hline$x$ & 5440743 \\
\hline$x$ & 5441216 \\
\hline$X$ & 5442777 \\
\hline$x$ & 5443393 \\
\hline$x$ & 5443743 \\
\hline$x$ & 5443782 \\
\hline$x$ & 5444182 \\
\hline$x$ & 5446282 \\
\hline$x$ & 5446886 \\
\hline$x$ & 5448058 \\
\hline$x$ & 5448291 \\
\hline$x$ & 5449042 \\
\hline$x$ & 5449276 \\
\hline$x$ & 5451550 \\
\hline$x$ & 5453092 \\
\hline$X$ & 5454152 \\
\hline$x$ & 5454820 \\
\hline$x$ & 5455449 \\
\hline$x$ & 5455705 \\
\hline$x$ & 5458931 \\
\hline$x$ & 5460308 \\
\hline$X$ & 5460916 \\
\hline$x$ & 5464309 \\
\hline$x$ & 5464386 \\
\hline$x$ & 5467731 \\
\hline$x$ & 5467811 \\
\hline$X$ & 5467912 \\
\hline$x$ & 5469879 \\
\hline$x$ & 5470476 \\
\hline
\end{tabular}

14

11

13

10

13

10

18

16

6

17

14

20

7

4

8

28

11

13

10

6

6

17

7

17

6

7

8

6

19

4

12

25

8

18

5

15

12

21

12

13

11

12

14

13

4

14

10

7

4

5

4

8

12

$\begin{array}{rl}5 & 0 \\ 11 & 0 \\ 15 & 0 \\ 7 & 0 \\ 5 & 0 \\ 5 & 0 \\ 9 & 0 \\ 5 & 0 \\ 8 & 0 \\ 9 & 0 \\ 22 & 0 \\ 16 & 0 \\ 16 & 0 \\ 14 & 0 \\ 9 & 0 \\ 5 & 0 \\ 4 & 0 \\ 19 & 0 \\ 9 & 0\end{array}$

17

11

11

20

19

13

6

12

10

20

24

12

17

8

10

15

9

18

13

16

4

25

4

18

5

11

4

5

9

18

19

9

10

6
0

0

0

0

0

0

0

0

0

0

0

0

0

0

0

0

0

0

0

0

0

0

0

0

0

0

0

0

0

0

0

0

0

0

0

0

0

0

0

0

0

0

0 
$x$
$x$

5470669

5470909

5472752

5473552

5473714

5478022

5478266

5482809

5483839

5484309

5484391

5486381

5487232

5487286

5488175

5489730

5490353

5490456

5490556

5493329

5496494

5508297

5508669

5511053

5512137

5516061

5518663

5518762

5518864

5521044

5521545

5522538

5522954

5523343

5523941

5523999

5524632

5526703

5527662

5527896

5528728

5528769

5530181

5531402

5532992

5534414

5535222

5535453

5535512

5535660

5535738

5536506

5536823
2

2

2

2

2

2

2

2

2

2

2

2

2

2

2

2

2

2

2

2

2

2

2

2

2

2

2

2

2

2

2

2

2

2

2

2

2

2

2

2

2

2

2

2

2

2

2

2

2

2

2

2

2
8
8
30
16
12
8
6
5

12

11

17

5

8

6

8

7

5

13

20

27

14

17

5

9

25

26

10

12

5

12

6

17

5

19

5

5

13

12

5

6

17

21

14

11

5

16

4

11

12

10

10

5

15

$\begin{array}{rl}8 & 0 \\ 5 & 0 \\ 16 & 0 \\ 23 & 0 \\ 11 & 0 \\ 7 & 0 \\ 18 & 0 \\ 17 & 0 \\ 4 & 0 \\ 5 & 0 \\ 7 & 0 \\ 6 & 0 \\ 13 & 0 \\ 15 & 0 \\ 5 & 0 \\ 15 & 0 \\ 7 & 0\end{array}$

$13 \quad 0$

$16 \quad 0$

$10 \quad 0$

$4 \quad 0$

40

$6 \quad 0$

$6 \quad 0$

$10 \quad 0$

15

35

9

11

37

20

5

10

34

28

17

18

4

8

22

5

4

22

27

16

9

20

24

23

30

13

19

10
0

0

0

0

0

0

0

0

0

0

0

0

0

0

0

0

0

0

0

0

0

0

0

0

0
0 


\begin{tabular}{|c|c|}
\hline$x$ & 5537115 \\
\hline$x$ & 5539174 \\
\hline$x$ & 5539253 \\
\hline$x$ & 5539390 \\
\hline$x$ & 5539428 \\
\hline$x$ & 5546524 \\
\hline $\mathrm{X}$ & 5546944 \\
\hline $\mathrm{X}$ & 5548937 \\
\hline$x$ & 5549060 \\
\hline$x$ & 5549562 \\
\hline$x$ & 5549650 \\
\hline$x$ & 5550130 \\
\hline$x$ & 5550241 \\
\hline$x$ & 5552624 \\
\hline$x$ & 5552734 \\
\hline$x$ & 5553016 \\
\hline$x$ & 5553373 \\
\hline$x$ & 5553815 \\
\hline$x$ & 5554973 \\
\hline $\mathrm{X}$ & 5556124 \\
\hline$x$ & 5557195 \\
\hline$x$ & 5557350 \\
\hline$x$ & 5558237 \\
\hline$x$ & 5558588 \\
\hline$x$ & 5559011 \\
\hline$x$ & 5563355 \\
\hline$x$ & 5565162 \\
\hline$X$ & 5565195 \\
\hline$x$ & 5565571 \\
\hline$x$ & 5565906 \\
\hline$x$ & 5570153 \\
\hline $\mathrm{X}$ & 5570457 \\
\hline$x$ & 5570517 \\
\hline$x$ & 5570557 \\
\hline$x$ & 5570569 \\
\hline$x$ & 5570659 \\
\hline$x$ & 5570863 \\
\hline$x$ & 5570935 \\
\hline$x$ & 5570984 \\
\hline$x$ & 5571097 \\
\hline$x$ & 5571299 \\
\hline$x$ & 5571880 \\
\hline$x$ & 5572426 \\
\hline$x$ & 5574799 \\
\hline$x$ & 5575696 \\
\hline$x$ & 5575986 \\
\hline$x$ & 5576069 \\
\hline$x$ & 5576816 \\
\hline$x$ & 5578049 \\
\hline$x$ & 5578177 \\
\hline$x$ & 5579407 \\
\hline$x$ & 5579952 \\
\hline$X$ & 5580182 \\
\hline
\end{tabular}

$\begin{array}{rr}5 & 1 \\ 8 & \\ 8 & 2 \\ 5 & 15 \\ 9 & 25 \\ 15 & 18 \\ 12 & \end{array}$

$14 \quad 0$

8

22

15

25

18

4

18

18

15

28

16

13

12

34

26

14

7

7

18

8

12

8

17

15

5

6

3

14

9

13

13

14

9

23

15

14

16

10

15

17

26

27

12

6

13

5

13

9

12 


\begin{tabular}{|c|c|}
\hline$x$ & 5580790 \\
\hline$x$ & 5581555 \\
\hline$X$ & 5583221 \\
\hline$x$ & 5585576 \\
\hline$x$ & 5586205 \\
\hline$X$ & 5586349 \\
\hline$x$ & 5588120 \\
\hline$X$ & 5588192 \\
\hline$X$ & 5588504 \\
\hline$x$ & 5588570 \\
\hline$X$ & 5588757 \\
\hline$x$ & 5593044 \\
\hline$x$ & 5594000 \\
\hline$x$ & 5596754 \\
\hline$x$ & 5597490 \\
\hline$X$ & 5597665 \\
\hline$x$ & 5599063 \\
\hline$x$ & 5601995 \\
\hline$x$ & 5603594 \\
\hline$x$ & 5603789 \\
\hline$x$ & 5607727 \\
\hline$x$ & 5608795 \\
\hline$x$ & 5609107 \\
\hline$x$ & 5609376 \\
\hline$x$ & 5613557 \\
\hline$x$ & 5614337 \\
\hline$X$ & 5614342 \\
\hline$x$ & 5614549 \\
\hline$x$ & 5615630 \\
\hline$X$ & 5616347 \\
\hline$x$ & 5616434 \\
\hline$x$ & 5618524 \\
\hline$x$ & 5618629 \\
\hline$X$ & 5619924 \\
\hline$x$ & 5620845 \\
\hline$X$ & 5627032 \\
\hline$x$ & 5627107 \\
\hline$x$ & 5627239 \\
\hline$x$ & 5628473 \\
\hline$x$ & 5629429 \\
\hline$X$ & 5629580 \\
\hline$X$ & 5632252 \\
\hline$X$ & 5633055 \\
\hline$x$ & 5646317 \\
\hline$X$ & 5646442 \\
\hline$x$ & 5646693 \\
\hline$x$ & 5650919 \\
\hline$X$ & 5653155 \\
\hline$x$ & 5653547 \\
\hline$x$ & 5653894 \\
\hline$x$ & 5654343 \\
\hline$x$ & 5655751 \\
\hline$x$ & 5656 \\
\hline
\end{tabular}

$2 \quad 19$

19
10

6

0

210

13

18

13

18

17

35

16

13

14

6

12

14

6

22

21

13

7

8

5

8

21

10

22

17

18

15

7

7
18

17

6

12

16

15

11

12

18

26

10

12

15

10

11

13

11

16

18

5

8

9

9

11

4

10

10

4

12

7

14

6

9

5

8

11

16

25

12

13

18

25

11

13

18

16

7

16

$\begin{array}{ll}2 & 17 \\ 2 & 13\end{array}$

$\begin{array}{ll}2 & 17 \\ 2 & 13\end{array}$

9

4

5

6

10

4

4

6

17

11

16

15

4

6

0

0

0

0

0

0

0

0

0

0

0

0

0

0

0

0

0

0

0

0

0

0

0

0

0

0

0

0

0

0

0

0

0

0

0

0

0

0

0

0

0 


\begin{tabular}{|c|c|}
\hline$x$ & 5661340 \\
\hline$x$ & 5661524 \\
\hline$x$ & 5665905 \\
\hline$x$ & 5666875 \\
\hline$x$ & 5669091 \\
\hline$x$ & 5670273 \\
\hline$x$ & 5670490 \\
\hline$x$ & 5671757 \\
\hline$x$ & 5673356 \\
\hline$x$ & 5679990 \\
\hline$x$ & 5688207 \\
\hline$x$ & 5690823 \\
\hline$x$ & 5693170 \\
\hline$x$ & 5693481 \\
\hline$x$ & 5694650 \\
\hline$x$ & 5696439 \\
\hline$x$ & 5697955 \\
\hline$x$ & 5700584 \\
\hline$x$ & 5700653 \\
\hline$x$ & 5702342 \\
\hline$x$ & 5702660 \\
\hline$x$ & 5702778 \\
\hline$x$ & 5703221 \\
\hline$x$ & 5703248 \\
\hline$x$ & 5703508 \\
\hline$x$ & 5709316 \\
\hline$x$ & 5709736 \\
\hline$x$ & 5709829 \\
\hline$x$ & 5709889 \\
\hline$x$ & 5710066 \\
\hline$x$ & 5710567 \\
\hline$x$ & 5710688 \\
\hline$x$ & 5710783 \\
\hline$x$ & 5713700 \\
\hline$x$ & 5714393 \\
\hline$x$ & 5714993 \\
\hline$x$ & 5715556 \\
\hline$x$ & 5716171 \\
\hline$x$ & 5716480 \\
\hline$X$ & 5716933 \\
\hline$X$ & 5717154 \\
\hline$X$ & 5718460 \\
\hline$X$ & 5719552 \\
\hline$x$ & 5720883 \\
\hline$X$ & 5721290 \\
\hline$X$ & 5722257 \\
\hline$X$ & 5722826 \\
\hline$x$ & 5724450 \\
\hline$X$ & 5724634 \\
\hline$X$ & 5726906 \\
\hline$X$ & 5727198 \\
\hline$X$ & 5729317 \\
\hline $\mathrm{x}$ & 5729979 \\
\hline
\end{tabular}

32

23

8

30

6

12

13

5

9

11

42

9

19

21

29

17

13

13

9

27

10

7

6

11

16

6

7

6

13

5

13

6

19

14

10

5

4

12

5

10

8

6

17

12

6

5

20

8

9

4

4

12

14
21

15

15

13

11

13

13

12

9

8

10

11

8

10

5

8

6

22

13

13

19

10

23

31

19

13

21

5

29

19

5

8

10

7

10

18

11

6

15

13

24

9

5

9

26

10

33

19

26

14

27

23

14
0

0

0

0

0

0

0

0

0

0

0

0

0

0

0

0

0

0

0

0

0

0

0

0

0

0

0

0

0

0

0

0

0

0

0

0

0

0

0

0

0

0

0

0

0

0

0

0

0 


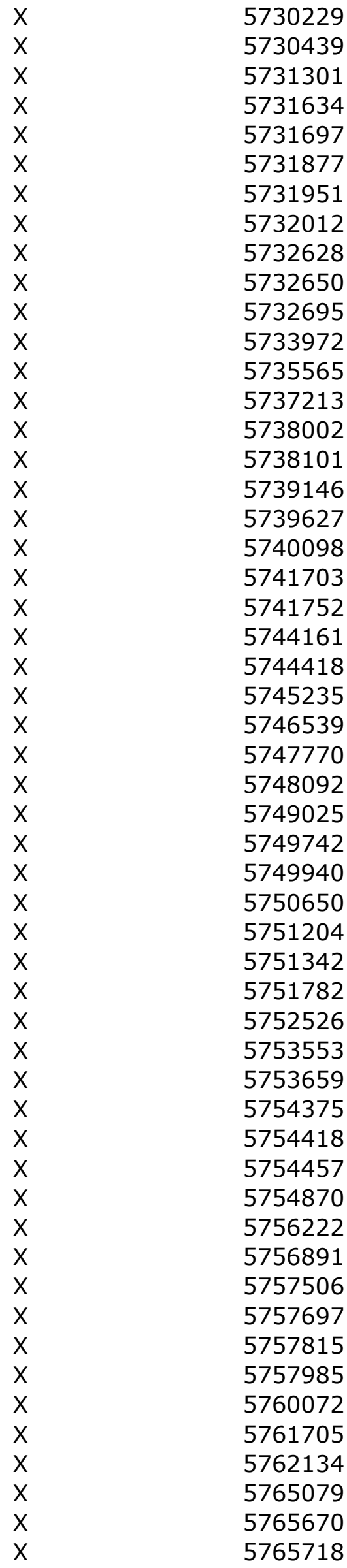

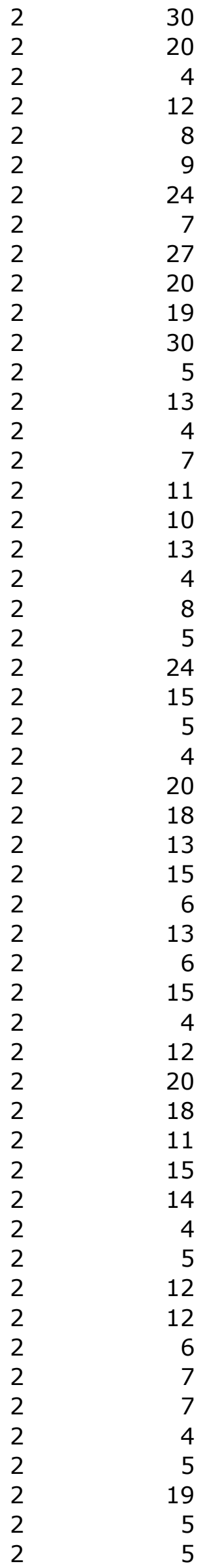

16

12

12

21

18

8

10

10

10

6

12

10

28

8

13

17

4

14

4

11

14

26

11

12

20

15

14

8

7

5

8

5

9

17

13

13

4

24

30

32

9

12

9

10

14

10

12

20

24

10

21

17

14
0

0

0

0

0

0

0

0

0

0

0

0

0

0

0

0

0

0

0

0

0

0

0

0

0

0

0

0

0

0

0

0

0

0

0

0

0

0

0

0

0

0

0

0

0

0

0

0

0

0

0 


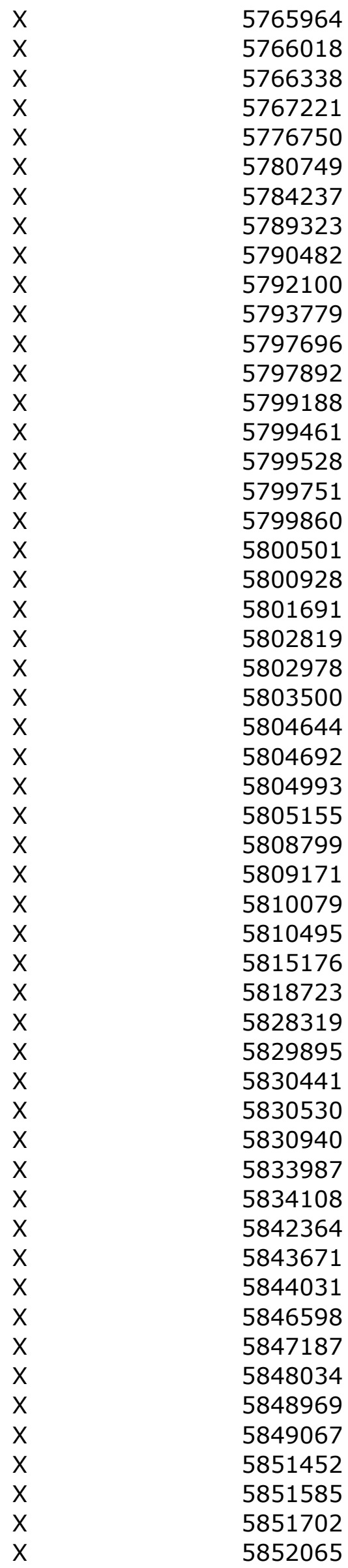

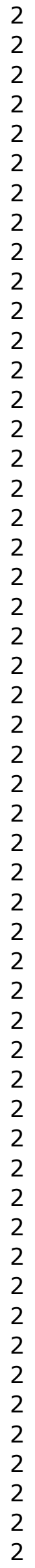

7
5
7
6
5
10
17
13
20

17

30

10

12

8

31

11

13

20

18

16

19

16

413

$19 \quad 18$

$7 \quad 11$

$\begin{array}{ll}7 & 19\end{array}$

$4 \quad 17$

$17 \quad 29$

5

4

11

5

16

21

22

25

9

10

7

5

10

11

0<smiles>[O]</smiles>$$
0
$$$$
0
$$$$
0
$$$$
0
$$$$
0
$$$$
0
$$$$
0
$$$$
0
$$$$
0
$$$$
0
$$$$
0
$$$$
0
$$$$
0
$$$$
0
$$$$
0
$$$$
0
$$$$
0
$$$$
0
$$$$
0
$$$$
0
$$$$
0
$$$$
0
$$$$
0
$$$$
0
$$$$
0
$$$$
0
$$$$
0
$$$$
0
$$$$
0
$$$$
0
$$$$
0
$$$$
0
$$$$
0
$$$$
0
$$$$
0
$$$$
0
$$$$
0
$$$$
0
$$$$
0
$$$$
\begin{aligned}
& 0 \\
& 0
\end{aligned}
$$$$
0
$$$$
0
$$$$
0
$$$$
0
$$ 


\begin{tabular}{|c|c|}
\hline$x$ & 5852139 \\
\hline$x$ & 5852398 \\
\hline$x$ & 5852482 \\
\hline$x$ & 5852573 \\
\hline$x$ & 5852671 \\
\hline $\mathrm{X}$ & 5852866 \\
\hline$x$ & 5852893 \\
\hline$x$ & 5852956 \\
\hline$x$ & 5853115 \\
\hline$x$ & 5853718 \\
\hline$x$ & 5853955 \\
\hline$x$ & 5853997 \\
\hline$x$ & 5854417 \\
\hline$x$ & 5854445 \\
\hline$x$ & 5854809 \\
\hline$x$ & 5854842 \\
\hline$x$ & 5854980 \\
\hline$x$ & 5855032 \\
\hline$x$ & 5855248 \\
\hline$x$ & 5855323 \\
\hline$x$ & 5855398 \\
\hline$x$ & 5855488 \\
\hline$x$ & 5855639 \\
\hline$x$ & 5855699 \\
\hline$x$ & 5856938 \\
\hline$x$ & 5856975 \\
\hline$x$ & 5858071 \\
\hline$x$ & 5858267 \\
\hline$x$ & 5859380 \\
\hline$x$ & 5862194 \\
\hline$x$ & 5862903 \\
\hline$x$ & 5864086 \\
\hline$x$ & 5866486 \\
\hline$x$ & 5867190 \\
\hline$x$ & 5867527 \\
\hline$x$ & 5867578 \\
\hline$x$ & 5869060 \\
\hline$X$ & 5869442 \\
\hline$x$ & 5869791 \\
\hline$x$ & 5869989 \\
\hline$x$ & 5870258 \\
\hline$x$ & 5872711 \\
\hline $\mathrm{X}$ & 5876778 \\
\hline$x$ & 5878203 \\
\hline$x$ & 5878369 \\
\hline$x$ & 5878622 \\
\hline$x$ & 5878694 \\
\hline$x$ & 5878721 \\
\hline$x$ & 5879133 \\
\hline$x$ & 5879270 \\
\hline$x$ & 5879354 \\
\hline$x$ & 5880044 \\
\hline$x$ & 5880212 \\
\hline
\end{tabular}

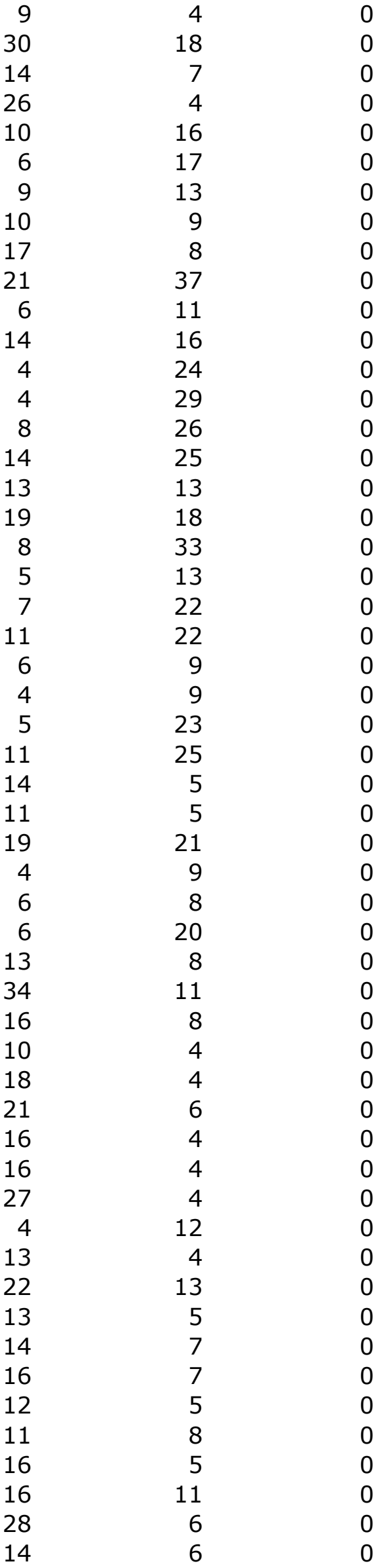




\begin{tabular}{|c|c|}
\hline$x$ & 5880239 \\
\hline$x$ & 5880577 \\
\hline$x$ & 5880594 \\
\hline$x$ & 5880610 \\
\hline$x$ & 5882438 \\
\hline$x$ & 5882845 \\
\hline$x$ & 5885715 \\
\hline$x$ & 5886052 \\
\hline$x$ & 5886133 \\
\hline$x$ & 5888363 \\
\hline$x$ & 5888484 \\
\hline$X$ & 5889724 \\
\hline$x$ & 5891109 \\
\hline$x$ & 5894257 \\
\hline$x$ & 5895081 \\
\hline$x$ & 5895244 \\
\hline$x$ & 5897562 \\
\hline$x$ & 6119956 \\
\hline$x$ & 6168843 \\
\hline$x$ & 6465592 \\
\hline$x$ & 6571844 \\
\hline$x$ & 6572395 \\
\hline$x$ & 6575773 \\
\hline$x$ & 6575800 \\
\hline$x$ & 6582487 \\
\hline$x$ & 6583757 \\
\hline$x$ & 6585289 \\
\hline$x$ & 6593878 \\
\hline$x$ & 6597739 \\
\hline$x$ & 6598318 \\
\hline$x$ & 6600512 \\
\hline$x$ & 6601533 \\
\hline$x$ & 6601767 \\
\hline$x$ & 6603468 \\
\hline$x$ & 6604261 \\
\hline$x$ & 6604946 \\
\hline$x$ & 6606180 \\
\hline$x$ & 6606813 \\
\hline$x$ & 6608733 \\
\hline$x$ & 6609190 \\
\hline$x$ & 6610012 \\
\hline$x$ & 6610574 \\
\hline$x$ & 6611198 \\
\hline$x$ & 6611424 \\
\hline$x$ & 6611973 \\
\hline$x$ & 6612164 \\
\hline$x$ & 6612183 \\
\hline$x$ & 6612418 \\
\hline$x$ & 6613724 \\
\hline$x$ & 6613791 \\
\hline$x$ & 6613900 \\
\hline$x$ & 6615486 \\
\hline$x$ & 6618395 \\
\hline
\end{tabular}

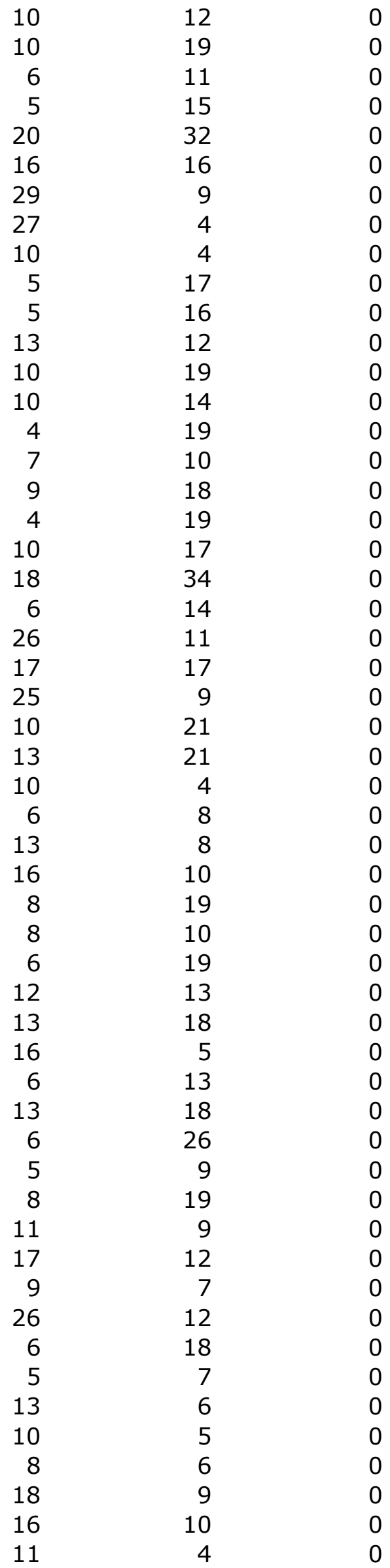




\begin{tabular}{|c|c|}
\hline$x$ & 6618940 \\
\hline$x$ & 6619041 \\
\hline$X$ & 6619085 \\
\hline$x$ & 6619362 \\
\hline$x$ & 6619933 \\
\hline$X$ & 6620581 \\
\hline$x$ & 6620645 \\
\hline$X$ & 6620964 \\
\hline$x$ & 6621894 \\
\hline$x$ & 6622969 \\
\hline$X$ & 6625195 \\
\hline$x$ & 6625387 \\
\hline$x$ & 6625440 \\
\hline$x$ & 6626796 \\
\hline$X$ & 6626821 \\
\hline$x$ & 6628403 \\
\hline$x$ & 6628753 \\
\hline$x$ & 6628776 \\
\hline$X$ & 6628918 \\
\hline$x$ & 6629830 \\
\hline$x$ & 6630375 \\
\hline$x$ & 6631402 \\
\hline$x$ & 6632680 \\
\hline$x$ & 6635566 \\
\hline$x$ & 6636974 \\
\hline$x$ & 6638982 \\
\hline$X$ & 6639441 \\
\hline$x$ & 6639606 \\
\hline$x$ & 6640035 \\
\hline$X$ & 6640052 \\
\hline$x$ & 6640218 \\
\hline$x$ & 6640671 \\
\hline$x$ & 6644439 \\
\hline$x$ & 6644566 \\
\hline$x$ & 6646564 \\
\hline$X$ & 6647788 \\
\hline$x$ & 6647844 \\
\hline$x$ & 6647893 \\
\hline$x$ & 6648750 \\
\hline$x$ & 6655602 \\
\hline$X$ & 6657921 \\
\hline$x$ & 6658333 \\
\hline$X$ & 6660590 \\
\hline$x$ & 6660915 \\
\hline$X$ & 6661164 \\
\hline$x$ & 6664242 \\
\hline$x$ & 6665700 \\
\hline$x$ & 6665972 \\
\hline$x$ & 6666218 \\
\hline$x$ & 6666564 \\
\hline$x$ & 6666776 \\
\hline$x$ & 6667122 \\
\hline$x$ & 6667405 \\
\hline
\end{tabular}

$2 \quad 21$

21

21

20

12

10

6

9

4

9

5

6

23

16

5

15

10

13

9

10

10

13

21

4

4

14

16

5

33

26

13

6

7

8

11

11

31

8

6

12

5

18

6

6

28

8

6

14

20

17

19

19
13

212
$8 \quad 0$

$15 \quad 0$

11

23

5

5

16

16

16

16

13

17

7

13

11

5

5

4

30

15

5

4

8

16

14

19

8

5

6

4

14

29

5

12

7

4

18

10

16

13

14

17

9

13

20

7

11

6

13

18

13

5

9

0

$$
0
$$$$
0
$$$$
0
$$$$
0
$$$$
0
$$$$
0
$$$$
0
$$$$
0
$$$$
\begin{aligned}
& 0 \\
& 0
\end{aligned}
$$$$
0
$$$$
0
$$$$
0
$$$$
0
$$$$
0
$$$$
0
$$$$
0
$$$$
0
$$$$
0
$$$$
0
$$$$
0
$$$$
0
$$$$
0
$$$$
0
$$$$
0
$$$$
0
$$$$
0
$$$$
0
$$$$
0
$$$$
0
$$$$
0
$$$$
0
$$$$
0
$$$$
0
$$$$
0
$$$$
0
$$$$
0
$$$$
0
$$$$
\begin{aligned}
& 0 \\
& 0
\end{aligned}
$$$$
0
$$$$
\begin{aligned}
& 0 \\
& 0
\end{aligned}
$$$$
\begin{aligned}
& 0 \\
& 0
\end{aligned}
$$ 


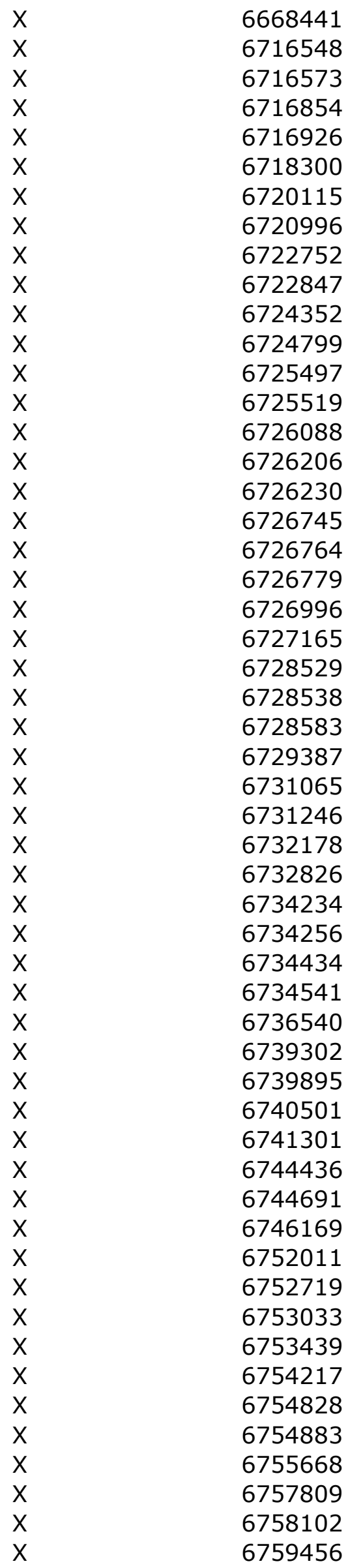

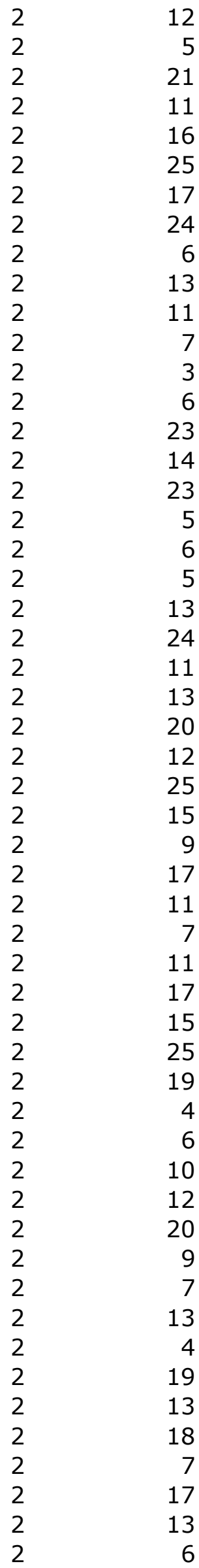

12

14

21

5

0

16

25

17

24

13

11

9

0

15

13

9

7

11

23

18

35

32

6

4

17

6

5

12

4

19
15

15

14

6

5

16

9

17

4

6

20

16

12

17

18

16

16

10

6

26

22

13

5

13

10

16

9

4

19

12

5

14

$$
0
$$$$
0
$$$$
0
$$$$
0
$$$$
0
$$$$
0
$$$$
0
$$$$
0
$$$$
0
$$$$
0
$$$$
0
$$$$
0
$$$$
0
$$$$
0
$$$$
0
$$$$
0
$$$$
0
$$$$
0
$$$$
0
$$$$
0
$$$$
0
$$$$
0
$$$$
0
$$$$
0
$$$$
0
$$$$
0
$$$$
0
$$$$
0
$$$$
0
$$$$
0
$$$$
0
$$$$
0
$$$$
0
$$$$
0
$$$$
0
$$$$
0
$$

19

18

7
17

13
6 


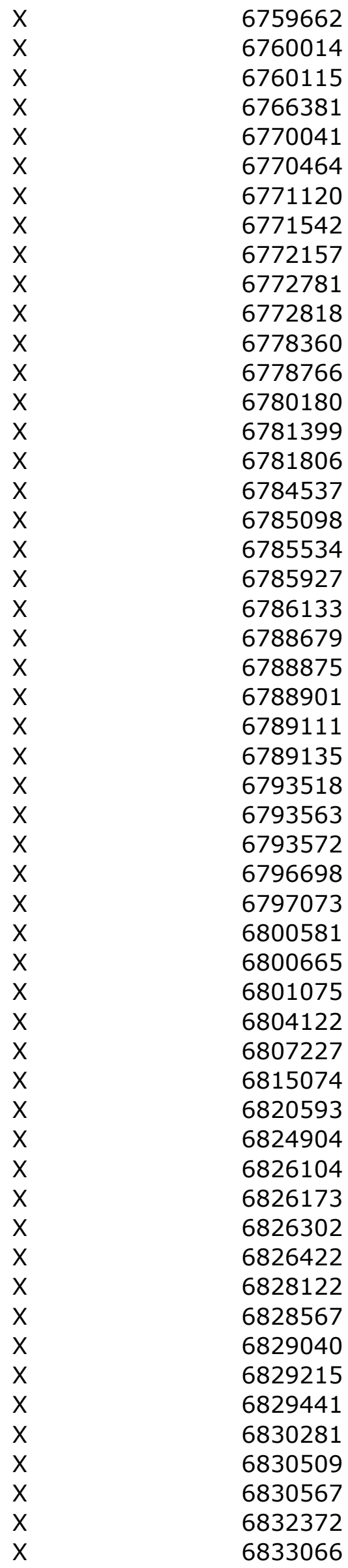

\begin{tabular}{|c|c|c|c|}
\hline 2 & 7 & 6 & 0 \\
\hline 2 & 9 & 22 & 0 \\
\hline 2 & 12 & 25 & 0 \\
\hline 2 & 7 & 17 & 0 \\
\hline 2 & 21 & 9 & 0 \\
\hline 2 & 22 & 9 & 0 \\
\hline 2 & 11 & 22 & 0 \\
\hline 2 & 13 & 4 & 0 \\
\hline 2 & 11 & 28 & 0 \\
\hline 2 & 11 & 20 & 0 \\
\hline 2 & 14 & 25 & 0 \\
\hline 2 & 4 & 13 & 0 \\
\hline 2 & 6 & 23 & 0 \\
\hline 2 & 9 & 17 & 0 \\
\hline 2 & 19 & 8 & 0 \\
\hline 2 & 15 & 27 & 0 \\
\hline 2 & 5 & 21 & 0 \\
\hline 2 & 5 & 8 & 0 \\
\hline 2 & 10 & 19 & 0 \\
\hline 2 & 4 & 20 & 0 \\
\hline 2 & 9 & 18 & 0 \\
\hline 2 & 9 & 7 & 0 \\
\hline 2 & 9 & 7 & 0 \\
\hline 2 & 9 & 17 & 0 \\
\hline 2 & 6 & 9 & 0 \\
\hline 2 & 5 & 7 & 0 \\
\hline 2 & 16 & 12 & 0 \\
\hline 2 & 14 & 19 & 0 \\
\hline 2 & 14 & 21 & 0 \\
\hline 2 & 8 & 9 & 0 \\
\hline 2 & 12 & 11 & 0 \\
\hline 2 & 16 & 5 & 0 \\
\hline 2 & 11 & 31 & 0 \\
\hline 2 & 20 & 13 & 0 \\
\hline 2 & 4 & 16 & 0 \\
\hline 2 & 10 & 4 & 0 \\
\hline 2 & 16 & 18 & 0 \\
\hline 2 & 6 & 10 & 0 \\
\hline 2 & 5 & 18 & 0 \\
\hline 2 & 5 & 12 & 0 \\
\hline 2 & 5 & 10 & 0 \\
\hline 2 & 20 & 12 & 0 \\
\hline 2 & 13 & 10 & 0 \\
\hline 2 & 5 & 14 & 0 \\
\hline 2 & 19 & 10 & 0 \\
\hline 2 & 24 & 4 & 0 \\
\hline 2 & 5 & 16 & 0 \\
\hline 2 & 11 & 12 & 0 \\
\hline 2 & 4 & 15 & 0 \\
\hline 2 & 13 & 26 & 0 \\
\hline 2 & 20 & 33 & 0 \\
\hline 2 & 8 & 7 & 0 \\
\hline 2 & 9 & 25 & 0 \\
\hline
\end{tabular}




\begin{tabular}{|c|c|}
\hline$x$ & 6833116 \\
\hline$x$ & 6833233 \\
\hline$x$ & 6834427 \\
\hline$x$ & 6834690 \\
\hline$x$ & 6837432 \\
\hline$x$ & 6837890 \\
\hline$x$ & 6838967 \\
\hline$x$ & 6840540 \\
\hline$x$ & 6841072 \\
\hline$x$ & 6841134 \\
\hline$x$ & 6841736 \\
\hline$x$ & 6852488 \\
\hline$X$ & 6853144 \\
\hline$x$ & 6853608 \\
\hline$x$ & 6853728 \\
\hline$x$ & 6863823 \\
\hline$x$ & 6863853 \\
\hline$x$ & 6864398 \\
\hline$x$ & 6864953 \\
\hline$x$ & 6865076 \\
\hline$x$ & 6865418 \\
\hline$x$ & 6865436 \\
\hline$x$ & 6865772 \\
\hline$x$ & 6865776 \\
\hline$x$ & 6866150 \\
\hline$x$ & 6866242 \\
\hline$x$ & 6866705 \\
\hline$x$ & 6866894 \\
\hline$x$ & 6867089 \\
\hline$x$ & 6867224 \\
\hline$x$ & 6867471 \\
\hline$x$ & 6867533 \\
\hline$x$ & 6872304 \\
\hline$x$ & 6873307 \\
\hline$x$ & 6873907 \\
\hline$x$ & 6873973 \\
\hline$x$ & 6874380 \\
\hline$x$ & 6874526 \\
\hline$x$ & 6874760 \\
\hline$x$ & 6874849 \\
\hline$x$ & 6874955 \\
\hline$x$ & 6875016 \\
\hline$x$ & 6877702 \\
\hline$x$ & 6883324 \\
\hline$x$ & 6884373 \\
\hline$x$ & 6887814 \\
\hline$x$ & 6888285 \\
\hline$x$ & 6888483 \\
\hline$x$ & 6888697 \\
\hline$x$ & 6890251 \\
\hline$X$ & 6890387 \\
\hline$x$ & 6890480 \\
\hline$x$ & 6890537 \\
\hline
\end{tabular}

$\begin{array}{rl}10 & 2 \\ 11 & \\ 11 & \\ 10 & \\ 11 & \\ 11 & 13 \\ 16 & 3 \\ 20 & 1 \\ 5 & 33 \\ 5 & 15 \\ 7 & \end{array}$

6

6

8

5

13

33

10

33

150

26

8

22

17

15

20

10

12

17

19

18

15

4

7

14

5

5

13

4

5

7

13

9

18

24

25

21

5

13

10

12 


\begin{tabular}{|c|c|}
\hline$x$ & 6891027 \\
\hline$x$ & 6893009 \\
\hline$x$ & 6893788 \\
\hline$x$ & 6893854 \\
\hline$x$ & 6893933 \\
\hline$x$ & 6894906 \\
\hline$x$ & 6895007 \\
\hline$x$ & 6895275 \\
\hline$x$ & 6895315 \\
\hline$x$ & 6899450 \\
\hline$x$ & 6902884 \\
\hline$x$ & 6903008 \\
\hline$x$ & 6903336 \\
\hline$x$ & 6903753 \\
\hline$x$ & 6903888 \\
\hline$x$ & 6903975 \\
\hline$x$ & 6904077 \\
\hline$x$ & 6904662 \\
\hline$x$ & 6905227 \\
\hline$x$ & 6905734 \\
\hline$x$ & 6905903 \\
\hline$x$ & 6906025 \\
\hline$x$ & 6906193 \\
\hline$x$ & 6910461 \\
\hline$x$ & 6915771 \\
\hline$x$ & 6925482 \\
\hline$x$ & 6927637 \\
\hline$x$ & 6927719 \\
\hline$x$ & 6928155 \\
\hline$x$ & 6929846 \\
\hline$x$ & 6930071 \\
\hline$x$ & 6931024 \\
\hline$x$ & 6931439 \\
\hline$x$ & 6933249 \\
\hline$x$ & 6933861 \\
\hline$x$ & 6934450 \\
\hline$x$ & 6934656 \\
\hline$x$ & 6935235 \\
\hline$x$ & 6935304 \\
\hline$x$ & 6938470 \\
\hline$x$ & 6938528 \\
\hline$x$ & 6938990 \\
\hline$x$ & 6940471 \\
\hline$x$ & 6941907 \\
\hline$x$ & 6942401 \\
\hline$x$ & 6943008 \\
\hline$x$ & 6943215 \\
\hline$x$ & 6944239 \\
\hline$x$ & 6945966 \\
\hline$x$ & 6947039 \\
\hline$x$ & 6947120 \\
\hline$x$ & 6947162 \\
\hline$x$ & 6947199 \\
\hline
\end{tabular}

\begin{tabular}{|c|c|c|}
\hline 13 & 5 & 0 \\
\hline 16 & 7 & 0 \\
\hline 25 & 24 & 0 \\
\hline 23 & 16 & 0 \\
\hline 20 & 9 & 0 \\
\hline 13 & 8 & 0 \\
\hline 18 & 13 & 0 \\
\hline 18 & 6 & 0 \\
\hline 15 & 4 & 0 \\
\hline 7 & 9 & 0 \\
\hline 5 & 11 & 0 \\
\hline 21 & 19 & 0 \\
\hline 10 & 23 & 0 \\
\hline 5 & 12 & 0 \\
\hline 8 & 21 & 0 \\
\hline 8 & 29 & 0 \\
\hline 4 & 19 & 0 \\
\hline 5 & 21 & 0 \\
\hline 5 & 7 & 0 \\
\hline 6 & 14 & 0 \\
\hline 14 & 20 & 0 \\
\hline 13 & 33 & 0 \\
\hline 5 & 16 & 0 \\
\hline 11 & 34 & 0 \\
\hline 7 & 18 & 0 \\
\hline 11 & 12 & 0 \\
\hline 19 & 13 & 0 \\
\hline 6 & 19 & 0 \\
\hline 9 & 5 & 0 \\
\hline 4 & 21 & 0 \\
\hline 12 & 8 & 0 \\
\hline 10 & 16 & 0 \\
\hline 11 & 14 & 0 \\
\hline 28 & 5 & 0 \\
\hline 16 & 9 & 0 \\
\hline 23 & 9 & 0 \\
\hline 28 & 14 & 0 \\
\hline 21 & 8 & 0 \\
\hline 8 & 7 & 0 \\
\hline 33 & 10 & 0 \\
\hline 27 & 7 & 0 \\
\hline 9 & 16 & 0 \\
\hline 17 & 6 & 0 \\
\hline 7 & 23 & 0 \\
\hline 11 & 10 & 0 \\
\hline 8 & 23 & 0 \\
\hline 9 & 18 & 0 \\
\hline 10 & 9 & 0 \\
\hline 8 & 24 & 0 \\
\hline 7 & 7 & 0 \\
\hline 18 & 6 & 0 \\
\hline 17 & 6 & 0 \\
\hline 6 & 7 & \\
\hline
\end{tabular}




$\begin{array}{ll}X & 6947207 \\ X & 6947615 \\ X & 6951380 \\ X & 6952118 \\ X & 6956161 \\ X & 6956979 \\ X & 6962311 \\ X & 6962367 \\ X & 6962395 \\ X & 6963192 \\ X & 6964189 \\ X & 6964611 \\ X & 6964671 \\ X & 6964698 \\ X & 6964975 \\ X & 6965050 \\ X & 6965368 \\ X & 6965601 \\ X & 6967871 \\ X & 6967880 \\ X & 6970406 \\ X & 6970571 \\ X & 6972670 \\ X & 6973869 \\ X & 6981350 \\ X & 6983310 \\ X & 6984954 \\ X & 6996014 \\ X & 6997713 \\ X & 7001469 \\ X & 7001513 \\ X & 7001714 \\ X & 7002175 \\ X & 7002298 \\ X & 7002352 \\ X & 7004221 \\ X & 7004304 \\ X & 7004932 \\ X & 7005159 \\ X & 7005476 \\ X & 7007598 \\ X & 7010343 \\ X & 7018239 \\ X & 7031999 \\ X & 705057679 \\ X & \\ X & 7055336 \\ X & 70590301 \\ X & \end{array}$

\section{2}

2

2

2

2

2

2

2

2

2

2

2

2

2

2

2

2

2

2

2

2

2

2

2

2

2

2

2

2

2

2

2

2

2

2

2

2

2

2

2

2

2

2

2

2

2

2

2

2

2

2

2

2
6

11

10

4

12

6

14

16

12

7

19

24

6

6

19

6

6

7
15

17

15

21

5

13

7

9

12

23

7

20

9

16

21

16

25

10

29

9

13

17

17

11

18

7

17

4

10

8

4

4

9

29

17
11

33

4

14

12

21

6

9

21

7

4

10

11

7

11

15

6

18

7

6

20

17

9

21

13

4

12

6

16

21

4

9

6

8

14

7

11

18

6

7

24

6

10

18

9

17

25

13

13

9

16

16

11
0

0

0

0

0

0

0

0

0

0

0

0

0

0

0

0

0

0

0

0

0

0

0

0

0

0

0

0

0

0

0

0

0

0

0

0

0

0

0

0

0

0

0

0

0

0

0

0

0 


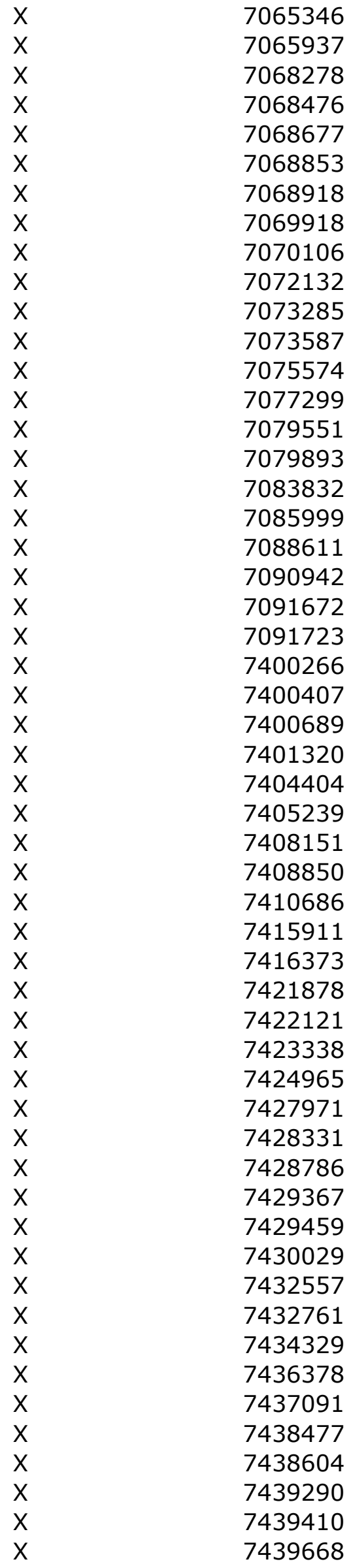

\begin{tabular}{|c|c|c|}
\hline \multirow{2}{*}{$\begin{array}{l}2 \\
2\end{array}$} & 6 & 18 \\
\hline & 8 & 12 \\
\hline 2 & 9 & 10 \\
\hline 2 & 6 & 12 \\
\hline 2 & 7 & 8 \\
\hline 2 & 19 & 15 \\
\hline 2 & 5 & 13 \\
\hline 2 & 15 & 15 \\
\hline 2 & 4 & 18 \\
\hline 2 & 11 & 21 \\
\hline 2 & 9 & 13 \\
\hline 2 & 4 & 10 \\
\hline 2 & 7 & 5 \\
\hline 2 & 4 & 11 \\
\hline 2 & 14 & 4 \\
\hline 2 & 35 & 4 \\
\hline 2 & 15 & 20 \\
\hline 2 & 17 & 4 \\
\hline 2 & 6 & 11 \\
\hline 2 & 12 & 14 \\
\hline 2 & 6 & 15 \\
\hline 2 & 7 & 16 \\
\hline 2 & 16 & 6 \\
\hline 2 & 6 & 6 \\
\hline 2 & 8 & 5 \\
\hline 2 & 22 & 15 \\
\hline 2 & 13 & 7 \\
\hline 2 & 17 & 7 \\
\hline 2 & 7 & 8 \\
\hline 2 & 7 & 6 \\
\hline 2 & 21 & 14 \\
\hline 2 & 6 & 7 \\
\hline 2 & 16 & 10 \\
\hline 2 & 23 & 5 \\
\hline 2 & 8 & 5 \\
\hline 2 & 13 & 4 \\
\hline 2 & 11 & 11 \\
\hline 2 & 4 & 11 \\
\hline 2 & 15 & 9 \\
\hline 2 & 16 & 11 \\
\hline 2 & 14 & 7 \\
\hline 2 & 21 & 16 \\
\hline 2 & 20 & 12 \\
\hline 2 & 21 & 8 \\
\hline 2 & 14 & 24 \\
\hline 2 & 24 & 11 \\
\hline 2 & 9 & 5 \\
\hline 2 & 13 & 13 \\
\hline 2 & 24 & 10 \\
\hline 2 & 9 & 4 \\
\hline 2 & 9 & 7 \\
\hline 2 & 10 & 6 \\
\hline 2 & 35 & 13 \\
\hline
\end{tabular}




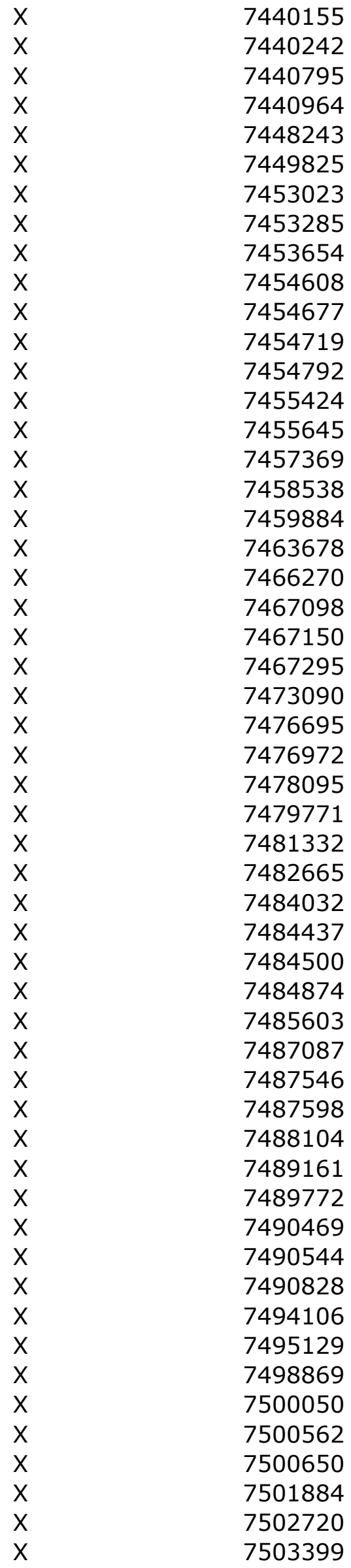

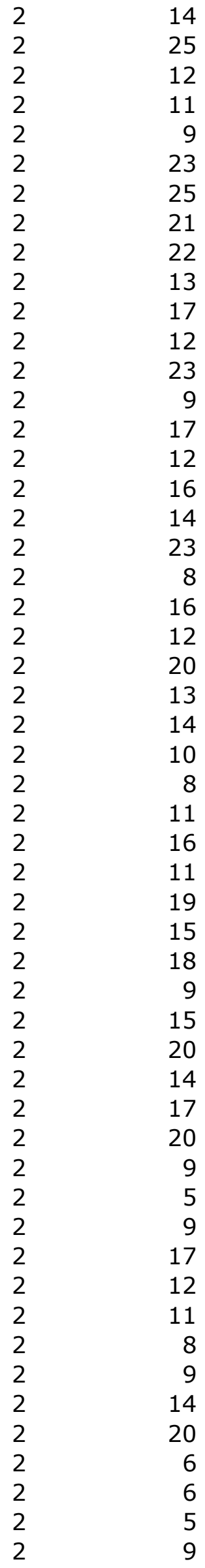

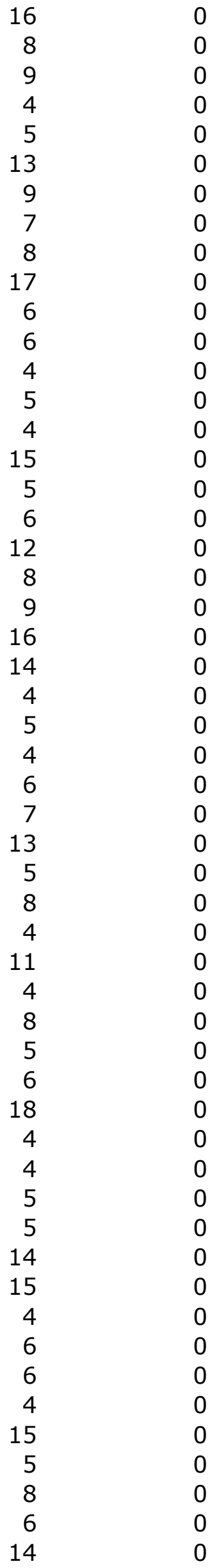




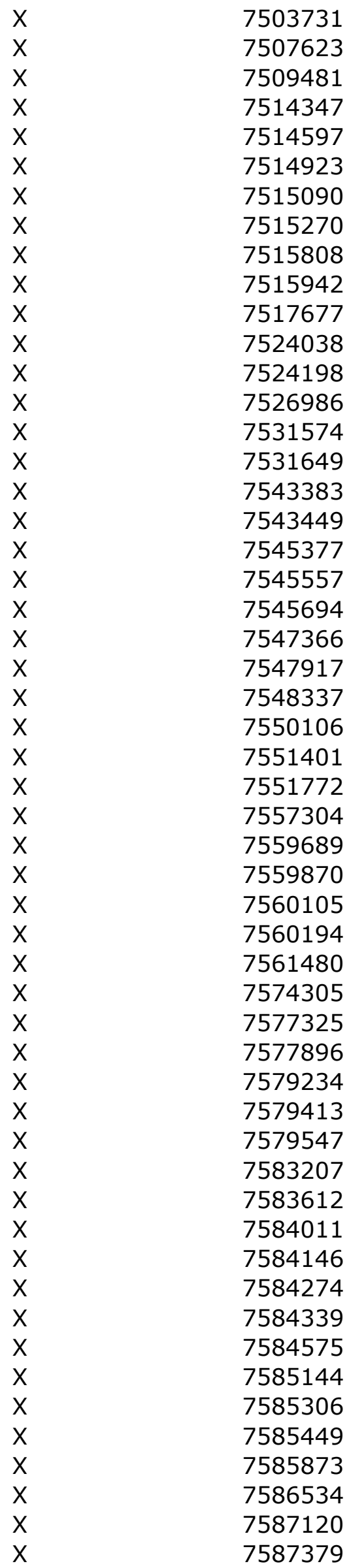

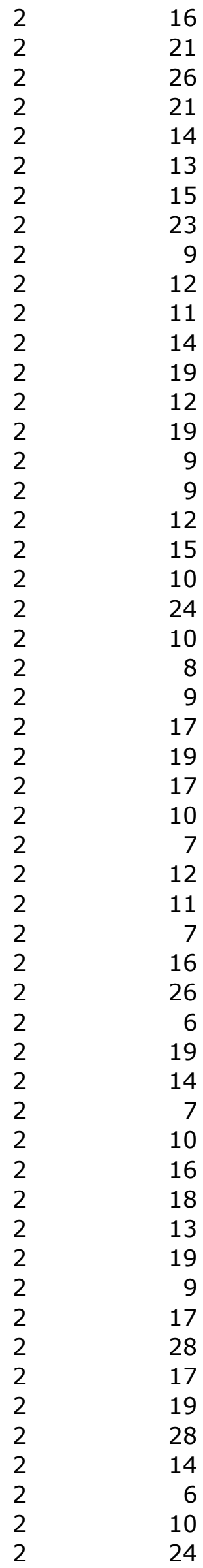

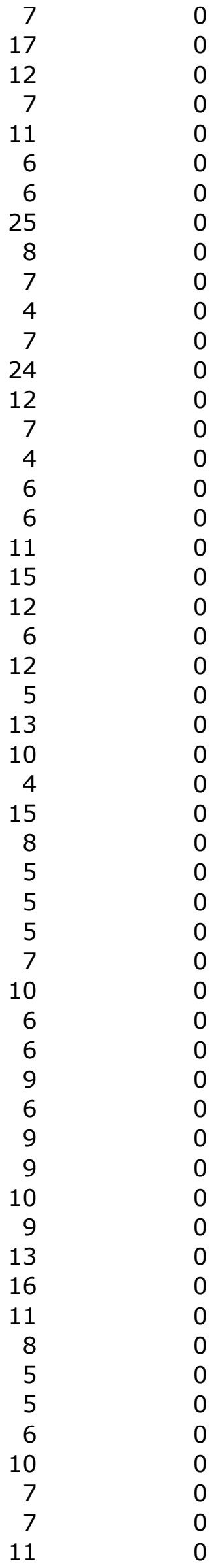




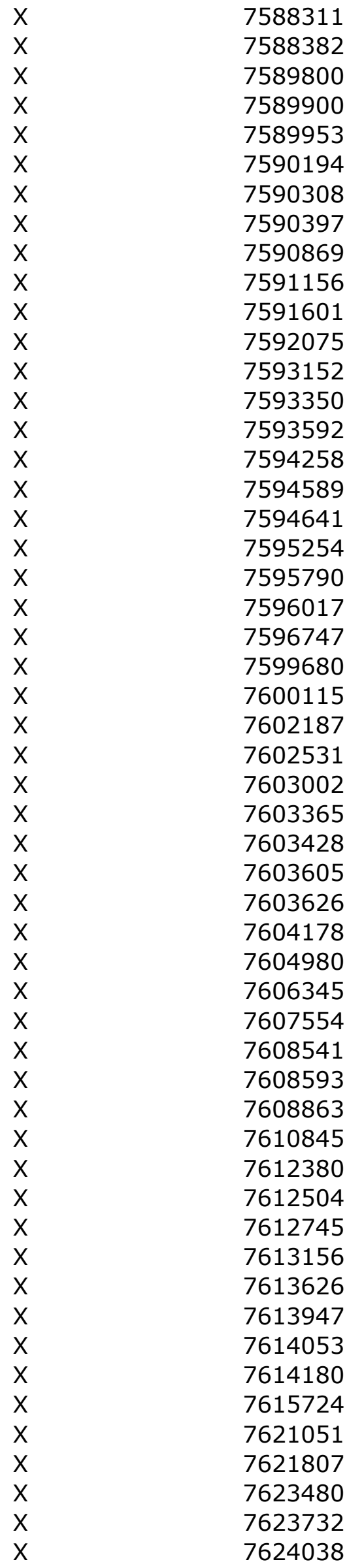

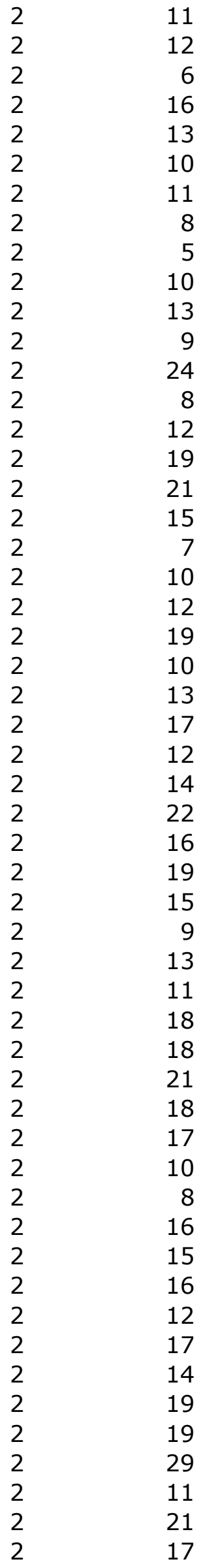

$$
\begin{array}{rl}
5 & 0 \\
10 & 0 \\
7 & 0 \\
12 & 0 \\
6 & 0 \\
6 & 0 \\
8 & 0 \\
5 & 0 \\
7 & 0 \\
11 & 0 \\
8 & 0 \\
6 & 0 \\
5 & 0 \\
4 & 0 \\
6 & 0
\end{array}
$$$$
10
$$$$
12
$$$$
6
$$$$
5
$$$$
11
$$$$
6
$$$$
4
$$$$
4
$$$$
11
$$$$
5
$$$$
7
$$$$
16
$$$$
16-0
$$$$
12
$$$$
8
$$$$
7
$$$$
10
$$$$
10
$$$$
16
$$$$
21
$$$$
4
$$$$
8
$$$$
9
$$$$
5
$$$$
8
$$$$
5
$$ 


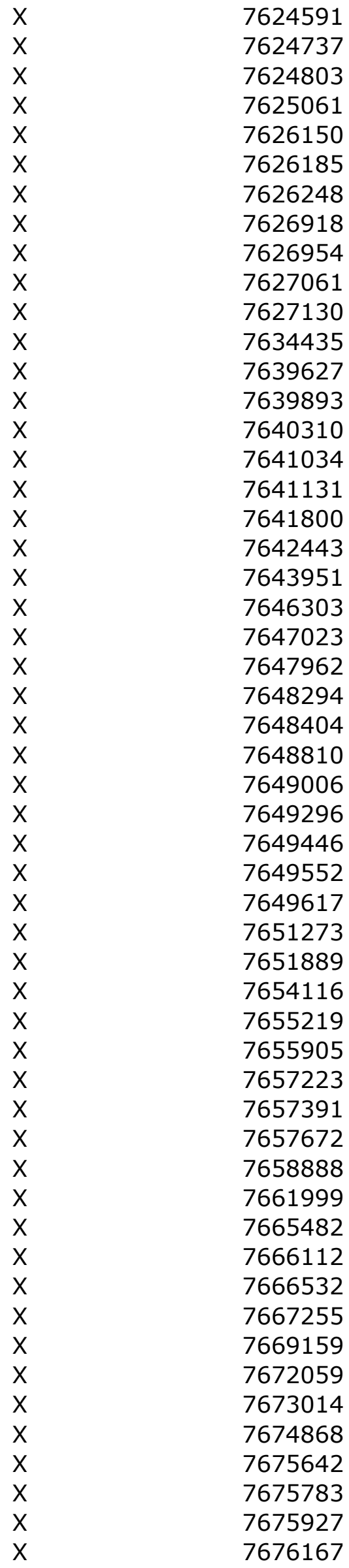

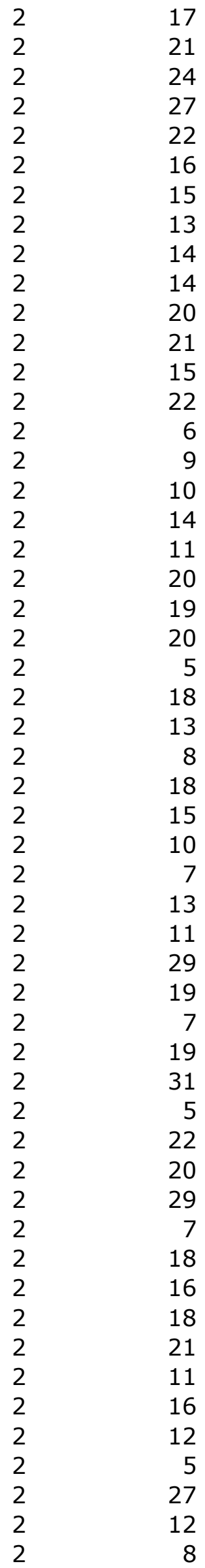

1

14

19

15

6

12
7

6

6

5

5

4

14

13

5

5

9

5

4

7

7

9

$7 \quad 0$

10

4

5

9

4

10

11

5

4

9

6

6

13

9

6

5

8

22

6

5

6

8

6

7

10

5

8

7

8

8

0

0

0

0

0

0

0

0

0

0

0

0

0

0

0

0

0

0

0

0

0

0

0

0

0

0

0

0

0

0

0

0

0

0

0

0

0

0

0

0

0 


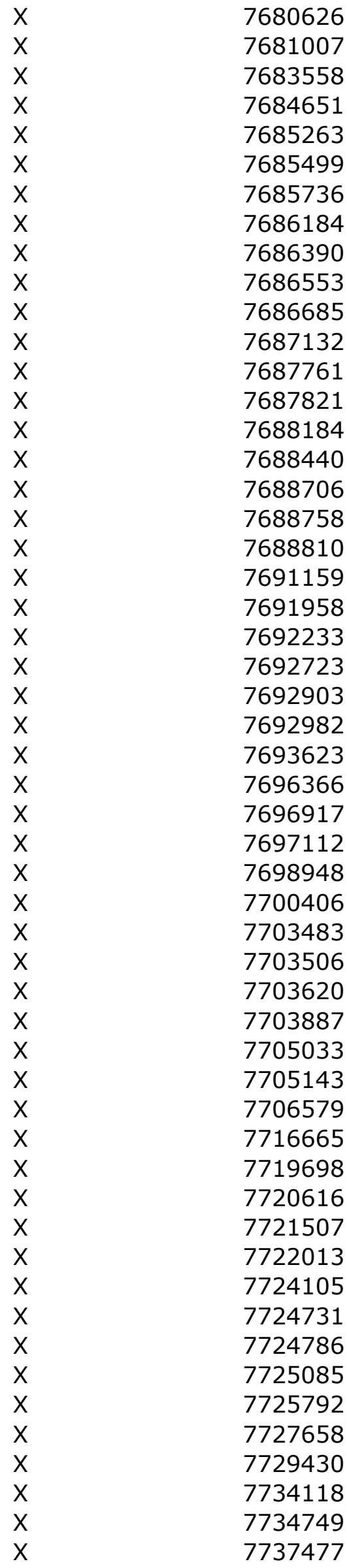

2
2
2
2
2
2
2
2
2
2
2
2
2
2
2
2
2
2
2
2
2
2
2
2
2
2
2
2
2
2
2
2
2
2
2
2
2
2
2
2
2
2
2
2
2
2
2
2
2
2
2

8
12
19
13
19
16
22
15

5

8

8

5

7

6

7

16

4

$$
4
$$$$
7 \quad 0
$$

8

14

23

11

7

10

9

8

24

12

17

9

21

11

7

11

10

5

12

7

22

17

5

20

11

9

12

19

12

16

14

9

7

5

14

12

9

10

15

5

4

11
13

7

8

7

11

7
12

12

9

12

6

10

12

8

16

8

5

4

7

7

6

5

5

4

4

5

9

6

6

5

8

12

6

7

6

6

5

9

5

14

21
0

0

0

0

0

0

0

0

0

0

0

0

0

0

0

0

0

0

0

0

0

0

0

0

0

0

0

0

0

0

0

0

0

0

0

0

0

0

0

0

0

0

0

0

0

0

0

0 


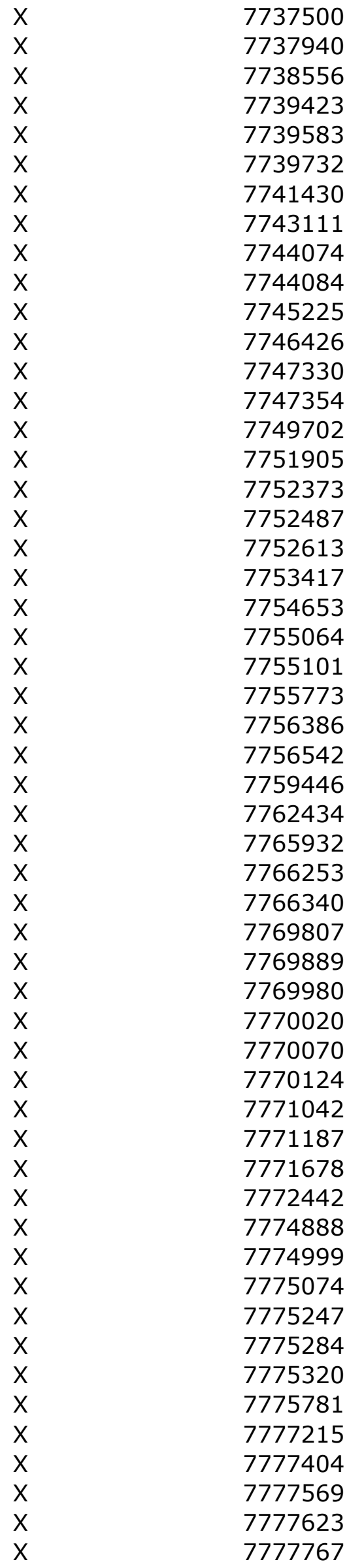

\begin{tabular}{|c|c|c|}
\hline \multirow{2}{*}{$\begin{array}{l}2 \\
2\end{array}$} & 15 & 19 \\
\hline & 19 & 11 \\
\hline 2 & 15 & 5 \\
\hline 2 & 14 & 6 \\
\hline 2 & 20 & 10 \\
\hline 2 & 20 & 17 \\
\hline 2 & 14 & 7 \\
\hline 2 & 4 & 22 \\
\hline 2 & 8 & 10 \\
\hline 2 & 10 & 9 \\
\hline 2 & 20 & 10 \\
\hline 2 & 21 & 15 \\
\hline 2 & 4 & 21 \\
\hline 2 & 5 & 29 \\
\hline 2 & 16 & 10 \\
\hline 2 & 9 & 25 \\
\hline 2 & 11 & 18 \\
\hline 2 & 12 & 15 \\
\hline 2 & 18 & 18 \\
\hline 2 & 6 & 8 \\
\hline 2 & 10 & 18 \\
\hline 2 & 10 & 15 \\
\hline 2 & 7 & 27 \\
\hline 2 & 4 & 20 \\
\hline 2 & 4 & 13 \\
\hline 2 & 5 & 10 \\
\hline 2 & 27 & 30 \\
\hline 2 & 5 & 18 \\
\hline 2 & 17 & 21 \\
\hline 2 & 14 & 4 \\
\hline 2 & 27 & 5 \\
\hline 2 & 17 & 5 \\
\hline 2 & 23 & 25 \\
\hline 2 & 7 & 13 \\
\hline 2 & 20 & 4 \\
\hline 2 & 13 & 6 \\
\hline 2 & 21 & 14 \\
\hline 2 & 39 & 5 \\
\hline 2 & 20 & 13 \\
\hline 2 & 6 & 15 \\
\hline 2 & 12 & 5 \\
\hline 2 & 9 & 7 \\
\hline 2 & 10 & 21 \\
\hline 2 & 7 & 33 \\
\hline 2 & 4 & 23 \\
\hline 2 & 3 & 35 \\
\hline 2 & 8 & 28 \\
\hline 2 & 15 & 24 \\
\hline 2 & 6 & 15 \\
\hline 2 & 9 & 17 \\
\hline 2 & 5 & 37 \\
\hline 2 & 15 & 24 \\
\hline 2 & 6 & 13 \\
\hline
\end{tabular}




\begin{tabular}{|c|c|}
\hline$x$ & 7778210 \\
\hline$x$ & 7778366 \\
\hline$X$ & 7778407 \\
\hline$x$ & 7778650 \\
\hline$x$ & 7780412 \\
\hline$x$ & 7786718 \\
\hline$x$ & 7787359 \\
\hline$x$ & 7800341 \\
\hline$x$ & 7804678 \\
\hline$x$ & 7804852 \\
\hline$x$ & 7805494 \\
\hline$x$ & 7805622 \\
\hline$x$ & 7805664 \\
\hline$x$ & 7807628 \\
\hline$X$ & 7807774 \\
\hline$x$ & 7808026 \\
\hline$x$ & 7812158 \\
\hline$x$ & 7812374 \\
\hline$x$ & 7812726 \\
\hline$x$ & 7812923 \\
\hline$x$ & 7812964 \\
\hline$x$ & 7813471 \\
\hline$x$ & 7815292 \\
\hline$x$ & 7815898 \\
\hline$x$ & 7815973 \\
\hline$x$ & 7816391 \\
\hline$x$ & 7818730 \\
\hline$x$ & 7819371 \\
\hline$x$ & 7819430 \\
\hline$x$ & 7820811 \\
\hline$x$ & 7830565 \\
\hline$x$ & 7852355 \\
\hline$x$ & 7855784 \\
\hline$x$ & 7857438 \\
\hline$x$ & 7857597 \\
\hline$x$ & 7859504 \\
\hline$x$ & 7859566 \\
\hline$x$ & 7859843 \\
\hline$x$ & 7859871 \\
\hline$x$ & 7863423 \\
\hline$x$ & 7867378 \\
\hline$x$ & 7867418 \\
\hline$x$ & 7870516 \\
\hline$x$ & 7872270 \\
\hline$x$ & 7873112 \\
\hline$x$ & 7873291 \\
\hline$x$ & 7874197 \\
\hline$x$ & 7874278 \\
\hline$x$ & 7875816 \\
\hline$x$ & 7876090 \\
\hline$x$ & 7876387 \\
\hline$x$ & 7876420 \\
\hline$x$ & $78766 \mathrm{~S}$ \\
\hline
\end{tabular}

\begin{tabular}{|c|c|c|c|}
\hline 2 & 4 & 13 & 0 \\
\hline 2 & 12 & 4 & 0 \\
\hline 2 & 21 & 12 & 0 \\
\hline 2 & 5 & 9 & 0 \\
\hline 2 & 13 & 21 & 0 \\
\hline 2 & 6 & 12 & 0 \\
\hline 2 & 11 & 24 & 0 \\
\hline 2 & 19 & 13 & 0 \\
\hline 2 & 12 & 4 & 0 \\
\hline 2 & 26 & 6 & 0 \\
\hline 2 & 20 & 5 & 0 \\
\hline 2 & 20 & 7 & 0 \\
\hline 2 & 14 & 5 & 0 \\
\hline 2 & 4 & 14 & 0 \\
\hline 2 & 4 & 14 & 0 \\
\hline 2 & 13 & 9 & 0 \\
\hline 2 & 18 & 11 & 0 \\
\hline 2 & 17 & 19 & 0 \\
\hline 2 & 10 & 8 & 0 \\
\hline 2 & 12 & 22 & 0 \\
\hline 2 & 11 & 25 & 0 \\
\hline 2 & 9 & 25 & 0 \\
\hline 2 & 20 & 10 & 0 \\
\hline 2 & 11 & 11 & 0 \\
\hline 2 & 6 & 21 & 0 \\
\hline 2 & 13 & 5 & 0 \\
\hline 2 & 15 & 11 & 0 \\
\hline 2 & 11 & 8 & 0 \\
\hline 2 & 12 & 14 & 0 \\
\hline 2 & 19 & 15 & 0 \\
\hline 2 & 9 & 6 & 0 \\
\hline 2 & 8 & 6 & 0 \\
\hline 2 & 10 & 8 & 0 \\
\hline 2 & 19 & 30 & 0 \\
\hline 2 & 8 & 17 & 0 \\
\hline 2 & 4 & 32 & 0 \\
\hline 2 & 6 & 18 & 0 \\
\hline 2 & 6 & 7 & 0 \\
\hline 2 & 15 & 14 & 0 \\
\hline 2 & 13 & 6 & 0 \\
\hline 2 & 32 & 15 & 0 \\
\hline 2 & 15 & 13 & 0 \\
\hline 2 & 9 & 9 & 0 \\
\hline 2 & 14 & 11 & 0 \\
\hline 2 & 4 & 23 & 0 \\
\hline 2 & 18 & 19 & 0 \\
\hline 2 & 5 & 19 & 0 \\
\hline 2 & 7 & 9 & 0 \\
\hline 2 & 12 & 21 & 0 \\
\hline 2 & 12 & 11 & 0 \\
\hline 2 & 21 & 7 & 0 \\
\hline 2 & 18 & 4 & 0 \\
\hline 2 & 17 & 5 & 0 \\
\hline
\end{tabular}




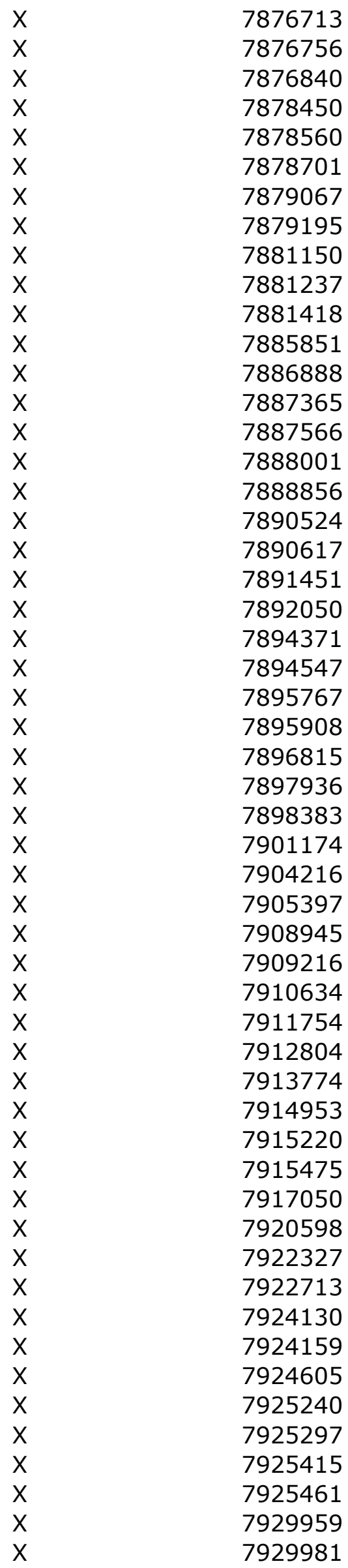

\begin{tabular}{|c|c|c|}
\hline \multirow{2}{*}{$\begin{array}{l}2 \\
2\end{array}$} & 21 & 8 \\
\hline & 20 & 4 \\
\hline 2 & 15 & 4 \\
\hline 2 & 6 & 26 \\
\hline 2 & 4 & 11 \\
\hline 2 & 6 & 12 \\
\hline 2 & 11 & 26 \\
\hline 2 & 8 & 21 \\
\hline 2 & 6 & 18 \\
\hline 2 & 11 & 13 \\
\hline 2 & 4 & 11 \\
\hline 2 & 4 & 18 \\
\hline 2 & 10 & 15 \\
\hline 2 & 11 & 10 \\
\hline 2 & 10 & 6 \\
\hline 2 & 12 & 5 \\
\hline 2 & 11 & 8 \\
\hline 2 & 5 & 13 \\
\hline 2 & 7 & 16 \\
\hline 2 & 4 & 17 \\
\hline 2 & 21 & 6 \\
\hline 2 & 11 & 11 \\
\hline 2 & 27 & 18 \\
\hline 2 & 18 & 5 \\
\hline 2 & 27 & 15 \\
\hline 2 & 15 & 7 \\
\hline 2 & 7 & 19 \\
\hline 2 & 5 & 7 \\
\hline 2 & 12 & 16 \\
\hline 2 & 12 & 6 \\
\hline 2 & 14 & 5 \\
\hline 2 & 5 & 19 \\
\hline 2 & 16 & 12 \\
\hline 2 & 5 & 11 \\
\hline 2 & 5 & 6 \\
\hline 2 & 23 & 10 \\
\hline 2 & 18 & 6 \\
\hline 2 & 15 & 5 \\
\hline 2 & 26 & 7 \\
\hline 2 & 14 & 14 \\
\hline 2 & 5 & 7 \\
\hline 2 & 13 & 15 \\
\hline 2 & 15 & 7 \\
\hline 2 & 11 & 10 \\
\hline 2 & 10 & 8 \\
\hline 2 & 8 & 5 \\
\hline 2 & 13 & 4 \\
\hline 2 & 19 & 4 \\
\hline 2 & 22 & 7 \\
\hline 2 & 5 & 12 \\
\hline 2 & 26 & 17 \\
\hline 2 & 23 & 5 \\
\hline 2 & 31 & 8 \\
\hline
\end{tabular}




\begin{tabular}{|c|c|}
\hline$x$ & 7930415 \\
\hline$x$ & 7930582 \\
\hline$X$ & 7931406 \\
\hline$x$ & 7932524 \\
\hline$x$ & 7933157 \\
\hline$x$ & 7935090 \\
\hline$x$ & 7936574 \\
\hline$x$ & 7936931 \\
\hline$x$ & 7937243 \\
\hline$x$ & 7937261 \\
\hline$x$ & 7937787 \\
\hline$x$ & 7938088 \\
\hline$x$ & 7938159 \\
\hline$x$ & 7938320 \\
\hline$x$ & 7938544 \\
\hline$x$ & 7940801 \\
\hline$x$ & 7941348 \\
\hline$x$ & 7941967 \\
\hline$x$ & 7943668 \\
\hline$x$ & 7944331 \\
\hline$x$ & 7947508 \\
\hline$x$ & 7948801 \\
\hline$x$ & 7950498 \\
\hline$x$ & 7951007 \\
\hline$x$ & 7951804 \\
\hline$x$ & 7952006 \\
\hline$x$ & 7952110 \\
\hline$x$ & 7952663 \\
\hline$x$ & 7952946 \\
\hline$x$ & 7953814 \\
\hline$x$ & 7955794 \\
\hline$x$ & 7956868 \\
\hline$x$ & 7957009 \\
\hline$x$ & 7957446 \\
\hline$x$ & 7957907 \\
\hline$x$ & 7958107 \\
\hline$x$ & 7958544 \\
\hline$x$ & 7958651 \\
\hline$x$ & 7960570 \\
\hline$x$ & 7960909 \\
\hline$x$ & 7961358 \\
\hline$x$ & 7962654 \\
\hline$x$ & 7963154 \\
\hline$x$ & 7964852 \\
\hline$x$ & 7965345 \\
\hline$x$ & 7966162 \\
\hline$x$ & 7966500 \\
\hline$x$ & 7966972 \\
\hline$x$ & 7967170 \\
\hline$x$ & 7967428 \\
\hline$x$ & 7967713 \\
\hline$x$ & 7967740 \\
\hline$x$ & 796803 \\
\hline
\end{tabular}

2

2

2

2

2

2

2

2

2

2

2

2

2

2

2

2

2

2

2

2

2

2

2

2

2

2

2

2

2

2

2

2

2

2

2

2

2

2

2

2

2

2

2

2

2

2

2

2

2

2

2

2

2
30

36

23

35

11

8

17

20

6

5

10

12

7

4

13

8

10

23

9

6

5

6

12

5

17

10

13

5

7

6

4

7

11

4

11

6

14

18

5

8

4

11

14

24

11

24

19

16

10

11

22

11

13

\section{6}

5

5

6

17

28

15

7

5

13

30

23

6

20

9

9

13

18

23

20

5

28

15

19

17

23

7

23

13

19

13

15

12

28

10

27

28

12

30

27

8

29

10

8

16

11

9

19

12

11

17

8
0

0

0

0

0

0

0

0

0

0

0

0

0

0

0

0

0

0

0

0

0

0

0

0

0

0

0

0

0

0

0

0

0

0

0

0

0

0

0

0

0

0

0

0

0

0

0

0

0

0 


\begin{tabular}{|c|c|}
\hline$x$ & 7968154 \\
\hline$x$ & 7969323 \\
\hline$X$ & 7969756 \\
\hline$x$ & 7970291 \\
\hline$x$ & 7970367 \\
\hline$x$ & 7970522 \\
\hline$x$ & 7970888 \\
\hline$x$ & 7971686 \\
\hline$x$ & 7971722 \\
\hline$x$ & 7971839 \\
\hline$x$ & 7971845 \\
\hline$x$ & 7972058 \\
\hline$x$ & 7972250 \\
\hline$x$ & 7972854 \\
\hline$X$ & 7972973 \\
\hline$x$ & 7973443 \\
\hline$x$ & 7973673 \\
\hline$x$ & 7973904 \\
\hline$x$ & 7974232 \\
\hline$x$ & 7974488 \\
\hline$x$ & 7974749 \\
\hline$x$ & 7975209 \\
\hline$x$ & 7977084 \\
\hline$x$ & 7977647 \\
\hline$x$ & 7977960 \\
\hline$x$ & 7978612 \\
\hline$x$ & 7978768 \\
\hline$x$ & 7978956 \\
\hline$x$ & 7979346 \\
\hline$x$ & 7979862 \\
\hline$x$ & 7979887 \\
\hline$x$ & 7980126 \\
\hline$x$ & 7980308 \\
\hline$x$ & 7981871 \\
\hline$x$ & 7982289 \\
\hline$x$ & 7982688 \\
\hline$x$ & 7984886 \\
\hline$x$ & 7985380 \\
\hline$x$ & 7985577 \\
\hline$x$ & 7986298 \\
\hline$x$ & 7987515 \\
\hline$x$ & 7988361 \\
\hline$x$ & 7988960 \\
\hline$x$ & 7990561 \\
\hline$x$ & 8036209 \\
\hline$x$ & 8487714 \\
\hline$x$ & 8491505 \\
\hline$x$ & 8493370 \\
\hline$x$ & 8499315 \\
\hline$x$ & 8501683 \\
\hline$x$ & 8504648 \\
\hline$x$ & 8505465 \\
\hline$x$ & 85101 \\
\hline
\end{tabular}

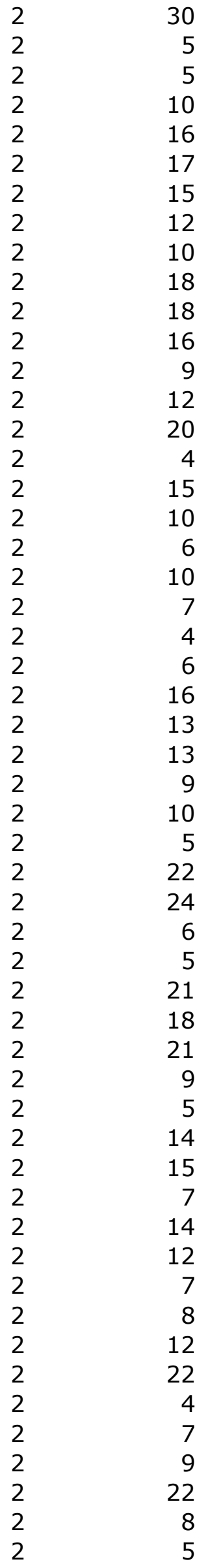

15

23

9

11

8

35

7

12

17

12

11

4

12

21

7

30

23

13

12

14

24

18

13

18

19

4

9

27

12

6

5

6

12

15

7

8

11

17

25

6

11

4

9

7

17

6

7

11

14

13

5

10

19
0

0

0

0

0

0

0

0

0

0

0

0

0

0

0

0

0

0

0

0

0

0

0

0

0

0

0

0

0

0

0

0

0

0

0

0

0

0

0

0

0

0

0

0

0

0
0

0

0

0 


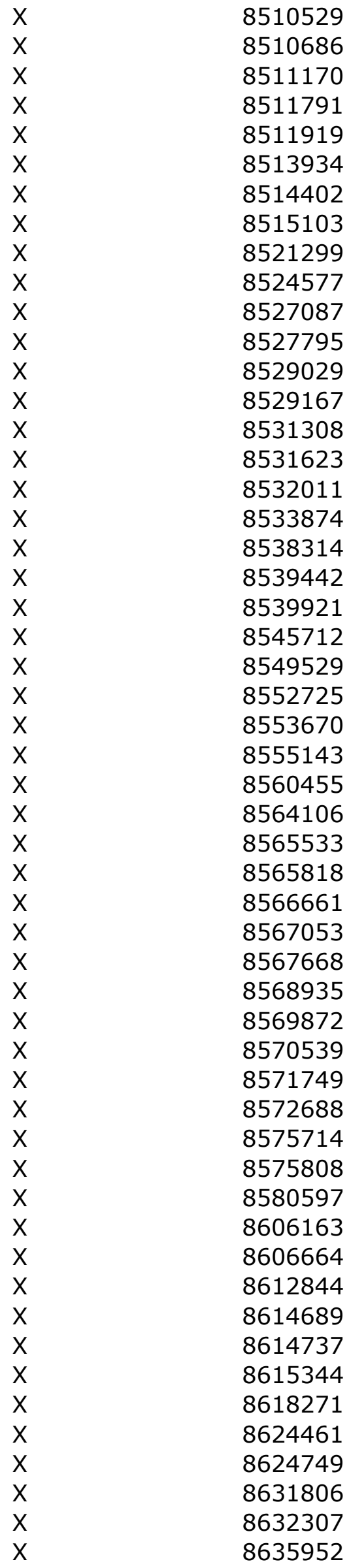

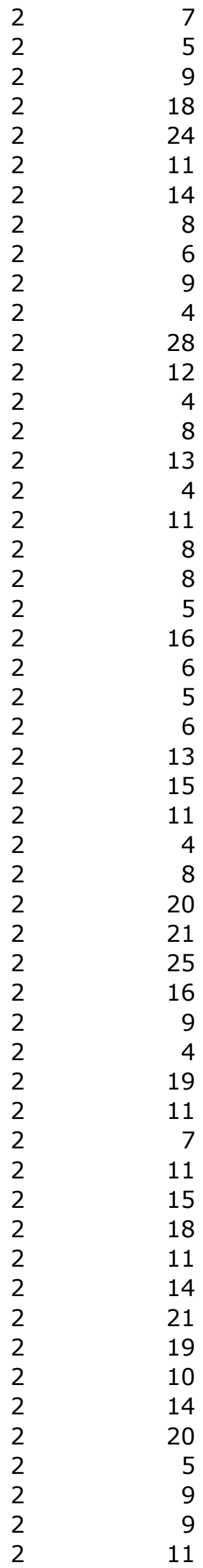

$\begin{array}{rl}14 & 0 \\ 23 & 0 \\ 18 & 0 \\ 7 & 0 \\ 7 & 0 \\ 6 & 0 \\ 13 & 0 \\ 6 & 0 \\ 15 & 0 \\ 20 & 0 \\ 17 & 0 \\ 7 & 0 \\ 17 & 0 \\ 15 & 0 \\ 10 & 0 \\ 5 & 0 \\ 23 & 0 \\ 6 & 0 \\ 22 & 0 \\ 19 & 0 \\ 10 & 0 \\ 8 & 0 \\ 17 & 0 \\ 5 & 0 \\ 25 & 0 \\ 5 & 0 \\ 4 & 0 \\ 13 & 0 \\ 20 & 0 \\ 6 & 0 \\ 5 & 0 \\ 11 & 0 \\ 11 & 0 \\ 6 & 0 \\ 7 & 0 \\ 13 & 0 \\ 5 & 0 \\ 11 & 0 \\ 5 & 0 \\ 5 & 0 \\ 5 & 0 \\ 5 & 0 \\ 5 & 0 \\ 5 & 0 \\ 5 & 0 \\ 5 & 0 \\ 5 & 0 \\ 5 & 0 \\ 5 & 0 \\ 5 & 0 \\ 5 & 0 \\ 5 & 0 \\ 5 & 0 \\ 5 & 0 \\ 12 & 0\end{array}$




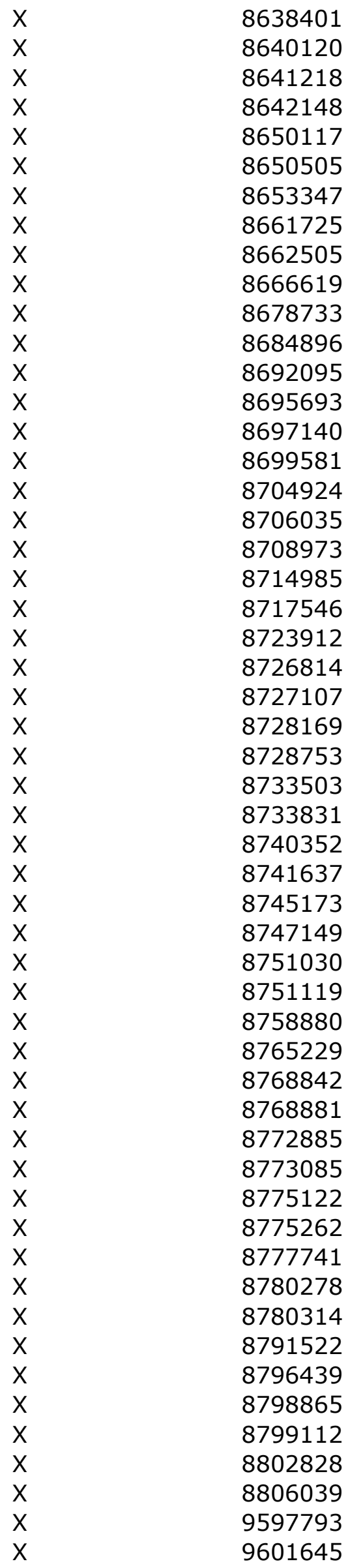

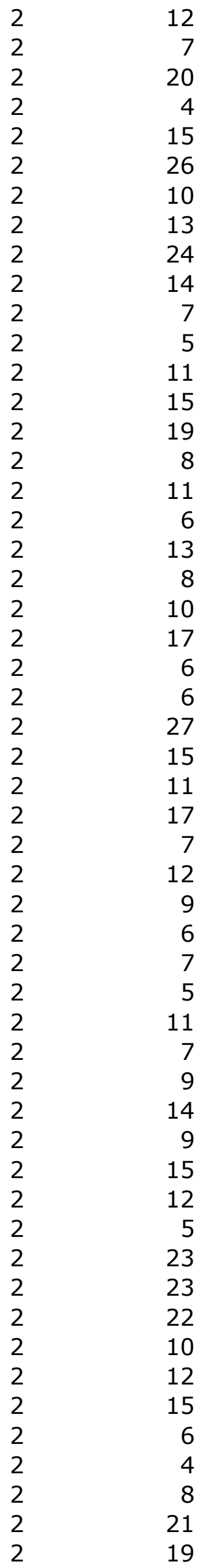

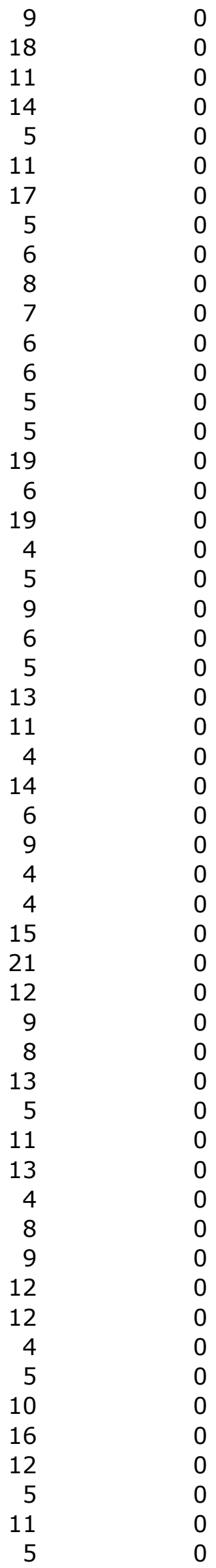




\begin{tabular}{|c|c|c|c|c|c|}
\hline$x$ & 9602106 & 2 & 30 & 4 & 0 \\
\hline$x$ & 9602734 & 2 & 20 & 6 & 0 \\
\hline$x$ & 9603557 & 2 & 20 & 10 & 0 \\
\hline$x$ & 9611528 & 2 & 11 & 7 & 0 \\
\hline$x$ & 9615995 & 2 & 5 & 9 & 0 \\
\hline$x$ & 9618231 & 2 & 16 & 7 & 0 \\
\hline$x$ & 9625657 & 2 & 22 & 14 & 0 \\
\hline$x$ & 9627309 & 2 & 10 & 12 & 0 \\
\hline$x$ & 9627839 & 2 & 10 & 11 & 0 \\
\hline$x$ & 9628907 & 2 & 13 & 12 & 0 \\
\hline$x$ & 9629335 & 2 & 23 & 6 & 0 \\
\hline$x$ & 9629956 & 2 & 6 & 12 & 0 \\
\hline$x$ & 9630867 & 2 & 14 & 10 & 0 \\
\hline$x$ & 9630948 & 2 & 6 & 5 & 0 \\
\hline$x$ & 9631597 & 2 & 19 & 13 & 0 \\
\hline$x$ & 9634021 & 2 & 10 & 4 & 0 \\
\hline$x$ & 9636020 & 2 & 4 & 30 & 0 \\
\hline$x$ & 9641019 & 2 & 4 & 13 & 0 \\
\hline$x$ & 9641705 & 2 & 21 & 10 & 0 \\
\hline$x$ & 9651206 & 2 & 7 & 5 & 0 \\
\hline$x$ & 9651269 & 2 & 10 & 6 & 0 \\
\hline$x$ & 9652605 & 2 & 23 & 9 & 0 \\
\hline$x$ & 9654455 & 2 & 19 & 15 & 0 \\
\hline$x$ & 9656532 & 2 & 4 & 13 & 0 \\
\hline$x$ & 9658615 & 2 & 5 & 7 & 0 \\
\hline$X$ & 9662485 & 2 & 11 & 9 & 0 \\
\hline$x$ & 9667968 & 2 & 4 & 23 & 0 \\
\hline$x$ & 9670601 & 2 & 6 & 17 & 0 \\
\hline$x$ & 9675170 & 2 & 10 & 9 & 0 \\
\hline$x$ & 9675467 & 2 & 9 & 17 & 0 \\
\hline$x$ & 9675515 & 2 & 7 & 14 & 0 \\
\hline$x$ & 9676028 & 2 & 22 & 6 & 0 \\
\hline$x$ & 9677176 & 2 & 15 & 6 & 0 \\
\hline$x$ & 9677446 & 2 & 18 & 5 & 0 \\
\hline$x$ & 9677562 & 2 & 11 & 4 & 0 \\
\hline$x$ & 9679038 & 2 & 4 & 11 & 0 \\
\hline$x$ & 9682766 & 2 & 13 & 10 & 0 \\
\hline$X$ & 9683334 & 2 & 13 & 24 & 0 \\
\hline$x$ & 9683395 & 2 & 12 & 5 & 0 \\
\hline$x$ & 9688125 & 2 & 13 & 15 & 0 \\
\hline$x$ & 9688497 & 2 & 7 & 5 & 0 \\
\hline$x$ & 9689106 & 2 & 8 & 8 & 0 \\
\hline$x$ & 9692076 & 2 & 11 & 10 & 0 \\
\hline$x$ & 9700477 & 2 & 18 & 8 & 0 \\
\hline$x$ & 9702571 & 2 & 12 & 4 & 0 \\
\hline$x$ & 9702811 & 2 & 11 & 4 & 0 \\
\hline$x$ & 9714680 & 2 & 11 & 14 & 0 \\
\hline$x$ & 9717786 & 2 & 4 & 17 & 0 \\
\hline$x$ & 9721863 & 2 & 10 & 19 & 0 \\
\hline$x$ & 9727544 & 2 & 4 & 12 & 0 \\
\hline$x$ & 9728233 & 2 & 10 & 7 & 0 \\
\hline$X$ & 9728514 & 2 & 10 & 7 & 0 \\
\hline$x$ & 9729305 & 2 & 4 & 15 & 0 \\
\hline
\end{tabular}




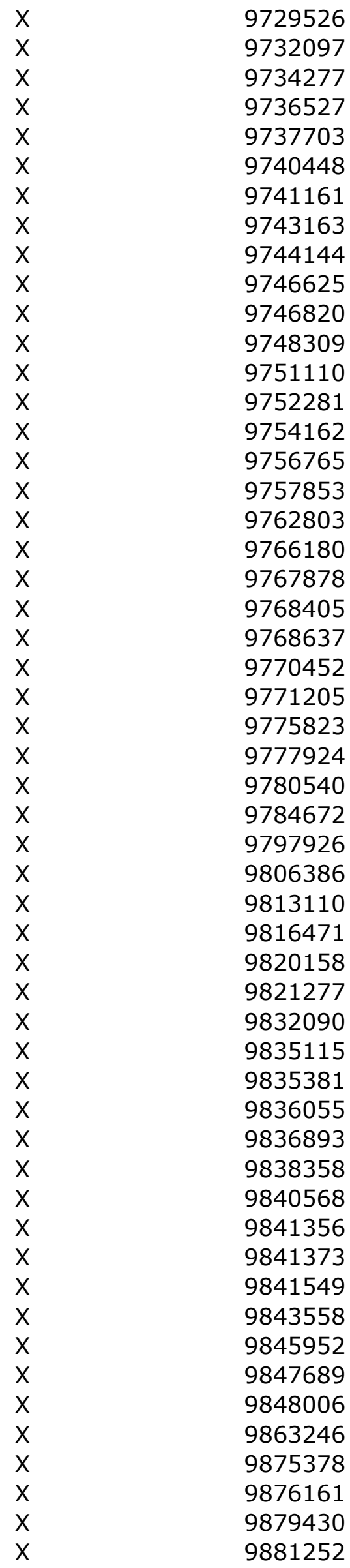

2

2

2

2

2

2

2

2

2

2

2

2

2

2

2

2

2

2

2

2

2

2

2

2

2

2

2

2

2

2

2

2

2

2

2

2

2

2

2

2

2

2

2

2

2

2

2

2

2

2

2

2

2

$\begin{array}{rr}24 & \\ 43 & \\ 9 & 14 \\ 13 & 13 \\ 5 & \\ 5 & 26 \\ 12 & 18 \\ 20 & 14 \\ 4 & 12\end{array}$

13

16

12

10

7

13

10

8

6

10

17

15

23

5

22

5

18

5

10

8

20

8

4

10

10

16

5

16

8

10

10

11

5

17

17

4

9

18

5

9

9

4

6
$4 \quad 0$

$5 \quad 0$

140

$13 \quad 0$

$5 \quad 0$

$26 \quad 0$

$18 \quad 0$

$14 \quad 0$

$12 \quad 0$

$7 \quad 0$

$8 \quad 0$

$14 \quad 0$

$7 \quad 0$

120

$19 \quad 0$

$\begin{array}{ll}5 & 0 \\ 10 & 0\end{array}$

$\begin{array}{ll}10 & 0\end{array}$

$7 \quad 0$

$11 \quad 0$

40

$17 \quad 0$

50

15

150

$10 \quad 0$

$8 \quad 0$

8

22

10

18

12

7

18

9

11

12

17

13

6

20

9

12

17

18

26

11

8

6

9

14
0

0

0

0

0

0

0

0

0

0

0

0

0

0

0

0

0

0

0

0

0

0 


\begin{tabular}{|c|c|}
\hline$x$ & 9888438 \\
\hline$x$ & 9889544 \\
\hline$x$ & 9905272 \\
\hline$x$ & 9905765 \\
\hline$x$ & 9908579 \\
\hline$x$ & 9909480 \\
\hline$x$ & 9911480 \\
\hline$x$ & 9916899 \\
\hline$x$ & 9926594 \\
\hline$x$ & 9926813 \\
\hline$x$ & 9987792 \\
\hline$x$ & 9988438 \\
\hline$x$ & 9998394 \\
\hline$x$ & 10000981 \\
\hline$x$ & 10005853 \\
\hline$x$ & 10015874 \\
\hline$x$ & 10023477 \\
\hline$x$ & 10027816 \\
\hline$x$ & 10030683 \\
\hline$x$ & 10041037 \\
\hline$x$ & 10058842 \\
\hline$x$ & 10063093 \\
\hline$x$ & 10065610 \\
\hline$x$ & 10069501 \\
\hline$x$ & 10070897 \\
\hline$x$ & 10071573 \\
\hline$x$ & 10072613 \\
\hline$x$ & 10074680 \\
\hline$x$ & 10082052 \\
\hline$x$ & 10082372 \\
\hline$x$ & 10083535 \\
\hline$x$ & 10089806 \\
\hline$x$ & 10111164 \\
\hline$x$ & 10112075 \\
\hline$x$ & 10117604 \\
\hline$x$ & 10122673 \\
\hline$x$ & 10132705 \\
\hline$x$ & 10141137 \\
\hline$x$ & 10147020 \\
\hline$x$ & 10152293 \\
\hline$x$ & 10152296 \\
\hline$x$ & 10181586 \\
\hline$x$ & 10204994 \\
\hline$x$ & 10236036 \\
\hline$x$ & 10236807 \\
\hline$X$ & 10238306 \\
\hline$x$ & 10265108 \\
\hline$x$ & 10278075 \\
\hline$x$ & 10278665 \\
\hline$x$ & 10286224 \\
\hline$x$ & 10302867 \\
\hline$X$ & 10314936 \\
\hline$x$ & 103 \\
\hline
\end{tabular}

\section{2}

2

2

2

2

2

2

2

2

2

2

2

2

2

2

2

2

2

2

2

2

2

2

2

2

2

2

2

2

2

2

2

2

2

2

2

2

2

2

2

2

2

2

2

2

2

2

2

2

2

2

2

2
9

19

15

23

18

6

10

4

15

25

16

14

10

9

10

8

6

13

9

8

9

7

8

9

6

12

5

17

5

12

18

23

11

11

15

13

20

12

6

9

9

8

6

7

9

15

12

5

14

10

5

16

18
4

6

10

6

7

11

5

11

6

8

11

4

5

7

12

6

5

12

12

6

6

12

8

10

7

5

5

20

4

19

17

6

11

16

6

16

4

13

4

4

28

7

6

26

6

4

5

9

5

10

10

13
0

0

0

0

0

0

0

0

0

0

0

0

0

0

0

0

0

0

0

0

0

0

0

0

0

0

0

0

0

0

0

0

0

0

0

0

0

0

0

0

0

0

0

0

0

0

0

0 


\begin{tabular}{|c|c|}
\hline$x$ & 10332087 \\
\hline$x$ & 10332929 \\
\hline$x$ & 10340722 \\
\hline$x$ & 10356035 \\
\hline$x$ & 10369817 \\
\hline$x$ & 10383814 \\
\hline$x$ & 10388269 \\
\hline$x$ & 10388770 \\
\hline$x$ & 10392926 \\
\hline$x$ & 10397161 \\
\hline$x$ & 10426425 \\
\hline$x$ & 10431347 \\
\hline$x$ & 10680623 \\
\hline$x$ & 10716136 \\
\hline$x$ & 10737801 \\
\hline$x$ & 10740595 \\
\hline$x$ & 10746115 \\
\hline$x$ & 10749655 \\
\hline$x$ & 10770635 \\
\hline$x$ & 10888442 \\
\hline$x$ & 10892563 \\
\hline$x$ & 10955764 \\
\hline$x$ & 10982983 \\
\hline$x$ & 10987860 \\
\hline$x$ & 11044494 \\
\hline$x$ & 11137383 \\
\hline$x$ & 11144492 \\
\hline$x$ & 11158856 \\
\hline$x$ & 11199530 \\
\hline$x$ & 11202501 \\
\hline$x$ & 11253688 \\
\hline$x$ & 11304781 \\
\hline$x$ & 11306294 \\
\hline$x$ & 11352230 \\
\hline$x$ & 11362630 \\
\hline$x$ & 11567143 \\
\hline$x$ & 11578704 \\
\hline$x$ & 11619172 \\
\hline$x$ & 11651075 \\
\hline$x$ & 11658407 \\
\hline$x$ & 11701807 \\
\hline$x$ & 11703914 \\
\hline$x$ & 11744343 \\
\hline$x$ & 11769268 \\
\hline$x$ & 11775906 \\
\hline$x$ & 11779923 \\
\hline$x$ & 11795880 \\
\hline$x$ & 11855391 \\
\hline$x$ & 11867221 \\
\hline$x$ & 11876563 \\
\hline$x$ & 11894088 \\
\hline$x$ & 11896933 \\
\hline . & 1191 \\
\hline
\end{tabular}

2

2

2

2

2

2

2

2

2

2

2

2

2

2

2

2

2

2

2

2

2

2

2

2

2

2

2

2

2

2

2

2

2

2

2

2

2

2

2

2

2

2

2

2

2

2

2

2

2

2

2

2

2

$\begin{array}{rr}8 & 12 \\ 9 & \\ 16 & 10 \\ 4 & 12 \\ 16 & \\ 6 & \\ 12 & \\ 4 & 12 \\ 4 & 10 \\ 5 & \end{array}$

12

8

$10 \quad 0$

120

$5 \quad 0$

$8 \quad 0$

120

$16 \quad 0$

$10 \quad 0$

9 O

90

$6 \quad 0$

$8 \quad 0$

$7 \quad 0$

$16 \quad 0$

120

50

$\begin{array}{ll}12 & 0 \\ 10 & 0\end{array}$

$4 \quad 0$

$11 \quad 0$

$8 \quad 0$

$4 \quad 0$

50

$9 \quad 0$

$7 \quad 0$

$15-0$

40

$9 \quad 0$

$8 \quad 0$

$22 \quad 0$

120

190

$7 \quad 0$

$8 \quad 0$

$9 \quad 0$

$7 \quad 0$

$7 \quad 0$

$10 \quad 0$

$5 \quad 0$

170

90

$8 \quad 0$

$8 \quad 0$

$8 \quad 0$

$9 \quad 0$

130

150

40

$7 \quad 0$

$6 \quad 0$

60 


\begin{tabular}{|c|c|}
\hline$x$ & 11938650 \\
\hline$x$ & 11943205 \\
\hline$X$ & 11944820 \\
\hline$x$ & 12026698 \\
\hline$x$ & 12096619 \\
\hline$x$ & 12141978 \\
\hline$x$ & 12207947 \\
\hline$x$ & 12239920 \\
\hline$x$ & 12269545 \\
\hline$x$ & 12313053 \\
\hline$x$ & 12458821 \\
\hline$x$ & 12556147 \\
\hline$x$ & 14603097 \\
\hline$x$ & 14707769 \\
\hline$X$ & 14749593 \\
\hline$x$ & 15048299 \\
\hline$x$ & 15059866 \\
\hline$x$ & 15102053 \\
\hline$x$ & 15114387 \\
\hline$x$ & 15137423 \\
\hline$x$ & 15142288 \\
\hline$x$ & 15162266 \\
\hline$x$ & 15164905 \\
\hline$x$ & 15164922 \\
\hline$x$ & 15167273 \\
\hline$x$ & 15175759 \\
\hline$x$ & 15183258 \\
\hline$x$ & 15185642 \\
\hline$x$ & 15191031 \\
\hline$x$ & 15193675 \\
\hline$x$ & 15212362 \\
\hline$x$ & 15217619 \\
\hline$x$ & 15218834 \\
\hline$x$ & 15227376 \\
\hline$x$ & 15227465 \\
\hline$x$ & 15240498 \\
\hline$x$ & 15249994 \\
\hline$x$ & 15252580 \\
\hline$x$ & 15262518 \\
\hline$x$ & 15266913 \\
\hline$x$ & 15271468 \\
\hline$x$ & 15274752 \\
\hline$x$ & 15275079 \\
\hline$x$ & 15286060 \\
\hline$x$ & 15289847 \\
\hline$x$ & 15297213 \\
\hline$x$ & 15298064 \\
\hline$x$ & 15298998 \\
\hline$x$ & 15319300 \\
\hline$x$ & 15363208 \\
\hline$x$ & 15384676 \\
\hline$x$ & 15385493 \\
\hline$x$ & 15386715 \\
\hline
\end{tabular}

2

\section{2}

2

2

2

2

2

2

2

2

2

2

2

2

2

2

2

2

2

2

2

2

2

2

2

2

2

2

2

2

2

2

2

2

2

2

2

2

2

2

2

2

2

2

2

2

2

2

2

2

2

2

2

$\begin{array}{rr}6 & 1 \\ 4 & \\ 16 & \\ 5 & \\ 4 & 10 \\ 4 & 1 \\ 4 & \\ 9 & \\ 8 & \\ 6 & \\ 9 & \\ 6 & \end{array}$

16

9

6

10

11

9

$5 \quad 0$

$9 \quad 0$

50

$5 \quad 0$

120

$8 \quad 0$

$5 \quad 0$

$10 \quad 0$

50

130

$8 \quad 0$

110

$6 \quad 0$

$6 \quad 0$

$8 \quad 0$

$13 \quad 0$

$6 \quad 0$

$8 \quad 0$

$6 \quad 0$

30

$6 \quad 0$

$7 \quad 0$

$6 \quad 0$

$10 \quad 0$

110

$4 \quad 0$

$9 \quad 0$

$14 \quad 0$

$5 \quad 0$

$15 \quad 0$

$10 \quad 0$

$6 \quad 0$

$13 \quad 0$

$16 \quad 0$

120

$6 \quad 0$

$25 \quad 0$

$7 \quad 0$

$27 \quad 0$

$10 \quad 0$

110

$26 \quad 0$

$8 \quad 0$

$16 \quad 0$

140 


\begin{tabular}{|c|c|}
\hline$x$ & 15394459 \\
\hline$x$ & 15491447 \\
\hline$x$ & 15542905 \\
\hline$x$ & 15998712 \\
\hline$X$ & 15998900 \\
\hline$x$ & 16002074 \\
\hline$x$ & 16002291 \\
\hline$X$ & 16006378 \\
\hline$x$ & 16006430 \\
\hline$x$ & 16018665 \\
\hline$x$ & 16029434 \\
\hline$x$ & 16029737 \\
\hline$x$ & 16033735 \\
\hline$x$ & 16034287 \\
\hline$x$ & 16037960 \\
\hline$x$ & 16038269 \\
\hline$X$ & 16046104 \\
\hline$x$ & 16055780 \\
\hline$x$ & 16068000 \\
\hline$x$ & 16069855 \\
\hline$x$ & 16070101 \\
\hline$x$ & 16128675 \\
\hline$x$ & 16132243 \\
\hline$x$ & 16132391 \\
\hline$x$ & 16132434 \\
\hline$x$ & 16135831 \\
\hline$x$ & 16152971 \\
\hline$x$ & 16154920 \\
\hline$X$ & 16155871 \\
\hline$x$ & 16156065 \\
\hline$x$ & 16162179 \\
\hline$x$ & 16166309 \\
\hline$x$ & 16171818 \\
\hline$x$ & 16172123 \\
\hline$x$ & 16172257 \\
\hline$x$ & 16175381 \\
\hline$x$ & 16176114 \\
\hline$x$ & 16176702 \\
\hline$x$ & 16178011 \\
\hline$x$ & 16179159 \\
\hline$x$ & 16181757 \\
\hline$X$ & 16182549 \\
\hline$x$ & 16186737 \\
\hline$x$ & 16186749 \\
\hline$x$ & 16186872 \\
\hline$x$ & 16192279 \\
\hline$x$ & 16192480 \\
\hline$x$ & 16194422 \\
\hline$x$ & 16200073 \\
\hline$x$ & 16209492 \\
\hline$x$ & 16216333 \\
\hline$x$ & 16218915 \\
\hline$x$ & 16250606 \\
\hline
\end{tabular}

2

\section{2}

2

2

2

2

2

2

2

2

2

2

2

2

2

2

2

2

2

2

2

2

2

2

2

2

2

2

2

2

2

2

2

2

2

2

2

2

2

2

2

2

2

2

2

2

2

2

2

2

2

2

2

23
5
21
5
10
9
16

7

10

11

10

13

5

4

9

7

15

9

22

8

7

7

6

25

9

22

8

18

12

5

8

7

5

6

6

12

12

21

17

20

13

17

22

24

15

5

7

20

15

19

6

14

16
11

4

19

5

21

11

8

8

8

10

70

$5 \quad 0$

$9 \quad 0$

110

250

$\begin{array}{ll}5 & 0 \\ 5 & 0\end{array}$

$15 \quad 0$

50

$15 \quad 0$

$20 \quad 0$

7

7

5

9

10

13

6

7

14

14

7

19

11

5

6

4

10

6

19

4

12

9

4

18$$
0
$$$$
0
$$$$
0
$$$$
0
$$$$
0
$$

0

$$
0
$$$$
0
$$$$
0
$$$$
0
$$$$
0
$$$$
0
$$$$
0
$$

$$
\begin{aligned}
& 0 \\
& 0 \\
& 0 \\
& 0 \\
& 0 \\
& 0 \\
& 0 \\
& 0 \\
& 0 \\
& 0 \\
& 0 \\
& 0
\end{aligned}
$$




\begin{tabular}{|c|c|}
\hline$x$ & 16252195 \\
\hline$x$ & 16254698 \\
\hline$x$ & 16255696 \\
\hline$x$ & 16271594 \\
\hline$x$ & 16273210 \\
\hline$x$ & 16274474 \\
\hline$x$ & 16277263 \\
\hline$x$ & 16277633 \\
\hline$x$ & 16279558 \\
\hline$x$ & 16281667 \\
\hline$x$ & 16281701 \\
\hline$x$ & 16283592 \\
\hline$x$ & 16283607 \\
\hline$x$ & 16284978 \\
\hline$x$ & 16286191 \\
\hline$x$ & 16289601 \\
\hline$x$ & 16291254 \\
\hline$x$ & 16294455 \\
\hline$x$ & 16295584 \\
\hline$x$ & 16300561 \\
\hline$x$ & 16320818 \\
\hline$x$ & 16321083 \\
\hline$x$ & 16321227 \\
\hline$x$ & 16321537 \\
\hline$x$ & 16333870 \\
\hline$x$ & 16350747 \\
\hline$x$ & 16355360 \\
\hline$x$ & 16359591 \\
\hline$x$ & 16359983 \\
\hline$x$ & 16362187 \\
\hline$x$ & 16362524 \\
\hline$x$ & 16368881 \\
\hline$x$ & 16372407 \\
\hline$x$ & 16372414 \\
\hline$x$ & 16375173 \\
\hline$x$ & 16401340 \\
\hline$x$ & 16404418 \\
\hline$x$ & 16408533 \\
\hline$x$ & 16410228 \\
\hline$x$ & 16411828 \\
\hline$x$ & 16416940 \\
\hline$x$ & 16418262 \\
\hline$x$ & 16425056 \\
\hline$x$ & 16427438 \\
\hline$x$ & 16427526 \\
\hline$X$ & 16430791 \\
\hline$x$ & 16433488 \\
\hline$x$ & 16433725 \\
\hline$x$ & 16435014 \\
\hline$x$ & 16435032 \\
\hline$x$ & 16435076 \\
\hline$X$ & 16436554 \\
\hline$x$ & 16448 \\
\hline
\end{tabular}

\begin{tabular}{|c|c|c|c|}
\hline 2 & 7 & 6 & 0 \\
\hline 2 & 14 & 5 & 0 \\
\hline 2 & 4 & 9 & 0 \\
\hline 2 & 9 & 21 & 0 \\
\hline 2 & 5 & 5 & 0 \\
\hline 2 & 19 & 13 & 0 \\
\hline 2 & 6 & 15 & 0 \\
\hline 2 & 9 & 18 & 0 \\
\hline 2 & 9 & 4 & 0 \\
\hline 2 & 19 & 9 & 0 \\
\hline 2 & 17 & 4 & 0 \\
\hline 2 & 23 & 7 & 0 \\
\hline 2 & 26 & 6 & 0 \\
\hline 2 & 10 & 6 & 0 \\
\hline 2 & 18 & 15 & 0 \\
\hline 2 & 12 & 8 & 0 \\
\hline 2 & 16 & 5 & 0 \\
\hline 2 & 21 & 6 & 0 \\
\hline 2 & 4 & 13 & 0 \\
\hline 2 & 10 & 4 & 0 \\
\hline 2 & 10 & 12 & 0 \\
\hline 2 & 22 & 13 & 0 \\
\hline 2 & 5 & 7 & 0 \\
\hline 2 & 24 & 8 & 0 \\
\hline 2 & 25 & 14 & 0 \\
\hline 2 & 15 & 7 & 0 \\
\hline 2 & 11 & 16 & 0 \\
\hline 2 & 6 & 15 & 0 \\
\hline 2 & 14 & 4 & 0 \\
\hline 2 & 5 & 8 & 0 \\
\hline 2 & 10 & 4 & 0 \\
\hline 2 & 15 & 5 & 0 \\
\hline 2 & 8 & 11 & 0 \\
\hline 2 & 11 & 9 & 0 \\
\hline 2 & 15 & 8 & 0 \\
\hline 2 & 14 & 4 & 0 \\
\hline 2 & 7 & 16 & 0 \\
\hline 2 & 5 & 6 & 0 \\
\hline 2 & 4 & 11 & 0 \\
\hline 2 & 14 & 6 & 0 \\
\hline 2 & 16 & 7 & 0 \\
\hline 2 & 15 & 5 & 0 \\
\hline 2 & 10 & 4 & 0 \\
\hline 2 & 10 & 4 & 0 \\
\hline 2 & 30 & 18 & 0 \\
\hline 2 & 12 & 5 & 0 \\
\hline 2 & 26 & 4 & 0 \\
\hline 2 & 12 & 6 & 0 \\
\hline 2 & 5 & 14 & 0 \\
\hline 2 & 5 & 11 & 0 \\
\hline 2 & 8 & 10 & 0 \\
\hline 2 & 5 & 8 & 0 \\
\hline 2 & 9 & 4 & 0 \\
\hline
\end{tabular}




\begin{tabular}{|c|c|}
\hline$x$ & 16451133 \\
\hline$x$ & 16462918 \\
\hline$x$ & 16468122 \\
\hline$x$ & 16485730 \\
\hline$x$ & 16538838 \\
\hline$x$ & 16549141 \\
\hline $\mathrm{X}$ & 16566506 \\
\hline$x$ & 16582796 \\
\hline$x$ & 16604413 \\
\hline$x$ & 16605276 \\
\hline$x$ & 16608976 \\
\hline$X$ & 16622941 \\
\hline$x$ & 16622977 \\
\hline$X$ & 16672209 \\
\hline$x$ & 16726960 \\
\hline$x$ & 16739180 \\
\hline$x$ & 16753076 \\
\hline$x$ & 16792956 \\
\hline$x$ & 16812259 \\
\hline$x$ & 16859496 \\
\hline$x$ & 16889240 \\
\hline$x$ & 16904322 \\
\hline$x$ & 16921852 \\
\hline$x$ & 16926585 \\
\hline$x$ & 16928738 \\
\hline$x$ & 16930783 \\
\hline$x$ & 16934497 \\
\hline$x$ & 16953310 \\
\hline$x$ & 16959502 \\
\hline$x$ & 16965515 \\
\hline$x$ & 16979428 \\
\hline$x$ & 16980644 \\
\hline$x$ & 16991806 \\
\hline$x$ & 17004950 \\
\hline$x$ & 17005004 \\
\hline$x$ & 17016975 \\
\hline$x$ & 17022025 \\
\hline$x$ & 17024729 \\
\hline$x$ & 17042412 \\
\hline$x$ & 17047577 \\
\hline$x$ & 17061564 \\
\hline$x$ & 17062351 \\
\hline$x$ & 17078216 \\
\hline$x$ & 17081163 \\
\hline$x$ & 17082461 \\
\hline$x$ & 17082767 \\
\hline$x$ & 17085423 \\
\hline$x$ & 17088091 \\
\hline$x$ & 17089161 \\
\hline$x$ & 17094652 \\
\hline$x$ & 17094823 \\
\hline$X$ & 17095202 \\
\hline$x$ & 17095568 \\
\hline
\end{tabular}

\begin{tabular}{|c|c|c|}
\hline 2 & 5 & 16 \\
\hline 2 & 5 & 23 \\
\hline 2 & 8 & 8 \\
\hline 2 & 4 & 19 \\
\hline 2 & 11 & 7 \\
\hline 2 & 14 & 4 \\
\hline 2 & 5 & 5 \\
\hline 2 & 9 & 6 \\
\hline 2 & 6 & 13 \\
\hline 2 & 9 & 5 \\
\hline 2 & 12 & 10 \\
\hline 2 & 8 & 5 \\
\hline 2 & 17 & 14 \\
\hline 2 & 5 & 5 \\
\hline 2 & 6 & 8 \\
\hline 2 & 5 & 6 \\
\hline 2 & 6 & 8 \\
\hline 2 & 13 & 7 \\
\hline 2 & 12 & 4 \\
\hline 2 & 5 & 7 \\
\hline 2 & 9 & 4 \\
\hline 2 & 15 & 5 \\
\hline 2 & 9 & 24 \\
\hline 2 & 5 & 5 \\
\hline 2 & 6 & 6 \\
\hline 2 & 12 & 5 \\
\hline 2 & 5 & 6 \\
\hline 2 & 6 & 8 \\
\hline 2 & 7 & 5 \\
\hline 2 & 11 & 5 \\
\hline 2 & 7 & 11 \\
\hline 2 & 13 & 4 \\
\hline 2 & 5 & 21 \\
\hline 2 & 21 & 5 \\
\hline 2 & 32 & 5 \\
\hline 2 & 16 & 16 \\
\hline 2 & 8 & 16 \\
\hline 2 & 19 & 8 \\
\hline 2 & 4 & 20 \\
\hline 2 & 7 & 6 \\
\hline 2 & 7 & 13 \\
\hline 2 & 5 & 5 \\
\hline 2 & 4 & 12 \\
\hline 2 & 4 & 24 \\
\hline 2 & 4 & 12 \\
\hline 2 & 4 & 9 \\
\hline 2 & 17 & 15 \\
\hline 2 & 20 & 26 \\
\hline 2 & 6 & 19 \\
\hline 2 & 14 & 23 \\
\hline 2 & 10 & 21 \\
\hline 2 & 6 & 16 \\
\hline 2 & 7 & 23 \\
\hline
\end{tabular}




\begin{tabular}{|c|c|}
\hline$x$ & 17096042 \\
\hline$x$ & 17096400 \\
\hline$x$ & 17098192 \\
\hline$x$ & 17100250 \\
\hline$x$ & 17100856 \\
\hline$x$ & 17101287 \\
\hline$x$ & 17102819 \\
\hline$x$ & 17109980 \\
\hline$x$ & 17158513 \\
\hline$x$ & 17636400 \\
\hline$x$ & 17636679 \\
\hline$x$ & 17639668 \\
\hline$x$ & 17641428 \\
\hline$x$ & 17641527 \\
\hline$x$ & 17644403 \\
\hline$x$ & 17649368 \\
\hline$x$ & 17652125 \\
\hline$x$ & 17655012 \\
\hline$x$ & 17655648 \\
\hline$x$ & 17664714 \\
\hline$x$ & 17673130 \\
\hline$x$ & 17674802 \\
\hline$x$ & 17674825 \\
\hline$x$ & 17676368 \\
\hline$x$ & 17676434 \\
\hline$x$ & 17676603 \\
\hline$x$ & 17677841 \\
\hline$x$ & 17678091 \\
\hline$x$ & 17682467 \\
\hline$x$ & 17697385 \\
\hline$x$ & 17706408 \\
\hline$x$ & 17706533 \\
\hline$x$ & 17708428 \\
\hline$x$ & 17708528 \\
\hline$x$ & 17710188 \\
\hline$x$ & 17710855 \\
\hline$x$ & 17711563 \\
\hline$x$ & 17713491 \\
\hline$x$ & 17713682 \\
\hline$x$ & 17718102 \\
\hline$x$ & 17721688 \\
\hline$x$ & 17722207 \\
\hline$x$ & 17722416 \\
\hline$x$ & 17724209 \\
\hline$x$ & 17726373 \\
\hline$X$ & 17730959 \\
\hline$x$ & 17731739 \\
\hline$x$ & 17731996 \\
\hline$x$ & 17732231 \\
\hline$x$ & 17732439 \\
\hline$x$ & 17732879 \\
\hline$X$ & 17733107 \\
\hline$x$ & 17733480 \\
\hline
\end{tabular}

\begin{tabular}{|c|c|c|}
\hline \multirow{2}{*}{$\begin{array}{l}2 \\
2\end{array}$} & 23 & 4 \\
\hline & 21 & 10 \\
\hline 2 & 5 & 17 \\
\hline 2 & 30 & 12 \\
\hline 2 & 22 & 25 \\
\hline 2 & 22 & 7 \\
\hline 2 & 19 & 16 \\
\hline 2 & 8 & 17 \\
\hline 2 & 5 & 6 \\
\hline 2 & 12 & 4 \\
\hline 2 & 5 & 6 \\
\hline 2 & 6 & 6 \\
\hline 2 & 10 & 18 \\
\hline 2 & 5 & 8 \\
\hline 2 & 13 & 13 \\
\hline 2 & 6 & 16 \\
\hline 2 & 7 & 16 \\
\hline 2 & 11 & 18 \\
\hline 2 & 6 & 6 \\
\hline 2 & 10 & 14 \\
\hline 2 & 17 & 10 \\
\hline 2 & 10 & 5 \\
\hline 2 & 17 & 7 \\
\hline 2 & 4 & 24 \\
\hline 2 & 6 & 29 \\
\hline 2 & 8 & 7 \\
\hline 2 & 31 & 17 \\
\hline 2 & 9 & 12 \\
\hline 2 & 5 & 15 \\
\hline 2 & 12 & 9 \\
\hline 2 & 11 & 9 \\
\hline 2 & 4 & 14 \\
\hline 2 & 5 & 19 \\
\hline 2 & 12 & 21 \\
\hline 2 & 5 & 17 \\
\hline 2 & 6 & 17 \\
\hline 2 & 16 & 4 \\
\hline 2 & 12 & 4 \\
\hline 2 & 11 & 30 \\
\hline 2 & 17 & 16 \\
\hline 2 & 5 & 8 \\
\hline 2 & 4 & 12 \\
\hline 2 & 13 & 27 \\
\hline 2 & 20 & 5 \\
\hline 2 & 11 & 4 \\
\hline 2 & 11 & 30 \\
\hline 2 & 5 & 12 \\
\hline 2 & 10 & 20 \\
\hline 2 & 17 & 16 \\
\hline 2 & 3 & 31 \\
\hline 2 & 10 & 29 \\
\hline 2 & 7 & 34 \\
\hline 2 & 10 & 2 \\
\hline
\end{tabular}




\begin{tabular}{|c|c|c|c|c|c|}
\hline$x$ & 17733686 & 2 & 7 & 8 & 0 \\
\hline$x$ & 17737524 & 2 & 10 & 21 & 0 \\
\hline$x$ & 17737821 & 2 & 12 & 18 & 0 \\
\hline$x$ & 17738079 & 2 & 8 & 22 & 0 \\
\hline$x$ & 17739190 & 2 & 17 & 17 & 0 \\
\hline$x$ & 17739355 & 2 & 5 & 11 & 0 \\
\hline$x$ & 17755922 & 2 & 13 & 28 & 0 \\
\hline$x$ & 17766984 & 2 & 8 & 9 & 0 \\
\hline$x$ & 17767124 & 2 & 11 & 6 & 0 \\
\hline$x$ & 17772322 & 2 & 6 & 7 & 0 \\
\hline$x$ & 17772371 & 2 & 17 & 11 & 0 \\
\hline$x$ & 17775562 & 2 & 5 & 8 & 0 \\
\hline$x$ & 17775580 & 2 & 5 & 12 & 0 \\
\hline$x$ & 17778512 & 2 & 8 & 27 & 0 \\
\hline$x$ & 17778651 & 2 & 9 & 16 & 0 \\
\hline$x$ & 17779090 & 2 & 4 & 16 & 0 \\
\hline$x$ & 17781894 & 2 & 4 & 15 & 0 \\
\hline$x$ & 17782517 & 2 & 6 & 5 & 0 \\
\hline$x$ & 17800468 & 2 & 5 & 10 & 0 \\
\hline$x$ & 17802202 & 2 & 17 & 6 & 0 \\
\hline$x$ & 17804003 & 2 & 17 & 10 & 0 \\
\hline$x$ & 17804917 & 2 & 28 & 10 & 0 \\
\hline$x$ & 17805346 & 2 & 12 & 6 & 0 \\
\hline$x$ & 17807636 & 2 & 11 & 29 & 0 \\
\hline$x$ & 17808883 & 2 & 9 & 10 & 0 \\
\hline$x$ & 17810353 & 2 & 16 & 11 & 0 \\
\hline$x$ & 17813729 & 2 & 10 & 4 & 0 \\
\hline$x$ & 17814606 & 2 & 4 & 20 & 0 \\
\hline$x$ & 17815257 & 2 & 9 & 19 & 0 \\
\hline$x$ & 17816014 & 2 & 5 & 10 & 0 \\
\hline$x$ & 17816210 & 2 & 9 & 29 & 0 \\
\hline$x$ & 17816295 & 2 & 3 & 34 & 0 \\
\hline$x$ & 17816310 & 2 & 5 & 40 & 0 \\
\hline$x$ & 17826062 & 2 & 11 & 19 & 0 \\
\hline$x$ & 17826604 & 2 & 13 & 30 & 0 \\
\hline$x$ & 17829535 & 2 & 24 & 12 & 0 \\
\hline$x$ & 17831219 & 2 & 16 & 8 & 0 \\
\hline$x$ & 17832304 & 2 & 11 & 6 & 0 \\
\hline$x$ & 17874481 & 2 & 22 & 8 & 0 \\
\hline$x$ & 17876772 & 2 & 8 & 7 & 0 \\
\hline$x$ & 17883037 & 2 & 9 & 4 & 0 \\
\hline$x$ & 17885434 & 2 & 4 & 15 & 0 \\
\hline$x$ & 17886121 & 2 & 11 & 8 & 0 \\
\hline$x$ & 17888697 & 2 & 10 & 8 & 0 \\
\hline$x$ & 17898322 & 2 & 5 & 13 & 0 \\
\hline$x$ & 17898904 & 2 & 4 & 15 & 0 \\
\hline$x$ & 17898989 & 2 & 6 & 9 & 0 \\
\hline$x$ & 17899205 & 2 & 5 & 21 & 0 \\
\hline$x$ & 17900308 & 2 & 20 & 12 & 0 \\
\hline$x$ & 17900860 & 2 & 5 & 12 & 0 \\
\hline$x$ & 17900911 & 2 & 16 & 12 & 0 \\
\hline$x$ & 17903833 & 2 & 22 & 5 & 0 \\
\hline$x$ & 1 & 2 & 14 & 6 & 0 \\
\hline
\end{tabular}




\begin{tabular}{|c|c|}
\hline$x$ & 17918621 \\
\hline $\mathrm{X}$ & 17922656 \\
\hline$x$ & 17923611 \\
\hline$x$ & 17925370 \\
\hline$x$ & 17925814 \\
\hline$x$ & 17926015 \\
\hline$x$ & 17926245 \\
\hline$x$ & 17931066 \\
\hline$x$ & 17934851 \\
\hline$x$ & 17939285 \\
\hline$x$ & 17939779 \\
\hline$x$ & 17941362 \\
\hline$x$ & 17943675 \\
\hline$x$ & 17945957 \\
\hline$x$ & 17947151 \\
\hline$x$ & 17960449 \\
\hline$x$ & 17960968 \\
\hline$x$ & 17961016 \\
\hline$x$ & 17962268 \\
\hline$x$ & 17962458 \\
\hline$x$ & 19098515 \\
\hline$x$ & 19099578 \\
\hline$x$ & 19099892 \\
\hline$x$ & 19101747 \\
\hline$x$ & 19103397 \\
\hline$x$ & 19112930 \\
\hline$x$ & 19113908 \\
\hline$x$ & 19123008 \\
\hline$x$ & 19141251 \\
\hline$x$ & 19152137 \\
\hline$x$ & 19152203 \\
\hline$x$ & 19158485 \\
\hline$x$ & 19159009 \\
\hline$x$ & 19161962 \\
\hline$x$ & 19162084 \\
\hline$x$ & 19166705 \\
\hline$x$ & 19172116 \\
\hline$x$ & 19180738 \\
\hline$x$ & 19185386 \\
\hline$x$ & 19186055 \\
\hline$x$ & 19189843 \\
\hline$x$ & 19190590 \\
\hline$x$ & 19211170 \\
\hline$x$ & 19211754 \\
\hline$x$ & 19213716 \\
\hline$x$ & 19222491 \\
\hline$x$ & 19223662 \\
\hline$x$ & 19224000 \\
\hline$x$ & 19226820 \\
\hline$x$ & 19228767 \\
\hline$x$ & 19231613 \\
\hline$x$ & 19231700 \\
\hline$x$ & 1923450 \\
\hline
\end{tabular}

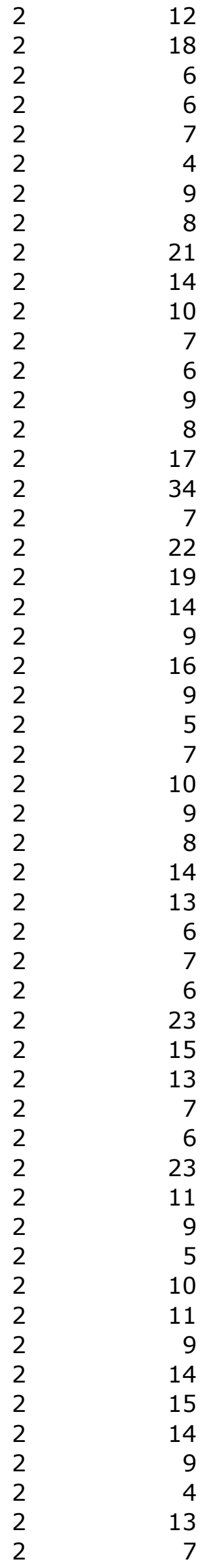

$$
6
$$$$
0
$$$$
8
$$$$
0
$$$$
6
$$$$
5
$$$$
15
$$$$
17
$$$$
9
$$$$
5
$$$$
10
$$$$
8
$$$$
7
$$$$
8
$$$$
14
$$$$
6
$$$$
7
$$$$
9
$$$$
7
$$$$
16
$$$$
6
$$$$
4
$$$$
4
$$$$
16
$$$$
6
$$$$
12
$$$$
4
$$$$
12
$$$$
6
$$$$
8
$$$$
4
$$$$
5
$$$$
8
$$$$
5
$$$$
6
$$$$
9
$$$$
7
$$ 


\begin{tabular}{|c|c|}
\hline$x$ & 19249378 \\
\hline$X$ & 19252806 \\
\hline$x$ & 19302077 \\
\hline$x$ & 19313932 \\
\hline$x$ & 19325792 \\
\hline$x$ & 19325928 \\
\hline$x$ & 19354109 \\
\hline$x$ & 19368654 \\
\hline$x$ & 19370629 \\
\hline$x$ & 19371113 \\
\hline$x$ & 19381694 \\
\hline$x$ & 19397265 \\
\hline$x$ & 19397911 \\
\hline$x$ & 19400540 \\
\hline$x$ & 19419970 \\
\hline$x$ & 19434400 \\
\hline$x$ & 19436103 \\
\hline$x$ & 19436351 \\
\hline$x$ & 19437144 \\
\hline$x$ & 19439240 \\
\hline$x$ & 19440316 \\
\hline$x$ & 19442061 \\
\hline$x$ & 19442836 \\
\hline$x$ & 19444826 \\
\hline$x$ & 19446914 \\
\hline$x$ & 19481114 \\
\hline$x$ & 19481347 \\
\hline$x$ & 19481737 \\
\hline$x$ & 19482442 \\
\hline$x$ & 19487612 \\
\hline$x$ & 19489379 \\
\hline$x$ & 19506965 \\
\hline$x$ & 19507198 \\
\hline$x$ & 19521071 \\
\hline$x$ & 19521507 \\
\hline$x$ & 19524041 \\
\hline$x$ & 19525279 \\
\hline$x$ & 19525366 \\
\hline$x$ & 19527115 \\
\hline$x$ & 19541947 \\
\hline$x$ & 20409822 \\
\hline$x$ & 20410673 \\
\hline$x$ & 20411650 \\
\hline$x$ & 20413082 \\
\hline$x$ & 20415096 \\
\hline$X$ & 20418369 \\
\hline$x$ & 20422073 \\
\hline$x$ & 20422741 \\
\hline$x$ & 20426494 \\
\hline$x$ & 20427526 \\
\hline$x$ & 20440076 \\
\hline$X$ & 20451590 \\
\hline$x$ & 20460126 \\
\hline
\end{tabular}

2

2

2

2

2

2

2

2

2

2

2

2

2

2

2

2

2

2

2

2

2

2

2

2

2

2

2

2

2

2

2

2

2

2

2

2

2

2

2

2

2

2

2

2

2

2

2

2

2

2

2

2

2

11
8
7
9
8
20
7
13

15

12

5

8

16

11

18

4

5
17

17

8

7

5

13

14

10

6

10

17

10

13

33

11

13

8

14

10

10

20

20

11

12

12

7

8

8

12

12

10

10

14

12

14

17

7

5

5

5

4

8

4

9

5

7

\section{7}

5
9

9

6

9

23

8

4

4

8

5

4

14

23

12

3

16

8

8

4

4

7

13

10

5

4

4

7

8

14

5

9

4

5

10

5

14

5

6

6
5
5

5
0

0

0

0

0

0

0

0

0

0

0

0

0

0

0

0

0

0

0

0

0

0

0

0

0

0

0

0

0

0

0

0

0

0

0

0

0

0

0

0

0

0

0

0 


$\begin{array}{ll}X & 20463504 \\ X & 20463909 \\ X & 20470332 \\ X & 20489152 \\ X & 20503402 \\ X & 20573899 \\ X & 20574018 \\ X & 20578070 \\ X & 20583921 \\ X & 20584893 \\ X & 20585127 \\ X & 20634672 \\ X & 20634956 \\ X & 20643155 \\ X & 20644399 \\ X & 20674612 \\ X & 20686174 \\ X & 20703252 \\ X & 20715600 \\ X & 20722902 \\ X & 20723988 \\ X & 20741783 \\ X & 20743149 \\ X & 20746322 \\ X & 20748655 \\ X & 20750151 \\ X & 20753738 \\ X & 20757373 \\ X & 20796458 \\ X & 20819537 \\ X & 20847281 \\ X & 20854993 \\ X & 20873814 \\ X & 20898829 \\ X & 20919300 \\ X & 20920968 \\ X & 20938290 \\ X & 20951061 \\ X & 20971255 \\ X & 20973497 \\ X & 2110602 \\ X & \end{array}$

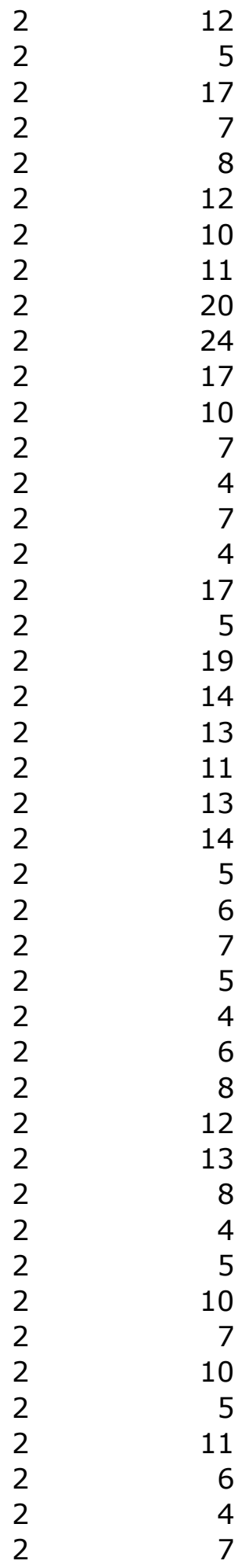

\section{4}

6

9

15

8

7

8

6

8

5

9

$$
5
$$$$
7
$$

16

10

10

12

9

10

10

8

8

5

8

12

8

7

5

15

5

5

7

5

13

21

13

14

25

16

5

15

22

14

9
0

0

0

0

0

0

0

0

0

0

0

0

0

0

0

0

0

0

0

0

0

0

0

0

0

0

0

0

0

0

0

0

0

0

0

0

0

0

0

0

0

0

0 


\section{Supplemental Table 2: Differential non-synonymous SNPs}

\begin{tabular}{|c|c|c|}
\hline Chr & position & $\begin{array}{l}\text { Gene } \\
\text { Affected }\end{array}$ \\
\hline $2 \mathrm{~L}$ & 2204937 & 7 CG31668 \\
\hline $2 \mathrm{~L}$ & 2421314 & 4 CG9886 \\
\hline $2 \mathrm{~L}$ & 3472150 & CG31776 \\
\hline $2 \mathrm{~L}$ & 8765945 & 5 CG9463 \\
\hline $2 \mathrm{~L}$ & 10136914 & C CG13136 \\
\hline $2 \mathrm{~L}$ & 10151359 & CG4839 \\
\hline $2 \mathrm{~L}$ & 10152808 & C CG4839 \\
\hline $2 \mathrm{~L}$ & 10199877 & 7 Ripalpha \\
\hline $2 \mathrm{R}$ & 3944833 & 3 CG12769 \\
\hline $2 \mathrm{R}$ & 3975602 & 2 sut2 \\
\hline $2 \mathrm{R}$ & 4010646 & 5 CG2158 \\
\hline $2 \mathrm{R}$ & 4099635 & 5 CG33087 \\
\hline $2 \mathrm{R}$ & 4142597 & 7 CG14760 \\
\hline $2 \mathrm{R}$ & 4235896 & 5 CG14755 \\
\hline $2 \mathrm{R}$ & 4246799 & CG11635 \\
\hline $2 \mathrm{R}$ & 4246951 & LG11635 \\
\hline $2 \mathrm{R}$ & 4248042 & spaw \\
\hline $2 \mathrm{R}$ & 4249339 & 9 hubl \\
\hline $2 \mathrm{R}$ & 4251702 & swif \\
\hline $2 \mathrm{R}$ & 4278507 & $7 \mathrm{pdm} 3$ \\
\hline $2 \mathrm{R}$ & 4291588 & CG2121 \\
\hline $2 \mathrm{R}$ & 4291670 & CG2121 \\
\hline $2 \mathrm{R}$ & 4358664 & 4 CG11669 \\
\hline $2 \mathrm{R}$ & 4358944 & CG11669 \\
\hline $2 \mathrm{R}$ & 4359074 & 4 CG11669 \\
\hline $2 \mathrm{R}$ & 4458819 & С Сур6а13 \\
\hline $2 \mathrm{R}$ & 4459026 & 5 Сур6а13 \\
\hline $2 \mathrm{R}$ & 4468681 & 1 CG42326 \\
\hline $2 \mathrm{R}$ & 4471074 & 4 CG42326 \\
\hline $2 \mathrm{R}$ & 4498388 & $3 \mathrm{rgr}$ \\
\hline $2 \mathrm{R}$ & 4501379 & 7 CG8642 \\
\hline $2 \mathrm{R}$ & 4504543 & 3 Cirl \\
\hline $2 \mathrm{R}$ & 4513500 & Vps25 \\
\hline $2 \mathrm{R}$ & 4514607 & 7 beta3GalTII \\
\hline $2 \mathrm{R}$ & 4514974 & 4 beta3GalTII \\
\hline $2 \mathrm{R}$ & 4516141 & CG14749 \\
\hline $2 \mathrm{R}$ & 4516450 & CG14749 \\
\hline $2 \mathrm{R}$ & 4516643 & CG14749 \\
\hline $2 \mathrm{R}$ & 4549769 & 9 ptc \\
\hline $2 \mathrm{R}$ & 4564849 & CG30354 \\
\hline $2 \mathrm{R}$ & 4567125 & 5 CG30355 \\
\hline $2 \mathrm{R}$ & 4586026 & 5 CG8738 \\
\hline $2 \mathrm{R}$ & 4589005 & 5 CG8584 \\
\hline $2 \mathrm{R}$ & 4595697 & 7 CG14744 \\
\hline $2 \mathrm{R}$ & 4604541 & 1 PGRP-SC2 \\
\hline $2 \mathrm{R}$ & 4680279 & CG8746 \\
\hline $2 \mathrm{R}$ & 4719279 & 9 sns \\
\hline $2 \mathrm{R}$ & 4799432 & $2 \mathrm{Pgi}$ \\
\hline $2 R$ & 4814797 & 7 CG8235 \\
\hline
\end{tabular}

nt position Reference in CDS

725 GGA 419 GCC

1718 CGT

2485 CTG 442 GAT

805 CCG

1213 AGC

549 CAA

4 GCC

482 AGT

$4 \mathrm{GCT}$

2023 GCC

413 AAG

853 TAA

688 GCG

840 AAC

386 CCA

266 GCT

149 AGC

1712 GGC

173 GCC

91 ACT

491 AGG

211 GAG

81 GAA

784 AAC

991 CGC

1177 CCA

56 GCA

2566 AAG

38 GAC

374 TCG

418 TAC

483 AAT

116 AGA

595 GGC

904 GAG

1097 ACT

2105 CAG

10 AAT

209 GGA

1376 TाT

129 TTG

857 TTA

70 ATC

25 TCA

2414 AAG

985 GTT

752 AGT differen

ce

$\begin{array}{ll} & \text { p-score } \\ 2 & \\ 2 & \\ 2 & \\ 2 & \\ 2 & \\ 2 & \\ 2 & \\ 2 & \\ 2 & \end{array}$

0 
$2 \mathrm{R}$

$2 R$

$2 R$

$2 R$

$2 \mathrm{R}$

$2 \mathrm{R}$

$2 \mathrm{R}$

$2 R$

$2 \mathrm{R}$

$2 \mathrm{R}$

$2 \mathrm{R}$

$2 R$

$2 R$

$2 \mathrm{R}$

$2 R$

$2 \mathrm{R}$

$2 \mathrm{R}$

$2 R$

$2 \mathrm{R}$

$2 R$

$2 \mathrm{R}$

$2 \mathrm{R}$

$2 \mathrm{R}$

$2 \mathrm{R}$

$2 \mathrm{R}$

$2 \mathrm{R}$

$2 \mathrm{R}$

$2 \mathrm{R}$

$3 \mathrm{~L}$

$3 \mathrm{~L}$

$3 \mathrm{~L}$

$3 \mathrm{~L}$

$3 \mathrm{~L}$

$3 \mathrm{~L}$

$3 \mathrm{~L}$

$3 \mathrm{~L}$

$3 \mathrm{~L}$

$3 \mathrm{~L}$

$3 \mathrm{~L}$

$3 \mathrm{~L}$

$3 \mathrm{~L}$

$3 \mathrm{~L}$

$3 \mathrm{~L}$

$3 \mathrm{~L}$

$3 \mathrm{~L}$

$3 \mathrm{~L}$

$3 \mathrm{~L}$

$3 \mathrm{~L}$

$3 \mathrm{~L}$

$3 \mathrm{~L}$

$3 \mathrm{~L}$

$3 L$

3L
4821067 CG13749

4823787 CG13745

4837720 CG8229

4949324 CG8197

4987386 CG8777

4990312 CG8777

5015154 CG18659

5022116 Hydr1

5051742 CG8046

5118618 hig

5127659 Сур4p1

5131086 Сур4p3

5143402 Pkn

5148541 CG34141

5195197 Myd88

5195251 Myd88

5207041 CG13739

5233297 Or45a

5233621 Or45a

$5279323 \mathrm{I}(2) 03659$

5302638 wun2

5304732 wun2

5310214 CG13955

5310983 CG13955

5341512 CG33758

7126386 CG13216

7140393 Cpr47Ea

7217607 sha

1647061 CG17249

1657154 CG7971

1662211 CG7971

1664682 CG7971

1701847 CG13931

1710160 CG13932

1717029 drpr

1717786 drpr

$1718037 \mathrm{drpr}$

1724921 CG12035

1775879 CG12018

$1790210 \mathrm{dlt}$

1803869 CG12022

1863777 HBS1

1936174 CG5687

1943883 mu2

1944019 mu2

1944635 mu2

2021527 CG42357

2021543 CG42357

2022424 CG42356

2056108 sls

2106318 sls

2153903 CG15822

2255756 CG1275

\begin{tabular}{|c|c|}
\hline 65 CGG & 2 \\
\hline 2840 AAG & 2 \\
\hline 373 CAC & 2 \\
\hline 42 GAA & 2 \\
\hline 989 ТАТ & 2 \\
\hline 3449 GAT & 2 \\
\hline 1027 AGC & 2 \\
\hline 1136 CTT & 2 \\
\hline 532 GCC & 2 \\
\hline 1652 ACA & 2 \\
\hline 73 CAC & 2 \\
\hline 914 GGC & 2 \\
\hline 319 GAG & 2 \\
\hline 1732 CCC & 2 \\
\hline 103 CCC & 2 \\
\hline 49 CAC & 2 \\
\hline 633 CAC & 2 \\
\hline 371 AGG & 2 \\
\hline 695 GGG & 2 \\
\hline 736 AGG & 2 \\
\hline 73 GGT & 2 \\
\hline 750 GAG & 2 \\
\hline 769 ATC & 2 \\
\hline 145 GCC & 2 \\
\hline 49 GTT & 2 \\
\hline 83 GCG & 2 \\
\hline 4 TCG & 2 \\
\hline 251 AGC & 2 \\
\hline 340 GCG & 2 \\
\hline 2291 CTA & 2 \\
\hline 512 ACC & 2 \\
\hline 89 AAG & 2 \\
\hline 67 GTG & 2 \\
\hline 121 TCA & 2 \\
\hline 2666 GGC & 2 \\
\hline 1979 TAC & 2 \\
\hline 1795 GCC & 2 \\
\hline 361 GCC & 2 \\
\hline 199 GAC & 2 \\
\hline 3206 AAA & 2 \\
\hline 49 ССТ & 2 \\
\hline 1269 GAT & 2 \\
\hline 107 ТCT & 2 \\
\hline 3169 GGC & 2 \\
\hline 3033 GAG & 2 \\
\hline 2417 GCC & 2 \\
\hline 432 CAT & 2 \\
\hline 416 TAC & 2 \\
\hline 968 CAG & 2 \\
\hline 40696 CAA & 2 \\
\hline 589 TAC & 2 \\
\hline 3763 GCT & 2 \\
\hline 57 CAG & 2 \\
\hline
\end{tabular}


$3 \mathrm{~L}$

$3 L$

$3 L$

3L

3L

$3 L$

3L

$3 \mathrm{~L}$

3L

3L

3L

3L

$3 \mathrm{~L}$

3L

3L

$3 \mathrm{~L}$

$3 \mathrm{~L}$

$3 \mathrm{~L}$

$3 \mathrm{~L}$

$3 \mathrm{~L}$

$3 \mathrm{~L}$

$3 \mathrm{~L}$

$3 \mathrm{~L}$

$3 \mathrm{~L}$

$3 \mathrm{~L}$

$3 \mathrm{~L}$

$3 \mathrm{~L}$

$3 \mathrm{~L}$

$3 \mathrm{~L}$

$3 \mathrm{~L}$

$3 \mathrm{~L}$

$3 \mathrm{~L}$

$3 \mathrm{~L}$

$3 \mathrm{~L}$

$3 \mathrm{~L}$

$3 \mathrm{~L}$

3R

$3 R$

$3 R$

$3 R$

$3 R$

$3 R$

$3 R$

$3 R$

$3 R$

$3 R$

$3 R$

$3 R$

$3 R$

$3 R$

$3 R$

$3 R$

$3 R$
2259387 oxt

2374849 CG9004

2439782 CG32306

2463342 CG32306

2466023 CG33231

2598056 CG12182

2773225 CG12093

2789467 CG2083

2790396 CG2083

2794571 CG2083

2915999 CG9970

2982781 CG2107

3008755 FR

3190168 CG14958

3296715 CG12009

3335646 Drs-I

3930839 Eip63F-1

3944294 CG14982

$4020755 \mathrm{fd} 64 \mathrm{~A}$

4035868 Gr64f

4113153 Ack

4143576 CG32260

4289491 Hexo1

4391130 CG7447

5496689 CG34342

$5511664 \mathrm{Lkr}$

6066508 CG6602

6619629 Glu-RI

8830298 CG32026

20204278 CG7017

20212064 CG6933

20339421 CG5585

20467709 CG5976

20710240 CG34260

21928511 CG7442

22273050 CG11489

30845 CG14636

367961 Mur82C

368261 Mur82C

456051 5-HT2

529962 CG31531

999585 cno

1046590 CG1116

1199299 hd

$1295900 \mathrm{mRpL} 44$

1409251 CG2926

1409441 CG2926

$1583583 \mathrm{sec} 8$

1853984 CG1075

2162631 Osi19

2172617 CG18048

2183992 Pak

2195528 CG10286
2573 GCG

1001 ATG

1936 CCA

5633 GAG

476 GAC

1070 ATG

584 TTG

5887 GGA

4958 AGC

1124 CCG

590 GTC

1598 GGA

10 ACA

70 GTA

731 AGT

143 TGC

89 AAA

700 TCT

674 AGC

70 TTC

2588 CAG

391 CCA

323 GTT

640 TCT

1706 GCG

14 TTA

1265 CAG

236 GTC

693 GAA

585 TTG

707 GTA

1304 TAT

83 CTA

850 GCC

360 GAC

1892 TCA

643 ATG

704 CCA

1004 ACA

2114 CTT

3701 AGT

5589 AAG

660 ATG

805 GCC

349 GAT

3727 TTT

3537 AGC

627 GAG

433 CGC

770 GGG

703 ATC

1393 АTT

790 GAT
2

2

2

2

2

2

2

2

2

2

2

2

2

2

2

2

2

2

2

2

2

2

2

2

2

2

2

2

2

2

2

2

2

2

2

2

2

2

2

2

2

2

2

2

2

2

2

2

2

2

2

2

2
0

0

0

0

0

0

0

0

0

0

0

0

0

0

0

0

0
0

0

0

0

0

0

0

0

0

0

0

0

0

0

0

0

0

0

0

0

0

0

0

0

0

0

0

0

0

0

0

0

0

0

0

0 
$3 R$

$3 R$

3R

$3 R$

$3 R$

$3 R$

$3 R$

$3 R$

$3 R$

$3 R$

$3 R$

$3 R$

$3 R$

$3 R$

$3 R$

$3 R$

$3 R$

$3 R$

$3 R$

$3 R$

$3 R$

$3 R$

$3 R$

$3 R$

$3 R$

$3 R$

$3 R$

$3 R$

$3 R$

$3 R$

$3 R$

$3 R$

$3 R$

$3 R$

$3 R$

$X$

$x$

$x$

$x$

$x$

$x$

$x$

$x$

$x$

$x$

$x$

$x$

$x$

$x$

$x$

$x$

$x$

$x$
2195591 CG10286

2200354 CG42564

2891049 CG1105

3078885 Gld

3097821 CG10032

3247171 wa-cup

3327872 CG1287

3800401 CD98hc

3911042 CG7918

4067941 CG9636

5301391 CG16779

5408269 CG33654

5458206 SpdS

$5474860 \mathrm{Dh}$

5838019 Mical

6152798 Bruce

6622501 CG14692

6628968 CG14692

6720691 CG4674

6990776 Ugt86Dg

7278803 PGRP-LB

$7360421 \mathrm{dpr} 4$

7579202 Lk6

7802091 CG10005

7874179 CG14739

7990042 Cpn

8061395 Cyp313a2

8190694 GstD10

8247252 Scgbeta

8834623 CG8031

9206033 CG8870

$10130589 \mathrm{su}(\mathrm{Hw})$

$17201269 \bmod (\operatorname{mdg} 4)$

20691742 Ela

20747095 CG11168

$142650 \mathrm{G} 9 \mathrm{a}$

153547 CG13377

202395 CG12470

$524614 \mathrm{I}(1) 1 \mathrm{Bi}$

524854 I(1)1Bi

638205 sdk

821204 CG3711

823788 CG3708

922020 CG3021

1094788 CG14625

1104881 CG11382

1116694 CG3638

1189782 DAAM

$1218809 \mathrm{fs}(1) \mathrm{N}$

$1221603 \mathrm{fs}(1) \mathrm{N}$

1237093 CG11412

1328825 futsch

1333940 futsch

\begin{tabular}{|c|c|}
\hline 853 GCC & 2 \\
\hline 154 CTA & 2 \\
\hline 665 AAG & 2 \\
\hline 1451 CAG & 2 \\
\hline 508 CTG & 2 \\
\hline 479 CAT & 2 \\
\hline 94 GCG & 2 \\
\hline 1638 GAT & 2 \\
\hline 2648 GGG & 2 \\
\hline 984 CAT & 2 \\
\hline 115 GCA & 2 \\
\hline 475 GCG & 2 \\
\hline 242 CCC & 2 \\
\hline 486 GAA & 2 \\
\hline 6502 ATC & 2 \\
\hline 2932 GCA & 2 \\
\hline 5860 AAG & 2 \\
\hline 664 GTG & 2 \\
\hline 1355 GCC & 2 \\
\hline 833 GGC & 2 \\
\hline 625 GTG & 2 \\
\hline 773 ACC & 2 \\
\hline 2921 AAG & 2 \\
\hline 227 ATG & 2 \\
\hline 516 GAG & 2 \\
\hline 1231 TTA & 2 \\
\hline 952 GAA & 2 \\
\hline 574 ССС & 2 \\
\hline 814 ATT & 2 \\
\hline 671 AAT & 2 \\
\hline 116 ССС & 2 \\
\hline 2767 TCA & 2 \\
\hline 694 ТСТ & 2 \\
\hline 484 ТСТ & 2 \\
\hline 1954 GAG & 2 \\
\hline 983 ССТ & 2 \\
\hline 148 ATA & 2 \\
\hline 155 CGG & 2 \\
\hline 633 CAG & 2 \\
\hline 815 गा & 2 \\
\hline 3904 AAA & 2 \\
\hline 277 GCC & 2 \\
\hline 269 CGG & 2 \\
\hline 1007 СТT & 2 \\
\hline 185 GCT & 2 \\
\hline 752 AAC & 2 \\
\hline 1656 CAA & 2 \\
\hline 212 GGA & 2 \\
\hline 2145 CAG & 2 \\
\hline 4652 GGA & 2 \\
\hline 512 AGT & 2 \\
\hline 2395 ACT & 2 \\
\hline 7510 ТСТ & 2 \\
\hline
\end{tabular}




\begin{tabular}{|c|c|}
\hline$x$ & 1335090 futsch \\
\hline$x$ & 1335212 futsch \\
\hline$x$ & 1335668 futsch \\
\hline$x$ & 1338741 futsch \\
\hline$x$ & 1339745 futsch \\
\hline$x$ & 1339842 futsch \\
\hline$x$ & 1340403 futsch \\
\hline$x$ & 1340492 futsch \\
\hline$x$ & 1340961 futsch \\
\hline$x$ & 1340982 futsch \\
\hline$x$ & 1346885 Gr2a \\
\hline$x$ & $1347421 \mathrm{Gr} 2 \mathrm{a}$ \\
\hline$x$ & 1351392 CG14786 \\
\hline$x$ & 1351840 CG14786 \\
\hline$x$ & 1353689 CG14786 \\
\hline$x$ & 1355476 CG14787 \\
\hline$x$ & 1371928 mei-38 \\
\hline$x$ & $1563960 \mathrm{hfw}$ \\
\hline$x$ & 1571189 CG32809 \\
\hline$x$ & 1715591 CG14811 \\
\hline$x$ & 1770935 CG14817 \\
\hline$x$ & $1783154 \mathrm{trr}$ \\
\hline$x$ & 1813236 mip 130 \\
\hline$x$ & 1818569 CG3573 \\
\hline$x$ & $1892668 \mathrm{Hr} 4$ \\
\hline$x$ & 1902204 CG4406 \\
\hline$x$ & 1908658 east \\
\hline$x$ & 1913190 east \\
\hline$x$ & 1950522 CG4290 \\
\hline$x$ & 1958445 CG4281 \\
\hline$x$ & 1968192 CG4061 \\
\hline$x$ & 1968446 CG4061 \\
\hline$x$ & 1972008 CG4025 \\
\hline$x$ & 1975347 Unc-76 \\
\hline$x$ & 2076057 pn \\
\hline$x$ & 2086871 CG14053 \\
\hline$x$ & 2086921 CG14053 \\
\hline$x$ & 2110740 Vinc \\
\hline$x$ & 2122748 pсx \\
\hline$x$ & 2148093 CG3091 \\
\hline$x$ & 2150964 CG3078 \\
\hline$x$ & 2152227 CG3078 \\
\hline$x$ & 2219071 CG14050 \\
\hline$x$ & 2383061 trol \\
\hline$x$ & 3857112 Iva \\
\hline$x$ & 3858259 Iva \\
\hline$x$ & 3858500 Iva \\
\hline$x$ & 4425385 CG3556 \\
\hline$x$ & 4425553 CG3556 \\
\hline$x$ & 4522861 CG12184 \\
\hline$X$ & 4526638 CG12179 \\
\hline$x$ & 4532126 pon \\
\hline$x$ & $4547002 \mathrm{C}$ \\
\hline
\end{tabular}

\begin{tabular}{|c|c|}
\hline 8660 TGT & 2 \\
\hline 8782 ACG & 2 \\
\hline 9238 ATG & 2 \\
\hline 12311 GTG & 2 \\
\hline 13315 ACC & 2 \\
\hline 13412 TCC & 2 \\
\hline 13973 ACC & 2 \\
\hline 14062 TTC & 2 \\
\hline 14531 CAG & 2 \\
\hline 14552 GGC & 2 \\
\hline 150 ATA & 2 \\
\hline 686 GAG & 2 \\
\hline 3139 СТT & 2 \\
\hline 2691 TTG & 2 \\
\hline 903 CAG & 2 \\
\hline 203 ATG & 2 \\
\hline 617 AGC & 2 \\
\hline 8 GCG & 2 \\
\hline 2499 CAC & 2 \\
\hline 593 ACA & 2 \\
\hline 181 ТTC & 2 \\
\hline 592 ТСТ & 2 \\
\hline 2230 AGC & 2 \\
\hline 304 AGC & 2 \\
\hline 1049 GTG & 2 \\
\hline 935 CGG & 2 \\
\hline 104 GAC & 2 \\
\hline 3824 GCT & 2 \\
\hline 3720 CAC & 2 \\
\hline 469 TCA & 2 \\
\hline 449 GTT & 2 \\
\hline 703 CAT & 2 \\
\hline 643 GTC & 2 \\
\hline 209 GCG & 2 \\
\hline 657 AGC & 2 \\
\hline 133 CAG & 2 \\
\hline 83 GTG & 2 \\
\hline 1446 CAG & 2 \\
\hline 2808 TTG & 2 \\
\hline 28 TCC & 2 \\
\hline 1099 TTG & 2 \\
\hline 220 TCA & 2 \\
\hline 568 GCT & 2 \\
\hline 6493 АТT & 2 \\
\hline 1481 CTA & 2 \\
\hline 334 CGC & 2 \\
\hline 157 AAA & 2 \\
\hline $43 \mathrm{CTT}$ & 2 \\
\hline $211 \mathrm{CTT}$ & 2 \\
\hline 2003 GAG & 2 \\
\hline 3172 TAC & 2 \\
\hline 1447 TCC & 2 \\
\hline 1229 ACA & 2 \\
\hline
\end{tabular}


$X$

$X$

$X$

$X$

$\mathrm{X}$

$X$

$\mathrm{X}$

$x$

$x$

$x$

$x$

$x$

$x$

$x$

$x$

$x$

$X$

$x$

$X$

$x$

$X$

$X$

$x$

$x$

$X$

$x$

$x$

$x$

$x$

$x$

$x$

$x$

$x$

$x$

$x$

$x$

$X$

$X$

$X$

$X$

$X$

$x$

$x$

$x$

$X$

$X$

$X$

$X$

$X$

$X$

$X$

$x$

$X$
4548151 CG15473

4575447 Pp2C1

4811711 CG4041

5119409 rg

5130418 rg

5208831 CG4198

5213689 Rnp4F

5222015 CG3309

5225274 XRCC1

5388885 CG3239

5518663 I(1)G0045

5518864 I(1)G0045

5522954 CG42492

5563355 CG4119

5574799 Tre

5576069 Tre

5579952 CG3160

5583221 CG3160

5661340 CG3097

5670273 CG42264

5671757 CG3108

5679990 CG4064

5709316 CG15766

5731951 CG15765

5732628 CG15765

5732650 CG15765

5732695 CG15765

5739627 CG12729

5766338 CG3033

5810495 CG3011

5833987 CG3726

5834108 CG3726

5851452 CG6067

5852573 fs(1)M3

5854417 fs(1)M3

5854445 fs(1)M3

5854809 fs(1)M3

$5856938 \mathrm{fs}(1) \mathrm{M} 3$

5856975 fs(1)M3

5878369 Grip

5879133 Grip

6582487 CG3184

6606813 CG14441

6655602 CG14438

6657921 CG14438

6658333 CG14438

6664242 CG14438

6716548 CG4542

6720115 Smg1

6722752 Smg1

6722847 Smg1

6725519 CG12796

6739302 CG4557
157 TTC

3502 GCT

446 GCC

653 ACC

4121 ACT

1300 GTG

2377 AAT

1229 CTC

1079 TCG

809 AAT

1840 TCT

2041 AAG

2939 GAG

2278 AAC

40 GTT

997 CCC

2936 CGA

$151 \mathrm{GTT}$

388 GGT

340 AAG

3082 CGT

1376 TCC

484 GTA

1340 ACA

2017 ACA

2039 GAA

2084 TCG

242 TAT

892 TTC

8 CGG

1100 CTA

1221 GAG

374 GCG

5267 CCA

3423 AAT

3395 TGT

3031 GTC

1124 CAC

1087 GCG

1307 ACT

2071 ACA

287 CCC

679 GAT

1417 ताT

3736 CAA

4148 GCT

9854 TCT

1238 ATG

1306 GTG

3875 GCT

3970 CTG

1106 GCC

595 GTC
2

2

2

2

2

2

2

2

2

2

2

2

2

2

2

2

2

2

2

2

2

2

2

2

2

2

2

2

2

2

2

2

2

2

2

2

2

2

2

2

2

2

2

2

2

2

2

2

2

2

2

2

2
0

0

0

0

0

0

0

0

0

0

0

0

0

0

0

0

0

0

0

0

0

0

0

0

0

0

0

0

0

0

0

0

0

0

0

0

0

0

0

0

0

0

0

0

0

0

0

0

0

0

0

0 
$X$

$X$

$X$

$X$

$\mathrm{X}$

$X$

$\mathrm{X}$

$\mathrm{X}$

$x$

$x$

$x$

$x$

$x$

$x$

$x$

$x$

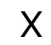

$x$

$x$

$X$

$X$

$x$

$x$

$x$

$X$

$x$

$x$

$x$

$x$

$x$

$X$

$x$

$x$

$x$

$x$

$x$

$X$

$X$

$X$

$X$

$X$

$X$

$x$

$x$

$x$

$x$

$x$

$X$

$X$

$X$

$X$

$x$

$X$
6770464 CG33691

6771120 CG33691

6865776 CG4586

6905903 CG32736

7001469 fz4

7404404 CG32719

7405239 CG32719

7624591 CG12155

7626150 Pdp

7762434 dec-1

7769889 Ir7c

7770020 Ir7c

7771042 Ir7d

7771187 Ir7d

7771678 Ir7d

7775247 Ir7e

7777767 Ir7f

7807628 CG1514

$7812726 \mathrm{hdm}$

7812923 hdm

$7813471 \mathrm{hdm}$

7929981 CG2233

7930582 CG2233

7931406 CG2233

7970367 CG1571

8499315 Caf1-180

10058842 CG32691

10082372 CG15311

10083535 CG15311

15218834 Top1

15240498 CG6227

16295584 CG9981

16333870 CG9947

16979428 CG8945

17096400 CG8918

17100856 CG32564

17102819 CG32563

17644403 CG42270

17713491 CG12986

17755922 CG6769

17782517 CG6788

17939285 CG15373

17939779 CG15373

17941362 CG15373

19098515 Ulp1

19099578 Ulp1

19099892 Ulp1

19101747 Ulp1

19368654 CG32533

19371113 CG32533

$19381694 \mathrm{pcm}$

19400540 Pfrx

19489379 CG14205
$71 \mathrm{TT}$

727 GGT

1618 GCT

207 CAT

2063 CAG

2183 GAA

1348 CAG

1081 GCC

1313 GCG

4419 AAG

935 ACA

1066 GTG

1739 CGA

1594 ACG

1103 TGG

466 ATC

293 CGT

460 CTG

1285 GAG

1088 CAA

694 CAA

1241 ATA

700 GAG

113 AGC

181 GTC

907 ATG

235 CAT

489 ATC

1652 CAA

2020 CGC

661 CTG

1655 ACA

852 AAC

2218 GGC

152 AAA

349 GTC

290 CTG

4037 CAC

158 CCA

568 GAC

296 GCC

713 CCC

960 CAG

2309 GGC

3142 GTG

2215 CAT

1901 GAT

46 TAC

2981 ACT

1934 GTG

3479 CCG

131 CCC

2098 GCA
2

2

2

2

2

2

2

2

2

2

2

2

2

2

2

2

2

2

2

2

2

2

2

2

2

2

2

2

2

2

2

2

2

2

2

2

2

2

2

2

2

2

2

2

2

2

2

2

2

2

2

2

2
0

0

0

0

0

0

0

0

0

0

0

0

0

0

0

0

0

0

0

0

0

0

0

0

0

0

0

0

0

0

0

0

0

0

0

0

0

0

0

0

0

0

0

0

0

0

0

0

0

0

0

0 
bioRxiv preprint doi: https://doi.org/10.1101/119719; this version posted March 24, 2017. The copyright holder for this preprint (which was

not certified by peer review) is the author/funder, who has granted bioRxiv a license to display the preprint in perpetuity. It is made available under aCC-BY-NC-ND 4.0 International license. 